\title{
Music and philosophical naturalism
}

by

Anton Killin

\begin{abstract}
A thesis
submitted to the Victoria University of Wellington in fulfilment of the requirements for the degree of Doctor of Philosophy
\end{abstract}

Victoria University of Wellington

2016 


\section{Abstract}

The philosophical and scientific explication of music is a cutting-edge field in contemporary academia. This thesis develops a naturalistic framework for theorising about music. The following novel philosophical positions are motivated and defended: a polysemy analysis of "sound", conceptual pluralism about music, a pluralistic framework for approaching the science of music, and a fictionalist account of Western musical artworks. The adaptation/ by-product framework for couching discussion about the evolution of music is critiqued. A novel, co-evolutionary, niche construction model of the foundations of musicality and the origins, expansion and stabilisation of music is developed, couched in the general context of hominin evolution and prehistory. Conceptual and methodological reflection accompanies the evolutionary scenario developed.

\section{Keywords}

Music; Philosophical naturalism; Philosophy of music; Sound; Polysemy; Conceptual pluralism; Musical works; Ontology of music; Fictionalism; Evolution of music; Musicality; Philosophy of science; Philosophy of biology; Philosophy of nature; Hominin evolution; Music archaeology; Ethnomusicology. 


\section{Acknowledgments}

A thesis is not written in a vacuum. I have had an enormous amount of assistance and support along the way from many amazing people. But a few people stand out.

I am hugely indebted to my two supervisors, Stuart Brock and Kim Sterelny.

Stu, Kimbo: thanks for everything!

Simon Keller provided valuable feedback on draft material behind chapter 2.

I have greatly benefitted from my collaborative research with Adrian Currie on conceptual pluralism about music. The fruits of this collaborative work appears here (chapters 3 and 4) in a rewritten and extended form. (So Adrian's not to blame for my articulation and extension of that research!)

I have also greatly benefitted from many long discussions about hominin evolution with Kim Shaw-Williams, impacting my views especially in chapter 7.

My partner Johanna Guest has been an inspiration and an incredible influence, both personally and philosophically. Jo: your love and support means the world to me.

Mum, Dad, and my sister Crystal have never failed to take an interest in my studies, and have never failed to encourage or assist me. Thankyou!

For financial/administrative assistance I thank Barry Lewis and the VUW Scholarships Office.

Finally, cheers to all the VUW and ANU philosophers—staff and graduate students—during my time as a VUW philosophy student and my many stints visiting the ANU. And cheers to all those who have commented on a draft paper of mine, asked me a question during a seminar or workshop talk, or just chatted to me about my research: your suggestions and criticisms over the years have been much appreciated. 


\section{List of contents}

Abstract 3

Keywords 3

Acknowledgments $\quad 5$

$\begin{array}{ll}\text { List of contents } & 7\end{array}$

$\begin{array}{ll}\text { List of figures and tables } & 10\end{array}$

Chapter 1: Music and philosophical naturalism—an introduction 11

1. Introducing music 11

2. Introducing naturalism 14

3. Introducing the thesis 18

4. Outline of the thesis 19

$\begin{array}{ll}\text { Chapter 2: Sound } & 27\end{array}$

1. Analyses of sound 28

2. Polysemy 32

3. The polysemy of "sound" 37

4. Audition and sound 42

5. Upshots and concerns 43

5.1 Upshots 44

5.2 Concerns 49

5.3 Conclusion $\quad 53$

Chapter 3: Conceptual pluralism about music 55

1. Overture 55

2. Concept pluralism: a sketch 61

3. Conceptual analyses of music 63

3.1 Reductive analyses of music 64

3.2 Multidimensional conceptualisations of music $\quad 71$

4. A plurality of music concepts 75

4.1 Two guises of music pluralism $\quad 75$

4.2 But we know it when we hear it! 80

4.3 Towards a philosophy of musics $\quad 82$

4.4 Conclusion 86 
1. A sketch of the science of music 87

2. Operational definitions of music 95

3. Pluralism in music-scientific debate 99

4. Coda: conceptual pluralism in aesthetics? 109

Chapter 5: Musical works 111

1. Musical works and ontological theorising 111

2. The musical work concept 116

3. The Canberra Plan 119

4. Ontological accounts of musical works 122

4.1 Nominalist/reductionist accounts of musical works $\quad 122$

$\begin{array}{ll}\text { 4.2 Abstract object accounts of musical works } & 125\end{array}$

4.3 Novel accounts of musical works $\quad 128$

4.4 Conclusion 131

5. Fictionalism about musical works $\quad 132$

5.1 Interlude: intentional inexistents 133

5.2 Revolutionary fictionalism about musical works $\quad 136$

5.2.1 Fictional statements internal to the musical works fiction

5.2.2 Fictional statements external to the musical works fiction

$\begin{array}{ll}\text { 5.2.3 The incredulous stare! } & 143 \\ & 147\end{array}$

6. Conclusion 148

Chapter 6: Evolutionary status of music $\quad 151$

1. Adaptation hypotheses 152

2. Non-adaptive by-product, exaptation, and cultural technology hypotheses

160

3. Lawson's "reconciliatory" evolutionary model 164

4. Co-evolution and niche construction 170

5. Towards a co-evolutionary, socio-cognitive niche model of music 176 
1. Hominin socio-cognitive evolution 177

2. Hominin evolution and the foundations of musicality 183

3. Stage One: the trackways-reading and tool-making pre-Homo 189

4. Stage Two: the Oldowan 198

4.1 Oldowan cognition, lithic technology and hunting 199

4.2 Oldowan musicality 201

5. Stage Three: the Acheulean 206

6. Summary 218

Chapter 8: Evolution of music $\quad 221$

1. Stage Four: the Late Acheulean 221

1.1 Late Acheulean technology 223

1.2 Late Acheulean fire culture 225

1.3 Late Acheulean proto-music 227

2. Stage Five: Mid/Upper Palaeolithic mythic/symbolic/oral culture 231

2.1 Music archaeology of the Upper Palaeolithic 236

2.2 Music in traditional/hunter-gatherer peoples 238

3. The final episode: the Holocene (Neolithic, Bronze Age, Iron Age, classical antiquity) 250

4. Concluding remarks 263

$\begin{array}{lr}\text { Conclusion } & 267\end{array}$

$\begin{array}{ll}\text { References } & 269\end{array}$ 


\section{List of figures and tables}

Figure 2.1: Enabling constituents of veridically perceived sounds (Vsounds) 43

$\begin{array}{ll}\text { Figure 3.1: Hamilton's music concept-space } & 72\end{array}$

$\begin{array}{ll}\text { Figure 3.2: Nercessian's music concept-space } & 79\end{array}$

Figure 4.1: Research areas along dimensions of temporal scale and organisation/abstraction

$\begin{array}{ll}\text { Figure 6.1: Siamang gibbon (Symphalangus syndactylus) } & 154\end{array}$

$\begin{array}{ll}\text { Table 7.1: Six key bio-cultural stages in hominin evolution } & 180\end{array}$

Table 7.2: Brain size/group size model predicted by social brain hypothesis $\quad 187$

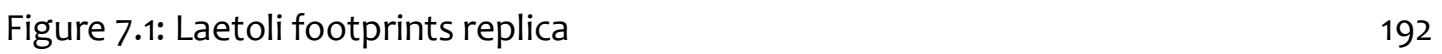

$\begin{array}{ll}\text { Figure 7.2: Acheulean handaxe } & 209\end{array}$

$\begin{array}{ll}\text { Figure 7.3: The evolution of human life-history stages } & 217\end{array}$

$\begin{array}{ll}\text { Table 8.1: Late Acheulean musicality } & 230\end{array}$

Figure 8.1: Cave hyena (Crocuta crocuta spelaea) painting found in the Chauvet cave 232

Figure 8.2: Replica of an Upper Palaeolithic flute from Geißenklösterle 233

Figure 8.3: Central African ivory horn $\quad 235$

Figure 8.4: African musical bow (Obubra, Nigeria), using the mouth as a resonator 237

Figure 8.5: A selection of Māori instruments $\quad 247$

Figure 8.6: Three musicians, from the Tomb of Nakht 255

Figure 8.7: Musicians and dancers, from the Tomb of Nebamun 256

Figure 8.8: Egyptian lute players, from the Tomb of Nebamun 256

Figure 8.9: Bronze chime-bells of King Yi's tomb 259

Figure 8.10: Music lesson painting on Ancient Greek vase, dated to around 510 BCE 260

Figure 8.11: Left hand panel (Ludovisi Throne): nude playing the aulos, dated to around

$460 \mathrm{BCE} \quad 261$

Figure 8.12: Hydraulis (water organ) and bukanē (curved trumpet) musicians 262 


\section{Chapter 1: Music and philosophical naturalism—an introduction}

\section{Introducing music}

Music is a ubiquitous and highly-valued feature of human social life. Musical traditions are deep-rooted features of every known society. Basic musical capacities are broadly distributed throughout the population; musical enculturation and awareness, broadly speaking, is a largely inevitable part of growing up in human society, building on phylogenetically deep capacities and, at least in early ontogeny, following a similarly structured developmental sequence across individuals. Yet across and within human societies, musical expression is incredibly diverse, as is musical style, music's roles in social contexts, and the ways in which people engage with music.

Music in the modern West alone testifies to this diversity. Some people are musical performance specialists. They train for hours every week, often from a young age, honing their skills over several years at a conservatorium before joining orchestras, chamber groups, jazz bands, or pursuing solo careers. Composers, conductors, even "mere" connoisseurs expend much effort on their craft or knowledge of the repertoire.

Other musicians form rock bands, singer-songwriter partnerships, electronic music groups, blues or folk bands. A few perform to tens of thousands of people weekly in stadiums, others to a hundred people in a bar on a Friday night, and others to whosoever walks past them on the street corner. Some musicians create jingles for adverts, others produce background music for shopping malls, sonic landscapes for art installations, or musical scores for films.

Most people are not music specialists yet are nonetheless musically engaged, part of their local music communities (of course, to varying extents). In the modern West, subcultural cliques are divided by, in part, their musical allegiances: consider hippies, goths, punks, ravers, metalheads, and hipsters. Many people put much thought into their chosen ring tones, even selecting specific tunes for particular friends. Congregations at churches, synagogues, temples and the like, come together to pray and praise through music. Sports fans cheer their team on through song and chant. Protest rallies engage onlookers through 
call-and-response. Military units entrain their drill marches to the beat of a drum. Christmas carols are near-unavoidable every December; no birthday celebration is complete without a rendition of "Happy Birthday". Parents and caregivers speak "musically" to babies and infants, with heightened melodic contour and emphasis on musical pitch. Lullaby, for example, is a common phenomenon both in the modern West and all around the world. And children learn through song. Music is an excellent mnemonic: think of the well-worn alphabet song. Teachers in New Zealand primary schools, for instance, obtain the attention of their pupils by clapping a conventional rhythm that is then clapped back in unison. Many children's games require music listening (e.g., musical chairs) or singing, often with movement ("Ring around the Rosie") or clap-chants ("Under the Bam Bushes"). And now, in the electronic media age, hand-held computer devices utilise educational applications and children's games with musical accompaniment.

Non-music-specialist adults express their musicality in myriad ways. Many listen to music whilst driving, some sing in the shower, others entrain to work-out music at the gym, others join in when musician friends are singing and strumming along around a campfire or lounge room, others hum along to the earworm currently circulating their mind's ear. Some hunters, trappers, birdwatchers, and the like whistle or vocally mimic calls in order to attract birds or animals they aim to spot, catch, or hunt. Though many adults consider themselves disastrously non-musical or even "tone deaf", only a very small percentage of people cannot entrain to a regular pulse, recognise a familiar tune, distinguish pitches (broadly speaking), or sing or hum along (even if quietly and unsurely) to "Happy Birthday" or a favourite song from their youth.

In short, the production and consumption of music, and expression of musicality, is not only the province of trained musicians and sound artists, it is very widely distributed throughout the whole human population. Indeed, as Gary Tomlinson puts it, 'Humans are musicking creatures' (Tomlinson 2015, p. 23).

Looking to the musics outside the modern West bolsters this picture of musical ubiquity and diversity. From gamelan ensembles to solo didjeridu or shakuhachi, to

\footnotetext{
'See Trehub and Trainor (1998) for an extensive discussion of childrens' play-songs, lullabies, and so on.
} 
music-induced vodou ritual trance, to the epic stories relayed generation after generation through song, to whole-group music-dances of contemporary and historic foragers, music clearly permeates human social life. There is a rich ethnomusicological literature, dedicated to documenting this pervasiveness, and the many roles that music plays in many different social contexts. Among the Igbo people of Afikpo, songs play many roles: from a form of derisive gossip-punishment for people's sexual transgressions (an outcome that lasts for years and would be dreaded by many individuals), to group celebration of a healthy birth (Ottenberg 1989). The stressfully-structured funerary practices of the Bara people of southern Madagascar, lasting several days, are offset by night-time singing and dancing to sexually-provocative, obscene songs (Metcalf and Huntington 1991). Among the Sherbro people of Sierra Leone, the beating of tree buttresses or up-turned canoes by members of the Thoma secret society signals the beginning of the ceremonial "rebirth" of society initiates — sounds that are then added to by drummers and female chanters (MacCormack 1980). And of course for many individuals the world over, music is a fun activity for simply passing the time and socialising. Anthropologist Frank Marlowe notes of the Hadza people, hunter-gatherer foragers living in northeastern Tanzania:

The Hadza sing often, and everyone can sing very well. When several Hadza get in my Land Rover to go somewhere, they almost invariably begin singing. They use a melody they all know but make up the lyrics on the spot. These lyrics may go something like "Here we go riding in Frankie's car, riding here and there in the car. When Frankie comes, we go riding in the car”. They take different parts in three-part harmony, never missing a beat, all seemingly receiving the improvised lyrics telepathically (Marlowe 2010, p. 67).

Music's cross-cultural spread and diversity, its multi-faceted nature, and social richness, mark it as an important and fascinating research target, ripe for serious investigation. And not only is music pervasive, it has a deep history, as I shall go on to explicate later in this thesis. Perhaps unsurprisingly, then, over the last twenty to thirty years in particular, music has become a subject for much specialised academic scrutiny 
(that is, outside that of typical Western music theory studies and musicology). A diverse research cluster has emerged, as I shall outline later in this thesis, in which researchers attempt to elucidate the nature and status of music, requiring contributions from philosophy, musicology, linguistics, psychology, neuroscience, evolutionary theory, anthropology, archaeology, and many other disciplines. This thesis is a contribution to that research cluster.

\section{Introducing naturalism}

Schematically, then, this thesis is a collection of arguments that together shed light on an interrelated cluster of concepts and issues, at the heart of understanding music, conceived as a natural explanandum. Indeed, this thesis could be thought of as a demonstration of a philosophical framework or agenda, as applied to the case of music. I use the moniker "philosophical naturalism" to describe this agenda, although I am mindful that the term has become rather en vogue and different senses of it crowd the literature. Nonetheless, my naturalism is central to this thesis, and I take it as a philosophical starting point.

As Papineau (2009) outlines, naturalism is typically understood as comprising an ontological component and a methodological component. As I see it, the ontological component is broadly physicalist/materialist. The naturalist eschews "spooky" entities, such as gods, immaterial minds/souls distinct from bodies, and non-causal abstract objects (for example, those supposedly populating the Platonic stratosphere of the Forms). Australian philosophers of the twentieth century have been particularly influential in this regard. Consider Jack Smart's appeal to identify the mind with the brain (Smart 1959), for instance, or David Armstrong's argument that mental states are physical states identified by causal roles (Armstrong 1968), or John Mackie's scepticism about objective values, in part given their "queerness" (Mackie 1977). The methodological component of naturalism seeks to tie philosophical and scientific methods. In short, philosophical naturalists of my ilk see philosophy as 'continuous with the natural sciences'; that is, we see naturalistic philosophical theories as 'conjectures whose fate is ultimately determined by empirical investigation' (Sterelny 1990, p. xi). 
This latter point is not to assert that there are no differences between philosophy and natural science, or how philosophers and scientists go about their research. To put it crudely, then, scientists typically study specifics-a particular virus, star, or chemical reaction-while philosophers typically ask questions of great generality, about causation, identity, knowledge, universals, and so on. Yet the philosophical naturalist insists that even such general theories are ultimately answerable to empirical data. Even rather specific topics in philosophy-Papineau (2009) names weakness of will, the importance of originality in art, the semantics of fiction—can be conceived by the naturalist thusly:

What seems to identify these as philosophical issues is that our thinking is in some kind of theoretical tangle, supporting different lines of thought that lead to conflicting conclusions. Progress requires an unravelling of premises, including perhaps an unearthing of implicit assumptions that we didn't realise we had, and a search for alternative positions that don't generate further contradictions... Still, methodological naturalists will urge, this doesn't mean that cogent empirical theories are not the aim of philosophy. An empirical theory unravelled from a tangle is still an empirical theory (Papineau 2009 \$2.1).

I am sympathetic. And I see this thesis, at least in part, as an opportunity to do some untangling.

Mark Twain once quipped that the trouble with the weather is that everybody talks about it, yet nobody does anything about it. Some philosophers, notably Elliot Sober, have voiced the same gripe about philosophical naturalism. Ontological naturalism has become a defining trend of much contemporary Anglophone analytic philosophy—many theorists across philosophical sub-fields are at pains to demonstrate that their theory or project is at least consistent with a minimally naturalistic worldview-yet much of this is mere lip service: few philosophers want to be called out as non-naturalists, or worse, supernaturalists. My thesis, however, begins with a call to philosophical naturalism. I examine issues concerning music from the perspective of a philosophical naturalist. This approach drives the thesis into some surprising areas of philosophy, as well as areas of research 
further afield. Understanding music is an interdisciplinary project, as shall be revealed throughout this thesis. My own attempt to shed light on music is, in part, a testament to this claim.

In my view, philosophical theorising can scaffold empirical investigation. Philosophy can contribute to genuine progress by synthesising research from the special sciences, uncovering connections, and revealing hidden prospects. Philosophical models can guide the search for new evidence, distinguish rival hypotheses, and weigh in on theoretical disputes. Moreover, philosophers are, in part, both conceptual engineers and dismantlers ("untanglers"). Philosophical work can clarify understanding of the natural world by unravelling complex theoretical frameworks or distinguishing conflated concepts. This task should be intertwined with empirically-oriented research-and so it is with my approach to music. For one, in my view, good definitions of phenomena under scrutiny can be research outputs as well as analytic or pre-theoretic assumptions or stipulations. (Wagner 2010, for instance, argues that the concept of adaptive fitness cannot be defined independently of empirical insight: good analyses of fitness are research outputs, not a priori stipulations.) For another, identifying merely verbal disputes and polysemous/pluralistic notions allows researchers to operationalise, and move relevant debates forwards. The contribution of philosophers to the levels of selection debate in evolutionary theory is one example of this: Sterelny and Griffiths (1999), for instance, distinguish two group selection concepts and show why the distinction matters to empirical debate. A second example is Mameli and Bateson's (2006) influential rounding-up of twenty-six definitions of "innate" and eight distinct innateness concepts put to use (and often conflated) by scientists. Diagnosing this situation is important. No researcher wants to waste time and effort in arguing past their rivals. And more crucially, once diagnosed, theorists can go on to identify the most critical issues at stake in order to see progress. An important part of Mameli and Bateson's ongoing innateness project sees the development of a research programme with the potential to vindicate the idea that the various definitions of "innate" are symptoms of an underlying (and curable) research clutter (see e.g., Mameli and Bateson 2011).

In my view, too much ink has been spilt in the service of essentialist-oriented philosophy. I am sceptical of the mainstream assumption in philosophy that, at least in many cases, properties of interest to us (such as, say, "catness" or "artness"—Papineau 
2009 discusses "ricketiness") are somehow "out there" in the world, waiting to be discovered by philosophers and straightforwardly formulated in essentialist terms: that is, captured by a concise set of individually necessary and jointly sufficient conditions that determine what it is for some $x$, real or imagined, to count as a cat, an instance of art, or a rickety automobile. ${ }^{2}$ In my view, we all (implicitly and explicitly) attempt to understand and categorise (or "carve up") the world—whatever it is that is "really" out there; whatever our best theories of physics/metaphysics might account for (objects, structures, processes, "real” patterns) —by way of our concepts and intuitions, procured experientially. This line of thinking has its roots in Hume's argument: that we project our concepts onto the world, imagining them to be part of reality's fabric (Hume 1748). Of course, some of these projections are more or less useful, and their utility is presumably a signal from the world. Yet all this often leads to conflations (polysemous terms and pluralistic concepts that require distinguishing and operationalising for use in pragmatic, practical, or scientific purposes) and to over-the-top abstractions (emergent concepts that are perhaps not plausibly realised by anything in the world, requiring an eliminativist or fictionalist rendering).

For now, this description of my philosophical attitude (naturalistic and broadly antiessentialist) may seem overly schematic. It will soon become clear how this informs my approach to philosophy of music. My intention here is to advertise my background assumptions/motivations. And rather than arguing for my agenda directly, I exemplify it in this thesis. Its validity and usefulness is best shown by its use. This schema has enabled me to address a broader range of topics than perhaps would have been possible to cover in a more conventionally-structured philosophy thesis, and this is a feature, not a bug, of my strategy: drawing connections between areas of research/theorising is necessary for progress, in my view.

\footnotetext{
${ }^{2}$ Consider, for example, arguments to the effect that there is a plurality of legitimate species concepts (see e.g., Ereshefsky 2010) that lead to multiple, non-equivalent ways to distinguish (say) cats from non-cats.
} 


\section{Introducing this thesis}

This thesis divides roughly into two overlapping sections; the division is partly methodological, but primarily due to content. On the one hand, the thesis develops a naturalistic philosophy of music framework, tackling semantic, conceptual, and ontological issues, concerning sound (chapter 2), music (chapters 3 and 4), and musical works (chapter 5). On the other hand, it offers a genealogy—a natural/social/cognitive narrative of music's deep evolutionary history (chapters 7 and 8) -its origins, expansion, and stabilisation. Thus this thesis is part philosophical analysis, in the traditional conception of that enterprise, and part evolutionary scenario building, with reflection on issues of evidence, theory, and methodology -an interdisciplinary project that spans philosophy and several sciences. Chapter 6, at the overlapping intersection of the two broad parts of the thesis, assesses hypotheses about the evolutionary status of music and the framework under which much of that discussion has taken place.

Understanding music's place in human nature (in the vernacular sense) cannot be achieved without a model of its (and our) evolutionary history: the ancestral stages giving rise to the present. Chapters 7 and 8 consist of an exposition, elaboration, and defence of a novel evolutionary model for the origins of music in the hominin lineage, synthesising research from a diverse cluster of disciplines and constructing a phylogenetically plausible (and empirically constrained) evolutionary scenario. As I explain below, the model departs from standard evolutionary theorising about music by rejecting the adaptation/by-product framework and focusing on bio-cultural co-evolution, dual inheritance, and niche construction.

A quick caveat before I outline the thesis in more detail. Evolution and philosophy have a long familial relationship, intertwined at least since Aristotle. Recently, however, some humanists have cautioned against the use of evolutionary theorising in human research. They allege various politically-unsavoury implications-unchecked scientism, crude genetic determinism, support for oppressive/racist "progressive" regimes, and a generalising "dehumanisation" of people and populations-although none of these are truly implied by any less-than-naïve contemporary evolutionary perspective as is well discussed by Colleran and Mace (2011). Post-modernist philosophers committed to the view 
that objectivity is impossible criticise evolutionary theory as yet another Western ideology driven by Western historical and socio-political factors, and is thus not epistemically distinctive or reliable. In this thesis I run with the assumption that these worries can be safely (and largely without controversy) put to the side. In my view, evolutionary theorising represents a rich enterprise for empirically-oriented philosophical research, as evidenced not least by the ever-growing intellectual output produced under the banner of philosophy of biology since the late 1970 .

\section{Outline of the thesis}

Here I summarise the aims of each chapter in a little more detail, and tie my research strategy explicitly to my philosophical naturalism.

Chapter 2 explores sound-intuitively the "mode" or "medium" of music. Various researchers have recently defended different, competing conceptual analyses of sound, and debate continues over the metaphysical nature of sound. The leading analyses either reduce sound to sensations or mental representations (proximal theories of the nature of sound), longitudinal compression waves (medial theories), or sounding objects or events (distal theories). Participants in this debate presuppose that because the features targeted by these reductive strategies are distinct (although related), at most one of the analyses is correct. In this chapter I argue that this presupposition is mistaken, endorsing a polysemous analysis of "sound". Thus the debate is somewhat misguided and largely merely verbal, or so I argue. All participants in the debate agree that there are the various physical reductions (waves, events, and so on), they simply differ on which of them "is sound". Yet there is no reason to think that, say, music psychologists studying sound sensations/representations, audio physicists studying sound waves, and anthropologists/ethnomusicologists studying sounding objects/events aren't just studying different reductions of "sound" despite the different explananda of their research. I do not think we need to choose between these explananda as sound. (By holding fixed the platitude that sounds are objects of audition, however, I am able to discount, for example, dispositional theories of sound.)

There is still substantial work to be done in the philosophy of sound. Theorists can still aim to explicate the best distal, medial, and proximal analyses: that is, debate over the metaphysical nature of sound events or objects, sound waves, and sound sensations or 
representations. Yet the various features of the world that are best played by the roles targeted by these theories can be utilised by researchers in different contexts, and in my view, philosophers need not choose between them for the "best" definition of sound simpliciter. So I conclude that the debate can be moved forward by disambiguating notions of "sound"-a broadly anti-essentialist conclusion-and I show how this strategy plays out by applying it to the debate on the ontological status of echoes.

In chapters 3 and $4 \mathrm{I}$ argue for conceptual pluralism about music. That is, while I argued in chapter 2 that the polysemous "sound" is reduced to terms for other conceptssound waves, events, and so on-with music I go further. Not only do I note the polysemy of "music", but the plurality of concepts of music. That is, not only is there no single, right answer to the question "what is music?" divorced from some angle or context, there are multiple, non-equivalent, legitimate music concepts. I argue that (1) unitary conceptual analyses of music are problematic, (2) there are good grounds for motivating conceptual pluralism about music, (3) taking pluralism seriously underwrites a fruitful research programme in philosophical aesthetics, (4) taking pluralism seriously has valuable upshots for the scientific study of music, providing a conceptual framework for integration and progress. (Although rewritten and extended by myself herein, these two chapters draw upon research undertaken in collaboration with Adrian Currie, of which I am the "corresponding author".)

Some curious, historical attempts to define (Western) music include sixteenthcentury theorist Nicolai Listenius's definition, 'Music is the science of producing melodious sounds correctly and well', and nineteenth-century modernist formalist Eduard Hanslick's definition, 'tonally moving forms' (see Goehr 2007, pp. 116, 164). Today, a commonly voiced definition, attributed to twentieth-century avant-gardist Edgard Varèse, is that music is organised sound (see Varèse 1966; Warner 2006). ${ }^{3}$ Slightly more promisingly, music psychologist Victoria Williamson tentatively defines music as 'a universal, human, dynamic, multi-purpose, sound signalling system' (Williamson 2014, p. 4). Nevertheless, taken as unitary definitions of music simpliciter (which, admittedly, they presumably were not

\footnotetext{
${ }^{3}$ Varèse was in fact distinguishing music from his own art: he was reconceptualising his own experimental musical output as "organised sound" rather than as music.
} 
intended to be) these definitions are all sorely simplistic. Williamson's definition picks out human speech and perhaps laughter (for example) just as much as it does music.

I consider three leading, sophisticated attempts to analyse, univocally, music - that of Irving Godt, Jerrold Levinson, and Andrew Kania-and I argue that they too fail as unitary, reductive analyses of music. To echo E. D. Hirsch Jr. on the definition of literature, my scepticism is aimed not at the existence of a music category", but rather 'at the attempt to falsely constrict its existence through definition' (Hirsch 1978, p. 26), at least, through essentialist definitions. I motivate conceptual pluralism about music by outlining pluralism's two guises-the plurality of music concepts utilised by researchers in legitimate research, and the plurality of ways that music is realised/instantiated, in practice, across the world's musical traditions. I focus on the former guise. (Music's cross-cultural variability is important but well established in the ethnomusicological literature, so less of a philosophically-interesting news item.) My discussions of a new research programme in aesthetics, and the upshots that pluralism has for the science of music, demonstrate the practical value of pluralism.

In chapter 5 I bring my pluralistic position about the music category to bear on the controversial topic of musical work ontology, perhaps the principal and most animated debate in contemporary analytic philosophy of music. I do this by honing in on one particular musical work concept-that of the classical Western art musical work; that is, the one that regulates classical art-musical practice (see Goehr 2007).

Thusly constrained, the "musical work" situation is revealed to be quite unlike that of "music" and "sound". I analyse the latter two as general concepts of categories, amenable to polysemy/pluralism. But in chapter $5 \mathrm{I}$ am honing in on one specific, univocal sense of "musical work"; that is, the one associated with the specific concept of the Western art musical work. Of course, on some occasions of use, the term "musical work" might be intended to pick out a performance or score copy—-these utterances are unproblematic insofar as the referent is a physical object or event. But when "musical work" is intended to refer above and beyond some physical counterpart, to some

\footnotetext{
${ }^{4}$ As discussed further in chapter 3 , categories are understood as an ontological rather than conceptual notion.
} 
purported entity in its own right—some specific object, some Western art musical workthat is where a new conceptual strategy is required for analysis.

In this regard I utilise a version of the naturalistically-respectable Canberra Plan methodology for approaching the ontology of (Western art) musical works. In short, my strategy outlines and combines the platitudes held by competent members of the relevant practice/discourse (the Western classical musical artworld) in order to explicate the relevant concept. Relying on such platitudes strikes me as particularly useful for determining the application conditions for a concept intertwined with a specific artful practice/discourse and history. This sets the stage for questioning whether the explicated concept is satisfied in the natural world, and what its ontological status might be. Philosophers have defended a bewildering array of ontological accounts of classical Western musical artworks. I provide a map of the intellectual terrain and argue that the accounts on offer either do not realise the role I have specified (that is, do not satisfy the application conditions) or posit metaphysically dubious entities (that is, suspicious to a naturalistic framework). In my view, it is not just that the jury is out. The development of a new theory is required. So I examine the prospects for a fictionalist view of Western musical artworks and I explicate fictionalist interpretations of the relevant musical work concept and discourse. Although much musical-work thought and talk can be plausibly reduced to thought and talk about concreta such as score copies, performances, ideas, and the like, I argue that talk of "the musical work such-and-such" should not be ontologically committed to some mind-independent entity. I argue that musical works are best conceived as intentional inexistents of a useful fiction. Somewhat metaphorically, they are "fictional characters", seemingly populating the Western art music tradition. In a manner of speaking, they can be objects of our thought and talk, and as such play a role in our artistic practice and musical discourse, but like unicorns, phlogiston, and Sherlock Holmes, I suggest, do not exist.

Given my music pluralism, though, I do not generalise from the case of classical Western musical artworks to other musical works. For instance, perhaps rock songs are best identified as "tracks" encoded on copies of recordings (that have a causal connection to the original master recording)—see Kania 2006; Gracyk 1996. In any case, I argue that 
the methodology I defend in the Western art music case will help philosophers, case by case, elucidate the ontological status of "works" of music across the board.

From here I leave chapter 5's specific focus on Western art music behind. I "zoom out" to consider the debate on music origins. Much of the philosophical and scientific debate surrounding the evolution of music concerns its status as an adaption, by-product, exaptation, or cultural technology. There is no shortage of theories in the literature. In chapter 6 I survey the key hypotheses with a critical eye. I ask: If the biological and cultural elements of music co-evolved, would these distinctions be undermined? Is there an alternative methodology for theorising about music's evolution? I argue the case for a dynamic gene-culture co-evolutionary, niche construction perspective. Moreover, I argue that taking this perspective seriously leads one to call into question the usefulness of the adaptation, by-product, exaptation, technology distinctions, central to the traditional (Santa Barbara-style) Evolutionary Psychologist's methodology, in evolutionary theorising about music.

The remainder of the thesis (chapters 7 and 8) develops a niche construction, gene-culture co-evolutionary/dual inheritance model of the evolution of music. My narrative is a bottom-up story of dynamic co-evolution and niche construction, explicating what we know about the evolutionary history of humans, and exploring behavioural tendencies through tracking between series of phenotypes in a phylogenetically plausible framework.

Indeed, despite the recent proliferation of evolutionary hypotheses about music in the scientific and philosophical literature, little effort has yet been spent on constructing evolutionary models that track between series of phenotypes-combining an evolutionary history of Homo and hominin cognition and sociality—for the purpose of constructing a phylogenetically plausible narrative that is compatible with contemporary interpretations of the evidence at hand; such a model of music would underwrite what philosophers of science call an inference to the best explanation. Kim Sterelny (e.g., 2012a, 2016a, 2017a), for one, attempts this kind of model in the context of human language evolution. Thisvis-à-vis music -is what I aim to do in chapters 7 and 8: I offer a narrative that emphasises the incremental evolution of music, vocal communication, gesture, sociality, and cognitive 
competences. Chapter 7 looks at the period in hominin evolution from roughly 4 Mya (million years ago) to 1 Mya, while chapter 8 focuses on the last million years or so.

Thus these final chapters present a positive picture of the evolution of music, integrating evidence from palaeoanthropology, archaeology, cognitive science, animal research, ethnomusicology, and evolutionary theory, among other fields. This picture is best conceived as work in the philosophy of nature. As Peter Godfrey-Smith (2014a) has characterised it, the philosophy of nature uses science as a resource for philosophising, rather than as a subject matter for philosophising about (as per standard philosophy of science). Projects in the philosophy of nature do not merely provide a philosophical report of the science; they interpret the raw science in order to motivate, put together, support and constrain an "armchair" empirical hypothesis: the kind of hypotheses Daniel Nolan classifies in the integration mode of the a posteriori armchair methodology (Nolan 2015). In this thesis, this includes reflecting on wider issues of theory and evidence, methodology, and application.

Moreover, these chapters present a niche construction perspective about hominin evolution. Hominins create and respond to environments made by earlier hominins; both individually and collectively, hominins have altered not only their physical and biological environments, for instance through the use of technology, but also their informational environment (see e.g., Sterelny 2014). Hominins alter not only their immediate environments, but that of future generations too. Adults teach the next generation skills, customs, norms; children develop and grow in an established informational world, scaffolded by social learning, which drives feedback loops and cultural innovation. So hominin evolution is, in my view, not best explained by standard "external" adaptationist models, but rather in reference to dynamic co-evolutionary forces.

Geoffrey Miller reflects that any theorist 'presenting a theory about human mental evolution is usually expected to present a speculative chronology of what evolved when, and to show how the current fossil and archaeological data support that chronology' (Miller 2001, p. 22). Yet some researchers have expressed disdain for the kind of evolutionary scenario model-building that I attempt in these chapters (e.g., Gould and Lewontin 1978). I think that their scorn is misplaced. As Sterelny (2012a) argues, suitably constructed, 
rigorous, evolutionarily plausible scenarios can identify possible pathways, 'showing that we do not need to posit exotic mechanisms or near-miraculous coincidences'; scenarios can 'narrow the search space... [identifying] broad areas of informational relevance, the materials from which tests can ultimately be built' (Sterelny 2012a, p. 2148). The construction and articulation of a plausible scenario is a difficult artform, made more challenging by the more empirical facts it is made to square with. Such narratives are far better conceptualised as rigorous and empirically constrained "how plausibly" explanations, not mere "just so" stories of wild imagination and free-flowing speculation.

Since this is a philosophy thesis there are a few methodological comments about my evolutionary model hinted at above that are worth drawing out explicitly. First, let me reveal two (obvious, perhaps) caveats: (i) my model is provisional. As is well rehearsed, 'the nature of the fossil record makes any interpretation preliminary' (Antón and Snodgrass 2012, p. 5485). New empirical findings can revolutionise theory-no one expected the discovery of the hobbits (Homo floresiensis), the Denisovens, or the genetic evidence of Sapiens/Neanderthal interbreeding. Moreover, admittedly, (ii) the model I espouse involves some degree of (constrained) speculation. Yet such models are required if we are to come to understand and explain the evolution of phenomena: we need worked-out theories that are clear and coherent, internally consistent, and compatible with the relevant evidence, which new evidence (when it comes to light) can either confirm or falsify. That is, evolutionary theorising of this sort proceeds through investigative scaffolding (Currie 2015) and Popper-esque hypothetico-deductive reasoning (Renfrew and Bahn 2012). Moreover, even if ultimately falsified by future empirical investigation, such narratives have practical utility. They have the potential to uncover connections that may not have been obvious and guide the search for new evidence and development of new research methods. And they remain valuable academic resources once recast as counterfactual models, allowing researchers to build improved and testable successors. ${ }^{5}$

So the evolutionary scenario developed in chapters 7 and 8 is, at least by necessity, research-in-progress. Nonetheless, I draw together findings and lines of evidence from multiple scientific fields and the resultant package is independently useful. As I argue in

\footnotetext{
${ }^{5}$ Daniel Nolan (2013), for example, provides eight distinct points of utility served by counterfactuals in the study of (pre-)history.
} 
relation to pluralism and musical science in chapter 4 , there is no one single discipline or field of musical science, rather a research cluster (Wylie 1999) of disparate agendas, methodologies, theories and evidence-generating techniques. Research clusters provide a messy, but promising route to understanding the phenomena under study-yet some synthesis and evolutionary model-building are required to scaffold progress, to get the elements in the cluster to interface, and to build explicit evidential connectionscontributions that philosophers are well-placed to make; that is, linking research and lines of evidence to theories and hypothetical/conjectural models from the various special sciences. I see the construction of narratives such as mine-and the philosophy of nature as I have outlined it above-as an indispensable endeavour for moving debate forward.

Adrian Currie thinks of this approach as methodological omnivory and it is not exclusive to the empirically-oriented philosopher: 'Rather than specialising in a certain kind of method, a certain array of tests, or a certain set of epistemic practices, historical scientists are opportunistic: drawing on whatever resources they can, at many levels of grain, to triangulate their way to plausibility' (Currie 2015, p. 188). Currie focuses on the evidence-generation of historical scientists. Though the philosophy of nature is, at least in part, in on the game of evidence-synthesis too.

So in my view, key roles that philosophers can play involve analysing and integrating work done in a truly interdisciplinary research cluster, and guiding the empirical search for evidence. Taking an evolutionary perspective on music can uncover connections that may have gone otherwise unnoticed as well as reveal gaps in both theory and methodology otherwise tricky to identify (as evolutionary theorising has done in research on, for example, menopause: see Shanley et al. 2007). My evolutionary model will demonstrate that some unobvious traces may be relevant to the debate, and reveal some connections that may not have been apparent from the viewpoint of any one discipline or theoretical perspective. Chapters 7 and 8, then, attempt to make the evolution of music debate more empirically constrained; more tractable; connecting it to a particular narrative of biological, cognitive and socio-cultural human evolution. 


\section{Chapter 2: Sound}

Music and sound are conceptually closely tied. As noted in chapter 1, a widespread commonplace characterisation of music is "organised sound". Conceptual analyses of music, which are explored in chapter 3, tend to couch music in terms of sound explicitly. Aesthetic evaluation and appreciation of a musical performance involves paying attention to sound: intuitively, sound is the mode in which music is manifest. And for some philosophers, the connection warrants even more force: 'music is the sound itself' (Hamilton 2009, p. 147). So before turning my attention to music in the next chapter, here the focus is on sound.

Until recently, philosophical research has focused on colour in debates about secondary qualities/sensible properties/the philosophy of perception. Growing unrest about the status of sound has recently generated a new literature (see e.g., Casati and Dokic 1994, 2012; Pasnau 1999; Nudds and O'Callaghan 2009; Bullot and Égré 2010a). One key question-what is sound? - dominates this literature and debate surrounding it is now lively. This debate is the concern of this chapter.

As I will outline shortly, the leading theorists in this debate identify sound with (1) sensations or mental representations, (2) longitudinal compression waves, or (3) sounding objects or events, presupposing that at most one of these three physical reductions is the correct analysis of sound. In my view, the presupposition that we must choose between these options is mistaken. I argue that each of these represents a bona fide reduction of "sound", which is best understood as a polysemous term. (A polysemous term is an ambiguous term with multiple interrelated senses-see e.g., Brusse 2016; Klein and Murphy 2001, 2002; Klepousniotou and Baum 2007; Sweetser 1986; Verspoor and Lowie 2003; Ravin and Leacock 2000.)

This chapter unfolds as follows. In §1 I take Robert Pasnau's influential article on sound as a starting point, and I consider the received view of sound that he identifies in that paper. That view has recently been the subject of criticism and has been labelled an incoherent view by some philosophers, including Pasnau. I go on to review the various 
theories held by contemporary commentators on the nature of sound, and I rehearse some objections to each as I journey through the theoretic landscape. These are useful to consider since they downplay the plausibility of a univocal analysis of sound, hinting that no unique reduction is plausible. $\S 2$ contains a schematic discussion of polysemy, and in $\S 3$ I motivate the view that "sound" is polysemous. In $\S 4$ I comment further on audition and veridical perception of sound. In $\S 5$ I consider several upshots of my view and I reply to some potential concerns. Of relevance to philosophers of music, my view implies that music-qua-sound is not uniquely identified with objects/events, waves, or sensations/mental representations-any of these reductions of "sound" can be the topic of thought or talk about music, depending on context, and we need not choose between them when discussing music in general.

\section{Analyses of sound}

Consider the following two theses:

The wave thesis: sounds are longitudinal compression waves; as such they are located in media through which they travel.

The audition thesis: sounds are objects of hearing.

According to the wave thesis, sounds require a medium through which to travel, such as air or water, and may be conceived as properties of that medium. And according to the audition thesis, sounds are things we hear. (Some philosophers take sounds to be the only things we hear; I intentionally leave this open in order to accommodate the possibility that we also hear silences-see Sorensen 2009.) According to Pasnau (1999), taken together the wave and audition theses constitute the received view of sound, which is in tension with a third thesis:

The reliability thesis: audition is generally veridical.

That is, we do not wish to ascribe massive, systematic perceptual error, all things being equal.

Together the wave, audition and reliability theses form an inconsistent triad. According to Pasnau, we usually perceive sounds as located at a place, somewhere in the 
distance, wherever the source of the sound is located. (For instance, according to Pasnau, we locate the sound of the birds chirping outside the window as being outside the window.) But if sounds are waves (and therefore in the air), then they are in fact all around us, yet all around us is not where we usually locate a sound. If sounds are waves, then, contrary to the reliability thesis, our perception of sound is incontrovertibly illusory. Accepting the wave and audition theses requires us to ascribe massive, systematic perceptual error.

So how should we proceed? Rejecting the audition thesis is problematic, given the revisionary implications of doing so. Audition tracks sounds. If one insists that it is not sounds but something else-call them "schmounds"- that are things we hear, then it is schmounds that we ought to discuss here. By adopting that strategy, then, we would be simply changing the terminology. The audition thesis identifies the target of inquiry. The possibility that we hear silences aside, as Warnock notes, 'we hear sounds... Perhaps we might say that the word 'sound' is the 'tautologous accusative' of the verb 'to hear" (Warnock 1982, p. 36).

Many commentators have recently rejected the wave thesis (e.g., Pasnau 1999; Casati and Dokic 1994, 2009; O'Callaghan 2009a, 2010; Matthen 2010) but not all (e.g., Sorensen 2009; O'Shaughnessy 2009). Four distinct sets of theories of sound have emerged—distal, medial, and proximate theories, as well as aspatial theories (for review, see Casati and Dokic 2012).

Distal theories of sound maintain that sounds are located at some place in the distance, from the perspective of the perceiver; that is, sounds are at or near the source of the sound. This view is the dominant view amongst contemporary philosophers writing on sound. Unsurprisingly, there are competing variants. First, that sounds are properties of vibrating objects or supervene on those vibrations (Pasnau 1999). Accordingly, sounding objects have sounds (rather than make them). Second, that sounds are events (individuals/particulars) that involve vibrations/collisions (Casati and Dokic 1994, 2009) or that, necessarily, disturb a medium, such as air, water or mercury (O'Callaghan 2009a). Third, sounds are dispositions of objects (Kulvicki 2008). Distal theorists can agree that 
sounds can generate sound waves in a medium —enabling generally veridical audition—but they maintain that sounds are not sound waves, rejecting the wave thesis.

I am not sympathetic to Kulvicki's dispositional account; it rejects the audition thesis as well as the wave thesis. Dispositions are not among the things that we hear. Even though the coffee cup in front of my monitor is presently stationary and seemingly silent, we may say that it has the disposition to vibrate in a certain way, to produce certain sound waves and consequently a certain sonic mental image in ordinary perceivers in normal circumstances if, say, I were to thwack it against my door. But I cannot hear this disposition, which on Kulvicki's view, is sound. The other distal theorists need not reject the audition thesis. ${ }^{6}$

Kulvicki's dispositional account raises a further issue worth making explicit about the audition thesis. Given that sounds are objects of hearing, they are occurent-not latent - things. In other words, sounds are of a so-called "temporary nature". They are fleeting. It might be thought that any plausible theory of sound needs to take this platitude seriously. So one potential objection available against property theorists like Pasnau (1999) is that, unlike the event theory, the property theory of sound does not adequately account for the intuitively temporal nature of sound (Casati and Dokic 2012). Events (distal), sound waves (medial), and sensations/representations (proximal), however, all fare well against this platitude.

Medial theorists advocate the wave thesis. Perkins argues that 'the sound we hear is identical with the train of airwaves that stretches from the distant sounding object to our ear' (Perkins 1983, p. 168). Similarly, Sorensen states 'I identify sound with acoustic waves' (Sorensen 2009, p. 140), and according to O'Shaughnessy, 'sound is identical with shock waves in a medium' (O’Shaughnessy 2009, p. 117). Mills defines sound as 'A physical concept concerning anything that can be heard resulting from vibrations in [media]' (Mills 2014, p. 243) cashed out in terms of frequency, wavelength and waveform. This view is dominant amongst theorists that privilege scientific descriptions of sound. It is assumed, for instance,

\footnotetext{
${ }^{6}$ One possible exception follows. The second arm of Pasnau's disjunct-that sounds supervene on vibrations-is apparently there to enable the possibility of dispositionalism (see Pasnau 1999, p. 316). If so, Pasnau allows the possibility of denying the audition thesis-a move that he is strongly opposed to in that paper.
} 
by the authors of Oxford's Grove Music Online entry on sound. ${ }^{7}$ Medial theorists do not deny the audition thesis—rather, they tend to attempt to mitigate the error ascription involved in rejecting the reliability thesis by calling into question just what it is for audition to be veridical, or by claiming that by perceiving sounds (that is, sound waves) one can locate the causes/sources in the distance, despite the sound being in the medium. As with the distal theories of sound, there are variants on medial theories, chiefly concerning whether sound waves are best conceived as processes or individuals. Most medial theorists conceive of sounds as individuals; the process option is less popular since it entails that sound waves do not move, but instead have different phases (temporal parts) at different places located in space, which is controversial (see e.g., Casati and Dokic 2012).

One objection that has been presented to medial theorists is this: sounds (when their sources are stationary) do not generally seem to be moving towards the agent perceiving the sound, even though the sound wave coming from (say) a loudspeaker to a perceiver is travelling through the air at roughly $340 \mathrm{~m} / \mathrm{s}$. The perceiver's aural experience would be very different if the sound instead passed through a multi-speaker setup, zooming from loudspeaker to loudspeaker towards the listener at $340 \mathrm{~m} / \mathrm{s}$ and seemingly entering her head 'like an auditory missile' (O'Callaghan 2010, p. 127). For an extensive overview of the merits of, and objections to, medial theories of sound, see Casati and Dokic (2012).

Proximal theories locate sound wherever the perceivers are. This view is dominant amongst psychologists, who view sounds 'primarily as constructions of the brain' (Bullot and Égré 2010b, p. 9) and as such, they are states or properties of perceivers. Proximal theorists disagree on the kinds of mental entities that sounds are said to be-auditory sensations, or sonic mental representations, or the like. Maclachlan, for instance, argues that sounds are sensations: 'sounds directly perceived are sensations of some sort produced in the observer when the sound waves strike the ear' (Maclachlan 1989, p. 26). According to Kandel, Schwartz and Jessell, 'We receive pressure waves but we hear words and music... [Sounds] are mental constructions created in the brain by sensory processing. They do not exist, as such, outside the brain' (Kandel et al. 1995, p. 370). And Levitin says that 'sound is a mental image created by the brain in response to vibrating molecules'

\footnotetext{
${ }^{7}$ See http://www.oxfordmusiconline.com/subscriber/article/grove/music/26289. Accessed 11/06/2014.
} 
(Levitin 2006, p. 24). Proximal theorists deny the wave thesis and the reliability thesis. If sounds are in our minds then they are not longitudinal compression waves and moreover, they are not where we usually perceive them to be; that is, somewhere outside our minds in the external environment.

Objections presented to proximal theorists appeal to purportedly counterintuitive implications of the theory, such as (i) if no perceivers are within auditory range of a collision then there is no sound-yet placing a perceiver nearby and replaying the collision produces a sound; or, expressed slightly differently, turn on a radio, leave the room, and the radio system ceases to produce sounds; (ii) in a seminar room in which perceivers hear only the speaker's voice, there are actually as many sounds in the room as there are perceivers; (iii) error ascription is misguided -after all, if I utter/da/ and you hear /ba/, you have correctly represented /ba/, which is the sonic image you heard. Another objection is that proximal theories do not account for our seemingly robust notion of distal volume. I can judge a screaming child to be very noisy despite being a fair way away from the child and consequently hearing it only faintly.

A fourth position is a kind of null hypothesis: sounds are aspatial and consequently, not anywhere (see e.g., Strawson 1959). This position is in tension with each of the wave, audition, and reliability theses and has been the topic of much critical assessment (see e.g., O'Callaghan 2010; Evans 1980; Casati and Dokic 2012). Moreover, aspatial theories are in tension with even a minimal philosophical naturalism if they "locate" sounds nonspatiotemporally. Given these tensions, it is a position of last resort and for my purposes will be discarded. Indeed, if my polysemy thesis can accommodate some of our intuitions or resolve some of the tension between the three theses (and I argue that it does), it is to be preferred over aspatial theories. (That is, I assent to the following theoretic-virtue thesis: if one theory of $x, T 1$, accommodates some of our intuitions about $x$ and another theory of $x, T_{2}$, accommodates none of them, then, all else being equal, $T_{1}$ is to be preferred over T2.)

\section{Polysemy}

So far, my discussion of analyses of sound has been brief and survey-like. The point has been to describe three plausible, broad accounts of sound—distal, medial, and proximal— 
and to note that debate continues regarding the metaphysics of the target of each account. I return in a moment to the topic of sound-I argue in what follows that "sound" is polysemous. However, first I must explicate some notions of lexical ambiguity and provide some examples of polysemy. I'll begin by contrasting homonymy with polysemy.

A homonym is a word type with multiple meanings. These meanings are distinct, unrelated and usually linked to separate etymologies. Which meaning is intended on a given occasion of use is usually clear from contextual cues. However, sometimes it is not. On some occasions of use, it might not be easy to tell which meaning of "bank", for instance, is intended—-that is, it could be tricky to disambiguate "bank", in some contexts.

The swimming champion noticed that the bank was crowded.

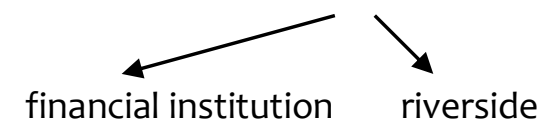

The same goes for some whole clauses that could be assigned multiple, distinct semantic structures not interrelated, like "He decided on the boat" and "They saw her duck and swallow" (see Zwicky and Sadock 1975). A speaker could obviously, on different occasions of use, intend to mean very different things with utterances of these sentences. The ambiguity is obvious to us when we read such sentences out of context.

Polysemy arises when a word's meaning has different linguistic senses; that is, the meaning of the word can be further disambiguated. ${ }^{8}$ Brusse offers the example "man": 'it can be variously used to refer to humankind as a whole, to male humans, or to adult male humans' (Brusse 2016, p. 94). For the following example, returning to "bank", assume that it is established that the speaker is intending to ask about financial banks, not riversides. However, to an interlocutor, it is unclear whether the speaker intends to ask after the

\footnotetext{
${ }^{8}$ I am following linguists' convention here: 'the different interpretations of a homonym are referred to as meanings, while those of a polysemous word are referred to as senses' (Klein and Murphy 2002, p. 548). This is a merely verbal distinction, wholly unconnected to the meaning/sense distinction made in the philosophy of language in reference to Fregean "senses" (and associated baggage).
} 
swimmer's preferred banking company, for example, or the swimmer's preferred building that houses a branch of such a business (see e.g., Klein and Murphy 2002). ${ }^{9}$

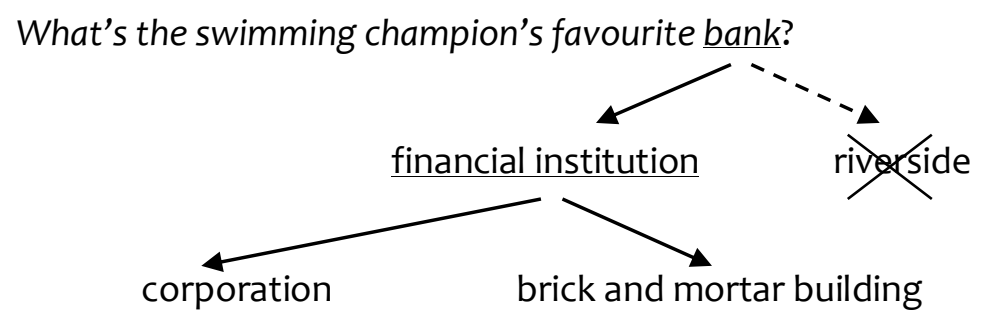

Consider another case of polysemy. William James, in his 1906-1907 lectures published as Pragmatism, discusses the example of a man and a squirrel both circling a tree trunk:

This human witness tries to get sight of the squirrel by moving rapidly round the tree, but no matter how fast he goes, the squirrel moves as fast in the opposite direction, and always keeps the tree between himself and the man, so that never a glimpse of him is caught. The resultant metaphysical problem now is this: DOES THE MAN GO ROUND THE SQUIRREL OR NOT? He goes round the tree, sure enough, and the squirrel is on the tree; but does he go round the squirrel? (James 2004, p. 21.)

Plausibly, the answer that one should give to James' question turns on what is intended by "go round"; that is, we must ask, which is the relevant sense of "go round" at stake? In my vocabulary, "go round" is polysemous; it has multiple senses that are closely related. If what is intended by "go round" is that the man passes from the north, to the east, to the south and to the west of the squirrel, then yes, the man goes round the

\footnotetext{
${ }^{9}$ To be sure, there is no appeal to the type/token distinction here: brick and mortar buildings that house a branch of a banking company are not being identified here as "tokens" of some "type", whether it be Bank, Financial Institution, or Banking Corporation. This could be done, I suppose, but it is not my point here.
} 
squirrel: the man occupies those successive positions as he circles the tree trunk to which the squirrel clings. But if what is intended by "go round" is being in front of the squirrel, then to the squirrel's right, then rear, then left, then in front again, then no: the man fails to go round the squirrel because his positions relational to the squirrel do not change.

There is no answer to the question of the man "going round" the squirrel simplicter, outside of some context-there is no "fact of the matter". But once the context is clarified (that is, the appropriate sense of "go round" disambiguated), it is straightforward to evaluate answers according to the relevant sense. 'Make the distinction, and there is no occasion for any farther dispute' (James 2004, p. 22).

Peter Godfrey-Smith (2014a) offers a similar analysis concerning talk of kinds. The idea is that "kinds" can be disambiguated into at least three related senses: sets, properties, and sums. Although the reality of each of these ontological categories has been called into question by philosophers, all that is needed for present purposes is to accept for now that theorising with these terms is 'acceptable in principle' (Godfrey-Smith 2014a, p. 109). Godfrey-Smith argues that biological species, which are traditionally conceived as kinds, can be conceived according to any of these senses, provided the notion is appropriately disambiguated in whichever context is at hand. The argument is made by analogy to several cases:

When I say "the Churchills" I mean the collection of people who made up Winston Churchill's immediate family... You can think of them as a set, with individual people as members. You can think of family as a sum, an object with parts. And you can think of the property of being a Churchill, which they all share. The three modes of grouping enable us to say different things. To say "The Churchill family left London" is to treat the family as a particular [sum], as something that can move through space. If you say "All the Churchills liked cigars" you are probably thinking of them as a set. But you can also say "It was being a Churchill that got him that job". Then you are using the language of properties. (Godfrey-Smith 2014a, pp. 109-110.) 
Another example is carbon dioxide,

You can say that dry ice is made of carbon dioxide (property); you can say that all carbon dioxide molecules contain 22 protons (set); and you can say that the $\mathrm{CO}_{2}$ in the earth's atmosphere has increased greatly during industrial times (sum). (Godfrey-Smith 2014a, p. 110.)

These senses of "kind" are interrelated because they are ways in which we have identified ("carved up") aspects of the structure of reality; they are "frameworks" for thinking about ontology, which can be further elucidated by appeal to other metaphysical distinctions (intrinsic versus extrinsic features, and so on).

One further example of polysemy: Brusse (2016) argues that "planet" is polysemous, and his argument shows that polysemous words need not be obviously so and that they can generate academic disputes similar to the debates concerning sound discussed in this chapter. Brusse's historical case study shows that the scientific term "planet" conceals multiple definitions with independent utility, due to the branching of scientific disciplines and subsequent independent evolution of the implicit definitions of "planet" within those disciplines.

I propose that "sound" is polysemous. ${ }^{10}$ Consider the following sentences. "The sound is located over there", "Sound travels at around $340 \mathrm{~m} / \mathrm{s}$ ", "I can still hear the sound of the crash in my mind's ear", "This sound is pitched higher than that one", "Veridical perception of sound in a vacuum is impossible", "Sounds have an intrinsically temporal nature", "Sounds are objects of audition". In these sentences, sometimes the most intuitive or charitable interpretation of "sound" is sonic mental images, sometimes sound waves, sometimes sounding objects/events. These are all different things—different physical reductions of sound-though they are closely related. Just as Godfrey-Smith demonstrates that talk of "kinds" can be talk of sets, properties and sums, depending on the context or what the speaker has in mind, it is my contention that talk of "sounds" can

${ }^{10}$ Obviously "sound" is also a homonym. As well as the relevant meaning of the noun "sound" to the present discussion (i.e., object of audition), "sound" can also mean fjord or sunken river valley, such as Milford Sound or the Marlborough Sounds, both in the South Island of New Zealand. 
be talk of mental images, sound waves, and objects/events. What's more, all parties to the dispute agree that there are sounding objects/events, longitudinal compression waves, and auditory sensations/sonic mental representations (despite dispute about variants within these reductions). This is more than what can be said for sets, properties and sums!

Yet theorists have not appreciated that talk of sound can be appropriately disambiguated - these reductions being the "cash value" of the term, to use William James' phrase. Consider the statement "sound travels at around $340 \mathrm{~m} / \mathrm{s}$ ". We intuitively understand this sentence, at first glance, to be about sound waves, and not about sounding objects/events or auditory sensations/mental representations, in much the same way that the statement "a man dies in a car crash every five minutes" is intuitively understood to be a claim about the frequency of male deaths caused by car crashes, and not about some unlucky punter who dies over and over and over again (Sennet 2011). The same can be said for the other statements concerning sound: a speaker's intended meaning-specifically, which reduction of sound is intended-is usually easily grasped by ordinary interlocutors.

\section{The polysemy of "sound"}

Consider the classic riddle: If a tree falls in an uninhabited forest, does it make a sound?

Like William James' squirrel case, this riddle is easily resolved by disambiguating "sound"; the answer turns on what is intended to be meant by "sound". If the relevant sense is sound waves, for example, then yes - the falling tree makes a sound since it generates a sound wave. If the relevant sense is auditory sensations, then no-after all, there are no perceivers in the vicinity. There is no answer to this riddle simplicter; no fact of the matter; yet, provide a context—make the necessary disambiguation—and giving an answer becomes easy.

When I look in the direction of a sounding object and say "the sound is located over there", I have a distal theory/sense of sound in mind. When I tell you about the vibrations of a guitar string I am talking about a sounding object or event. I am referring to a sound source. On the other hand, when I say "sound travels at around $340 \mathrm{~m} / \mathrm{s}$ ", I do so with a medial theory/sense of sound in mind. I speak of sound waves. And when I say "I can still hear the sound of the crash in my mind's ear", I appeal to a proximal theory/sense of sound; 
it is a sonic mental image or auditory sensation that I am referring to. When a friend complains about the ringing in her ears—something she apparently hears—she is telling you about a psychophysical sensation. (Perhaps it is the squeal associated with tinnitus.) Similarly, 'the voice we "hear" when we read fiction' (Deutsch 2000, p. 174) is some kind of sonic mental representation.

So we can speak of any of the things captured by the different accounts of sound using the language of "sound". Each sense of "sound" identifies a corresponding class of referents, and each can be thought of, in that sense, as sound. All interlocutors understand perfectly what is going on. Neither ordinary nor theoretic understanding of "sound" is challenged by the various uses in which speakers pick out referents that are ultimately captured by the different reductions of sound.

In sum, then, proximal theories of sound target sonic mental images or sensations, medial theories of sound target longitudinal compression waves and distal theories of sound target sound sources (sounding objects or events). There is no fact of the matter as to which one of these is sound, outside of some context. Now, this is not to say that previous commentators have been foolish to focus on one or another reduction of sound: debate can continue on the metaphysical nature of sound objects/events, sound waves, sound sensations/representations-for instance, on whether sound waves are best conceived as individuals or processes, and so on. What I deny is that one of these accounts-distal, medial, proximal-gives necessary conditions for something to be sound; I deny that one of these gives us the best account of sound simpliciter.

Moreover, it is worth noting the aims of the theorists who privilege one rather than another notion of "sound" in their research. Metaphysicians tend to favour analyses that reduce sound to objects/events, acousticians and other physics-oriented theorists tend to favour analyses that reduce sound to sound waves, and psychologists tend to favour analyses that reduce sound to sensations or mental representations. In my view, by analysing sound in the way that they do, these researchers could often be charitably interpreted as proffering an operational definition of the target of their inquiry—sound waves for audio engineers, auditory sensations for experimental psychologists, and so onstipulating which sense of "sound" they are using. This disciplinary divergence is similar to 
that of the case of "planet" (Brusse 2016). Jourdain pre-empts my point by noting that although physicists and psychologists both 'lay claim to the study of sound' (Jourdain 1997, p. 2) physicists study vibrations while psychologists study sensations; indeed, for Jourdain, whether sound exists 'independently of the ears that listen to it' (Jourdain 1997, p. 2) seems to depend on what is meant by "sound".

However, some theorists have tried to "lock in" the reference of "sound" by tying it to some essence-some essential conceptual feature; that is, 'an internal property that makes something what it is' (Godfrey-Smith 2014a, p. 112). Proximal theorists, for example, might say that phenomenal content/features are essential to sound, which are only located in the mind. What are we to make of the fact that philosophers on different sides proffer distinct essences? In my view, they are not providing an essential conceptual feature for sound simpliciter, but for the relevant sense at stake: in this case, that of auditory sensations/mental images. In other words, in stipulating that certain properties are essential to sound, philosophers are stipulating an analysis of sound for that theorist's purposes; it is to select and analyse one reduction. And that is fine, provided the sense of "sound" stipulated identifies a natural kind for the purpose of theoretical investigation worth doing, a criterion that is plausibly satisfied in all three of these general cases. Arguing over which of these is sound simpliciter looks to me like a classic case of a merely verbal dispute (see e.g., Chalmers 2011). Theorising about the various reductions-giving the best operational definitions of each, for instance-can and should progress, happily, side-byside. After all, it is the nature of the reductions that philosophers should care about, in my view, not which of these we best call "sound". And there is no need to privilege only one of these reductions, as sound, outside of any specific context. Indeed, the polysemy thesis dissolves the inconsistent triad. The wave and reliability theses turn out to be consistent so long as we appropriately disambiguate the term "sound" for the context (that is, sound wave and sound object/event, respectively). And I do not reject the audition thesis (the claim that sounds are objects of audition)—my polysemy thesis is that in different contexts, different things play the relevant role targeted by that concept (i.e., sound qua object of audition).

Proximal, medial, and distal theorists, at least in principle, can all agree with something like the following. Sounding objects such as vibrating strings (or events involving 
sounding objects), when in a medium, generate sound waves. Sound waves carry information of some description; they enable our generally veridical audition-they, in part, cause our auditory sensations/sonic mental representations (that is, when our internal perceptions are straightforwardly correlated to external counterparts: in some cases-e.g., the McGurk effect-our brain alters how we otherwise represent what is presented to us externally). Not all sonic mental representations are of something mind-independent. That is, some are not associated with a sound wave or a sounding object/event, but are purely mind-dependant (hallucinatory). But when our sonic mental representations are of something external, sound waves facilitate our generally veridical auditory perception, allowing us to track the sounding objects/events in the external environment responsible for producing the sound waves.

So theorists can all agree when we replace "sound" with the relevant disambiguation for the context at hand. Through audition we locate distal objects/events, via ascertaining from whereabouts a sound wave emanates through our brain's careful determination of differences in phase between our ears (see e.g., Nudds 2009). We aurally experience sounding objects/events as being located distally since our sonic mental representations have spatial content, although the sound waves that enable our veridical audition of sound objects/events are literally all around us, in the medium. The point is, as per James' squirrel case, make the relevant disambiguation of "sound" for the context at hand and all parties can agree (or not) on any residual theoretical or empirical issue at stake. (To illustrate, I discuss the example of echoes below in §5.1.)

So my ascription of polysemy should not seem uncharitable towards ordinary speakers (cf. Schiffer's criticism of DeRose's contextualist ambiguity thesis—an indexical view about "know" and its cognates-as hopelessly uncharitable towards ordinary speakers: see Schiffer 1996). Nor is it subject to Kripke's mocking ("lazy man's philosophy") of Donnellan's referential-attributive ambiguity thesis concerning Russellian definite descriptions, or of Hintikka's distinct senses of “know” (see Kripke 1977). The thesis that I am advocating is not linked to any philosophical term of art—knowledge, reference, existence, truth, consciousness, and so on-but rather to different ways that philosophers and ordinary folk alike can apply a common term; intuitively there is no reason to resist an 
ambiguity thesis in this case, despite Kripke's warnings. ${ }^{11}$ Indeed, as Hirsch notes, 'The only embarrassment to be suffered from the amorphousness of words is the attempt to pretend that they are not amporphous' (Hirsch 1978, p. 26). Indeed, the ubiquity of ordinary-term polysemy has, in recent times, become a hot topic in philosophical investigation. For example, Samuel Lebens (2015) in his article entitled “Would this paper exist if I hadn't written it?" argues that "this paper" is polysemous, and thus the answer to the question in the article's title turns on which sense is intended by "this paper"-the question can't be answered "yes" or "no" simpliciter.

Moreover, it is also worth noting that I need not conclude that we ought to change how we ordinarily speak. We would speak more clearly, and would avoid any potential "slippage of meaning", I concede, if we spoke of auditory sensations/sonic mental representations, sound waves, and sounding objects/events. That is, if we did not talk of sounds, but only of the reductions. (To be sure, we can imagine a hypothetical language that does not have a corresponding word for "sound" but rather only the reductions; the community speaking this language would be no worse off; they could still express everything that we can.) However, we are by no means speaking incorrectly by continuing to speak of sounds. And what's more, usually the context (and thus which interpretation of sound is intended) is easily discernible in ordinary discourse, so managing the polysemy in most cases is implicit and straightforward. Ordinary speakers know what they and their interlocutors intend to mean when they say, for example, that sound travels at $340 \mathrm{~m} / \mathrm{s}$,

\footnotetext{
${ }^{11}$ Consider Kripke's comments on Hintikka's purportedly distinct senses of propositional knowledge: ... what about the uses of "know" that characteristically are followed by that-clauses, knowing that $p$ ? Are these ambiguous? I would be very surprised to be told that the Eskimos have two separate words, one for (say) Hintikka's "strong" sense of "know," another for his "weak" sense. Perhaps this indicates that we think of knowledge as a unitary concept, unlikely to be "disambiguated" by two separate words in any language. (Kripke 1977, p. 268.)
}

Kripke takes his intuition to be evidential support for a single concept of propositional knowledge and not an ambiguity thesis. Would we be surprised to find a language that has separate terms for supposedly "weak" and "strong" propositional knowledge? Intuitively, probably yes. However, would we be surprised to find a language that has separate terms for the reductions of "sound"? Intuitively, no-not at all. The same is true of "bank" and its polysemous senses-a case in which ascribing ambiguity is uncontroversial. A further, methodological note concerning Kripke's criticism of ambiguity theses, is that I am not using ambiguity to "shield a theory from counterexample" in the way that Kripke charges Donnellan-rather I am rejecting the presupposition that only one account of sound can be right from the get-go. 
or that the sound of the birds is outside the window; we do not usually have to pause and check.

In short, in ordinary cases we can get along fine with talk of "sound". And in theoretic contexts it is easy enough to pause and make the disambiguation clear. This should help to avoid tangles, as philosophers continue to debate the metaphysics of sound events, sound waves, and sound sensations/representations.

In summary, then, the term "sound" and its cognates, I maintain, are polysemous. They can be used in different contexts to target different but related things on different occasions of use. The different disambiguated senses of sound are appropriate in different contexts. We do not need to choose between distal, medial, and proximal analyses of sound outside of some context.

\section{Audition and sound}

What do philosophers want a theory of sound for? Presumably it is to plug into theories of perception and to inform our metaphysics, generally speaking. This can still be done, of course, despite the polysemy of "sound". So I think it useful to consider a kind of empirically-oriented, practical approach, which will target veridical auditory perceptionthat is, veridically perceived sounds, or "Vsounds" for short-which in any event should be the test cases for any useful theory of sound. We want our theory of sound to capture the nature and causal relationships of the objects of our auditory perception. A standard case of veridical auditory perception necessitates that all manifestations of the senses of "sound" are present; that is, one's veridical perception of sound requires sound objects/events, sound waves and sound sensations/representations, and that there is no unnoticed or masked mis-match between any of these (resulting in auditory illusions or hallucinations). In other words, Vsounds are those in which an auditory perception is 'directly causally connected with an event consisting of the impingement of sound waves on the ear' (Rudner 1950, p. 384), regulated and interpreted in the right way by our (admittedly fallible) human cognitive/perceptual machinery. Puzzle cases for philosophical theories of perception, such as hallucinatory or dreamt sounds, and unheard sounds in uninhabited forests, are outliers and in any case, not Vsounds. 


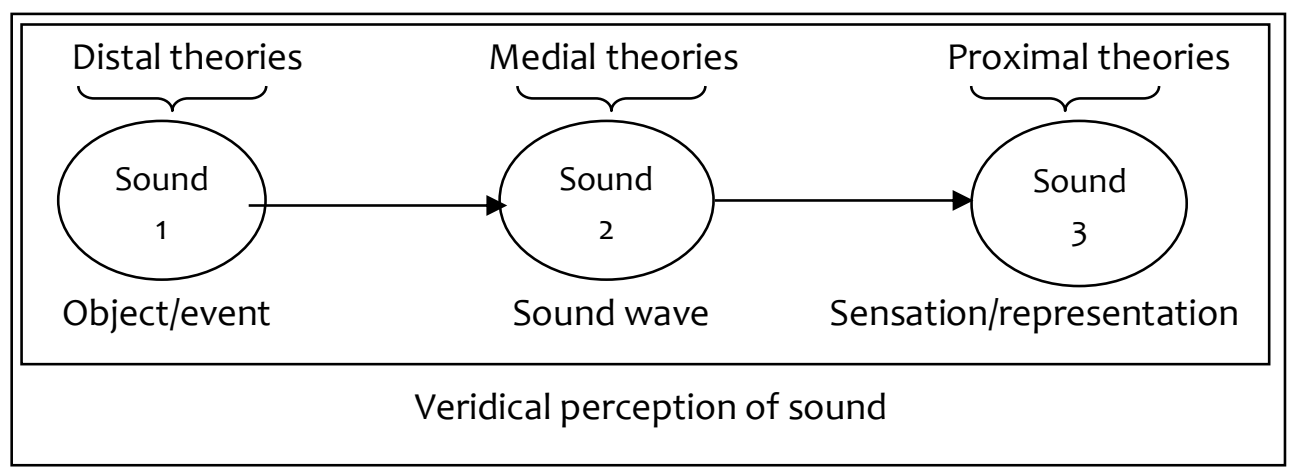

Figure 2.1: Enabling constituents of veridically perceived sounds (Vsounds)

So let's see how this straightforward, practical approach plays out by considering again that classic riddle, If a tree falls in an uninhabited forest, does it make a sound?

Disambiguate "sound" and (1) yes, there is a sounding object/event and (2) yes, there is a compression wave but (3) no, there is not subjective sensation or perception. Thus the answer to If a tree falls in an uninhabited forest, does it make a Vsound? is plainly "no". The physical counterparts of the distal, medial, and proximal reductions of soundhenceforth sound ${ }_{1}$, sound ${ }_{2}$ and sound ${ }_{3}$ respectively_-are required for Vsound.

Consider a couple of variants. By "veridically perceivable sound" I mean a potential Vsound: that is, sound ${ }_{1}$ and sound ${ }_{2}$ are present, such that if there were perceivers, they would (all things being equal) form sound 3 . The answer to If a tree falls in an uninhabited forest, does it make a veridically perceivable sound? is "yes"; there is sound ${ }_{1}$ and sound ${ }_{2}$, so if there were perceivers, there would be sound ${ }_{3}$ (but there aren't any, so there isn't). The answer to If a tree falls in a vacuum, does it make a veridically perceivable sound? is no; there is no medium for a sound wave to travel through (so no sound $\mathrm{l}_{2}$ let alone sound ${ }_{3}$ ). And so on.

\section{Upshots and concerns}

Since we need not choose between the distal, medial, and proximal reductions of sound as sound simpliciter, this leads to a Chalmers-style deflationism (see Chalmers 2011, p. 538), 
necessitating a shift of the locus of argumentation. That is, instead of asking "what is sound?", we look at the roles that we want sound ${ }_{1}$ sound $_{2}$ and sound 3 to play and figure out what plays those roles. Theorists can proceed by concentrating on the properties desired of the various notions of "sound" and looking to see what has those properties. ${ }^{12}$ Analyses of the reductions of sound should be shaped by the various roles targeted and best played by different properties. My polysemy thesis makes all of this overt. (And a theory of music can and should be relatively neutral about these specific theoretical issues about the metaphysical nature of the reductions of sound. ${ }^{13}$ )

For the remainder of this chapter, I discuss both upshots and concerns pertaining to my polysemy thesis of sound as outlined above. I then conclude, bringing the discussion back over to music.

\subsection{Upshots}

My polysemy thesis pushes the debate about sound away from some related issues regularly discussed in the philosophical literature about sound. For instance, theorists disagree on whether media are dispensable for sound or not. Clearly, those who accept medial theories will think that media are indispensible; sound waves do not exist in a vacuum. Distal theorists, for instance, are divided. Casati and Dokic $(1994,2009)$ and Pasnau (1999) think that media reveal sounds; that sounds exist in a vacuum but are not perceivable due to the absence of sound waves. O'Callaghan (2009a) disagrees; he argues that media are required for sound-he conceives of sound as an event that disturbs $a$ surrounding medium so his view is perhaps best characterised as a distal-medial hybrid, requiring sound ${ }_{1}$ and sound ${ }_{2}$ (i.e., similar to my notion of veridically perceivable sound, in $\S 4)$.

\footnotetext{
${ }^{12}$ For instance, sound's targets might include objects/events that involve vibrations, collisions, and the like. Sound,'s targets might involve that which has properties such as frequency, amplitude and harmonic-spectrum. Sound,'s properties might include pitch, loudness and timbre. Add a medium to sound ${ }_{1}$ occurring in a vacuum and you generate sound ${ }_{2}$, revealing frequency, amplitude and harmonic-spectrum; add a perceiver and you generate sound ${ }_{3}$, revealing pitches, loudness and timbres - and the full presence of a Vsound.

${ }^{13}$ Dyck (2016), to take one example, wishes to distinguish musical sounds from natural sounds via two conditions-yet he may sensibly remain neutral on sound (on conceptual analysis of sound, the nature of the reductions, and so on).
} 
Unsurprisingly, according to my polysemy thesis, whether sound exists in a vacuum depends on which disambiguated notion of "sound" one means. Of course, sounding objects/events can exist/occur in a vacuum—vibrating strings, bells being struck, and so forth. But no sound wave will be created. So there can be no auditory perception of a vibrating string or bell in a vacuum. Yet a perceiver, in a vacuum, could potentially have sonic mental representations, perhaps hallucinatory or electro-neural-induced ones (insert your own obligatory crazy-scientist thought experiment). ${ }^{14}$ Sound waves, obviously, require a medium. So if "sound" is polysemous, as I suggest it is, the debate over the dispensability of media for sound loses force. Ban "sound", and introduce "sound”,, "sound ${ }_{2}$ " and "sound 3 ", and then look to see where the disagreement is, if indeed any disagreement remains.

Similarly, theorists are also divided on what it would take to experience the same sound multiple times (see e.g., O'Callaghan 2009a, pp. 47-48). According to my polysemy thesis, again, it depends on which disambiguated notion of "sound" one means. One could hear the same sounding object (say, a guitar string) by hearing it plucked once and then hearing it plucked again. If the frequency of vibrations, force of the pluck, and all the other variables have not changed then the sensation/representation of the second pluck will, all things being equal, be another of the same type as the first pluck. Theoretically, one could hear the same sound wave by hearing it once, then racing ahead of it faster than the speed of sound waves, and hearing it a second time. In doing so, one will hear the same sounding object/event although the phenomenal quality might be different (it may seem quieter, for instance). One could re-hear an event by hearing the sound wave produced by that collision/disturbance echo off a reverberating surface, kindly '[saving] you the trouble of supersonic travel' (O'Callaghan 2009a, p. 48), yet costing you location perception displacement. Intuitively, one cannot re-hear a mental representation token, but at best form qualitatively identical iterations of a previous one (for example, replaying a memory of a sound event in one's mind's ear). Again, we can ban "sound", focus on the reductions,

\footnotetext{
${ }^{14}$ It is unclear whether electro-neural-induced representations that happened to map onto reality count as veridical representation by a non-standard route, or as hallucinations which happen to represent the world truly. I am inclined to suggest the latter which, I think, better preserves the notion of a Vsound, see $\$ 4$.
} 
and look to see where the disagreement is, if there is any. To explore this strategy further in finer detail, consider the debate over the ontological status of echoes:

Suppose you are at a fireworks display. You stand in an open field with a single brick building behind you. A colourful bomb's recognisable boom follows on the heels of its visual burst, but a moment later the boom's echo sounds at the brick wall behind the field. You have just heard a primary sound followed by its echo. (O' Callaghan 2007a, p. 403, emphasis in original.)

Casey O'Callaghan (e.g., 2007a, 2007b, 2009a, 2009b) advocates a view now dubbed the primary sound account of echoes (henceforth, PSAE). According to this theory, echoes are identical to their primary sound counterpart. Fowler (2013) formalises this theory as:

The Primary Sound Account of Echoes (PSAE): For every echo E, (i) there is a sound $S$ such that $E$ is an echo of $S$, and (ii) for every sound $S$ such that $E$ is an echo of S, E is identical to S. (Fowler 2013, p. 466.)

Recall that O'Callaghan is an event theorist of sound. His theories about "sound" are theories about events that cause a disturbance in a medium. So we should disambiguate "sound" as "disturbance event" when evaluating his views related to sound, such as his account of echoes. The PSAE should thus be read as the primary disturbance event account of echoes:

The Primary Disturbance Event Account of Echoes: For every echo E, (i) there is a disturbance event $D$ such that $E$ is an echo of $D$, and (ii) for every disturbance event $D$ such that $E$ is an echo of $D, E$ is identical to $D$.

As a listener perceives the same original disturbance event whether she perceives the primary wave engendered by the event or a subsequent wave reflected back off a surface, 
O'Callaghan's view about echoes plainly follows from his analysis of sound. Fowler (2013) attempts to reject PSAE, but he does not seem to take "sound" to mean "disturbance event", so his objections against O'Callaghan's theory miss their mark. Fowler provides hypothetical cases in order to attempt to call PSAE into question; here I will go through two cases and explain why they do not undermine O'Callaghan's PSAE as construed as a theory about disturbance events. The first case follows:

Chuck stands midway between a wall and a building scheduled for demolition, 100 feet from each, and Bryce stands 300 feet away from the other side of the building. Then the explosive charges fire and the building implodes, making the sound of a building imploding. A compression wave travels through the air to Chuck's ears and he hears the sound of the building imploding. The wave rebounds from the wall and soon reaches Chuck's ears again, causing him to hear an echo of the aforementioned sound. Simultaneously, the wave reaches Bryce's ears for the first time and he hears the sound of the building imploding, after which he is whisked away. (Fowler 2013, p. 467.)

Fowler's claim is that, given PSAE and the "temporal" nature of the property being an echo- that it is instantiated 'only after the associated compression wave has rebounded from a reflecting surface' (Fowler 2013, p. 467) —the sound that Bryce hears has the property of being an echo by the time he hears it; yet it is palpably absurd that Bryce hears an echo. This worry can be easily navigated. If Fowler is prepared to admit that being an echo is a temporal property, why not admit it as a locational property too, not yet instantiated at the place where Bryce is. The associated wave (or part thereof) where Bryce is propagates directly from the source/event-it has not reflected back off a reverberating surface. Moreover, re-reading Fowler's case study, disambiguating "sound" as "disturbance event", makes it plain that there is no cause for concern:

[...] A compression wave travels through the air to Chuck's ears and he hears the [disturbance event] of the building imploding. The wave rebounds from the wall and soon reaches Chuck's ears again, causing him to hear an 
echo of the aforementioned [disturbance event]. Simultaneously, the wave reaches Bryce's ears for the first time and he hears the [disturbance event] of the building imploding, after which he is whisked away. (Fowler 2013, p. 467.)

Chuck forms two numerically distinct mental images of the disturbance event. A compression wave, followed by its rebounding counterpart, relayed this aural information to Chuck, and were both originally generated by the same disturbance event, which O'Callaghan takes to be sound; this is what O'Callaghan means by the term "sound". In fact, make the distinction and it is now absurd for Fowler to deny O'Callaghan's PSAE. That is, by hearing an echo of, say, an implosion, one does not perceive a distinct implosion from the one that generated the primary sound wave-there's only the one implosion!

Fowler's second case:

On their upcoming trip to the Grand Canyon, Devon and Ellie plan to each shout 'Hello!' when they reach its edge. Before leaving, they make a bet: Each bets that they will hear more echoes of their own shout than of the other's shout. (Fowler 2013, p. 468.)

Fowler thinks that, given PSAE, neither Devon nor Ellie can win their bet if they both succeed in hearing at least one echo—since PSAE entails that all echoes are identical—and that that is absurd.

Clearly it is possible to hear more echoes of a sound if "sound" is cashed out in terms of sonic mental images or rebounding compression wave phases. This might account for Fowler's intuition about Devon and Ellie's bet. The bet is not best interpreted as about disturbance events per se, but about the behaviour of the compression wave created by that event, and how many times it ricochets off canyon surfaces resulting in perceptions or sensations in Devon and Ellie. Devon and Ellie each create only one relevant disturbance event here—Devon's shout and Ellie's shout—regardless of how many times compression 
waves bounce off surfaces and are subsequently perceived, so O'Callaghan's theory about disturbance events is not undermined by Fowler's intuition.

However, Fowler would be right to call into question a PSAE that disambiguated "sound" differently. PSAE is not a plausible account of, for example, sonic mental images. Most of the time, primary sounds and their echoes will have different phenomenal content, for example, locational properties (perhaps the first seems to come from in front of a perceiver, the echo from behind) or volume (perhaps the primary sound seems louder, the echo softer). Even when they might have the same phenomenal content, the mental images have distinct causal histories (one caused by a sound wave directly engendered by an explosion, the other caused by that sound wave ricocheting back off a surface). Since the two mental images have different properties, by Leibniz's Law they are distinct. So a primary "sonic image" account of echoes fails.

PSAE is also not a plausible account of sound waves. Fowler himself demonstrates this with an example concerning Chuck, who parachutes into a field just in time to come into contact with a compression wave that has rebounded back from a surface, but not in time to come into contact with the wave that propagated from the original implosion. Since the wave that Chuck perceives has reflected back off a surface, it has the property being an echo (qua wave). It is not identical to the wave Chuck missed, which lacks that property. By Leibniz's Law, they too are distinct.

In sum: all it takes to make sense of these puzzle cases is to disambiguate the notion of "sound" at play in the philosophical theory under dispute, in order to untangle and clear things up-in order for some progress to be made.

\subsection{Concerns}

One potential objection to my polysemy analysis is that problems seem to arise when terms for the various reductions of sound are substituted with "sound". Consider the following sentence:

(A) The sound wave produced by an ambulance approaches at around $340 \mathrm{~m} / \mathrm{s}$. 
Sentence A is true; it is an a posteriori discovery that (at least ordinarily) sound waves travel through the air at around 340m/s. However, if we substitute "sound wave" for "sound", we have a prima facie problem:

$\left(A^{*}\right)$ The sound produced by an ambulance approaches at around $340 \mathrm{~m} / \mathrm{s}$.

The objection here is that if "sound wave" is a term synonymous with a sense of "sound", substituting "sound" for "sound wave" should not affect the truth value of the sentence. Yet, the objection goes, sentence $A *$ is, according to our intuitive judgement, false: it is not descriptive of our ordinary experience of sound.

There are a number of things to say about this in response. The objection requires a reading of A* in such a way that reduces "sound" not to "sound wave", but to another reduction of "sound", perhaps "sounding object/event". We locate the sounding object/event at some place in the distance, and it would certainly not be approaching at $340 \mathrm{~m} / \mathrm{s}$, at least in ordinary circumstances. "Sound wave" and "sounding object/event" are synonyms for two different senses of "sound", but "sound wave" and "sounding object/event" are not themselves synonymous. So the objection presupposes a substitutivity principle that does not hold.

To show this, consider an uncontroversial example: "bank" in "What's the swimming champion's favourite bank?" Here, the word "bank" could be substituted for a synonym for the sense of "bank" in which the speaker intends to ask after buildings that house branches of a banking company, or banking companies themselves. However, no one thinks that buildings that house branches of banking companies are the same thing as banking businesses: the terms for the two different polysemous senses are not synonymous, so terms for these senses of "bank" cannot be substituted for each other with the expectation that the semantic structure of the sentence in question remains unaltered. "Financial institution" and "brick and mortar building" are of course obviously not synonyms. The same is true for the different senses of "sound". What's more, I have already conceded that we would speak more clearly if we spoke of sound waves, and so on, rather than of sounds. This is certainly true of sentences such as sentence $A *$. Yet this is no 
reason to resist my account. After all, it is an empirical discovery that sound waves travel at roughly $340 \mathrm{~m} / \mathrm{s}$-a surprising discovery, perhaps—so, little wonder then that, on the face of it, $A$ * seems intuitively false (and it is false, usually, of the other reductions of "sound"). So whether $A$ * is true or false turns on which sense of "sound" is intended on a given occasion of use, in my view. That is, we need only turn to the notion of speaker's reference. ${ }^{15}$ Substituting "sound wave" to "sound", after all, introduces an ambiguity. "Sound" is broader in scope than "sound wave".

On another note, one can ask: why is polysemy plausible in the case of sound in particular? More specifically, why do I evoke polysemy only in this debate as opposed to debates in various other philosophical domains? Wouldn't an appeal to polysemy settle the long-running dispute on colour, which in many ways is the older sibling of the dispute on sound? And why not moral theory—maybe "good", "right action", "ought" are polysemous?- -and so on. Well, maybe: but each case for polysemy must be judged on its own merits. It needs to be defended on a case by case basis. There are five things to note on this point.

First, I am interested in the debate on sound since I am interested in music. Exploring the prospects of polysemy analyses in philosophy generally is beyond the scope of this thesis.

Second, recall that all parties to the dispute agree on the reality of sounding objects/events, sound waves, and auditory sensations/representations. There is no such agreement in, say, moral theory: parties to the dispute typically do not agree, for example, that there are moral facts both of the Kantian sort and the consequentialist sort, which various purported senses of "good" could, once disambiguated, lock in on. (And it is not plausible that such purported senses are anchored in different research traditions and

\footnotetext{
${ }^{15}$ If the speaker intends to mean sound waves, then all things being equal, $A$ * is true, insofar as $A$ is true. And applying a principle of charity, we should suppose that the speaker does intend to mean exactly that in uttering $A^{*}$; that is, at least in the absence of any reason not to suppose that (since it would be incredibly unusual for a speaker to utter $A^{*}$ otherwise).
} 
questions, in the way different versions of "sound" are.) Evidently, without additional work, polysemy may not appear a good strategy for dealing with moral theory. ${ }^{16}$

Third, and as explained already, I acknowledge that there is genuine substantial disagreement within the philosophy of sound: theorists disagree on the nature of sound, sound $_{2}$, and sound ${ }_{3}$. Insofar as they do there is still progress to be made. And recognising the polysemy will help to progress and avoid conceptual tangles, as I have shown in the debate on echoes.

Fourth, even if my ascription of polysemy works for sound, it may or may not for, say, colour. That is yet to be argued. Historically it has been asserted that, just as sounds are proper objects of hearing, colour is the proper object of vision (e.g., George Berkeley, John Locke on secondary qualities), however I think this is misguided. What would Warnock's "tautologous accusative" of "to see" be? If sounds are proper objects of hearing (as per the audition thesis) then sights are proper objects of vision. That is, it is the look of thingsnot merely the colour of them-that is the parallel explanandum. And "sights" may well be a candidate for a more-or-less parallel polysemous treatment: visual sensations/mental representations, the electromagnetic energy events that generate light waves, lightreflecting properties of objects/events, and so on. However as far as I can tell, there is no philosophical debate about "sights". Perhaps participants in the debate about sound could learn from this.

That said, if the analogy from sound to colour is more straightforward than I am suggesting, since at least part of the debate on colour is similarly structured (with distal, medial, and proximal theories), I would indeed be sympathetic to a polysemous analysis of "colour". But the proof would be in the pudding.

Finally, "sound" does not seem to pick out anything in addition to the physical reductions. Contrast this with another case (which we will explore later, in chapter 5). Most parties to the debate over the ontology of musical works agree, for instance, that a Western art musical work is something else in addition to the various attempted physical

\footnotetext{
${ }^{16}$ Richard Joyce has pointed out to me that polysemy may be useful in metaethics. He suggests that perhaps "moral fact" has (at least) two senses, one requiring objective prescriptivity and the other not.
} 
reductions (scores, performances, recordings, playings, mental representations, and so on); indeed, a 'ghostly apparition' (Kivy 2002, p. 206). In a case like that, it is not clear that polysemy is at work, however the situation with "sound" is not like that.

In sum: the conditions are right for evoking polysemy in the debate on sound. It may be available in other domains too (James' "goes round" case; Godfrey-Smith's "kinds" case; Brusse's “planet” case; Chalmers' merely verbal disputes), but a full exploration of the prospects for polysemy is not my task. Suffice it to say that polysemy is, at the very least, plausible concerning the present discourse (and in my view, extremely intuitive). Indeed I take my argument throughout this chapter to at least give good reason to find rejecting the presupposition that only one of these reductions are objects of our audition an attractive move. The various senses of "sound", each in some respect, capture what we hear-each corresponds to a real-world thing necessary for ordinary veridical aural perception. Picking any one of these as sound (simpliciter), faces a charge of ad hocery, it seems to me. So the ball is in the court of the essentialist theorist-whether proximal, medial, or distal-to explain why only one reduction of sound can be an object of audition, when all are required in veridical auditory perception and when all theorists agree on the existence, importance, and general relationship of the three candidate reductions: as I suggest in $\S 4$, if anything it is Vsounds that we should privilege as the objects of our (veridical) audition in our theories.

\subsection{Conclusion}

The different sets of theories described in §2 each target different reductions of sound. There is no overriding fact of the matter, outside of context, that settles which uniquely is sound; no reason to privilege any one of them as sound simpliciter. Theorists advocating a univocal distal, medial, or proximal analysis presuppose that because the properties targeted in the analysanda of the various analyses are distinct, at most one is correct. I maintain that this presupposition is mistaken. I have shown how this can shed light on related controversies, such as the debate about the ontological status of echoes.

And this has implications for how we conceive of music (qua sound). Thought or talk about musical sound can be about the relevant musical event(s) or object(s), the sound wave(s), the mental image(s) or auditory sensation(s). There is no need to "locate" music 
uniquely in any of these domains-in the medium around us, or in our heads, for example. On this, Cross notes:

Music, as sound [waves], appears susceptible to explanation in terms of physics...; as perceived sound, it should be capable of being elucidated by means of psychoacoustics...; and as [patterns of action] that cannot be derived directly from the physical signal and that yield pleasure, at least some aspects of it should be understandable from the perspectives of the cognitive sciences and neuroscience. (Cross 2012a, p. 315.)

Music is often described in terms of pitch, loudness, and timbre. When this is the case, we are speaking in the language of sound 3 ; we are speaking about musical experience (perceptions, sensations). This is a rather common usage of the language of music (qua sound). However, music could also be described in terms of frequency, amplitude, and harmonic-spectrum, for instance by audio engineers and acousticians, in the language of sound $_{2}$. Or indeed in terms of vibrations or collisions, in the language of sound ${ }_{1}$, although this may be less common in ordinary parlance. However, for instance, the imperative statement "Turn that music down!" might be best interpreted as an instruction to influence the sound event by changing the settings on the stereo loudspeakers-the source of the sound. The point is, upon reflection, it is easy enough to make the necessary disambiguation.

In the next chapter I turn directly to conceptual analyses of music. There I advocate a form of conceptual pluralism about music. That is, while the polysemous term "sound" is easily reduced to terms for other concepts—events, waves, and so on-not only is "music" polysemous, it involves the engineering and identification of multiple, non-equivalent concepts of music (i.e., conceptual pluralism). ${ }^{17}$ In a slogan: "musics, not music!"

\footnotetext{
${ }^{17}$ Thus as I have described the case of sound, it fits the semantic characterisation of "fragmentation" rather than "pluralism", at least as I see it (Brusse 2016).
} 


\section{Chapter 3: Conceptual pluralism about music}

Some cultures make music by banging on drums, blocks, pieces of metal: they value rhythm (and perhaps timbre) above melody. In others, the main instrument is the human voice; in others, music is inseparable from dance. In some cultures, music is reserved for special occasions; in others, people create a more or less continual musical soundtrack to their lives. Some reserve a term like 'music' only for a subset of the apparently musical things they do... Perhaps most significantly, there is no reason to suppose that all musics should share any particular trait in common-that music has universal features. As semiologist Jean Molino has put it, 'Nothing guarantees that all forms of human music contain a nucleus of common properties that would be invariant since the origination of music'. (Ball 2010, p.10).

\section{Overture}

Music is multi-faceted, and it manifests in extraordinary heterogeneity. A musical performance's pitch structures, for instance, varies from the standard (Western) modes, to enigmatic whole-tones scales and edgy octatonics, to distinct Javanese pathet and Indian räga-all of which diverge both in formal structure and expressive range. Likewise for rhythmic schemes, dynamic ranges, and timbral families. Moreover, music plays many roles. Music delivers emotional catharsis, provides ceremonial backdrops, pedagogical and ritual props, social glue, individual inspiration, and entertainment.

As scientific and humanistic investigation proceeds, researchers develop new and empirically testable theories of the mind, models of evolution and development, hypotheses about the arts and culture, and interdisciplinary connections (e.g., neuroaesthetics). Given music's cross-cultural ubiquity and deep historical entrenchment, the many roles it plays in myriad contexts, its internal complexity and extraordinary 
diversity in its manifestation, it is unquestionably an aspect of human life requiring explication and understanding. This much is uncontroversial. And in order to research music it would be a great help to have a very clear idea of what it is researchers are investigating. So how should researchers conceive of music? How should they delimit their object of study? The territory here is genuinely contested: should researchers include, as music, unintentional performances in certain contexts? Should they include ritualistic chants or housie calls? Should they include performances of John Cage's 4'33"? And if researchers' aims are not to cover all phenomena ordinarily conceived as "music", then which should they cover? And what legitimises their music/non-music distinctions? These questions are easier asked than answered. Here I aim to show how answering them, in a meaningful and defensible way, might proceed.

To be sure, music is elusive-difficult to pin-down and define (Davies 2012a). It is my contention that music's elusiveness and multi-faceted nature runs deep. That is, in my view, there is no fact of the matter about what music "is", tout court, divorced from some angle, cultural tradition or research interest. In this chapter, I take up Jean Molino's nonessentialist sentiment (nicely captured in this chapter's epigraph, from Philip Ball). I argue for conceptual pluralism about music.

According to pluralism, at least two non-equivalent (that is, not extensionally equivalent), equally legitimate concepts apply to some category. I argue that this is the case for music. Here, "category" pertains to ontology: a way that the world is carved up. A concept is a function from states of affairs to a category. A concept determines category membership - a music concept, then, decides which candidates count as music and which do not. A music monist is someone who thinks there is a unitary music concept-the concept of music —and thus a tout court correct answer to the question "what is music?", which is of course denied by the music pluralist. The pluralist argues that music (qua category) can, under different circumstances, be carved up in non-equivalent, though meaningful and legitimate ways, by a plurality of music concepts. Indeed, the relationship may often be one-to-many: a single instance of music might instantiate several distinct music concepts concurrently. Others might instantiate fewer. 
My argument for pluralism about music could be taken as a case study for aesthetic concepts generally; I suggest that pluralistic concepts have the potential to underwrite fruitful research directions in philosophical aesthetics. My approach differs from typical philosophical approaches to music concepts. Philosophical analyses of music typically take one of two forms: reductive analyses encompassing individually necessary and jointly sufficient identity conditions, or multidimensional, though unitary, conceptualisations comprising "salient features" (i.e., a unified "family resemblance" approach). I argue that these unitary approaches have failed, and this failure, in part, motivates my pluralism. Moreover, pluralism about music, as I discuss it, underwrites a potential research programme for aesthetics. Participants in this programme will distinguish research interests as well as characterise-perhaps design_-and critique concepts of music suitable for those interests.

Jonathan McKeown-Green (2014) recently argued that the intuition-driven methodology prevalent in the philosophy of music (and aesthetics more generally) is problematic on the basis that what counts as music simpliciter is not narrowly determined by how any one person (or group of people) conceives of it. According to McKeown-Green, then, music cannot be captured by our current conception because conceptions change through time. ${ }^{18}$ This implies that what counts as music at least intuitively-and thus the intuitive/folk music concept that underwrites that delineation-is dynamic. What we in the Western world intuitively count as music today has diverged from what our great-greatgrandparents counted as music 200 years ago. What will count as music 200 years in the future is anyone's guess. This suggests that one salient conception of music picks out a connected lineage of practices.

Music concepts can also be indexed to particular cultures or folk settings. This point is well accepted in the ethnomusicological literature (see Nettl 2005). As Curt Sachs writes, 'our generic term music is misleading. For it implies an all-embracing concept of the various elements that reach our ears and therewith a common rule for each of them' (Sachs 1962, p. 193). To this end, much ethnomusicological research targets the cross-cultural diversity of music (not to mention the variability within particular cultures-see e.g., Rzeszutek et al. 2012). While Western music scholars might be tempted to analyse music wholly in terms of

\footnotetext{
${ }^{18}$ Lennox (2001) argues for a similar position on observation in the history of science.
} 
melody, harmony, meter, and the like, 'there are societies and musics where these criteria make no sense at all' (Nettl 2005, p. 18), let alone an abundance of Western examples that lack at least one of these criteria. Yet for some investigations, restricting discussion to instances of music with particular intrinsic features may be warranted, without concern as to whether they belong to a suitably "musical" lineage of practices. ${ }^{19}$

Following the empiricist leaning of Jesse Prinz among others, as functions from states of affairs to categories, 'concepts are copies or combinations of copies of perceptual representations' (Prinz 2002, p. 108). It's no surprise, then, that concepts of music will be different for people from different musical traditions, societies, or historic eras - their perceptual experiences will reflect the musical system (or systems) as well as the informational processes that scaffold their experience. The idea is that thinking about some music concept necessitates activation of representation in aural circuits, visual circuits, tactile circuits, motor action circuits, and so on-this requires experience of some kind. In a way, the different musical traditions of the world provide distinct inputs for such a process, resulting in different outputs, and differences in cultural significance of music (such as ties to ritual and/or religion) will involve differences in internal functional organisation.

My pluralism claims that not only is "music" polysemous in nature, but that there are a plurality of music concepts, and what counts as a legitimate music concept depends upon context (and not merely conceptual context). On my view then, it is disunified diachronically, as McKeown-Green indicates, but also synchronically, as ethnomusicologists such as Sachs point out. My view gives rise to a methodology which utilises traditional philosophical tools (conceptual analysis, for example), but is not vulnerable to McKeownGreen's apprehensions.

The discussion so far has perhaps gestured towards at least two conceptions of music. I shall dub these the intrinsic and relational conceptions. (This is a loose usage of those terms not meant to imply that intrinsic conceptions do not have relational aspects and vice versa.) The intrinsic conception of music picks out music by (perceptible) resemblance, perhaps with respect to standard features like rhythm, pitch, harmony, melody, and perhaps other constitutive performance features such as intention. The

\footnotetext{
${ }^{19}$ See Beban (2001) for an ethnomusicological investigation of auction and betting chants.
} 
relational conception of music picks out music as a part of an institution, tradition or lineage of practices: it picks out causal connection rather than similarity of elements. There is much overlap between these two conceptions, of course. However, philosophers analysing music have focused on the intrinsic conception (as we will see throughout §3). My discussion here will largely reflect that bias: note that the purpose of this chapter is to motivate and defend music pluralism generally, not to vindicate (or debunk) specific music concepts (though I discuss how a philosophical research programme could proceed in that respect in $\S 4.3$ ). In my discussion of musical works in chapter 5 , and in my evolutionary analysis of music, in chapters 6 through 8 , the bias obviously shifts to relational conceptions of music. As will become clear, what vindicates a pluralistic adoption of music concepts is the legitimacy of the research agendas underwriting those concepts and the extensional non-equivalence of those concepts.

Thus I go beyond merely attributing polysemy to the word "music". In the previous chapter, I argued that "sound" is polysemous: that sound can be reduced to various physical counterparts depending on context—sound event, sound wave, sound mental image. Participants in that debate tend to assume that only one of those reductions is sound, but all agree that all the reductions exist and that there is no additional thing-so no new sound concepts needed to be engineered. The relevant concept targets objects of audition, this is reasonably uncontroversial; my polysemy thesis is that in different contexts, different things play the relevant role targeted by that concept. I diagnosed that polysemous situation as one of fragmentation, in Brusse's (2016) sense. That situation, however, is not the case with the polysemy of "music", at least, not the full extent of it. Here things are not so straightforward-I argue that there are multiple music concepts at stake. There are musics, not music.

I begin, in §2, with a general and schematic discussion of conceptual pluralism: what does a pluralist claim, and how might she justify herself? To illustrate, I provide a short example of conceptual pluralism from the philosophy of biology literature: species pluralism. 
I then move on to my argument. In $\S 3$, I discuss the failures of the traditional approach to music concept analyses. I begin by critiquing three leading unitary analyses of music - that of Irving Godt, Jerrold Levinson, and Andrew Kania. I then argue that any reductive unitary concept of music will either admit dubious cases, be hopelessly ad hoc, or completely unwieldy. Some philosophers have attempted an alternative approach, aiming to capture music across a set of dimensions that attempt to identify music's salient features. This can be conceived as a kind of "family resemblance" approach. I argue that although this approach has its attractions, I question its usefulness as a unitary approach. Which dimensions are admissible seems to depend on the investigative programme or explanatory interests of the theorist, and the salience of the dimensions also seems to vary from case to case (as plausibly would be the case were we analysing the paradigm example of "game").

This leads me to pluralism in $\S 4$. Therein I elaborate my position, distinguish between some possible legitimate, non-equivalent music concepts, and respond to the music monist's objection. I argue that pluralism provides a fruitful and useful avenue of philosophical research. Pluralism, then, is underwritten by the failure of unitary approaches, the plausibility of pluralism, and pluralism's fruitfulness.

Yet the buck does not stop there. In chapter 4 I explore the upshots of pluralism about music for contemporary scientific and theoretic debate. The world's musics are natural phenomena that can be studied empirically, as can the capacities that enable music's production and appreciation. I argue, based on an examination of musical science itself, that music pluralism is plausible - that given the range of questions and evidence which scientific investigations of music requires, there is no unitary definition of music. I also argue that musical pluralism can lead and drive integration in the scientific study of music. I assess several operational definitions proffered by researchers, and distinguish complementary relationships between definitions from antagonistic ones (Currie and Killin 2016). Complementary definitions target different aspects of musics or music in different contexts; antagonistic definitions give rise to empirical disagreements and should be resolved. I finish by tentatively considering pluralism's prospects in aesthetics more widely. 


\section{Concept pluralism: a sketch}

In this section I provide a brief discussion of concept pluralism, and draw on that to deliver a general procedure for establishing pluralism as a philosophical position. Concept pluralism has been advanced recently in the philosophy of science literature: it has been suggested about gene (see e.g., Griffiths and Stotz 2013), species (Ereshefsky 1992, 1998; Kitcher 1984), homology (Brigandt 2002, 2003a; Griffiths 2006), planet (Brusse 2016), and biodiversity (Colyvan et al. 2009; Maclaurin and Sterelny 2008; Sarkar 2014), as well as other scientific concepts, sparking both renewed interest in the philosophical analysis of these concepts, and a fruitful methodology for individuating and integrating equivocal scientific concepts. I will use a simplified account of species pluralism in order to illustrate the idea.

There is more than one way of defining "species". Here are three. The phenetic definition classifies species in terms of similarity (usually, morphological similarity). The phylogenetic definition distinguishes species by their ancestral lineages. The biological definition groups species in terms of potential interbreeding.

These species concepts are non-equivalent: each map onto a different "carving up" of the world; they deliver different criteria regarding what it takes for distinct organisms to be part of the same "species". Yet a species pluralist might claim that it may be right to distinguish lions from tigers qua species in some contexts (that the two are morphologically distinct enough for the research task at hand, or have diverged adequately from a last common ancestor, might justify such a move), yet she might also claim that in other contexts it may be right to lump them together for research purposes (that lions and tigers are known to breed in captivity, and can produce fertile offspring, might justify that move). And there may be other useful concepts of species too. ${ }^{20}$ Nonetheless these three species concepts are clearly suitable given the interests of different scientific investigations. Palaeobiologists researching broad macro-level evolutionary patterns might reach for the phenetic concept (for instance), while biodiversity and conservation theorists might reach for the phylogenetic concept, and ethologists or geneticists researching contemporary lineages might reach for the biological concept. Neither concept proffers a unifying analysis

\footnotetext{
${ }^{20}$ It could be argued that this non-equivalence is erroneous or illusory, of course-that some other concept might do all the work that species theorists want it to do, or near enough. Engineering such a concept is a task for the species monist; the ball is in their court.
} 
of species that meets the needs of various scientific researchers; yet the scientific concerns of those researchers are equally legitimate. ${ }^{21}$

If a pluralistic diagnosis of species is on the right track, then insisting that one or another species concept takes precedence-or worse still, demanding that one ought to be adopted unilaterally—is plausibly not justified. Conceptual pluralism, then, is backed by two theses:

The non-equivalence thesis: there is more than one concept of category $\mathrm{C}$ on the table, these C-concepts divide up aspects of the world in different ways, and they cannot be successfully unified.

The legitimacy thesis: these different C-concepts reflect and play ineliminable roles in distinct yet genuine investigations or contexts.

Moreover, pluralism occasions a methodology for elucidating scientific concepts. Investigation utilising the different concepts is divided up among the relevant researchers, and their findings are integrated into more holistic and complementary models or systems that illuminate the pluralistic category at hand. Divide, integrate, conquer.

One might be tempted to think that conceptual pluralism about some category entails eliminativism, but this is not the case (see Brigandt 2003b). A possible conclusion from the simplified account of species pluralism given above is that there are no species, but rather phenetic-species, phylogenetic-species and biological-species. But eliminativism is not necessitated by pluralism. First, some philosophers have argued that although we ought to be conceptual pluralists about (for instance) species and homology, they survive as general categories both for historical exposition and as general targets of explanation. ${ }^{22}$ I will, at least implicitly, follow suit, for music. Second, note that if the message of pluralism is eliminativism, it is eliminativism of an atypical form. Moral error theorists like Richard Joyce (2005) are not eliminativists about moral facts because they think there are distinct, but

\footnotetext{
${ }^{21}$ The phenetic species concept has received a bad rap from philosophers and scientists. For a recent defence of the concept, as part of a pluralistic species framework, see Lewens (2012).

${ }^{22}$ See Griffiths (2006) for homology; Brigandt (2003b) for species. Although see Ereshefsky (1998) for eliminativism about species; Santana (2014) for eliminativism about biodiversity.
} 
non-equivalent, equally deserving contenders for moral facts, but because they think there is nothing like moral facts.

Currie (2016a) argues that we can vindicate realism about categories that are amenable to conceptual pluralism if there are legitimate research programmes that are indifferent to the otherwise legitimate, non-equivalent concepts. It is plausible that music is a prime candidate for such vindication, however this possibility is not explored in this chapter: this would be one goal of the research programme I outline in §4.3. So, admittedly, there is a tricky relationship between conceptual pluralism and (forms of) eliminativism, and in this chapter I will be mostly silent about whether music pluralism leads to eliminativism about the general music category (although I will assume it does not, at least until the argument that it does is put forward), as this concern is not central to my focus on music concepts and my argument for conceptual pluralism.

So, in the following, I seek to establish conceptual pluralism about music. In my view, there are non-equivalent, genuine music concepts, and these are ineliminable parts of different, equally legitimate, investigations. And distinct music concepts are called for in the context of understanding the plurality of equally legitimate, diverse musical traditions at different times and places (recall the points that McKeown-Green and the ethnomusicologists make). To motivate non-equivalence I will consider the leading attempts to provide a unitary account, and then I shift to a discussion of legitimacy.

\section{Conceptual analyses of music}

Unitary accounts of music have been defended by various theorists, each account with its own shortcomings. At the very least, my reasons for thinking that these accounts fail is just as much reason to think that non-equivalence is plausible as it is to hold out for an adequately formulated unitary account. This justifies taking the possibility of conceptual pluralism about music seriously. So I begin by critiquing several leading analyses of music as unitary music concepts.

I do not pretend that I have the final word on unitary accounts of music: needless to say, I can only survey so many analyses. However, I provide strong inductive grounds for thinking that any such analysis will ultimately fail. Moreover, I provide positive reasons to 
endorse music pluralism. In $\S 4.3$ I argue that pluralism underwrites a fruitful research programme in aesthetics, and in the following chapter, a fruitful analysis of the scientific investigation of music.

\subsection{Reductive analyses of music}

Theorists have offered conceptual analyses of music in one of two veins: biconditional reductive conceptual analyses which attempt to capture music via necessary and sufficient conditions, or a multidimensional analysis that aims to capture music's salient features and deliver a method for distinguishing central paradigmatic cases from more marginal cases. ${ }^{23}$

Here I consider and critique three leading contemporary reductive analyses of music. (I then move onto the multidimensional approach in \$3.2.) These are all intrinsic conceptions of music, on my delineation of that terminology, aiming to hook onto similarities rather than institutions/traditions. ${ }^{24}$ Since my aim is to establish pluralism, my criticism of these accounts should be thought of as critique of them as unitary; as such, my criticism will be by way of counterexample. Although this approach involves some appeal to intuitions, note that my goal is to show that a single musical concept cannot satisfy the requirements of an array of different interests or practices. On the plausible assumption that appeals to intuition track one or more of these various contexts, they serve my aim.

\footnotetext{
${ }^{23} \mathrm{It}$ might be noted that a common approach is to provide an operational definition of music, appearing in pragmatic/scientific contexts. But because operational definitions are not intended to be unitary analyses, I will not be concerned with them here (although I return to them later). An additional possibility, not yet applied to analyses of music as far as I am aware, although recently advocated in the general context of art, is that of a cluster concept: a decidedly messy set of desiderata. The idea is that sub-sets of these desiderata, when satisfied, are sufficient for the concept's application (e.g., Gaut 2000). Stephen Davies (2004) has argued persuasively that cluster concept accounts reduce to standard, albeit disjunctive, conceptual analyses. So even with the lack of cluster accounts of music aside, I suspect that attempts to cash out cluster conditions for satisfying a (unitary) concept of music will fail, either due to counterexample, ad hocness, or bloated complexity - that is, the same as with the standard reductive analyses. All this motivates the idea that there are multiple, non-equivalent concepts of music (that is, pluralism).

${ }^{24}$ Relational conceptions of music have not been recently defended as unitary accounts, as far as I am aware, and so are not discussed here. However, unitary relational conceptions of art have been popular (i.e., the institutional theory of art), so a music version presumably would be possible, although of course it would face very similar objections to the art version. Suffice to say, the ball is in the monist's court to defend a unitary relational account of music simpliciter.
} 
Irving Godt has recently advocated the following account of music, calling it a "practical" definition of music:

(2) Music is humanly organised sound, (3) organised with intent (4) into a recognisable aesthetic entity (5) as a musical communication ${ }^{25}(6)$ directed from a maker (7) to a known or unforeseen listener, (8) publicly through the medium of a performer, (9) or privately by a performer as listener. (Godt 2005, p. 84.)

De facto, these eight conditions comprise Godt's analysis of music. (I set aside Godt's first condition, '(1) Unwanted sound is noise', and I discuss his tenth and final-that all human societies make music - below.) According to this analysis, for something to be music it must be an intentionally organised act of communication, self-reflexive or otherwise, by a human or humans, through sound. Thus Godt's concept is normative, in a sense, rather than descriptive: it aims to prescribe the bench-mark for music-status. Godt is evidently concerned with articulating a concept that encourages critique of music. This concept might be legitimate for Godt's evaluative purposes—although I am sceptical, given that it is explicitly skewed towards Western music. The crucial point here is that it fails as a unitary account of music simpliciter.

First, it is sorely narrow. Godt does not take aleatoric music, for instance, to count as "music", and he condemns John Cage's chance-based music. ${ }^{26}$ (Consider performances of Cage's Variations IV and Imaginary Landscape no.4-Godt does not consider these to be music.)

Perhaps more importantly, despite Godt's admission that his analysis 'embodies a deliberate bias toward the music of Western European culture' (Godt 2005, p. 88), Godt also claims that 'There are no human societies without music' (Godt 2005, p. 87)-a mistake, surely, if Godt's own definition is supposed to apply to the term "music" in that sentence.

\footnotetext{
${ }^{25}$ This condition seems to make the analysis circular, but I will spell out what Godt means by "musical communication" during my critique.

${ }^{26}$ Aleatoric music comprises elements determined by chance during its composition or determined variably by performers (for example, in a kind of "choose your own adventure" style) across performances.
} 
Here, developmental and ethnomusicological investigations of music call into question Godt's analysis. Much of the world's music is not intentionally organised into a clear, recognisable "aesthetic entity", for instance; that is, it is not intended to be an object of aesthetic value, or an object to be perceived or evaluated aesthetically. ${ }^{27}$ Lullaby might be one ubiquitous example of this. Lullaby is not usually intentionally organised into an aesthetic entity for aesthetic evaluation, or for strictly communicative purposes, but used to produce changes in affect and arousal in infants, and for caregivers to feel a bond or connection with infants and young children (see e.g., Trehub 2003; Trehub and Trainor 1998).

Ethnomusicological research often reveals that music is entwined with dance, ritual, or both —or indeed other aspects of social life (such as hunting) —and that it is often not intended to communicate, but rather be affective. Several languages do not contain a synonym for the English word "music", but rather "music-dance" (see e.g., Titon 1996); in these societies, movement is ineliminable, requisite for the concept's application. The Swahili people of East Africa, for example, speak of "ngoma", meaning both music and dance which are inextricably connected (Rosman and Rubel 2004). Traditionally, for foragers such as the Blackfoot and Sioux tribes of the North American Plains, music is a communal affair, contributing to ritual and other contexts emotionally (McAllester 1996; Morley 2013). Although the makers of an instance of Blackfoot or Sioux music might also be the only listeners - consider a communal, whole-group music context where everyone is both making and listening; that is, there is no passive audience-it is not best conceived as a "private experience", undermining Godt's distinction.

Second, Godt's analysis is too broad. On it, spoken poetry counts as music simpliciter, as by "musical communication" Godt means 'a controlled sequence of sounds with a definable and ultimately perceivable grammar' (Godt 2005, p. 86). And Godt's analysis may apply to other forms of language use too, including Morse code.

\footnotetext{
${ }^{27}$ Consider Hirsch's view with regards to literature: 'definitions of literature in our time have tended to be oriented to the aesthetic as a guiding assumption of the definition. And to regard literature as primarily and essentially aesthetic is not only a mistake; it is also a very unfortunate narrowing of our responses to literature, and our perceptions of its breadth and possibilities' (Hirsch 1978, pp. 28-29). The same is applicable to the music category.
} 
Importantly, it is that Godt fails to provide a reductive, unitary analysis of music that concerns me. It might be the case that there are some contexts where, for instance, only counting humanly organised, intentional, sound-based communicative aesthetic objects as "music" is the right thing to do, or has practical value. And indeed there might be contexts where spoken poetry or Morse code ought to be included in that grouping. The possibility of this is compatible with my pluralism. Spoken poetry and music, for instance, are often combined in researching Ancient Greek music (Anderson 1994) and the music of some nonWestern peoples, for instance the Kaluli (Feld 1982) and Australian Aborigines (Stubington 2007). And in another context, it might be right to group (say) human, bird, and whale music together (or some other relevant combination of animal musics) for the purpose of researching evolutionary convergences (for example), rejecting music as a necessarily humanly-organised phenomenon, for the research task at hand. This same point mutatis mutandis will go for Levinson's and Kania's analyses of music, discussed below.

Notice how my objections to Godt appeal to explanatory contexts that diverge from that of Godt. Godt is interested in a music concept that is useful for musicological and aesthetic critique. Ethnomusicologists, though, are interested in the socio-cultural roles that music plays; developmental biologists and psychologists are interested in the roles that music plays in ontogeny and parent-infant bonds, and so on. In my view, it is not so much an error that Godt has failed to provide a unitary definition that encompasses some nonWestern cases of music: ethnomusicologists simply require a different concept of music for their project than Godt requires for his. So, appealing to conceptual pluralism about music explains away these clashes.

Turning now to Levinson's analysis of music:

Sounds temporally organised by a person for the purpose of enriching or intensifying experience through active engagement (e.g., listening, dancing, performing) with the sounds regarded primarily, or in significant measure, as sounds. (Levinson 1990, p. 273.) 
According to this analysis, for something to be music it must be organised, in time, towards a particular intent: increasing an experience's value through its auditory features.

Like Godt's, as a unitary analysis, Levinson's is both too broad and too narrow. According to Levinson's analysis, spoken nonsense poetry would count as music simpliciter, for example. And background music at a function might not be attended to, nor might it enrich or intensify the experience of any participants, yet intuitively it is nonetheless music—see, for example, Davies (2012a) on Tafelmusik. Warm-up scales and muzak may also constitute music, albeit poor or base music. Yet these are not typically intended for experiential enrichment, at least in the requisite sense, either. And perhaps nor are some examples of film, video game, and television music (see e.g., Davies 2012a). Moreover, as Kania has noted, a person might:

... sneak into a friend's bedroom and play the opening of the first-violin part of Strauss's Don Juan to startle him awake, with no intention that either of us attend to or engage with these sounds at all, let alone for the purpose of enriching or intensifying our experiences. (Kania 2011, p. 10.)

Indeed, clock radios do exactly this. The alarm—which someone might set purely for the purpose of being woken up, with no intention to otherwise engage with the sounds and so on-is simply the device turning on the music broadcasted by some pre-selected radio station. And similarly, although the actual occurrence of it may be unlikely, a sleepwalker picking up a guitar and filling her bedroom with the sounds of an unintentional rendering of, say, Anthony Ritchie's Melancholia intuitively counts as music, yet does not have the requisite intent.

So as with Godt's analysis, the crucial point is that Levinson does not provide a plausible unitary account. 
Finally, I consider Kania's analysis of music:

... music (1) is any event intentionally produced or organised (2) to be heard, and (3) either (a) to have some basic musical feature, such as pitch or rhythm, or (b) to be listened to for such features. (Kania 2011, p. 12.)

This account is promising. The disjunctive condition permits much avant-garde or experimental music — music that lacks standard features, such as discernible pitches or rhythms. It also allows for a distinction between music and non-musical sound-based art forms (Foley tracks, perhaps): the latter are not intended to be attended to for rhythm, pitch, and the like.

Nonetheless, Kania's analysis is too broad. Spoken-word poetry audiences do listen for meter, rhythm, pitch contour, and so on (think of Allen Ginsberg and the beat poets). Given that spoken poetry is intentionally produced/organised to be heard, and that it has basic musical features such as beat/rhythm, Kania's analysis counts it as music simpliciter. Moreover, Davies (2012a) argues persuasively that Morse code also counts as music on Kania's analysis, given that it is listened to (and systematically organised) with regards to rhythm..$^{28}$ Acts of rhetoric—Churchill's speeches, for instance-are surely events intentionally organised to be heard, which are surely attended to (and performed), at least sometimes, for their musical features (cadence, repetition, assonance, and so on), let alone their emotional affect. ${ }^{29}$ Yet it seems a mistake to count these as music simpliciter, as Kania's analysis does.

\footnotetext{
${ }^{28}$ Davies lists further possible counterexamples: 'Sirens, the chord that sounds when I start my Macintosh, the drumming of my fingers on the desk as I contemplate what to write next, the wind chimes that hang above my porch, and tone languages satisfy the definition without being music (though they may be musical)' (Davies 2012a, pp. 538-539).

${ }^{29}$ Consider, for example, Winston Churchill's June $4^{\text {th }} 1940$ address to the House of Commons: 'We shall go on to the end, we shall fight in France, we shall fight on the seas and oceans, we shall fight with growing confidence and growing strength in the air, we shall defend our Island, whatever the cost may be, we shall fight on the beaches, we shall fight on the landing grounds, we shall fight in the fields and in the streets, we shall fight in the hills; we shall never surrender' (Margulis 2014, p. 162).
} 
Kania's analysis is also too narrow. His first condition excludes the unintentional rendering of Melancholia discussed above, for example. My intuitions, at least, have me take issue with this.

The second condition excludes any example that is not intended to be heard, and in my view, this too is problematic (for example, think of a deaf pianist rehearsing alone at the piano). And by focusing on the intention to produce something that is to be heard, Kania's definition misses something very important about a range of examples, such as ritual or ceremonial music, that might not have been produced with the intention to be heard, but to have a certain effect on the participants. These are distinct intentions. Military marches, for example, are principally for evoking comradery and coordinating action. Moreover, think of trance-inducing ritual music in vodouism-the purported spiritual significance and influence of the music may be an indispensable dimension. ${ }^{30}$ It might be necessary to hear the music for it to have the desired effect, yet it may not have been intentionally produced or organised to be heard. ${ }^{31}$

Time to take stock. What is there to say, more generally, about conceptual analyses of music? In my view, several-perhaps irreconcilable-tensions get in the way of attempts to formulate a unitary music concept. Here are a few:

(1) Intention. Undeniably, intention plays a significant role in music. It is tempting to think that music is (non-linguistic) intentionally organised sound. But not all intentionally organised sound is music (Morse code, for instance) and not all music is intentionally organised (Cage's chance-based compositions; the sleepwalking guitarist). And there are, it seems, degrees of intentional organisation requiring explication. Improvised blues jamming, in which spontaneous response of all players is the point of the music, is organised in real-time by intentional agents, but the music may not have been organised or planned as an overall whole, so its

\footnotetext{
${ }^{30}$ Music has been used extensively by various groups of people, and sometimes in conjunction with drugs, 'in order to communicate with the spirits, and then return to their senses in order to heal the sick, control animals or change the weather... in one form or another wherever there have been hunter-gatherer societies, from the Native Americans of California, to the Inuit of Alaska, the San of South Africa and the peoples of Inner Mongolia' (Hendy 2013, p. 37).

${ }^{31}$ See McKeown-Green (2014) for an extended critique of Kania's definition of music.
} 
intentional organisation may be somewhat looser, say, than a tightly-rehearsed, hierarchically-structured string quartet or central Javanese gamelan performance.

(2) Formal features. Many examples of music can be cashed out in terms of standard musical features such as pitch and rhythm. But, especially in the context of contemporary Western art music, practitioners actively resist the expectation that music is necessarily like that. Many modern examples of music count as music in virtue of their subversion of those features. (Think of Walton's notion of "contrastandard properties" in the general context of art-see Walton 1970.) Obviously, the intrinsic and relational conceptions of music come apart here.

(3) Social role. Music bears out a variety of roles, sometimes conflicting, in human social life: from specialist instrumentalists performing for elite audiences in various highbrow contexts, to facilitating social cohesion through community music-making or ritual/ceremonies, to encouraging discord, anxiety, and political activism, to straightforward personal entertainment.

Two upshots follow from this. First, if tensions such as these cannot be reconciled, then non-equivalence is true. Giving up on monism and admitting a plurality of music concepts would likely see such tensions resolve, as the different features identified here are more or less important for different investigative projects. Second, even if some unitary analysis is ultimately obtainable, it would be so bloated with caveats, epicycles, and so on, that its capacity to do any practical conceptual work would be undermined; alternatively, it would necessitate ad hoc decisions about what counts as music, and what does not. As practitioners continue to flout musical conventions, and as music carries on taking unanticipated turns in its dynamic career (including perhaps its decoupling from musical traditions/practices), any unitary analysis advanced today could not be expected to hold up in the future-in other words, it cannot be assumed that analysing music is like analysing a "static" concept, perhaps causation or time.

\subsection{Multidimensional conceptualisations of music}

An alternative means of attempting to provide a unitary account involves conceptualising music along several dimensions, coming to a kind of "family resemblance" analysis by 
focusing on salient features. This approach is promising. I think that it is worth pausing here to discuss an example of this approach, and discuss my rejection of any plausible version of it as being unitary.

Andy Hamilton allows for music's elusiveness, but nevertheless argues that he can systematise "salient features" of it that "can be elucidated by looking at three different directions of characterisation: acoustic, aesthetic and acousmatic' (Hamilton 2007, p. 46). Hamilton's "directions", can be thought of as constituting necessary conditions that can be realised to lesser or greater extents. (However, these directions are not intended to be jointly sufficient - much non-music will also be captured by taking this approach.) It is useful to represent these planes using a three-dimensional concept-space:

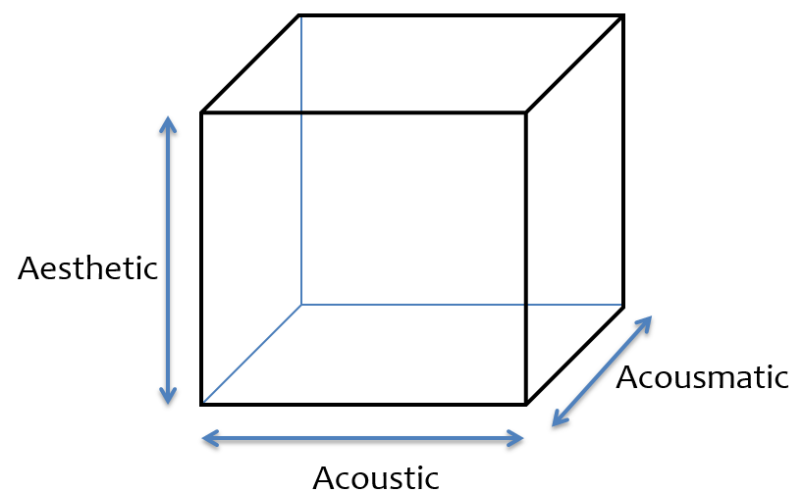

Figure 3.1: Hamilton's music concept-space

The acoustic dimension targets standard musical features such as pitch and rhythm. Accordingly, instances of music that comprise a high preponderance of tones and discernible rhythms (big-band jazz, Beethoven sonatas, say) are placed towards one end of the acoustic continuum. Towards the other end of the continuum - the less paradigmatically "musical” end-would be instance of the noise-based instrumental music associated with composers like Helmut Lachenmann or Karlheinz Stockhausen. Other music, for example the unpitched though highly rhythmic percussion music found in several non-Western societies, would be somewhere in-between. 
So on Hamilton's acoustic continuum we can pick out what counts as music according to a certain criterion-standard musical features-and we can order instances of music according to the prevalence of those features. If something is short on pitches and discernible rhythms, it does not mean that it must be excluded as music-however, it would not be a paradigmatic instance of music.32

Just as Hamilton's acoustic dimension picks out and orders instances of music according to their standard musical features, his aesthetic dimension captures aesthetic features (that is, the properties of music accessed through aesthetic attention and evaluation: examples might include gracefulness, garishness, evocativeness, sluggishness, vividness, and so on). And his acousmatic dimension picks out features of 'the listener's experience or response to sounds as abstracted from their worldly cause', specifically, 'detached from the circumstances of their production' (Hamilton 2007, p. 58). These might include, say, perceptual qualities of the sound emitted by an instrument and subsequently shaped by the acoustic properties of the performance space, though decoupled from the sound's status as a sound-forged-in-that-manner; that is, features of sounds simply "as sounds".

We could, in theory, order instances of music along each of the three dimensions, given their acoustic, aesthetic, and acousmatic properties, and thus plot them. The paradigmatic cases of music for Hamilton-music rich in acoustic, aesthetic, and acousmatic features-will all be grouped together in one corner of the conceptual space.

First off, Hamilton's account is too narrow. For example, Davies (2009) argues that party-goers' renderings of “Happy Birthday" are clear, uncontroversial instances of music, but are rarely-if ever-aesthetically appreciated or evaluated. Yet perhaps they ought to be paradigmatic instances of music. Devoting one dimension to aesthetics clearly skews the space towards "art" music. This raises the question: what legitimises the dimensions that Hamilton appeals to?

\footnotetext{
${ }^{32}$ Hamilton explicitly distinguishes music from sound art. He cites the creative output of practitioners such as Bill Fontana (whose artworks include the recording and mixing of traffic sounds at Brooklyn Bridge) and Alan Lamb (whose artworks include the recording of Australian telegraph wire humming) as examples of non-music sound art.
} 
Note that Hamilton's aesthetic and acousmatic dimensions might also exclude much music historically, prehistorically, and in contemporary non-Western settings. Historically a "bleating calf" song has been utilised by the Blackfoot and Sioux people in a distinctive musical-hunting strategy. The imitative song is performed in order to attract a herd to the top of a cliff face, and then the buffalo are startled and forced over the edge by concealed hunters-in-waiting (see Kehoe 1999; Howard 1984). It remains to be seen that taking this song out of its wider social context and appreciating it merely as song-for its aesthetic and acousmatic properties—should make it more a case of music.

Second, as already noted, instances of non-music are captured by this approach too. This leads to the "overextended family" problem with such "family resemblance"-type strategies (Fitzgerald 2000). And although paradigm cases of music, by Hamilton's lights, can be distinguished on his model, there is no systematic way of distinguishing the less paradigmatic yet genuine family members from non-members. The problem is that we are conspicuously lacking precise criteria of family membership—even on a relational conception of music, where to draw lines is very fuzzy — so as an approach to unitary analysis, this strategy looks problematic (see Dawes and Maclaurin 2013 for similar points in the context of analysing religion). However, if we give up on the idea of "unified" family resemblance for music concepts, a non-essentialist family resemblance approach is compatible with pluralism. The bottom line is that once we realise that the different dimensions have different importance in different contexts, Hamilton's approach is, plausibly, best and most charitably re-conceived as a version of pluralism.

So although, in my view, "dimensional" approaches to analysing music are interesting and potentially useful, I deny that there would be a "best" unitary multidimensional account. For it to have a claim to plausibility, either such a conceptual space must comprise so many dimensions lest music be skewed towards particular notions (bringing in, for example, social role, degrees of intention, and so on) as to become, in practice, unwieldy. (And, in doing so, encapsulating a horrifically bloated overextended family.) The alternative is that the admissibility/legitimacy of the dimensions-the planes or slices through that conceptual hypercube that different theorists will target—would depend upon the explanatory interests of the theorist positing the concept: in other words, suggesting pluralism and eschewing the search for a unitary concept. 
For the anthropologist, music's integration and roles within human sociality might be an ineliminable musical dimension; for the structure-focused musicologist, such a dimension might prove to be a superfluous nuisance. Furthermore, unless there are robust, systematic connections between dimensions like Hamilton's, researchers who simply do not care about the aesthetic aspect of music have no reason to include that dimension. A composer/producer of jingles might care about her music's psychological and emotional affect, but reject her work as "art" or as something to be considered aesthetically. Parents singing lullabies, or particular rhythms played for ritual purposes, background music/Tafelmusik, and instances of "Happy Birthday" are not "music" any less for the reason that they lack an aesthetic angle.

Moreover, if pluralism is right, it undermines Hamilton's methodology—prima facie there is no reason to think that the multiple music concepts identified by a pluralistic programme could not be analysed in the standard reductive way, giving rise to more precise operational definitions and distinctions for research purposes.

\section{A plurality of music concepts}

I take the preceding section to have shown that music is elusive and difficult to define. This can be easily explained: there is not one music concept. Rather, there are multiple music concepts, useful for different contexts.

In this section I outline two guises of my pluralism, and I distinguish my view of music's plurality from Nercessian's. I then reply to the objection that music really is a unified phenomenon. Finally, I argue that there is a positive reason to endorse pluralism-that it grounds a research programme in aesthetics. I then go on to provide a discussion of pluralism's usefulness in the scientific study of music in the following chapter.

\subsection{Two guises of music pluralism}

First, different music concepts can be reached for in different explanatory contexts. For instance, an anthropologist could analyse "Happy Birthday" as an ethnographic object, while a musicologist might analyse that same song in terms of its music theoretic structure. Yet conceived thusly, "Happy Birthday" qua ethnographic object, and "Happy Birthday" qua auditory form represent distinct targets of two investigative interests, methods or 
agendas. The properties and roles played by those properties that are investigated by these researchers will be very different.

Second, music concepts can classify different phenomena together as music, and different concepts will distinguish music from non-music in different ways. As we have seen, some theorists analyse, or explicitly privilege, a Western characterisation of music (think of Godt's analysis discussed above). Yet other societies have distinct concepts of music. Some of these concepts might disregard any recognisable "aesthetic" dimension, or might require including ritual or dance, for instance. Some cultures lack a word for music, yet they clearly make that which Westerners would consider music. Sometimes what we in the West consider intuitively to be examples of music from a non-Western tradition are not considered music according to the local music concept. Drawing on the research of Katherine Hoffman, Arbib reports that 'the chant of a muezzin is not considered by Muslims to be music, and attempts to meld such chant with explicitly musical forms are considered deeply offensive by some, but a welcome innovation by others. Here we may contrast Gregorian chant, which is certainly considered a musical form' (Arbib 2013, p. 19). (This situation plausibly represents the tension between a Western intrinsic conception of music and non-Western relational conceptions of music.)

And music pluralism seems to be espoused by some non-Western cultures. Some cultures have different words for separate music concepts. Hiromi Lorraine Sakata, a researcher of Afghanistan's music, distinguishes ghinā from musiqi. On the plausible assumption that this distinction is useful, it undermines the (Western academic) music monist's approach. Moreover, she says: 'It is clear that the concept of music that exists in Afghanistan does not parallel the Western notion of music' (Sakata 1983, p. 74)

So there are two guises of my conceptual pluralism: pluralism pertaining to research targets (i.e., different music concepts are legitimised by the different research programmes that target music, à la species), and pluralism across phenomena/systems (i.e., different music concepts are required for the diversity of the world's musics). Understanding how the music category is realised/instantiated in practice - that is, understanding, crossculturally, what it is for a phenomenon to be "music"-requires analysis of different concepts due to the variability of music's manifestation. 
The first guise recognises that there is a plurality of music concepts. So as it plausibly is with categories like species, gene, and so on, researchers analyse music differently, going by the needs and purposes of the inquiry. (Researchers can and do provide operational definitions; some actual examples of operationalisations offered by researchers are discussed in the next chapter.) Music cognition researchers, for instance, have different explanatory targets to anthropologists, for instance-and in some cases, even to each other. So as a result, researchers might approach the same target of study differently.

Consider the target of music's investigation as art object. The unitary conception of music analysed by Godt, Levinson, and Kania centres on performed music which is heard. But is the score-reading, Western art music fanatic, revelling in Mahler's $2^{\text {nd }}$ Symphonyconjuring up the music in her mind's ear, through reading a score-copy-not appreciating music? Could she not aesthetically evaluate the object of her attention (that is, sound qua auditory image/mental representation) as someone listening to a performance or the playback of a recording also could? Perhaps a workable concept of music for aestheticians will focus on the aesthetic properties of music accessible for evaluation from multiple modes of experience, including through mental imagery, rather than squarely on performed music. Zangwill (2014), for instance, has recently advocated a non-reductive notion of music via "musical beauty". Such a concept could well be plugged into aesthetic theories and put to good use. (Of course, the utility of Zangwill's particular notion might remain to be seen.)

An anthropologist's or ethnomusicologist's music concept, on the other hand, might explicitly eschew aesthetics and the notion of "art object". The performance features of an instance of music might be central to an ethnomusicologist's music concept. An evolutionary theorist's music concept might centre on the product of particular human behaviours and capabilities, the traits that underlie these, and the interaction of genetics and environment in their regard. In short, different music concepts are useful for different investigations, and grounded by legitimate concerns. Moreover, the different music concepts of researchers may complement one another; I elaborate this point in the next chapter. 
The second guise recognises the plurality of phenomena conceptualised as music by different peoples (e.g., music-dance, ghinā/musiqi, Western classical/folk/popular music, experimental noise music, and so on). The investigation of aspects of one music system might carry explanatory power when applied to another, yet cross-system applications cannot be simply assumed. There is significant variability. Analysing central Javanese gamelan music in terms of objective pitch, for example, would obviously be folly. ${ }^{33}$ Yet not so in the context of analysing Western art music: there is an interesting historic story to tell about equal temperament and the standardisation of $A=440 \mathrm{~Hz}$ (see e.g., Duffin 2007).

Both guises of pluralism are important. Together they frame the phenomena (the explananda) and the concepts utilised. There is no unitary music concept; no fact of the matter as to the extension of "music", on some occasion of use, divorced from some music concept. This has implications for philosophical debate.

Given pluralism, it is useless to ask whether something is music simpliciter. There is debate in aesthetics (see Davies 2003; Kania 2010; Levinson 1990) about performances of John Cage's notorious "silent" composition, 4'33": are they music? In my view, this is the wrong question to ask. Since I am a pluralist, I think the question ought to be "are they captured by the relevant music concept at hand?", or "which music concepts capture them?". 34 Performances of 4'33" might ineliminably count as music for the purposes of one theorist's research—perhaps, research into the role of concert piano performance practice, or the history of twentieth century American concert performances (where a relational conception of music might be at hand) - and not on another's. For those coming from an ethnomusicological perspective or the perspective of a non-Western musical practice, or from a historic slice of the Western art musical tradition, it may seem inconceivable that performances of 4'33" should count as music. And for biologists and psychologists concerned with human musical capacities, whether 4'33" counts as music or not is a largely irrelevant, tangential issue.

\footnotetext{
${ }^{33}$ Although gamelan musical scales have a loose conventional structure, gamelan-makers have their own distinct tuning systems, so there are intervallic discrepancies across sets of instruments. Thus the same traditional melody will sound different played on different sets of instruments-not only transposed, but internally (i.e., the relations between the pitches that make up the scale) transformed.

${ }^{34}$ And perhaps which non-music concepts capture them too (e.g., Davies' theatrical performance concept).
} 
As I argued is the case with "sound" in the previous chapter, the situation with “what is music?" questions results in a Chalmers-style deflationism (see Chalmers 2011). Of course, this is not to deny that there are substantial disputes about music concepts. If theorists using the same music concept disagree about the music status of performances of 4'33", say, then the disagreement is not merely verbal: the theorists will need to resolve the dispute by reflecting on the concept in question and the performances in question. The different music concepts utilised by different theorists target different properties and will be shaped by the different roles they play in investigation.

Andy Nercessian (2007) has, perhaps, come close to touching on my position. But it is not pluralism, as I see it. So it is worth outlining his self-styled "pluralistic" view, and clarifying where I depart from it and why. Like me, Nercessian argues that music has 'many guises' (Nercessian 2007, p. 24). His method is to distinguish three "musics"—classical, popular, and folk. ("Classical" is not supposed to reduce to "Western art music", but is intended to also capture Indian, Asian, and Middle Eastern art-musical traditions, and the like.) Nercessian argues that, for the most part, it is uncontroversial whether something is an instance of classical, popular, or folk music. However, he also notes that there will be cases that overlap, blurring the boundaries of two, or perhaps all three, categories-and he suggests that any given borderline case might be considered folk music for one person, classical for another; that is, it can depend on the perspective of the person approaching the music. The category music is thus conceived as that of a tripartite Venn diagram:

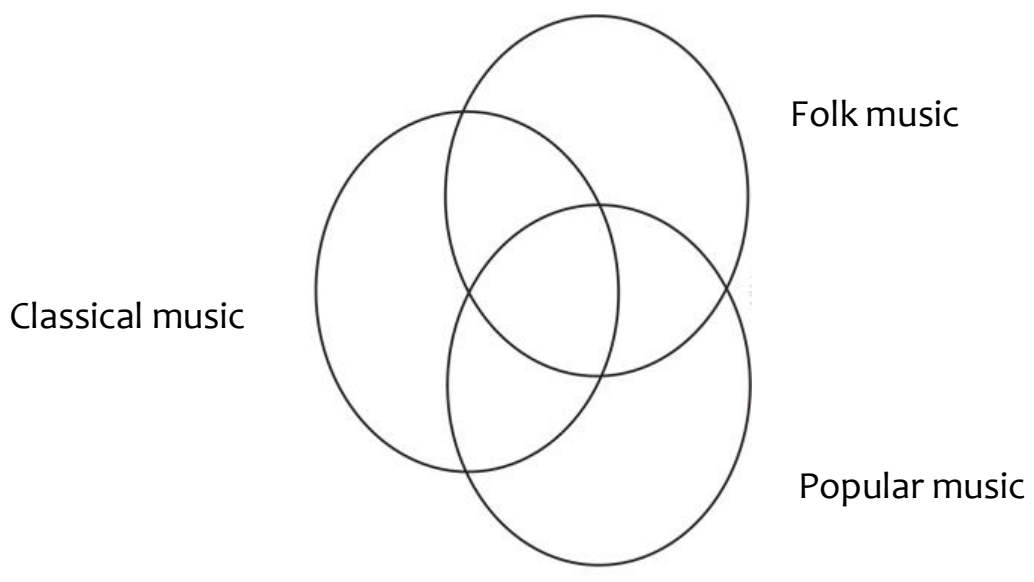

Figure 3.2: Nercessian's music concept-space 
This might look to some like a pluralistic picture - that Nercessian is advocating a plurality of music concepts. However, this is not the case, as Nercessian operates under an everlooming, though tacit, unitary concept of music. De facto, his distinctions are better seen as broad types or genres of some tacit unitary music concept. So I depart from Nercessian's model in several crucial ways.

First, Nercessian does not see the theorist's job as analysing distinct music concepts, but rather 'to discover essential things in each type of music and to use these, and not those elements that are present only in particular tokens of music, in the definition' (Nercessian 2007, p. 25). He thinks that a unitary analysis of music is possible, albeit 'long and disjunctive' (Nercessian 2007, p. 85). Indeed, for Nercessian, the concept of music comprises 'one body of notions that remain together' (Nercessian 2007, p. 265). As already noted, I am sceptical of the usefulness of such a definition of music simpliciter.

Second, Nercessian is analysing only the Western notion of music. This ignores entirely one guise of my pluralism: that there are multiple systems that count as music. Conceptualisations of those systems need to be accounted for too. Nercessian is well aware of this, admittedly. He allows that, in different societies, 'people use their term for music to refer to a different entity to the entity we refer to when we use our word for music... the world is not composed of one music in different forms, but rather many musics which are in essence different' (Nercessian 2007, p. 256). However, these musics are black-boxed from his analysis.

\subsection{But we know it when we hear it!}

There is a straightforward objection to my pluralism: there must be a unified music concept—we just don't know what it is yet—after all, we know it when we hear it!

Stephen Davies writes:

... we can immediately and almost infallibly recognise [music], even when it comes from a culture that is foreign to us. Though I may be unable to predict how such music will continue or to recognise errors, and though it 
may sound strange to me, I can be in no doubt that it is music that I am hearing. (Davies 2012a, p. 535.)

Andrew Kania writes:

... most of us can tell whether, and which of, the sounds we are currently hearing are music. This is so whether or not what we are listening to is a familiar piece, a piece we have not heard before, or even music from a culture or tradition with which we are unfamiliar. (Kania 2011, p. 3.)

However, the ability to recognise music when we hear it does not undermine pluralism. There are two points to be made here.

Given the amount of overlap allowed by legitimate music concepts (just think of the overlap between the intrinsic and relational conceptions of music), it may even be unsurprising that, for very many cases, we can all immediately recognise something as "music". There may well be instances of music that concurrently instantiate many music concepts. So we might be legitimately recognising parts of this overlap as "music" when we hear it and all agree.

Second, in the hard cases we do disagree: as we just saw, there is live debate over whether performances of Cage's notorious 4'33" count as music (simpliciter). And indeed, much contemporary avant-garde art music might not be immediately recognised by the untrained ear as music. Even theorists with a trained ear diverge-recall Godt on Cage's Variation IV and Imaginary Landscape no.4.

Music is undoubtedly a "phenomenon"- it is a category which we recognise and want to explain—but this does not entail that music is unified. We distinguish between different species when we see them, yet why think that scientists must constrain themselves to a single species concept? Recall that pluralism about species concepts is justified by the lack of a unifying concept which meets the needs of various scientists, and the equal legitimacy of those scientific investigations. In my view, the same is true for music. 


\subsection{Towards a philosophy of musics}

Understanding music in its many manifestations requires a diverse array of contributions, from musicologists and philosophers, to anthropologists and ethnomusicologists, sociologists, archaeologists, evolutionary biologists, computer scientists, acousticians, neuroscientists, psychologists, music therapists, and more. That the study of music represents such an interdisciplinary research cluster as this implies pluralism: given the range of explanatory interests, research questions, and lines of evidence relevant, no unitary definition of music will do the job across the board. I explore this point further in the next chapter. While an art historian or aesthetician's concept might take an aesthetic dimension to be ineliminable; an ethnomusicologist might reject this, and take social function as ineliminable, and so on. Ann Buckley, for example, laments the common 'tendency to view the study of music... as primarily motivated by aesthetic concernsshape and style, moral tone-setting, art object, rather than serving as 'functional craft', used to meet social needs of many different kinds' (Buckley 1998, p.12). Neither approach is in principle right or wrong, in my view. Both emphasise different concepts of music, necessary for their associated research agendas.

I take the discussion so far in this chapter to have provided at least inductive reason to think that there is not a unitary concept of music. I have not defended any music concepts in particular, though I have gestured to a large number of potential candidates (from ethnomusicology, aesthetics and art history, psychology/biology, ordinary critique, and so on). This is because my goal in this chapter has been to establish pluralism, but not to argue for the legitimacy of these various contexts. But here, I sketch how that project might proceed. That is, I argue that there is positive reason for philosophers to be pluralists about music: pluralism grounds a research programme in aesthetics. To the extent that this is plausible, it further undermines the "default status" of the standard view that music is a unitary concept.

Consider the following passage:

Music may be what we think it is; it may not be... Music may be that to which some dance or pray or make love; but it's not necessarily the case. In some cultures there are complex categories for thinking about music; in 
others there seems to be no need whatsoever to contemplate music. What music is remains open to question. (Bohlman 1999, p. 17, emphasis mine.)

Conceptual pluralism gives rise to a methodology that is well equipped for tackling Bohlman's challenge. It underwrites a research project in aesthetics: a philosophy of musics. The programme, as I see it, consists of (1) identifying what makes for legitimate and illegitimate concepts of music, (2) identifying different investigations of music, (3) identifying different music concepts and analysing them, (4) relating these concepts to issues in ontology (for instance, can we vindicate realism about the music category in the way Currie 2016a suggests we can for species?), and (5) relating these concepts to theoretic and empirical investigation.

For an example of how this research schema might play out, consider possible treatments of the notion of legitimacy. Participants in the research project could ask: what underwrites the legitimacy of a music concept? Are music concepts grounded on the contestability of claims, or on some other factor? Should intuitive music concepts be grounded in the way that they are? Are pseudoscientific concepts of music legitimate? What are the more general applications of philosophical thought about legitimacy?

Conceptual pluralism also delivers a normative research project: can participants engineer suitable music concepts for different investigations? Despite my pluralism, I do not advocate a conceptual "free-for-all" relativism. ${ }^{35}$ It may be that there is a best music concept for some particular research interest-as is plausibly the case for species. (And there may be failed music concepts-just as there are failed species concepts, think of Aristotelian essentialism about species.) If it turns out that our brains process some of the basic musical features of auditory stimuli within an encapsulated module, then as McKeown-Green (2014) suggests, music with these features might form a special category worth investigating, which psychologists and neuroscientists could conceive of as music. ${ }^{36}$ Anthropologists, on the other hand, might steer clear of such a music concept, utilising one

\footnotetext{
35 See Maconie (1990) for the idea that what counts as music is subjectively determined by each individual.

${ }^{36}$ See McKeown-Green (2014), 403, fn. 23; also Peretz and Coltheart (2003).
} 
that emphasises music's role in the community. A plurality of conceptual analyses will provide the next step towards understanding the pluralistic nature of music. (And, even if I am wrong about pluralism —if a unitary analysis of music is possible —-this research programme will help us get it.)

To get this programme started, consider the following music variants of the four art concepts identified by Mag Uidhir and Magnus (2011, taken from pp. 91-92):

Historical music concept, i.e., music emerging from, belonging to, embedded in, music-historical traditions or narratives—productive for historical inquiries.

Conventional music concept, i.e., music recognised, accepted, targeted, governed by conventions, institutions, and practices-productive for sociological and anthropological inquiries (as well as for legal and economic issues).

Aesthetic music concept, i.e., music satisfying some aesthetic function; for example, affording some aesthetic attitude, experience, interest, valueproductive for value inquiry and certain cognitive inquiries involving perception.

Communicative music concept, i.e., music that acts as a vehicle for the communication of certain contents; for example, representation, semantic, or expressive content-productive for certain cognitive inquiries involving learning and emotions, as well as for moral evaluation.

Clearly there will be much overlap across these four music concepts, but of course they will diverge on the problem cases philosophers have been concerned with (e.g., 4'33"). As Mag Uidhir and Magnus note for art, for music the historical and conventional music concepts will exclude some folk music, "outsider" music, and music in some thought experiments involving possible worlds and aliens from far away galaxies and times; the aesthetic music concept will exclude much contemporary music; the communicative music 
concept will exclude absolute/pure instrumental art music. Yet any of these music concepts may be legitimate for some research or theoretical purpose. And there will be other music concepts too-not all music is art, after all (see e.g., Kivy 1991). A music theoretic conception of music, for example, might target something like sound structures that comprise standard features (rhythm, pitch, etc.), disregarding the concern about whether intention is necessary for the concept's application (Mag Uidhir and Magnus explicitly maintain intention as a necessary criterion for application of all four of their art concepts). Since it is plausible that these extensionally non-equivalent concepts, or something like them, are useful for legitimate investigations, my pluralistic research programme is vindicated. Future research however will tell the extent of this-or indeed whether my contentions ultimately lead us to a monistic concept after all; the proof is in the pudding.

To this end, operational definitions of music, not usually discussed by philosophers, pave a way forward. So before closing I give a brief example, to demonstrate an evaluative aspect of this research programme. Music cognition researchers Honing and Ploeger distinguish music from musicality as follows:

We define musicality as a natural, spontaneously developing trait based on and constrained by our cognitive system, and music as a social and cultural construct based on that very musicality. (Honing and Ploeger 2012, p. 516.)

Assessing this operational definition of music does not require the method of (intuitive) counterexample. Rather, it requires an evaluation of its suitability as a guide for focusing research on the phenomena investigated. So this definition might be undermined if it can be demonstrated that splitting music from musicality along cultural/biological lines is unjustified, perhaps due to the dynamic nature of bio-cultural co-evolution (discussed later in this thesis). Perhaps it could be argued that taking music's social expression into account is required for understanding the evolution of musicality qua cognitive trait. And perhaps music's social expression should be taken into account in researching musicality's developmental emergence. 
If so, then for music cognition research, music and musicality need to be more carefully distinguished than Honing and Ploeger allow: perhaps it is a mistake to define musicality as "spontaneously developing" (which, presumably, is intended to detach musicality from contingent development due to influences of enculturation).

If not, Honing and Ploeger might be justified in their distinction, which allows them to black-box the complexities of the influence of social and cultural forces in evolutionary psychological contexts, permitting a sole focus on musicality qua cognitive trait.

In the end, the success or failure of Honing and Ploeger's operationalisation turns on the work it is supposed to do and whether it achieves that. (I am sceptical; see the following chapter.)

\subsection{Conclusion}

In this chapter I have argued for conceptual pluralism about music. I have critiqued currentstanding analyses of music as suitable unitary conceptions of music simpliciter, and I have motivated the shift to a pluralistic treatment. I have outlined my pluralism's two guises, I have replied to the objection that music is unified because it intuitively seems that way (after all, we can tell it when we hear it!), and I have demonstrated pluralism's utility by outlining a potential research programme in aesthetics and (tentatively) distinguishing several music concepts, based on Mag Uidhir and Magnus's art concepts.

In the next chapter, I turn to the scientific investigation of music itself in order to continue explicating pluralism's value. I argue that music pluralism can drive integration and I provide a conceptual framework for the analysis of music's investigation-an interdisciplinary project comprising disparate research agendas, research questions, and lines of evidence. I argue that the music pluralist is better equipped than the monist to make sense of the research cluster that investigates the science of music. I consider the prospects of a pluralist's perspective generally with reference to particular areas of the study of music, and I close by briefly considering the possible value of pluralism about aesthetic concepts by and large. 


\section{Chapter 4: Music pluralism and scientific investigation}

Music is a fascinating target of scientific inquiry. As well as underwriting a fruitful research programme in aesthetics, my conceptual pluralism has important upshots for the scientific study of music. In my view, pluralism can lead and drive integration in scientific research. To this end, I distinguish complementary from antagonistic relationships between music concepts. Complementary definitions of music can work in tandem; antagonistic definitions give rise to empirical dispute. So, empirical work and operational definitions are not independent: given a particular research question, or approach, some definitions can be more or less appropriate for the job. Determining the best definition for the job at hand can itself be an empirically-informed task. There is a back-and-forth between conceiving of the target of inquiry and investigating that target. In other words, good operationalisations can be legitimate research findings, not necessarily stipulated assumptions.

In this chapter, (\$1) I provide a sketch of the science of music, (\$2) I draw upon several operational definitions of music, and $(\$ 3)$ I discuss the utility of pluralism in music's investigation (rewriting and extending Currie and Killin 2016). I demonstrate throughout the plausibility of music pluralism's use as a promising conceptual framework. This challenges the monist to provide an account of the rich diversity of music research within some monistic framework and to motivate "making do" with a unitary music concept.

\section{A sketch of the science of music}

The scientific study of music is not a single, homogeneous discipline. Musical research is approached from a variety of stances, disciplines, perspectives, and background theories, encompassing a plurality of research questions and lines of evidence. Researchers include, but are not limited to, anthropologists, archaeologists, musicologists, neuroscientists, psychologists, music theorists, music therapists, sociologists, computer scientists, mathematicians, evolutionary biologists, acousticians, and philosophers. So before I launch into discussion of the upshots of pluralism about music for music research, in this section I will embark on a brief detour: I first sketch the kinds of research questions that drive musical science, and second, the evidential sources harnessed by those research projects. 
In order to give musical science a rough and partial image, it is useful to characterise the complexity of research areas along two dimensions, temporal scale and level of organisation/degree of abstraction, common notions in the philosophy of science. Figure 4.1 loosely and partially sketches the research landscape:

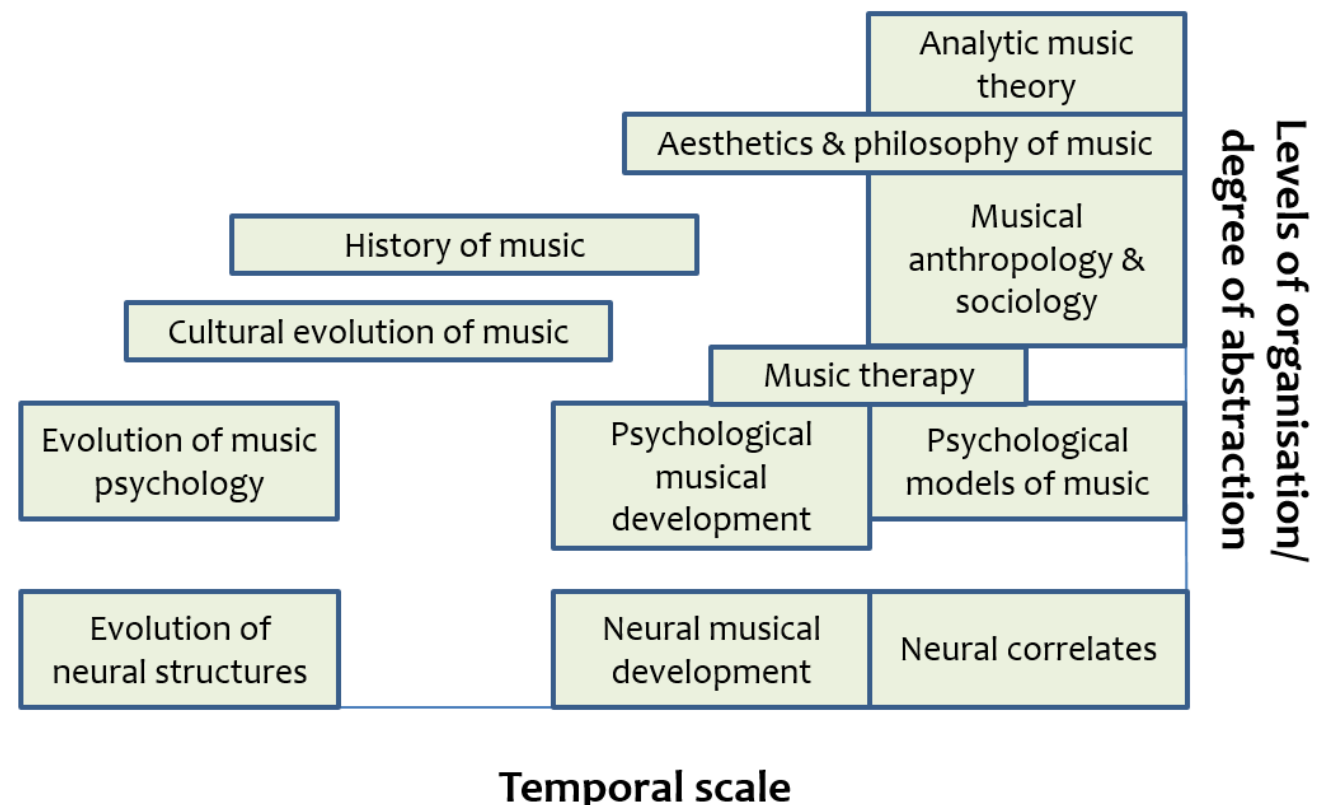

Figure 4.1: Research areas along dimensions of temporal scale and organisation/abstraction

This representation is general, rough, and not exhaustive, and there will be other useful ways of characterising the research space too. I discuss the utility of this characterisation in what follows. Even so, some of the domains here may well be misplaced. As I go on to argue, if the cultural and biological aspects of music evolved dynamically, then artificially separating the evolution of musical psychology and music's cultural evolution may be misguided (even though relevant changes may occur over different time frames). This thought will be returned to in due course. And note that the figure does not clearly distinguish, for example, objects of investigation from research methods: the figure is heuristic, and would require many more dimensions in order to provide an authoritative, thorough characterisation of musical science. 
Consider first temporal scale. Some research questions about music are long-range. They concern glacially-paced processes in the deep past. Others cover relatively shorter time frames and quicker processes. And still others concern the here-and-now.

Ernst Mayr (1961) famously distinguishes proximate from ultimate causes of biological phenomena. Although I do not here commit to a hard-and-fast distinction, it is a useful way of illustrating my point. ${ }^{37}$ According to Mayr, an ultimate cause is called for in explaining the existence of a trait in terms of evolutionary pressures. Some researchers ask, for instance, whether music is an evolved adaptation. A proximate cause is called for in explaining factors that shape an organism over its own lifetime. Some researchers might investigate the age from which children can distinguish melodies or show preferences for music from their own culture from a random sample, for example. To give a further (albeit comparative) example of the rough distinction, consider birdsong research. A proximate explanation of a bird's singing might be that the increasing levels of light in spring triggered a hormonal release in a male songbird's brain (Moore et al. 2006). The ultimate explanation of a bird's singing might be that its song indexes the quality of the male bird. It has evolved to sing in order to attract mates. The songs of bulbuls (Pycnonotidae) 'are carefully arranged to signal strength and fitness' (Bulbulia 2005, p. 86).

So my temporal scale is intended to track something like the phylogeny/ ontogeny continuum (Gould 1977), but is not limited to questions of development and evolution. At the scale's extreme right end, questions are near-atemporal. Much cognitive science and psychology research targets the characterisation of contemporary human minds, not investigating historical forces—for some recent examples, see Eerola (2012) for a computation model of the perception of musical emotion, Neuhaus (2013) for approaches to studying how minds process musical form, and Tesoriero and Rickard (2012) for a discussion of frameworks for music-facilitated memory recall. Moreover, the theoretic analysis of Western musical scores and performances in terms of meter, scale, and so on, is hardly temporally-bound. (Admittedly, musical features associated with music-theoretic concepts are temporally-bound — that is, meter, tonality and the like have particular

\footnotetext{
37 Indeed, such commitment would be unwise (see e.g., Calcott 2013; Laland et al. 2013; Ariew 2003; Beatty 1994).
} 
meanings indexed to different eras in music history-yet a chronology of music-theoretic concepts might fall under "History of music" in Figure 4.1.)

Moving from left to right along the temporal axis thus sees a shift from research questions about music's origin, evolution, and subsequent persistence and development, to questions about how musical cognition develops over an individual lifetime, to theoretic questions about musical structure.

The other axis orders research areas by their level of organisation and/or degree of abstraction. These can be spelled out in terms of levels of scientific description or ontological relationships between investigative targets (see Craver 2005, 2007), however I am only applying general notions of organisation/abstraction here. Think of the rough divide between the material and formal sciences. Roughly speaking, some research questions are relatively less abstract than others: for example, what are the neural correlates of musical behaviour? Other questions are pitched at a higher level of organisation than this: what is the role that music plays in modern societies? And at the more formal/abstract level, researchers might ask: which mathematical structures capture certain musical forms?

A psychologist might posit a mental model in order to explain some musical capacity or behaviour, while a cognitive neuroscientist might posit neural mechanisms that would underwrite such a model (and thereby that same capacity or behaviour). Cognitive science, for example, typically focuses rather narrowly on lower levels of organisation/ abstraction, such as neural and psychological research. Critics urge researchers to include social and cultural considerations more explicitly (Bender et al. 2010; Cross 2012b; Stevens 2012; Trehub et al. 2015), thus arguing for a wider focus. Moreover, theoretic musical features (key, tone, meter, and so on) play an important role in the construction of models and studies of less abstract sciences.

Thus this axis conflates levels of organisation with degrees of abstraction, in order to straightforwardly capture both material and formal sciences along two axes. Indeed, taking the notion of levels of organisation too literally here results in folly: think of the placement of highly theoretic work like music theory-yet human social groups do not themselves come together to form the mathematical structures of music theory. Recall, the 
purpose of this sketch is not to arrive at an authoritative layout of music research, but to motivate pluralism. So this conflation might be problematic if the crucial purpose of this discussion was to deliver a thorough analysis of musical science (rather than show the utility of pluralism). Again, the figure is heuristic. A thorough attempt to capture the landscape would require more dimensions, and that the figure fails to fully characterise the rich diversity of musical research is grist for my mill, demonstrating that more complexity is required to tighten the analysis.

This discussion might hint at reductionist models of scientific explanation, for instance the 'layer-cake' picture of Oppenheim and Putnam (1958). However, as will become clear later, I am not here committing to any reductionist view: indeed recognising levels of organisation is often part of rejecting reductionism. And sketching out research agendas by levels of organisation need not involve an assumed reductive relationship between the levels.

The point is that combining the two axes-temporal scale and level of organisation/degree of abstraction-provides a coarse and partial perspective of possible research angles from which researchers embark on the project of understanding music. Embracing a research cluster represented by this perspective will, I argue, demonstrate pluralism's usefulness in the study of music.

I turn now to lines of evidence. A line of evidence is produced by some scientific technique or technology, scaffolded by background theory that explicates its operation (i.e., "how does it work?”) and underwrites its explanatory usefulness (“what does it prove?"). Lines of evidence in the study of music include, but are not limited to, brain scans, behavioural responses (from various developmental stages-infants, children, adolescents, adults, seniors), archaeological finds, cross-cultural ethnography, cross-species comparative data, psychological data (e.g., happiness/wellbeing), genetics, and more. Perhaps obviously, lines of evidence are best harnessed by the researchers from particular disciplines-psychologists, neuroscientists, archaeologists, biologists, to name a few. And lines of evidence may typically, though not always, correspond with distinct levels of organisation/degrees of abstraction. 
So progress in the study of music is truly an interdisciplinary pursuit: there is a wide range of research angles, and a plurality of evidential sources. In my view, understanding the relationship between the different investigations, and integrating their various findings, is crucial for progress.

Turning to the philosophy of science can help here. Philosophers have discussed the relationship between research questions (or research agendas/perspectives) and lines of evidence, for instance in reference to the study of evolutionary novelties (Brigandt 2010; Love 2008). By these philosopher's lights, different evidential sources can play different roles depending on which research question is salient, and the explanatory significance of some approach varies with the question pursued. This is because some lines of evidence carry the explanatory force for some particular research question while other sources play a supporting role. Pragmatic concerns about scientific explanation allow researchers to determine "explanatory force": given the research question at hand, we can ask which agenda provides a plausible relevant answer and which play supporting roles, providing additional reasons to accept that answer, or at the very least constraining or establishing salient facts that are consistent with that answer. For example, for Brigandt (2010), the explanatory force of accounts of the evolutionary origin of novelties is carried by developmental biology and functional morphology (with hypotheses from that agenda being supported and constrained by research on population and developmental genetics, phylogeny, ecology, and palaeontology). This ‘epistemology of explanatory integration’ (Brigandt 2010, p. 309) is opposed to the reductive unification of Oppenheim and Putnam (1958), whereby research agendas at lower levels of organisation are always more fundamental for the purpose of explanation. Brigandt, Love, and others show this is not always the case. And in my view, this is happily compatible and consistent with conceptual pluralism: if lower levels of organisation were always more fundamental in scientific research, the music monist has a plausible claim to a "bottom level" music concept. Straight off the bat, the music pluralist is better equipped than the monist to account for non-equivalent, though legitimate music concepts salient for research at multiple levels of organisation. The monist needs an argument for motivating the attempt to make do with a unitary music concept across the board; the pluralist does not. 
To give an illustration of this epistemology of explanatory integration in musical science research, consider the discussion of psychological models in Grahn (2012). Grahn's research question is along the lines of the following: which of our psychological features allow us to recognise musical rhythm? She distinguishes interval models from entrainment models. Interval models contrast the pulse of a fixed internal clock (or clocks) to external rhythms; entrainment models fit an internal oscillator to the external stimuli. Grahn questions whether interval models could be fine-grained enough to handle musical rhythm (rhythms being, after all, sequences of time intervals), and she focuses on the role of neuroscientific data in helping to decide between the two models. So the psychological research should be understood as carrying the explanatory force for Grahn's inquiry: in principle we would provide an answer to her research question by claiming that interval models, or entrainment models, or perhaps some other model, if instantiated in human psychology, would allow us to recognise musical rhythm. Neuroscientific data (including functional neuroimaging data and studies of brain-damaged individuals) plays a supporting role by helping us distinguish and constrain psychological hypotheses. Clearly, for any psychological model to be veridical, it must be possible for it to be instantiated in the human brain. (If the model can't be actualised it cannot plausibly carry explanatory force for that aspect of human psychology.)

In reference to Figure 4.1, we can characterise this kind of relationship as one that holds between low-level research (about neural correlates) and slightly more abstract research (about psychological models for music). The psychological models do the relevant explanatory work, carrying the explanatory force; neuroscientific data provides additional empirical support by individuating models and evidentially discriminating between hypotheses (and, plausibly, this does not make it more fundamental, contra Oppenheim/ Putnam reductionism). Note that the relevant music concept for the explanatory level of psychological modelling for this target of inquiry may be indifferent to an historical, institutional, aesthetic, or communicative dimension or conception, for example. And one that targets standard musical features would presumably suffice for the task at hand, even though it would not for other investigations. As above, in my view, the music pluralist is better placed than the monist to give a coherent description of this situation in terms of 
music (unless, as I argued in chapter 3, the monist wields a bloated unitary concept, or makes ad hoc decisions about what counts as music simpliciter).

Consider another example of integration across levels of organisation. Probabilistic methods have been utilised in music perception and cognition research as well as in the modelling of musical structure, and Temperley (2014) argues that such formal methods provide promising avenues for studying the cognitive processes behind musical composition and musical expectation. Temperley's research question is something like the following: which probability model best predicts musical intervals? That is, hypothetically hitting pause on a melody, what is the probability that the next note is going to be a certain distance in pitch from the paused note? As for Grahn's research question, here too modelling will carry the explanatory force while other lines of evidence (data from actual musical form and experience) will play supporting roles.

In his article, Temperley distinguishes the Markov model of melodic interval, which defines a musical interval's probability 'by its count in a corpus, conditioned on previous intervals', from the Gaussian model of melodic interval, which defines it as 'a simple function of the size of the interval to the previous note and the distance to the mean pitch of the melody' (Temperley 2014, p. 96). The Markov model is characterised as statistical: it involves synthesising a large number of parameters from data collected; the Gaussian model is characterised as more rule-based, grounded in a few basic principles. These two models are then tested against several lines of evidence including sequential data from large samples of folksong, chorale, classical, and rock melodies, experimental data from the empirical study of melodic expectation, and distribution data capturing the melodic intervals comprising the Essen Folksong Collection (Schaffrath 1995). Temperley tentatively suggests that the Markov model is to be preferred for its lower cross-entropy, on all lines of evidence considered, than that of the Gaussian model (see Temperley 2014, pp. 90-92), however that's not the final word:

In terms of modelling the cognitive processes of composition: Does the better fit of the Markov model justify the view that composers maintain individual preferences for different intervals? Or, in view of the much greater simplicity of a Gaussian model, is it more plausible that composers 
simply favour pitches that are close to the previous pitch and to the centre of the range? (Temperley 2014, p. 96.)

Temperley invites theoretic debate about the weight that should be given to simplicity versus goodness-of-fit when evaluating these models of music. Plausibly, this reflection on theoretic virtues is to incorporate and integrate yet another level of organisation/degree of abstraction into the research agenda, though one that is playing a supporting role. For the same reasons as above, this situation lends itself well to a pluralist's conceptual framework.

Time to take stock. What have we gleaned from this brief foray into the scientific study of music? First, I have described music research along two rough dimensions, that of temporal scale and level of organisation/degree of abstraction. Second, I have described a plurality of lines of evidence which researchers draw on. Third, I have discussed the relationship between research questions and lines of evidence by considering the notions of explanatory force and supporting role. In my view, this situation is best conceived as a research cluster (Wylie 1999; see also Abrahamsen 1987). There is no single, homogeneous discipline of music's scientific investigation, but a messy cluster of researchers representing disparate research agendas, theories, and evidence-generating techniques and technologies. For such clusters to progress, attention must be paid to how the various investigations interface and how they could be integrated (systematically or otherwise). In my view, adopting conceptual pluralism about music can aid us in this integration. The pluralist is better equipped from the beginning than the monist to deal with the plurality of music concepts plausibly required by the legitimate research agendas that comprise this research cluster, focusing on differences in organisation/abstraction and temporal scale. To this end, in the following section I draw more explicitly on operational definitions of music, in the service of music pluralism.

\section{Operational definitions of music}

As I argued above, the scientific study of music requires contributions from a disparate, yet promising cluster of research questions and lines of evidence. In my view, there is no 
unitary, reductive, all-purpose definition of music appropriate for the needs of this pluralistic scientific enterprise. Indeed, as I shall discuss below, scientists rely on different operationalisations of music. These are non-equivalent, tailored to the research question at hand, and equally legitimate-why should the anthropologist's concept of music receive short shrift in favour of the neuroscientist's, or vice versa? However, this is not to assert that different music concepts are unrelated or wholly independent. I argue that music pluralism can encourage and drive scientific integration. ${ }^{38}$

Note that the tensions identified in chapter 3 reappear here. Music that does not meet Kania's intentional requirement might still realise the role of music in some sociocultural context and thus should be categorised as music by the ethnomusicologist, given their research agenda, but perhaps not by the aesthetician, given theirs. Similarly, music that does not meet a (Western) basic musical features requirement might still count as music for the ethnomusicologist and psychologist in certain contexts, but perhaps not for the (Western) music theorist. In short, there are multiple, non-equivalent concepts of music that are legitimised by their role in some research agenda. The appropriateness and applicability of an operationalisation should be evaluated with respect to the relevant research agenda at hand (as Kitcher 1984 argues with regards to species pluralism). Moreover, while some music concepts are complementary, others are antagonistic, concealing empirical disagreement. ${ }^{39}$

Recall my polysemy thesis about "sound" from chapter 2 . One way to illustrate complementary relationships between definitions of music is to recognise that scientists can study music—couched in the mode of sound—qua sound objects/events, sound waves, or sound sensations/representations. What a physicist or acoustician tells us about music, on their concept, can in principle be integrated with what a psychologist tells us about music, on their concept.

\footnotetext{
${ }^{38}$ It is possible to support different operationalisations yet think there is a unitary underlying concept. For example, there are lots of distinct measures for determining the phylogenetic diversity of a lineage or geographical location. The monist might claim that these are just different approximations or indexes of the underlying concept. I take my discussion in this section to show that at least some different music operationalisations target different roles best played by different properties, rather than approximate or index (some aspect of) a single concept.

${ }^{39}$ For a quick example, consider the interdisciplinary dissonance revealed in the musical versus linguistic syntax debate (Patel 2012a, b; cf. London 2012).
} 
An actual example of complementary relationships between distinct definitions of music can be found in Merriam's $(1963,1964)$ three-tiered anthropological conception of music, recently advocated by Cross (2012b). The first of these tiers is dubbed "concept"music's contingent, socially constructed guise, indexed to particular traditions and historical eras. The second tier is dubbed "behaviour"- the set of human behaviours from which the cultural manifestation of a musical tradition arises. Although two groups of people might share a musical behaviour - both might respond to rhythmic stimuli with spontaneous vocalisations or body movements-they might not share the same musical concepts. One group might perform music in a strictly ritually-loaded fashion. The other might limit it to mere entertainment. The third tier is dubbed "sound"- -the 'acoustical manifestation of musical practices' (Cross 2012b, p. 672). This tier targets aspects including timbre and musical scales-researchers might focus on how these vary cross-culturally, for example.

Three things to note about this example. First, these definitions are appropriate for researchers at different levels of organisation. Second, the three tiers, in my view, exemplify a complementary relationship between three music concepts-any could be the focus of a research question, and studies investigating phenomena through the lenses of these different concepts need not conflict. And third, the Merriam/Cross tripartite account could easily complement (or be complemented by) other concepts cooked up for other research endeavours: a concept specific to neural correlates or psychological modules springs to mind (e.g., McKeown-Green's “encapsulated module” suggestion mentioned in chapter 3). This pluralistic situation is not well accounted for by the monist's possible admission that the intersection of all these is music (simpliciter). Counterexamples, of which there undoubtedly are many (as pointed out in chapter 3), will suffice to call into question the usefulness of such a unitary analysis, and researchers are conspicuously lacking the motivation to make do with a problematic although univocal music concept.

Pluralism does not entail the acceptability of every definition of music. Some definitions underwrite empirical disagreement. In these cases, the definitions are in an antagonistic relation to one another. The researchers' understandings of music-or of what some aspect of music is-are at cross purposes and the disagreement calls out for resolution. 
I have already introduced Honing and Ploeger's distinction between musicality and music in the previous chapter:

We define musicality as a natural, spontaneously developing trait based on and constrained by our cognitive system, and music as a social and cultural construct based on that very musicality. (Honing and Ploeger 2012, p. 516.)

Contrast this distinction with Cross's way of distinguishing musicality from music: Cognitive science has dealt with music as though it consists of complexly patterned sounds engaged with through listening for their emotional or hedonic value, and with musicality as though it constitutes an autonomous domain of human thought and behaviour. Musicality seems to be interpreted as arising in two forms, one being the domain of the expert musician whose musicality is acquired partly through native talent and partly through extensive formal training, and who is responsible for producing music, while the other is broadly spread throughout the population, is manifested in the capacity to make sense of, and to be moved by, the complex auditory patterns that constitute music, and is acquired through processes of enculturation. (Cross 2012b, p. 669.)

Honing and Ploeger's musicality/music distinction splits the biological from the cultural in a hard-and-fast fashion; Cross's distinction does not.

In my view, these two music/musicality distinctions stand in an antagonistic relation with one another. They reflect conflicting theoretical and empirical commitments. Honing and Ploeger distinguish music from musicality in order to isolate aspects of the wider phenomena for explanation, but in doing so, implications follow about the causal dynamics in question. They assume that biology and culture can be harmlessly decoupled in theory. In the previous chapter I hinted that dividing musicality from music along biological/cultural lines might be unjustified, not least due to the dynamic nature of bio-cultural co-evolution. 
If the cultural and biological dimensions of musicality/music co-evolved in a tight-lock step, artificially splitting them would be misguided. I argue for this position in more depth in chapter 6.

On the other hand, Cross's definition of musicality is capable of encompassing both cultural and biological aspects of the phenomena, hooking onto tiers 1 and 2 of the Merriam/Cross tripartite account discussed above. Meanwhile, this definition of music targets the acoustic product of that tripartite account: tier 3. In my view, Cross's musicality/ music distinction should be preferred over Honing and Ploeger's.

In sum, then, the science of music should be recognised as a pluralistic conceptual pursuit. Investigative tasks calling for diverging music concepts divide relevant researchers/disciplines. The division is not an iron curtain, however. Complementary concepts breed studies that complement one another, and can be integrated with one another. They are appropriate concepts for different research agendas. On the other hand, antagonistic concepts clash and should be resolved. I have suggested that Cross's and Honing and Ploeger's conceptions of the music/musicality distinction stand in an antagonistic relation. Moreover, how scientists distinguish music from musicality in their operationalisations matters for scientific debate. Obviously, the claim that music is an adaptation, suitably distinguished from musicality, is significantly different to the claim that musicality is an adaptation. Thus in the following section I argue that not only can pluralism feed into empirical debate, it can make a difference: it can underwrite a consilience of evidence and provide a conceptual framework for progress.

\section{Pluralism in music-scientific debate}

In this section I lean further into particular debates within the scientific study of music. I argue that a pluralist's framework, generally, can aid progress.

I begin with a classic (following Killin 2017): is music innate? As we will see, the innate/acquired debate remains a pressing concern to many music researchers, part and parcel of traditional wisdom that distinguishes an organism's characteristics explained by its intrinsic nature from those explained by external influence (i.e., nature versus nurture). And it is commonplace to describe gifted musicians, colloquially, as "born that way", or to 
hear that their musical talent is "innate". According to Davis (1994), three-quarters of music teachers questioned in an informal British survey think that children learning music require innate talent in order to do well. Perhaps unsurprisingly, many scientists have rejected such a naïve nativist intuition (e.g., Howe et al. 1998). Some, for example, emphasise just how effective deliberate practice can be on musical expertise. Ericsson et al. (1993) report that top-level violin experts clocked up, on average, over 10,000 hours of individual music practice by the age of twenty, and that mid-and bottom-level violin experts clocked up 7,500 and 5,000 hours, respectively. All are serious investments of time and resources: even the lower end-5,000 hours - is over five years of three hours dedicated practice per day, six days per week.

Nonetheless, debate continues regarding the innateness (or otherwise) of various capacities that underlie/enable the production and perception of music, such as beat and tone perception-capacities widely distributed among the general population. The two main contemporary theories of the source of musical development privilege either innate psychoacoustic principles or models of exposure-based learning (see Loui 2012). Some theorists will tend to see aspects of musical cognition as focused and formed by domainspecific, innate faculties; conversely, others are sceptical that any aspect of musicality is truly innate. As we will see, this is an excellent case study for pluralism since not only should the investigation of music and innateness proceed in light of a plurality of music concepts, it should be recast in light of an orthogonal plurality of innateness concepts too. That is, I will suggest that whether music is innate (or not) turns not only on the music concept, or relevant aspect of that music concept, but also on the innateness concept at hand.

Winkler et al. (2009), for example, report their research into neonatal beat perception - the ability of infants to detect a regular, underlying pulse in music. They conclude that beat perception, given its early development, is likely to be innate. This is a premature conclusion, of course. As Grahn (2012) points out, a prenatal stage of auditory exposure/acquisition might explain the early development of beat perception. Consider that prenatal infants are exposed to a variety of external beats/pulses: the rhythms of the mother's heartbeat, for instance. And tone perception researchers suggest that neonatal preference for consonant musical intervals over dissonant ones is innate (see Marcus 2012; Trainor et al. 2002). Yet not only are prenatal brains capable of processing auditory 
information, they retain relevant information about musical stimuli. Empirical investigations have established that 'infants exposed to particular pieces of music before birth show distinct preferences for those same pieces after birth' (Sloboda 2005, p 67; see also Hepper 1991 and Parncutt 2009 for more on prenatal musical conditioning). So while neonatal beat and tone perception rely on the infant's inborn neural networks, they also rely heavily on auditory environment. And privileging just one of these, as innate/acquired theorising tempts researchers, is misguided.

As McDermott et al. (2016) argue, there is reason to think that musical culture plays a role in our tone perception, focusing on preferences for consonance. To test the claim that human preference for consonance over dissonance is innate, these researchers compared the response to note combinations of the Tsimane' people-a native horticulturalist-forager society living in the Amazonian rainforest in Bolivia, only very minimally influenced by Western culture - with that of town and city dwellers in Bolivia, and that of US residents. The Tsimane' people 'were mostly monolingual in their own language, lacked televisions, and had limited access to music via radio. The Tsimane' village lacked electricity and tap water, was inaccessible by road, and could be reached only by canoe... Their contact with Western culture was mainly limited to occasional trips to nearby towns' (McDermott et al. 2016, p. 1). Unlike the Americans and town/city dwelling Bolivians, the Tsimane' people did not show any preference for consonant intervals over dissonant ones, across several experimental studies. Variance in preference, rather, was explained by interval size, with higher preference ratings for larger intervals, not necessarily ones considered (by Western music theory) as consonant. These researchers conclude that their study 'suggests that consonance preferences are unlikely to be innate' (McDermott et al. 2016, p. 3); however, of course, they do not deny that biology plays a role in explaining the consonance preferences exhibited in, say, Western infants.

This brief sketch of these debates reveals a deeper issue about the innate/acquired distinction. As I hinted at above, perhaps the study of innateness itself ought to be conceived as a pluralistic enterprise, necessitating multiple non-equivalent reductions of "innate". And if so, if we are already music pluralists then we are well placed to take the possibility of a pluralistic treatment about innateness seriously too. 
First, innateness is a concept subject to much philosophical controversy. Mameli and Bateson (2006) round up twenty-six different definitions and distinguish eight distinct innateness concepts utilised by scientists. Presumably, then, there are several properties, and roles best captured by those properties, that researchers are attempting to elucidate by investigating so-called "innate" traits. The conflation of these properties has been dubbed the "clutter hypothesis" of innateness (Mameli 2008). Some philosophers (e.g., Griffiths 2002) have pointed out that proceeding by operationalising "innateness" for the research question at hand will enable progress, but will fail to elucidate innateness simpliciter, undermining the general innate/acquired distinction. Instead of using the term “innate", researchers might specify their research target explicitly for clarity: how developmentally robust the trait is, the extent to which it is altered through mechanisms of adaptive plasticity, whether it is environmentally canalised, whether it is highly heritable, or a statistical universal, and so on. For example, Ariew (2007) suggests that replacing ascriptions of "innate" with "environmental cannalisation" (the degree to which the endstate of a phenotype's development is buffered against environmental effects) will prove to be more useful to scientific modelling and debate than standard innate/acquired theorising.

Second, the brain's great plasticity means that in practice it is something of a challenge to distinguish innate from acquired neural circuits (McDermott and Hauser 2005), even on some specific operationalisations of "innateness".

Third, developmental interaction between genotypes and environments is both complex and ubiquitous. There is widespread agreement - the interactionist consensus (Sterelny and Griffiths 1999) — that a phenotypic trait's development hinges on both genes and environment. So although the intellectual climate has all but fully moved past a strict nature/nurture dichotomy (see e.g., Tabery 2014), the innate/acquired distinction remains, and although that distinction might be recast as a "matter of degree" by many researchers, its use, especially as unitary, threatens to draw attention away from the interaction of developmental resources. Although the brain is not a total "blank slate", there are no neural structures that develop without interaction with the environment. 
According to my pluralism, the project should not be whether music (or some aspect of music) is innate, but rather, whether music (or some aspect of music) qua some research agenda is innate qua some research agenda. For example, Hambrick and TuckerDrob (2015) argue that twin studies demonstrate that musical development is partially genetically influenced, partially environmental-and that genetic effects are accentuated in more musical environments. In particular, their findings indicate a nontrivial genetic effect on individual music practice (and that practice magnified those genetic effects) -a significant conclusion if true, since practice is otherwise conceived as a straightforwardly environmental variable (cf. Ericsson et al. 1993), pointing to an even more complex genetic and environmental interplay:

How might genes get "outside of the skin" to affect musical practice? It is likely that genetically influenced penchants and/or aptitudes for music could lead children to dedicate themselves to music practice, whereas children lacking such genetically influenced penchants and aptitudes for music might quit practicing early on, or never even begin. Similarly, children with penchants and aptitudes for music might evoke reinforcement from parents and teachers, leading them to be (even more) motivated to practice. Finally, genetic effects on practice could reflect personality or motivational factors... related to the propensity to engage in sustained practice. (Hambrick and Tucker-Drob 2015, p. 188.)

Hambrick and Tucker-Drob do not define "music" but ostensibly operate with a music concept suitable to psychological research, and one that explicitly targets deliberate, instrumental musical practice and music lessons. This obviously represents a narrower scope than that of other music concepts. But it does the job for Hambrick and TuckerDrob's investigative purposes. They were not "wrong” to focus on individual instrumental music practice and music lessons in their investigation of developmental gene-environment interplay, and have not misled their readers by doing so. Of course, taking a music concept more amenable to, say, anthropological or ethnomusicological research (as per earlier discussion) as the relevant benchmark would necessitate a refocusing of scope and a 
re-analysis and interpretation of the data, qua that music concept-different aspects of musical sociality and development would become salient than those of Hambrick and Tucker-Drob's investigation, in different cross-cultural contexts. And indeed it may be an open question how we should go about investigating traditional forager societies in which all members of the group participate in musical activities together, in this particular respect, rather than through solo practice and lesson-style teaching. But again, that's not to say that that these perspectives need be at loggerheads-two such music concepts are plausibly complementary insofar as they point to different (though related/overlapping) explananda, and once integrated, the conclusions from each would constitute progress. The burden falls on the monist to show how their unitary music concept allows them to agree in substance here and integrate research from a wide variety of research agendas.

So by operationalising the music concept under investigation clearly, the theories and evidence that support the "innateness" or otherwise of (some aspect of) some music concept can be made more transparent. Further work can then be done to plot research findings within the innateness clutter. The upshot: a messy, but promising route to plotting the ontogenetic career of music development. That some aspects of music picked out or made salient by some music concept are, say, genetically influenced or environmentally canalised to some extent, is plausibly compatible with some others being more or less so. I hope the above discussion of Hambrick and Tucker-Drob's case study shows as much. The tricky parts for future research are working out where the lines should be drawn, and asking which music concepts are most amenable to the research question at hand.

A philosophy of musics, as I have outlined it in chapter 3 , can weigh in on actual scientific debate here. One part of that programme involves relating music concepts to theoretic and empirical debate. I suggest that one way forward sees the development of hybrid models which elucidate the relationship between complementary music concepts, and integrating where possible the concepts and the evidence. I now turn to the area of comparative animal research to provide an example of how this might go, before turning to interdisciplinary research about musical rhythm.

Some researchers are interested in investigating evolutionary convergences. In the evolution of music literature, one key research direction targets vocal learning species: 
animals able to vocally mimic external sounds (various birds, cetaceans, pinnipeds, and humans-see e.g., Patel 2006). There is tantalising evidence that connects musical entrainment (bodily movement or gesture that corresponds to an external pulse or beat: from foot-tapping and head-bobbing to dancing) ${ }^{40}$ with vocal mimicry (Patel et al. 2009). Many vocal learning species are also entraining species. Human musical entrainment is of course a well-known, widely-dispersed ability. Infamous examples of non-human entrainment discussed in the literature include Snowball the sulphur-crested cockatoo and Alex the African grey parrot (see e.g., Fitch 2009) - two YouTube stars that spontaneously entrain to popular music tracks. ${ }^{41}$ Schachner (2013) reports that an extensive YouTube survey, exploiting a novel database, provides a sample of 33 entraining vocal mimics, and zero entraining non vocal mimics. And entrainment has not been witnessed in the wild (even among vocal learning species), suggesting that entrainment carries little or no adaptive fitness. ${ }^{42}$ Wild chimpanzees, bonobos, and gorillas "drum" away on their own bodies, and on tree buttresses and the like, yet they are not known to entrain. Entrainment seems to occur only in captive environments; indeed it may only be enabled by the cognitive offloading these environments allow (see Trestman 2015).

A hypothesis follows nicely from all the above: that entrainment is a by-product of vocal mimicry, or of the cognitive apparatus required for vocal mimicry (Patel et al. 2009). Nonetheless, it is evidently easy to misrepresent this hypothesis, as Stevens (2012) does: 'The broad hypothesis is that vocal learning species should be capable of synchronisation with a beat' (Stevens 2012, p. 658). This is not quite right; it is the reverse: that any organism capable of synchronising with an external pulse will also be capable of vocal mimicry, other things being equal. This prediction is testable and research is underway (see Schachner 2013).

If true, this hypothesis uncovers a dependency relationship between entrainment and vocal mimicry, through which researchers might gain insight into the development of

\footnotetext{
${ }^{40}$ More precisely, entrainment is the "coming together" and resultant synchronicity of multiple interacting, though independent, rhythmic oscillators or processes, not restricted to biological organisms. But I restrict discussion of it to this music-dance context ("musical entrainment"). ${ }^{41}$ See e.g., https://www.youtube.com/watch?v=cJOZp2ZftCw. Accessed 3/11/2015.

${ }^{42}$ This may be unsurprising, of course. There are few natural sources in the wild to which individuals or groups could entrain.
} 
these capacities. However, all may not be so well with the hypothesis. Cook et al. (2013) and Hattori et al. (2013) provide evidence of entrainment in non vocal mimics, though discussion is still open as to whether this undermines a dependency relationship in all cases (see e.g., Hoeschele et al. 2015).

An alternative hypothesis about entrainment has been proposed in the context of hominin evolution, the gradual audiomotor evolution hypothesis (Merchant and Honing 2014), which distinguishes interval-based timing (shared by non-human primates: Zarco et al. 2009) from beat-based timing (which builds upon interval-based timing, enabling musical entrainment). The idea is that although beat-based timing peaks in humans, it is present in a very restricted sense in other primates, and thus does not require vocal learning ability. There are a couple of cases of (again, tantalising) evidence that support this hypothesis. Although entrainment has not been witnessed in wild great apes, there are a couple of rare observations of it in captive chimpanzees. One chimp's drumming performance on an upturned bucket contained short patterns of rhythmic-beat regularity (Dufour et al. 2015) and another chimp's tapping performance on an electric keyboard reportedly synchronised with an external pulse (Hattori et al. 2013). So (some) chimpanzees have at least a latent sensitivity to external pulse, or so it seems. (I return to this discussion in chapter 7.)

This entrainment research tends to collect behavioural data. Few attempts have been made at collecting and integrating other lines of evidence, such as neural data (although see Honing et al. 2012; Merchant et al. 2015). Integrating this data can provide a platform for developing new research questions at additional levels of organisation (see e.g., Hoeschele et al. 2015), and weighing in on a potential dependency relationship between mimicry and entrainment (perhaps at least in some species-of course, if human entrainment turns out not to be a by-product of vocal mimicry this does not rule out the possibility that entrainment is a by-product of vocal mimicry in the convergent cases of certain birds and cetaceans, for instance).

Thus the study of vocal mimicry and entrainment in the non-human animal world could provide useful analogies for researching human music (for a discussion of the role of convergences in scientific research, see Currie 2013). If the analogies are tight, it may be useful to integrate or engineer a music concept that includes these non-human examples. 
After all, discussion of this outcome of convergent evolution appears often in theories of the origins and evolution of music (see e.g., Wallin et al. 2000; Morley 2013). This move is not immediately available to the monist who focuses on music simpliciter as humanlyorganised sound, or part of a specific institution or set of human practices, say.

A useful music concept might group together the vocalisations of vocal learning species but not the song of other vocal species, providing a useful concept for comparative research. This allows a non-arbitrary way to distinguish animals with "music" from those without for the investigative purposes at hand. The songs of gibbons and suboscine birds may be complex and aesthetically rewarding for humans to listen to, but these are not vocal learning species so for these purposes, their songs would not count as "music". This music concept in principle need not be in an antagonistic relation with music concepts utilised for other research that, say, limit the scope of "music" exclusively to humans. And it captures something important about human music too. Consider:

Perhaps the most obvious fact about human song is that it varies considerably between cultures, and much less so within cultures... That is, each culture has both a shared, open-ended repertoire of specific songs, and culturally specific styles or idioms that encompass multiple songs. This situation is only possible when songs can be learned-so a child or newcomer can absorb the song repertoire of its community-and new songs can be generated within the style. This aspect of human song therefore entails the capacity for complex vocal learning, where novel sounds can be internalised and reproduced... Having identified this particular 'design feature' of human singing, we can now ask which nonhuman species share this feature... As already noted above, many different species have independently evolved the capacity for complex vocal learning, providing a rich comparative database (Fitch 2015, p. 5.)

Returning now to the scientific study of human music, Fitch (2015) suggests that even aspects of music like rhythm and harmony might not be 'monolithic capacities' (Fitch 2015, p. 2). This suggests that pluralism has further applications in the science of music. 
Indeed, another example of pluralism, this time with regards to musical rhythm, emerges from the Journal of New Music Research's recent interdisciplinary issue 'espousing different but complementary views on musical rhythm' (Bello et al. 2015, p. 1). Here, cognitive science (Schaal et al. 2015), musical performance (Sethares and Toussaint 2015), ethnomusicological (Holzapfel 2015), music informational retrieval (Esparza et al. 2015), and signal-processing (Sephus et al. 2015) perspectives on the study of musical rhythm are presented. Schaal and colleagues, for example, define musical rhythm as 'temporal organisation of the musical material classified by the onset and duration of stimuli and the intervals between the onsets' (Schaal et al. 2015, p. 3), while Holzapfel distinguishes 'rhythm as a phenomenon inherent in music [from] meter as a mental construct that helps listeners to organise and memorise the temporal structure of music' (Holzapfel 2015, p. 25), while Sephus and colleagues focus on 'modulation spectral features' as 'temporal patterns, or rhythmic structure' (Sephus et al. 2015, p. 58). Note that Schaal and colleagues' account of rhythm is well-suited to their agenda. If rhythm is taken to be the combination of the duration of musical signals and the durations of the gaps between signals then it can be measured and is therefore a good target for the quantified experimental work of cognitive science. Ethnomusicologists, though, are interested in musical expression across culture, so dividing "rhythm" from "meter" along these lines allows them to target the relevant cultural aspect of their research. So although the authors representing distinct disciplines here do not target the same fine-grained concept, each identifies an aspect of musical rhythm for legitimate investigation, operationalising appropriately, and their research can in principle be integrated in the name of genuine progress.

The science of music is an interdisciplinary project. Again: divide, integrate, conquer. Difficult questions should be decomposed into questions about simpler targets-including, crucially, different music concepts, and in turn, aspects of phenomena targeted by those concepts. This plurality of research questions can be explored via different lines of evidence, and then synthesised into more holistic and multidisciplinary models that integrate complementary music concepts and evidential sources. Providing clear operationalisations of the music concepts, and evaluating their appropriateness for certain investigative tasks, is a prerogative of the research strategy that I have argued is vital for progress. 
To move debate forwards, music researchers must weave together many threadsinter alia evolutionary, developmental, behavioural, neural, psychological, social, and mathematical—into an integrated patchwork. These threads can involve higher or lower levels of organisation, research agendas at different levels of abstraction, and longer or shorter temporal scales. I have suggested that Wylie's concept of a research cluster fits this interdisciplinary research project, and that pluralism about music provides a promising conceptual framework for underwriting such a project.

\section{Coda: conceptual pluralism in aesthetics?}

In this chapter I have added to my argument for conceptual pluralism about music by demonstrating its utility in scientific practice (as a good philosophical naturalist ought).

Time now to sum up the last two chapters. A pluralist about some category argues that (1) there are multiple, non-equivalent concepts applicable to that category, and (2) that those non-equivalent concepts are legitimate. In diagnosing the failures of several unified analyses of music, and pointing to the music concepts relevant for different traditions, historic eras, and by different researchers, I motivated the first requirement. In my view, the equal legitimacy of the world's traditions/practices that give rise to different music concepts, and the research agendas that underwrite different operationalisations of music, motivate the second requirement.

In my view, there is no interest-independent fact of the matter about what music is. However, my pluralism is not a "free-for-all" relativism: one challenge is to understand which music concepts are admissible and which are not, which stand in complementary relations, and which stand in antagonistic relations.

Taking music pluralism seriously opens many doors for further inquiry, and the prospects are challenging yet invigorating. Which music concepts are employed, and by theorists representing which disciplines? Which are complementary, and which are antagonistic? And more generally: how can qualitative perspectives from the humanities be integrated with quantified, scientific research? Which lines of evidence bear on which research questions? 
However, defining music, and assessing definitions of music, is hardly the philosopher of music's raison d'être. (Indeed, in the next chapter, I turn to the debate about musical work ontology-perhaps the most discussed debate in analytic philosophy of music.) So before closing I should ask: does the discussion of conceptual pluralism about music have upshots for aesthetics more generally? I suspect so. I have shown how abandoning a search for a unitary concept of music can be both motivated and fruitful. Similar claims might be made of more central aesthetic concerns. Mag Uidhir and Magnus (2011) make the case for a pluralistic conception of art, and I am sympathetic. And although I have not made the case herein, beauty, sublime, and other key aesthetic terms seem to me to be potential candidates for pluralistic assessments. Just as I have been arguing for musics, not music, there may be beautys, and there may be sublimes. And if so, the scientific study of aesthetic perception/cognition, neuroaesthetics, evolutionary aesthetics, and so on, might in fact belie multiple, non-extensionally equivalent, though equally legitimate concepts. Obviously, the plausibility of this suggestion requires an analysis of scientific investigations into aesthetics.

My method for a philosophy of musics, if applied more generally to aesthetic concepts, might even pave the way forward for a "less elusive" analytic aesthetics research programme than is commonly suspected about aesthetics by other philosophers. Consider Goehr's pessimistic take on the current situation:

With the predominance of science, aesthetics has tended to stand on the borders of disrepute. Aesthetic concepts, perhaps of all concepts, are believed to be least subject to scientifically styled or logical description. (Goehr 2007, p. 71.)

In my view, taking conceptual pluralism seriously in aesthetics generally might assist analytic clarity as researchers can agree (or not) on a plurality of conceptions and distinctions for a plurality of purposes. Whether or not these suggestions bear fruit depends on future research. 


\section{Chapter 5: Musical works}

In the last two chapters I argued for conceptual pluralism about music. In this chapter I bring my pluralistic perspective to bear on the divisive and extensively-debated topic of musical work ontology. Operating here with a relational conception of music, I hone in on one particular musical work concept: the Western classical musical artwork; that is, the work concept associated with the Western classical art music tradition/practice/discourse. I advocate and develop a version of fictionalism about the ontological category specified by that concept. The idea is not that certain concrete particulars like a musical performance (or a composer's manuscript, or the mental representation of a sound-event gleaned through reading a score-copy and imagining a performance, and so on) are thereby not art objects of some description, worthy of aesthetic evaluation and attention. As far as I am concerned, these things exist and are salient candidates for artistic/aesthetic evaluation. But I suggest none of these things realise the elusive musical work concept. Sometimes we might speak of these things "as if" they are musical works. But when we seem to be speaking of some additional entity, "the musical work so-and-so", above and beyond performances, scores, imaginings, and so on, it is my contention that, strictly speaking, we fail to refer. Nonetheless I shall argue that, once re-conceptualised as a useful fiction, we need not revise our concept of Western classical musical artworks (just the broader context, philosophically speaking).

\section{Musical works and ontological theorising}

The metaphysical status of musical works is one of the most debated concerns in analytic philosophy of the arts. Participants in this debate focus on musical works from the Western art music tradition. This is often explicitly noted. For example, Levinson states:

I am confining my inquiry to... the fully notated 'classical' composition of Western culture, for example, Beethoven's Quintet for piano and winds in E-flat, Opus 16. So when I speak of 'musical work' in this paper it should be understood that I am speaking only of these... (Levinson 1980, p. 6.) 
I will follow suit. By asking "what are musical works?", and making the necessary disambiguation, my question here is: what entities, if any, realise the target role specified by the relevant work-concept? Philosophers want to know the ontological category of the thing in common-if it is anything at all-that composers create, performers instantiate, music theorists analyse, critics evaluate (e.g., "The Jupiter is beautiful, but last night's was a dreadful performance of it"), and composition professors assess and grade. Historically this has been a salient concept in philosophy of music and discussion of it is both lively and prolific (as I will outline later). The ontology of other musical systems/practices/traditions is usually discussed separately, for instance see Kania (2006) and Gracyk (1996) on the ontology of rock music.

This is an example of the pluralistic approach that I think is necessary for progress: not to assume straight off the bat that a unitary musical work concept extends to all music domains - to all things loosely conceivable as musical entities—or that all such objects are ontologically homogenous. I suspect it may be nothing more than a convenience of language that we call them all "musical works"-perhaps musical work is a very general and equivocal concept, containing a plurality of ontological categorisations.

So, henceforth, by "musical works" I explicitly intend to pick out only the works of the Western classical art music canon. And there are a bewildering number of ontological accounts of these on offer. Philosophers have argued that musical works are such things as compliance classes, abstract objects of various stripes, acts of composition, perduring existents, enduring existents, historical individuals, and mental entities. There is little consensus regarding this matter and debate continues in the academic literature. These views are surveyed in $\S 4$.

In my view, the debate reveals a tension between philosophical theorising and musical practice-how philosophers think we ought to conceive of musical works in order to acquiesce in a neat general theory, on the one hand, and how musical works are actually conceived within the musical practice, on the other. This yields two broad philosophical methodologies for theorising about the work-concept at hand, loosely captured by the descriptive/normative metaphysics distinction (Strawson 1959). 
Descriptive metaphysics is concerned with producing a plausible theory of (some aspect of) reality that coheres with the actual concepts under scrutiny (with perhaps a few minor concessions reached through reflective equilibrium). Normative metaphysics is concerned with producing the metaphysically "best" theory of (some aspect of) reality, largely irrespective of our concepts, requiring often massive conceptual revision of the target(s) of inquiry. Of course, the notion of "best" is elusive here; nonetheless, the two broad strategies reflect different underlying explanatory aims and commitments. Normative metaphysical theories are lauded by their authors for their theoretical virtues (e.g., neatness, simplicity, unification, generalisability), while coherent descriptive metaphysical theories are lauded by their authors for their success at cashing out a concept that is actually utilised by-and with any luck, actually matters to-the participants of the target practice or discourse.

This distinction is useful (descriptive metaphysics is about one's actual beliefs; normative metaphysics is about the beliefs one should have), but these approaches are not mutually exclusive. Indeed, in practice, metaphysical theories offered by philosophers have descriptive and normative aspects, and Kania (2008) points out that few (if any) musical work theorists are of an exclusively descriptive or normative ilk. In my view, we may conceive of this distinction along a continuum: if theoretic considerations are allowed a prominent role in the analysis, we shift to the normative side; if conformity to the users' practice is weighted heavily, we shift to the descriptive side. I will suggest that the (realist) ontological accounts that lay on the normative side do not address the work-concept that I have set out to explore: they ascribe properties to musical works that are not only counterintuitive but contrary to how the concept is utilised in practice. That is not to say that these views do not describe something at all, but that that something (if it exists) is not what plays the role specified by the musical work concept under scrutiny. I will also suggest that the (realist) ontological views on the side of descriptive metaphysics are sui generis, ad hoc, or metaphysically suspect - the categories specified have no place in a naturalistic ontological framework. So positing them is not independently motivated. Thus there is a stalemate, of sorts. Given this stalemate, I motivate and defend my fictionalist alternative: the descriptive element of my view retains the musical work concept as utilised in the 
target practice/discourse; the normative element does away with ontological commitment, but not in a way that is damaging to the practice/discourse.

Thus my approach leans towards the side of descriptive metaphysics. I endorse David Davies's pragmatic constraint (D. Davies 2004). That is, the view that I end up advocating should cohere at least conceptually, more or less, with the target musical tradition/practice/discourse. Of course, what counts as cohering is difficult to specify. But at the very least, it should not be hostage to a philosopher's general metaphysical model. The account I develop will require some "philosophical" revision, admittedly (this is the normative aspect), but this revision will do no damage to the conception of musical works at home in Western art music practice and discourse.

Many philosophers writing about musical works (or artworks in general) are beginning to lean on this side of the methodological divide and I think this is a step in the right direction. (Indeed, there is growing unrest about normative musical ontology: see Kania 2008; D. Davies 2004; Rohrbaugh 2003; Thomasson 2006.)

Consider some of the recent defenders of the descriptive agenda: ... the ontological status of works of art is, at bottom, fixed by human intentions and practices. The ontological status of such general, familiar art kinds as paintings and symphonies is established by the beliefs and practices of those who ground and reground the reference of these general terms. The ontological status of an individual work of art is determined, at bottom, by the artist's (actual or intended) sanction. (Thomasson 2010, p. 128.)

[Musical works] are what they are in virtue of our creating them and the practices that have evolved concerning them. The nature of these objects can only be found in our intentions and practices regarding them. (Stecker 2009, p. 382). 
Artworks must be entities that can bear the sorts of properties rightly ascribed to what are termed "works" in our reflective critical and appreciative practice; that are individuated in the way such "works" are or would be individuated, and that have the modal properties that are reasonably ascribed to "works" in that practice. (D. Davies 2004, p. 18.)

Ontologies of art are beholden to our artistic practices-the ways we talk, think, and act in relation to art or at least some rational reconstruction of these-and the critical debates are part of the practices to be captured. (Rohrbaugh 2003, p. 179.)

... a successful theory... is one sensitive to the roles the work-concept has (and does not have) in practice. It is one that explains what sort of thing a work is in terms compatible with the description of how the concept of a work functions in practice. It would demonstrate that thinking in particular theoretical terms enables one to illuminate the phenomena one wants to account for... (Goehr 2007, p. 20.)

This chapter plays out as follows. In $\S 3$ I advocate the Canberra Plan methodology for a descriptive metaphysics of musical works. I explicate this method, provide some examples, and apply it to the musical work concept. This leads me to fictionalism about musical works. Unlike the various realist theories that I survey in $\S 4$, fictionalism is neither metaphysically suspicious, nor does it require massive conceptual revision (the revision required is of the wider conversational context, philosophically speaking; a small bullet to bite). So in $§ 5$ I outline fictionalism in general and I cash out my brand of fictionalism about musical works. But first I must reflect a little more on the work concept at hand. 


\section{The musical work concept}

I have already stated that my discussion is restricted to talk of the works from the Western classical art music tradition; that is, the musical artworks that are performed in concert halls and chambers by symphony orchestras, string quartets, and so on. Lydia Goehr (2007) provides an historical narrative, supplemented by a wealth of historical data, of the emergence and influence of this work-concept throughout the classical period (c. 17501800), and its coming to fruition—as a regulative concept-around 1800.

The concept is regulative in the sense that it underwrites a social norm amongst classical musicians: in giving a serious performance, classical performers must "submit" to the musical work; they must strive to be true to the work, strive to perfection in performing the work. The work is conceived as fixed; encoded in its score-copies (which, admittedly, can be more or less specific about various features) and exemplified in its performances. Despite the different aesthetically-relevant interpretations that performers and conductors bring to their musical performances, the musical work itself is conceived as unchanging and the same across these different performances.

This situation already distinguishes the relevant work-concept from others. Performers of jazz standards might have no such inclination to "submit", diverging widely in their renditions of standards and standard-based improvisations. And folk songs from oral music traditions diffuse and evolve over time.

Indeed, Goehr allows for the detachment of the work-concept under consideration from that found in improvised jazz (see p. 31), and in her introductory essay, she seems to endorse a pluralistically-compatible perspective, 'the work-concept should not be assumed naturalistically or essentially to exist in all music practices of whatever sort' (Goehr 2007, p. xlviii).

But what, according to this concept, are musical works identified as? Goehr's answer, which I shall take as my benchmark, is:

[Musical works are conceived as] objectified expressions of composers that prior to compositional activity did not exist... original, unique products of a special, creative activity... symbolically represented by composers in scores. 
Once created, we treat works as existing after their creators have died, and whether or not they are performed or listened to at any given time. We treat them as artefacts existing in the public realm, accessible in principle to anyone who cares to listen to them. (Goehr 2007, p. 2.)

Yet this analysis does not straightforwardly specify some noncontroversial ontological category. To draw the point out a little further, consider the following thought experiment, loosely adopted from Kivy (2002). You have turned on the television to watch the morning news. The headlines begin, only to announce that the sculpture The Thinker has been abducted from the gardens of the Musée Rodin. Although the best French sleuths are on the case, you are told that they have no promising leads. An accompanying tag-line scrolling along the bottom of the screen reads "Thinker pinched, cops baffled". Although this theft presents an intriguing puzzle to be solved, there is nothing metaphysically peculiar about it. This is because sculptures are physical things. Insofar as they are artworks, one might say they are autographic, corresponding in some salient way with their physical matter (though philosophers will presumably disagree about how this is to be cashed out).

Compare this with the following example. You have turned on the television, only to read this tag-line: "Beethoven's Fifth Symphony pinched, cops baffled". For starters, it is baffling to grasp exactly what is thought to have been stolen in this case. Unlike the statue example, it is not so obvious what the musical work, "Beethoven's Fifth Symphony", actually is. ${ }^{43}$ Indeed, Kivy dubs musical works 'ghostly apparitions' (Kivy 2002, p. 206); they are thought to be the kind of entity that philosophers have called 'exotic' (Sainsbury 2010, p. 23), 'obscure' (Goehr 2007, p. 2), or even ‘ontological mutants' (Tormey 1974, p. 207). They are not considered autographic, but allographic (see Goodman 1976).

Of course, there are score-copies and recordings of Beethoven's Fifth Symphony that could be stolen. These correspond with physical matter. Even a performance of a

\footnotetext{
${ }^{43}$ It is perhaps worth pointing out that the same criticism could be levelled against other purportedly abstract objects: numbers, propositions, and so on.
} 
musical work, or a playing of a recording of a musical work, is a physical thing. ${ }^{44}$ But none of these things-score-copies, performances, recordings, playings—is straightforwardly captured by the musical work concept. (For example, intuitively, destroying all the scorecopies would not destroy a musical work.) This has been pointed out by philosophers many times before (see e.g., Kivy 2002), and this at least is uncontroversial.

So, Rudner, for example, maintains:

[A musical work] is no particular rendition of a score. It is not the actual physical sound waves which constitute any rendition; it is not the printed score from which the orchestra is playing; and, curiously enough, it is not what we might normally call the phenomenal content of the mind - that is to say, it is never any particular heard music. (Rudner 1950, p. 380.)

Consider the following sentences: "Hammerklavier is a piano sonata". "Beethoven's Fifth Symphony is older than Beethoven's Ninth Symphony". "Beethoven composed the Eflat Quintet for piano and winds, Opus 16". "Für Elise is many people's favourite piano work". Taken ordinarily, I contend that these sentences are not about particular scores, performances, mental representations, or the like. Rather, speakers of these sentences intend to refer to something in addition to these things. And so it is this "something else" that is said to be the musical work, and thus the intentional object of these sentences.

Distinguishing works from displays will help here: 'a work's display is a structure that results from the artist's creativity and that we apprehend in order to grasp a work's meaning and aesthetic qualities' (Lopes 2010, p. 4). Score-copies, performances, playings, recordings - these are all kinds of displays. The performance of Hammerklavier one night is a distinct display from its performance another night. Displays are straight-forward concreta, easily accounted for in a naturalistic ontological theory. Works, however, have given rise to a number of competing accounts, as I shall soon explain. But first, in the next

\footnotetext{
${ }^{44}$ By "performance" I mean a live, real-time occurrence of music produced by performers; by "playing" I mean the playback of a recording; by "recording" I mean the medium for stored information—on vinyl, CD, mp3, and the like-of the captured performance, or simulation of a performance.
} 
section, I suggest that a methodology hitherto unapplied within this debate (as far as I am aware) will assist progress: the Canberra Plan.

\section{The Canberra Plan}

The Canberra Plan (see Jackson and Pettit 1995; Jackson 1998; Braddon-Mitchell and Nola 2009) offers a naturalistically-respectable framework for unpacking the musical work concept. The Canberra Plan is naturalistically-respectable since it follows a general scientific method: it defines the concept under scrutiny and generates a hypothesis which researchers attempt to validate by locating the target postulated in a plausible theory. If they can do so, metaphysical success! If not, failure (error theory).

The first step of the Canberra Plan is to collect the platitudes associated with the concept under scrutiny. A platitude is a proposition about a concept that is understood by proficient representative users of that concept as uncontroversial. Concepts acquire their application conditions via the constellation of concepts and terms with which they interrelate in a given context or discourse. And in acquiring our concepts, we acquire sets of dispositions which interface between statements expressed in terms of some concept with statements of other things. These dispositions underwrite the platitudes about that concept. Take belief as an example. Following Papinau (2009), the belief platitudes might be "caused by perceptions", "combines with desires to generate actions", "has causally significant internal structure".

The second step is to systematise those platitudes as an implicit definition of the concept in question. Again, take belief as an example. This might look something like:

A belief is 'some unique kind that is characteristically caused by perceptions, combines with desires to generate actions, and has causally significant internal structure.' (Papinau 2009, §2.3.)

The third and final step: see if anything in the world plays the role specified by the concept. For belief, that means identifying the 'internal states that play the causal role associated with the concept of belief' (Papinau 2009, §2.3). 
So far, so good. But sometimes the search might turn out empty-handed. In this case one option is to seek a best-filler (Jackson 1998). That is, perhaps nothing plays the role specified by the concept, but some other thing comes so close that it can, for all intents and purposes, be conceived as the target of the concept. For example, if van Inwagen's (1990) compositional nihilism is right, then there are no tables and chairs, but rather simples arranged table-wise and simples arranged chair-wise. If that is the case, perhaps simplesarranged-table-wise is the best-filler for the concept table. It would probably make no difference, outside of the philosophy room at least, if we all were picking out simplesarranged-table-wise when utilising our table concept.

Alternatively, perhaps the concept under scrutiny is just an empty concept. A horse with a horn tied onto its head does not fill the role of unicorn: unicorns just do not existnothing comes close to playing the role specified by an implicit definition underwritten by the unicorn platitudes, something like "horned horse-like quadruped, aggressive though elegant, white in colour, docile only to virgins". And substituting anything else in would be irrelevant or otherwise misguided. Of course, this is not to deny that unicorns appear within fictional stories-readers and listeners of these fictions have a shared concept of unicorns. But there are no such things as unicorns out in the world. Ideas or representations of unicorns are not unicorns—ideas are not aggressive, horned quadrupeds docile only to virgins.

What about the musical work concept? Any competent wielder of the musical work concept representing the Western art music tradition/practice/discourse accepts the following platitudes:

Musical works are created by their composers.

Musical works are created at or around the time the composer completes his/her creative processes. (Considerations might include the composer having formed a judgment or disposition about it being complete, but the specifics are irrelevant here).

Musical works are exemplified in their (correct enough) performances and playings. Musical works are encoded in their (correct enough) score-copies and recordings. 
Musical works can be accessed by people through hearing performances/playings, or through reading a score-copy and imagining a performance. (In other words, you can listen to a musical work; you can glean it from a score-copy.)

Musical works are not the same as performances, score-copies, recordings, or playings, or mental representations of those things.

Musical works can be ascribed distinct properties than those ascribed to particular performances, score-copies, recordings, and playings.

A musical work could have been different, if the composer's creative activity resulted in something slightly different. (A musical work could have ended with a major instead of minor triad (a tierce de Picardie), say, had the composer desired it—in other words, musical works are modally flexible.)

These platitudes reveal an implicit description of how the musical work concept is conceived and I take them to be consistent with Goehr's historical narrative and entrenched in the target tradition/practice/discourse. ${ }^{45}$ That is, that a musical work is an entity that is created by its composer, completed around the time of the composer's creative activity, encoded in its score-copies, exemplified in its performances, and so on. These platitudes thus describe the entities that an ontological theory must locate, in my view, if it is to locate musical works. So in the next section I survey the leading accounts on offer. I suggest that the search fails to turn out an adequately persuasive result. Then in the subsequent section I suggest that the musical work case is somewhat like the unicorn case-that of an empty concept-yet that "musical works", as conceptualised by the target tradition/practice/ discourse, populate a useful fiction, so to say, so we need not drop our thought and talk about them.

\footnotetext{
${ }^{45}$ Note that these platitudes are not automatically generalisable to other musical work concepts, and vice versa. For example, it is a platitude of at least some Inuit peoples that songs carry the "inalienable soul" of the composer (Sachs 1962). It is a platitude of Yirkalla Aborigines of Australia that songs are discovered (for instance, in the babbling of babies), not composed (Ball 2010).
} 


\section{Ontological accounts of musical works}

In this section I survey the leading ontological accounts of musical works. There are a bewildering number of accounts on offer, and they have been extensively discussed and critiqued in the philosophy of music literature before (see e.g., Kania 2012b; Dodd 2007, 2008; Kivy 2002; Livingston 2011; Rohrbaugh 2005). So it is not my intention to be exhaustive or excessively detailed in covering well-trodden, familiar ground. I provide a schematic, table-like discussion that lists the main accounts, key references, and a few key objections to each. 'The tempo is allegro' (Davies 2003, p. 30).

These accounts are all realist theories. ${ }^{46}$ In other words, they all agree on one thing at least: that musical works exist; that musical works are a part of reality, whatever they are. (Later, I depart from this presupposition; for the time being, my narrative tone will reflect the fact that the participants in the debate conceive of musical works as existent.)

I distinguish three broad groupings of what I am calling "realist theories" of musical works, and I present the various accounts of musical works according to this taxonomy. The grouping is heuristic and not meant to be any more than a convenience. First, nominalist/reductionist accounts: these identify musical works with concreta—or with collections of concreta (setting aside the orthogonal project of assessing strategies for nominalising/reducing thought and talk of things such as sets). Second, abstract object accounts: these identify musical works with instantiable abstracta. Third, novel accounts: these theories identify musical works with actions, historical individuals, abstract artefacts (non-instantiable abstracta), and mental entities.

\subsection{Nominalist/reductionist accounts of musical works}

Musical works are sets (or compliance classes) that contain the accurate musical performances of score-copies (Goodman 1976).

On this view, Mozart's Jupiter, as a compliance class, would be completed with its last ever performance, by those performers, and not with the completion of Mozart's

\footnotetext{
${ }^{46}$ Anti-realism about musical works has hardly been discussed in the philosophical literature, truly dwarfed by the prominence of realist theories. Dodd's (2008) recent literature review does not even mention anti-realism as a possible position.
} 
creative act (or his forming of a completion disposition or however else we might specify Mozart's completion requirement-see e.g., Trogdon and Livingston 2014). Yet according to practice, Mozart's Jupiter was completed by Mozart, in 1788.

Scored but unperformed/unrealised compositions would all be identical — the same musical work—because their compliance classes are all empty.

Electroacoustic musical works are an uncontroversial part of the Western art music tradition of which many are not scored.

The wrong note objection: at least as Goodman renders the account, it excludes imperfect (non-compliant) performances, no matter how minor the imperfection may be. But almost every performance will contain at least the slightest deviation from the score, and in practice these are conceptualised as performances of the relevant musical work (Trivedi 2008).

More generally, the account is motivated by considerations of Goodman's extensionalist theory of symbols in a "languages of art" framework (for critique see Goehr 2007). It is divorced from considerations of musical artistic practice. About the disconnection, Goodman pines 'one hardly expects chemical purity outside the laboratory' (1976, p. 186). Yet outside the "laboratory" there is hardly a "chemical" of the kind at all: the target of inquiry must be entirely reconceptualised.

Musical works are perduring concrete entities: the mereological fusions of their performances (i.e., each performance is a temporal part of the musical work, which is the sum of these temporal parts) (Caplan and Matheson 2006).

Caplan and Matheson draw an analogy with perdurantism about people, which they claim is a standard and reasonably well-accepted view about personal identity. But the comparison to musical works might not be as strong as they claim. Barring awkward scenarios such as discontinuous time-travel cases and so on, people exist at all times between the moments of their birth and death (roughly). Presumably this is so because at every moment, a person stage exists. But a "musical work stage" does not persist in the 
same manner-there is not a continuous performance of Jupiter from the moment of its composition. There may well be no performance anywhere of Jupiter at this very moment, for example. So Caplan and Matheson need to explain what connects/fuses the distinct performances together since they occur at different times, without continuous and linear persistence.

Musical work stages are not causally linked in the same way that person stages are linked. Me-now is a causal descendant of me-just-before-now. Last night's performance of Jupiter (in, say, Auckland) is not a causal descendant of last week's performance of Jupiter (in, say, London, by a different orchestra, under a different conductor, and so on).

Moreover, unlike people, performances could be happening at the same time in two different spatial locations, London and Auckland, perhaps. Barring awkward time-travel scenarios, people cannot exist simultaneously in London and Auckland.

As with Goodman's account, the musical work would not be completed by the composer at the time of the composition's completion. And unperformed/unrealised scores have empty fusions and thus would not encode musical works.

A perduring entity grows bigger, fusing more temporal parts as more performances are given. But musical works do not enlarge with every performance.

Musical works are enduring concrete entities, wholly (rather than partially) located at any region occupied by a performance (Tillman 2011).

This account straightforwardly identifies musical works with performances. Yet people can be intimately familiar with a musical work —and even admire it (Martin 1993) — through studying score-copies, for instance.

The properties a critic might wish to attribute to a musical work and various performances thereof may be distinct (and this seems to undermine transitivity of identity and potentially lead to contradictions, if each performance is identical to the musical work). Unperformed/unrealised scores would not encode musical works. 
Summary

1. These nominalist/reductionist theories do not capture the target specified by the musical work concept. Insofar as they describe anything at all, it is not what we are probing the world for, given our platitudes/implicit definition.

2. While it may be useful to talk of (the physical counterparts of) musical works in the language of compliance classes, perduring entities, or enduring entities for the purpose of philosophical theorising (which also it may not be; this remains to be demonstrated), this requires revision of the musical work concept. The ontological category specified by the musical work concept under scrutiny remains unaccounted for by these theories.

\subsection{Abstract object accounts of musical works}

Musical works are Platonic (eternal) abstract objects (i.e., Forms/types). Performances and playings are concrete tokens of that abstract Form/type (e.g., Dodd 2000, 2002; Kivy 1983, 1987, 2002).

On this account, musical works are implausibly timeless, predating even any sentient life-and thus are not created by their composers. And since they populate the inaccessible nether-regions of the Forms, it is puzzling how we should have any epistemic or experiential access to them.

Platonic types are modally inflexible, whereas musical works are conceptualised in practice as modally flexible-they could have been different to what they in fact are (see Rohrbaugh 2003, especially p. 185).

The wrong note objection arises here too: a performance is not a token of the relevant Form if it is imperfectly rendered. Indeed, this account may result in an extremely profligate ontology of musical works qua sound-event structures (see Young 2011, especially p. 296)—every possible musical work must already actually exist.

If two composers independently hit upon the same pure sonic structure then according to Platonists, they have hit upon the same musical work. Yet their works could 
still exemplify distinct aesthetic properties (or so says artistic practice). Since they might be composing at different times and in different socio-cultural conditions, it is possible for critics to ascribe one composer's (say, rather dry) work with seriousness and lifelessness, and the other composer's (pastiche) work with properties like irony and sarcasm, and that these properties are understood as features of the work.

Musical works are Platonic (eternal) norm-kinds/norm-types (Wolterstorff 1980; see also Dodd 2007).

Some philosophers add a normative aspect to their Platonic account of musical works in order to dodge criticisms like the wrong note objection. (Accordingly, although a performance of some musical work ought to go thus-and-so, it might still count as a performance of that work even if it does not.) But this addition seems misguided. The physical acts of performances of musical works may well have a hypothetical-normative aspect (regulated by a social convention: that for this kind of music, performers ought to best follow the score) but why think the normativity is categorical and moreover transitive, metaphysically, to the musical work itself? Performances might be "malformed" not because the musical work is a norm-kind, but because of normative aspects of the classical music practice/tradition.

Musical works are Aristotelian universals: abstract essences/norm-kinds instantiated in their performances (Walton 1988; Davies 2003).

Aristotelian universals exist "in" their instances. So the musical work would come into existence with the first performance (first "tokening" or "instantiation"); not with the completion of the composer's creative act.

Moreover, on this view, an instance of music (an improvisation, perhaps) might token a work that will be composed in the future, ante-dating the identity of the musical work (Rohrbaugh 2003).

Unperformed/unrealised scores would not encode musical works. 
Musical works are initiated/indicated abstract objects: a "structure-S-as-composed-by-C-at-t" (Levinson 1980; Trivedi 2002).

This account is little more than not-very-subtle sleight of hand. It postulates a category of sui generis abstract objects without independent philosophical respectability, and has been criticised for doing so:

How is this [a structure-S-as-composed-by-C-at-t] supposed to produce a new entity, as opposed to merely establishing a relation of interest between a thinker and the object of her thought?... One might doubt that Levinson has successfully explained how new types come to be through acts of indication. (Rohrbaugh 2003, p. 194)

... it does not appear to be true in general that, whenever an agent $a$ enters into a relationship $R$ with an object $o$ at a time $t$, a novel entity comes into existence, one which may be denoted by an expression of the form 'o-asR'd-by-a-at-t'. For instance, in the absence of evidence to the contrary, it does not appear to be the case that, if you show me the tallest building on campus you thereby bring into existence a new object, that is, the buildingas-shown-by-you. (Predelli 2001, p. 289)

Since even initiated/indicated types exist non-spatio-temporally, and are thus beyond human causal interaction, it has been argued that they cannot through human action/agency be brought into existence (Kivy 2002).

And types such as these may not even be logically coherent postulations. For example, Dodd argues that 'types inherit their existence conditions from their propertyassociates, so, since properties are eternal, so are types' (Dodd 2002, pp. 389-390). In other words, an abstract object $x$ exists if and only if the property-associate being-an-x exists, and if such a property-associate exists, then according to Dodd, it exists eternally, and thus $x$ exists eternally. 


\section{Summary}

1. These abstract object accounts of musical works do not capture the target specified by the musical work concept. As with the nominalist/reductive accounts, insofar as these accounts describe anything at all, it is not what we are looking for.

2. About the categories identified by these theories, frustratingly we can only speculate. Perhaps these theories capture something: perhaps the (Platonic) abstract structures of musical works, if such things exist, or their (Aristotelian) essences. After all, the universalia in rebus versus universalia ante rem debate has persisted since philosophical antiquity. And I have little new to say on that debate; it is not my concern, if these categories do not cohere with the musical work concept.

3. Moreover, unlike the nominalist/reductive accounts which specify concrete counterparts to musical works, it is not obvious how much work the abstract object accounts of musical works can do in a naturalistic research framework: given the principle of causal closure, one of the central tenets of naturalistic science (for all physical events there is a sufficient causal physical explanation), abstract objects are explanatorily irrelevant so not worth positively arguing for.

\subsection{Novel accounts of musical works}

Musical works are action-types: the composer's creative act type (Currie 1989).

This is strikingly counterintuitive. It identifies the musical work as the abstract type of the composer's action sequence. Any given action sequence token comprises the effort/labour of making the thing we usually conceptualise as the musical work (creative output of that effort/ labour), so this account changes the subject completely. And in any case, abstract types are not created by their "composers"; are modally inflexible, yet musical works are not, and so on. For more criticism and commentary, see D. Davies (2004). 
Musical works are action-tokens: the "performance" of the composer's creative act (D. Davies 2004).

Similarly, this is strikingly counterintuitive. It changes the subject from the creative output of the composer's labour to the actual labour token itself. For example, on this account, people cannot experience a musical work by attending a concert (Kania 2005), contrary to our platitude. Similarly, familiarity with a musical work would be familiarity with the ins and outs of the relevant actions of the composer: you couldn't glean it from reading a score-copy.

Musical works are historical individuals (Rohrbaugh 2003, 2005).

Kania calls this account 'highly intuitive, but metaphysically unorthodox' (Kania 2008, p. 433); Dodd (2007) argues it is not well motivated and that it does not allow for the intuition that musical works are repeatable. (For more objections, see Dodd 2007, chapter $6)$.

Musical works are abstract artefacts (Thomasson 2006).

Since artefacts are particulars, it is puzzling how an abstract particular could be embodied in its "occurences" (concrete particulars). Unlike on the standard abstract object accounts, on this account musical works are not instantiable. The objection is that this feature of Thomasson's account is a bug and not a bullet worth biting, especially if abstracta are already superfluous to the naturalist's explanatory aims (again, consider the causal closure principle).

Like Levinson (1980; see above), Thomasson seems to have invented an ontological category to match her description of musical works. Bertrand Russell cautions against 'The method of "postulating" what we want' (Russell 1919, p. 71) without adequate independent metaphysical defence. Kania (2008) suggests that perhaps Thomasson simply fails to refer when she speaks of musical works, rather than succeeds in referring to a sui generis abstract entity. 
Musical works are mental entities (ideas or representations): mental objects or mental experiences (Cox 1986; Collingwood 1938; Sartre 1940).

On these accounts, musical works would lack intersubjective accessibility. If musical works are ideas, there will be as many Jupiters as there are people that have the idea. ${ }^{47}$ And people can have very different ideas or phenomenal experiences of the same "thing": idealism does not seem to account very well for the distinction between musical works and their interpretations.

These accounts run the risk of rendering the medium of the work irrelevant to its understanding or appreciation. Yet it is an open question in aesthetics whether there are genuinely relevant aesthetic differences between, say, concert performances of Jupiter and playings of a recording (for one viewpoint see Mag Uidhir 2007; for another, Davies 2001, chapter 7).

As ideas, musical works would pop in and out of existence as people (or the relevant people) thought of them. They would constitute whatever it is that ideas constitute (the neural correlates of mental states). But while people surely have ideas of and ideas about musical works, the works themselves are not identical with those ideas.

\section{Summary}

1. Insofar as these novel accounts capture the musical work concept, they point to a metaphysically dubious category (e.g., abstract particulars). Debate continues about these categories. And insofar as these accounts point to a plausible category, they do not capture the musical work concept. They might be describing something - the generative act of musical works, the ideas of musical works-but to consider these things as musical works is to require much unmotivated revision of the concept under scrutiny.

2. Some of the more revisionary theories introduce a new concept to "pick up" what is usually captured by the ordinary musical work concept. For example, David

\footnotetext{
${ }^{47}$ Although, not for Collingwood: instead, the work is the idea of the composer, and the audience's job is to recreate that idea in their own minds.
} 
Davies' works-as-action-tokens account introduces the concept of the work-focus. Yet, even if we were to follow his lead on this, the ontological status of the workfocus remains elusive, and after all it is now that concept that I am explicating here (in the context of his framework/terminology) by explicating the target of the musical work platitudes.

\subsection{Conclusion}

Aside from the specific objections to each account, the theories on offer in general either necessitate unwarranted conceptual revision (indeed some of them change the target of analysis completely), or acquiesce in suspicious metaphysical commitments. In my view, this situation calls for an alternative account. Is there a theory that can account for how musical works are conceived - that is, plays the role specified by the musical work platitudeswithout positing dubious ontological categories? Moreover, is there such a theory that is plainly compatible with a philosophically naturalistic perspective? A theory that does not conjecture into existence entities that cannot in principle be empirically confirmed? Kania $(2008,2012 a)$ puts one such account on the table: fictionalism. ${ }^{48}$

Interpreting the musical work concept in a fictionalistic light is one underexplored and underappreciated option. ${ }^{49}$ On this view, talk of musical works is, in David Davies' words, something like 'a useful fiction that allows us to talk about those ways of grouping performances that interest us' (D. Davies 2009, p. 745). The idea is that although we all recognise the concept of musical works, and that we conceptualise musical works as being created by their composers, exemplified in their performances, and so on-and, moreover, that that's because there is a shared, intersubjective system of representations of musical

\footnotetext{
${ }^{48}$ Martin (1993) proposes that talk of musical works could be construed as a fiction that gives way to a more convenient discourse. (Ultimately he opts for a realist, type/token view.) Kania (2008) does not endorse fictionalism either, but explores it as a viable option. Kania (2012a) revisits the idea more explicitly, arguing that fictionalism is preferable to Platonism about musical works, although he still falls short of a full endorsement. Goehr could be interpreted as a fictionalist: she thinks of musical works has having 'projected' or 'fictional' existence (Goehr 2007, p. 106). But she does not elucidate this aspect of her view; her project is to explicate, primarily historically, the musical work concept. ${ }^{49}$ Davies (2001) quickly rejects fictionalism about musical works (pp. 39-40), however the version of fictionalism he rejects treats statements about musical works as statements about performances. I defend a more developed fictionalist account herein, which is not subject to Davies' criticisms.
} 
works - they need not be more than fictional objects: intentional inexistents. This possibility does not require the positing of metaphysically suspicious or independently unmotivated entities (irksome to many philosophical naturalists), yet it will allow for enough conceptual retention of the musical work concept and platitudes (unlike the too-revisionary accounts which require conceptual revision) even though some propositions ordinarily believed to be true will turn out, strictly speaking, false. But I take this as a small price to pay, of no concern to the participants of the target Western art music tradition. I develop this view in the following section.

\section{Fictionalism about musical works}

Fictionalism is a theory always applied to some subject matter; it interprets thought and talk about that subject matter as a useful fiction. Philosophers have developed a number of fictionalist theories, such as fictionalism about fictional characters (Brock 2002, 2016), fictionalism about mathematical entities (Field 1980, 1989; Balaguer 2009), and fictionalism about possible worlds (Rosen 1990). Each case for fictionalism is to be assessed on its own merits. Here I develop fictionalism about musical works. ${ }^{50}$

Fictionalism resolves an ontological puzzle; it is a way out of the infamous Quinean trilemma, according to which there are just three ways to deal with statements that seem to commit speakers to the existence of an unwanted entity (see Quine 1960). First option: change one's linguistic expressions_-paraphrase the apparent commitment away (for instance, instead of saying "unicorns have horns", explicitly say "according to the myths, unicorns have horns" or "I conceive of unicorns as horned"). Second option: stop talking about those things completely. Third option: give up and accept the existence of the controversial entity. Sometimes none of these options appeal, and it is my contention that sometimes this is the case for musical works. Fictionalism is a fourth option unidentified by Quine. It is a theory by which one can disclaim unwanted ontological commitments to suspicious or dubious entities, whilst reaping some of the benefits of those very

\footnotetext{
${ }^{50}$ Given philosophers' preoccupation with the ontology of musical works (and the debate's primary place in the philosophy of music literature), it is worth developing a plausible fictionalist position somewhat independently of explicit debate about realism/anti-realism about musical works. Without it, theorists might be tempted to dismiss "straw man" fictionalist positions, potentially neglecting a useful linguistic and conceptual framework and a metaphysical possibility worth entertaining.
} 
commitments, not through paraphrase but by putting forward statements in a fictionalistic light. So as long as the fictionalist has a claim to the fiction's utility, fictionalism is having one's cake and eating it too.

The advantages of a fictionalist rendering of the subject matter are thus considerable. Although the fictionalist is not committed to the existence of some controversial ontological category, the fictionalist can continue to reason with respect to it about salient empirical matters, can continue to generate predictions/generalisations, and can continue to make critical/evaluative claims. In this section, I motivate and develop fictionalism about musical works. I begin my fictionalist proposal by suggesting that there is no good reason to posit the existence of musical works. And, throughout, my strategy will be to develop my view through a critical discussion of the surrounding philosophical research.

\subsection{Interlude: intentional inexistents}

I take the preceding discussion to motivate my providing of an alternative account to the standard (realist) views of musical works. My account is anti-realist, rendering musical works as fictional. Now, of course, we think and talk about musical works. That is, the logical status of musical works as (mere) objects of intentionality is not what is at stakeafter all, we can straightforwardly think and talk about things that do not exist: our mental states and our utterances can be directed at the non-existent (Crane 2014) - though in this section I will explain the move to call them intentional inexistents.

Roman Ingarden (e.g., 1989) argues that musical works are intentional existents: 'a purely intentional object which has its source of being in the creative acts of the composer and its ontic foundation in the score' (Ingarden 1989, pp. 90-91). So, accordingly, a musical work is an object that can be thought about, is entirely mind-dependent (although, for Ingarden, that it exists is linked to a composer's acts and a score), but is not reduced to the idea of the musical work.

I think Ingarden was almost on the right track. However, in my view, objects with only "intentional existence", as cashed out by Ingarden, have no real existence at all. We can think and talk about phlogiston, unicorns, or miasmic vapours yet these things are not 
real. Ingarden admits this himself, when he states that 'the musical work is a purely intentional and not a real object' (Ingarden 1989, p. 93). And I do not see any reason to follow Meinong (1904) in distinguishing the real from the existent. In my view, we are all better off saying "intentional inexistents": we conceive of, and think and talk about these "things", but they do not exist. They are fictional. It is pointless to say that mythological beings or discredited theoretical posits "exist" in a "purely intentional" sense. ${ }^{51}$ Again, in my view, that is just to say that they have no real existence at all. ${ }^{52}$ Anything could be the subject of thought or talk — consider round squares, or the three actual-apples-that-I-amcurrently-(merely)-imaginging — and admitting such entities into an ontological theory leads to very dubious metaphysics. Just thinking or talking about actual-apples-that-l-amcurrently-(merely)-imaginging does not create any actual apples (Caplan 2004). Same goes for round squares. And, I contend, the same goes for the likes of The Jupiter.

Following Georges Rey's (2006) claim that standard linguistic entities—words, sentences, and so on-are intentional inexistents, Kania (2008) asks whether musical works might be the same. According to this proposal about musical works:

(i) there are no such things, either outside the mind or in the concrete or abstract realms, or inside the mind, but that (ii) there is a quite robust shared system of representations of such things. (Kania 2008, p. 439.)

\footnotetext{
${ }^{51}$ Bertrand Russell puts the point vehemently: 'many logicians have been driven to the conclusion that there are unreal objects... In such theories, it seems to me, there is a failure of that feeling for reality which ought to be preserved even in the most abstract studies. Logic, I should maintain, must no more admit a unicorn than zoology can; for logic is concerned with the real world just as truly as zoology, though with its more abstract and general features. To say that unicorns have an existence in heraldry, or in literature, or in imagination, is a most pitiful and paltry evasion... What exists is a picture, or a description in words... The sense of reality is vital in logic, and whoever juggles with it by pretending that Hamlet has another kind of reality is doing a disservice to thought. A robust sense of reality is very necessary in framing a correct analysis of propositions about unicorns... and other such pseudo-objects' (Russell 1919, pp. 169-170).

${ }^{22}$ Ockham's Razor, at least, provides well-principled motivation to cull such intentional inexistents from our ontological commitments. The argument in short (following Brock 2015): all things being equal, we are justified in adding to our ontology if there is some explanatory value in doing so. But there is no explanatory value in including merely intentional objects in our ontology. (At least, the burden of proof is on the realist about merely intentional objects. After all, they are the ones positing unfalsifiable, or at least, suspect, entities.) So, all things being equal, we are not justified in including merely intentional objects in our ontology. That is, a more parsimonious account is to be preferred.
} 
The idea is that those of us who wield the musical work concept acquiesce in a fiction. It can be true that a person is thinking about The Jupiter even though, on this view, The Jupiter is not real. It is an error theory of musical works. Kania (2008) leaves fictionalism about musical works underdeveloped —and Letts (2015) points out that Kania's account is found wanting. ${ }^{53}$ I further develop my view in what follows shortly.

So I think that by developing an account of musical works as intentional inexistents I can save the musical work concept platitudes. It makes no difference to ordinary discourse, nor to artistic practice, if musical works are intentional inexistents, provided we all continue to act and talk in the same way, thinking and speaking as if there were such things. We do not have to revise our concept of musical works. A person newly-converted to my view can still apply the musical work concept in the same manner and continue to speak in the same way (though in a "fictional spirit"), since doing so will satisfy all of her conversational aims.

To see my point, imagine that I want to talk to you about happiness, and I want to convey to you my observation that my friend (the man standing over there with a glass of clear liquid) is happy. So I say "The man holding a glass of water is happy". When you retort that he is holding a glass that actually contains vodka, I will become impatient (Eklund 2005). It's the man's happiness I want to talk about; whether his glass contains water or vodka is beside the point. So likewise, for example, when I claim that "Last night's performance of The Jupiter was beautiful", I will become impatient when you reply there is no such thing as The Jupiter. It was that performance or that occurrence of music that I wanted to talk about. And that exists. I just needed, somehow, to make it obvious to you which musical occurrence I was talking about. Saying it the way that I did is a convenience. The ontological commitments entailed by the proposition semantically expressed by my utterance are—for present purposes—beside the point. ${ }^{54}$

\footnotetext{
${ }^{53}$ Letts is critical of Kania's fictionalist approach, but thinks that a "material fictionalist" approach might be plausible. I take my account to be generally compatible with Letts' preferred variant (in ways that should become clear along the way) but I do not cash it out in his terms, and I will be unconcerned with Letts' critique of Kania's specific contentions.

${ }^{54}$ This is not to suggest that there are no challenges to interpreting fictionalist thought and talk. My specific brand of fictionalism is developed throughout the next sub-section.
} 
In sum, then, I do not think that the musical work concept needs to be revised. We just do not need to count musical works in our ontology, as we do not count unicorns. And although I suggest we need not count musical works in our ontology, I nevertheless suggest we can continue to speak of them as a kind of useful fiction.

\subsection{Revolutionary fictionalism about musical works}

In my view, there are no musical works. (More specifically: postulating them into existence seems to me unmotivated and unnecessary.) This is based, in part, upon the state of play: accepting a realist theory either means positing a metaphysically suspicious category or a revisionary conception of the concept under scrutiny. As I have argued above, neither option appears to have much to recommend it.

Yet we think and talk as if musical works exist. The plausibility of thinking that there are musical works is explained by the fact that there really are displays. And some of what we say or think about musical works is true, or approximately true of displays. But not all. The remainder can be coherently interpreted by the fictionalist account that I develop herein. That is, some of the advantages that we would hope to get from talking or thinking about musical works do not genuinely depend on musical works being real things, or even on our believing that they are real things. They are convenient fictions. It is time now to unpack fictionalism.

There are two theses associated with fictionalism—a linguistic thesis, and an ontological thesis. The linguistic thesis maintains that 'utterances of sentences of the discourse are best seen not as efforts to say what is literally true, but as useful fictions of some sort' (Eklund 2011, §2.1). The ontological thesis is anti-realist, rejecting the existence of the entities associated with the concept at hand.

Following a distinction made by Burgess and Rosen (1997), philosophers distinguish hermeneutic fictionalism from revolutionary fictionalism. Hermeneutic fictionalism has been advocated about fictional characters. It is plausible that people are already fictionalists about fictional characters: they do not believe that Sherlock Holmes exists, but continue to say things like "Sherlock Holmes lives on Baker Street". Two proposals about such statements have been given: that people either engage in make-believe in fictional 
character discourse (pretence hermeneutic fictionalism—see Walton 1990, 2005) or short-hand speak which contains tacit constituents (prefix hermeneutic fictionalism—see Brock 2002, 2016).

On the other hand, revolutionary fictionalism prescribes a reform, not about the target concept, but the wider conversational context as analysed philosophically. Revolutionary fictionalism has been advanced with respect to moral theory (Joyce 2005) and mathematics (Field 1980). I will advance it for musical works. ${ }^{55}$

Take Field's mathematical fictionalism. Despite philosophers like Quine and Putnam famously maintaining that mathematics is indispensable to science (for review see Colyvan 2015), Field does not think that the entities postulated by mathematical theory, such as numbers, exist at all. But he agrees that the conventions and discourse of mathematics allow people to realise important information about things that do exist, so it is a valuable discipline. Field acknowledges mathematics, then, as a useful kind of fiction, but not as a domain of truth, strictly speaking, at least insofar as mathematical statements are interpreted at face-value. His fictionalism is revolutionary: a kind of error theory about numbers, but without abandoning the discourse. And notice that it does not require conceptual revision: our concept of number, for instance, is not modified in the process.

However, philosophical analysis of statements about the targets of a fictionalist account is somewhat modified in light of fictionalism, since a face-value interpretation of those statements ignores the fictionalist's linguistic thesis. There is a typical tripartite distinction between such statement kinds (following Brock 2002, see p. 4). I distinguish them as such:

(1) Fictional statements internal to the relevant fiction. For example, the fictionalist about musical works might utter:

(IS) "The Jupiter was composed by Mozart, completed in 1788."

\footnotetext{
${ }^{55}$ Notice that revolutionary fictionalism is compatible with a descriptive-leaning metaphysics: the revolutionary fictionalist need not revise the musical work concept. The "revolution" is purely one of ontological commitment and wider philosophical context.
} 
(2) Fictional statements external to the relevant fiction, although analysed in virtue of a fictional context. These include critical and evaluative claims. For example, a fictionalist about musical works might utter:

(ES) "The Jupiter is more beautiful than a snow-capped mountain."

(3) Non-fictional statements that express one's ontological commitments. For example, a fictionalist about musical works might utter:

(OS) "The Jupiter does not exist."

Consider the last of these first. As an ontological statement, (OS) can be straightforwardly interpreted, at face value, in line with the fictionalist's ontological commitments. We deal with these non-fictional statements as we deal with ordinary non-existence claims such as "There are no unicorns" and "phlogiston does not exist". The fictionalist uttering (OS) does not intend their utterance to be, nor should it be analysed as, indexed to a fictional context; it is a statement put forward that is divorced from any relevant fiction. On the other hand, (IS) and (ES) statements require further analysis.

\subsubsection{Fictional statements internal to the musical works fiction}

Consider first internal statements, such as (IS). I think (IS) is best conceived as a statement that purports to capture something about the content of the Western classical art musical tradition/practice/discourse - qua "fiction"-insofar as it coheres with empirical facts about the way the world is. (That is, I shall advocate an object-fictionalist theory, but I shall return to this distinction later.) In other words, (IS) is a statement about the empirical facts (pertaining to Mozart's creative activity around 1788) that "conceptually intersect" with an intentional inexistent, within the context of the tradition/practice/discourse in which the concept is legitimised. That is to say, colloquially, musical works are the fictional characters in the Western classical art music fiction. 
Since according to that fiction (IS) is true, we can utter (IS), albeit "fictitiously", with a genuine sense of conviction. Balaguer (2009) would say that (IS) is "fictionalistically correct"; Sainsbury (2010) would say that (IS) maintains "fidelity" to the relevant "fiction". In contrast, consider statements like "The Jupiter was composed by Beethoven, completed in 1788", or "The Jupiter was composed by Mozart, completed in 1890 ". Not only are these statements false at face value, they are false according to our fiction. So they are not "fictionalistically correct"; they do not maintain "fidelity". ${ }^{56}$

But what does putting a statement forward fictionally — that is, what does "fictionalistic truth" discourse-really amount to? Answering this requires some unpacking.

As mentioned above, philosophers distinguish prefix fictionalism from pretence fictionalism. Pretence theorists, in a revolutionary fictionalism context, argue that sentences about the entities in question should not be asserted, but put forward through some other speech act, such as pretence or make-believe, removing the assertive force of these sentences (Kroon 2011). Prefix theorists, on the other hand, suggest that the sentences be asserted, but with a tacit-constituent-a prefix such as "According to the Sherlock Holmes stories", or whatever-thus putting the sentences forward as short-hand talk for longer sentences beginning with a context-operator. The full, compound sentence might be true, in spite of the falsity of the embedded sentence (taken simply at face-value).

Admittedly, the pretence/prefix distinction may make more of a difference in hermeneutic fictionalism cases. That's because whether pretence or prefix fictionalism actually holds matters for the theory's plausible descriptive application (though see Howell 2015 for caution). In revolutionary cases, the difference is mainly pragmatic: the theorist recommends the one (or blend of both) that they think best suits the ongoing discourse once reconceived as fictionalistic.

\footnotetext{
${ }^{56}$ Consider, as Field (1989) does, the difference between "Oliver Twist grew up in London" and "Oliver Twist grew up in L.A." The former is true according to the story, the latter is not. Yet both are false simpliciter for the fictionalist about fictional characters who believes that there is no Oliver Twist. And, similarly, as Daly explains, 'It seems correct to say that Pegasus was a winged horse... and incorrect to say that Pegasus was a scuba-diving pig... 'Pegasus is a flying horse' is correct only in the sense that 'Pegasus is a flying horse' is true-in-Greek-legend. "Pegasus is a flying horse' is truein-Greek-legend' is itself true if and only if 'Pegasus is a flying horse' is contained in, or is a consequence of... the Greek legends' (Daly 2008, p. 426). That is, Pegasus being a scuba-diving pig is obviously not a part of those legends.
} 
So there are two broad models - two façons de parler — by which a revolutionary fictionalist can construe fictional discourse. Either, or some mix of both, is ultimately consistent with my basic line. Here I (briefly) explain why I lean towards the prefix model.

Kania (2012a) thinks that fictionalists about musical works should endorse revolutionary fictionalism, and that according to the fictionalist, "What would in the past have been assertions about musical works, for instance, ought really, according to the fictionalist, to be put forward as make-believe' (Kania 2012a, p. 215). I agree with him that ficitionalists about musical works should apply a revolutionary (versus hermeneutic) brand of fictionalism. That's because I presume that people tend not to already disbelieve in the existence of music works (compare with fictional characters: people tend to already disbelieve in the existence of, say, Sherlock Holmes). Kania's recommendation of pretence is one place where I depart from his view.

The differences between prefix and pretence fictionalism are, in fact, quite substantial. The pretence theorist claims that (i) utterances of (IS) are not truth-normed, but have some other virtue; (ii) utterances of (IS) should be taken at face value; (iii) utterances of (IS) are truth-apt, yet never true; (iv) utterances of (IS) should not be believed but merely be accepted/pretended/make-believed; (v) utterances of (IS) do not assert—rather, some other speech act is taking place.

The prefix theorist, on the other hand, claims that although utterances of (IS) taken at face value are false, strictly speaking, they really can be true according to a specific context, story, theory, or practice. So the prefix theorist suggests that utterances of (IS), when put forward seriously/genuinely, are (i) truth-normed and (ii) short for an extended sentence beginning with a contextual operator. In long form, those sentences (iii) are truth-apt, (iv) believed by the speaker, and (v) really asserted.

I am hesitant to prefer pretence fictionalism in the context of musical works for several reasons. However, I will be brief on this matter. Firstly, some legitimate participants in the practice/discourse may not be able to pretend or make-believe in this way (some autistic individuals, for instance; see Stanley 2001). Secondly, in light of recent semanticdescent models and models of non-conscious assumptions of ordinary agents, ascribing pretence or make-believe in a context like this may well be implausible (Howell 2015). 
Thirdly, implying that serious research going on in, say, music departments ought to be under a guise of pretence or make-believe, rather than simply involving fictional entities, might be uncharitable and demeaning to good research done, stunting progress. While pretence fictionalism is intuitively a good description of the way in which adults interact and engage with young children, say, in the context of the Santa Claus mythology, it is not a good fit for the musical work discourse. ${ }^{57}$

So, on my view (that is, a revolutionary, prefix-oriented fictionalism about musical works), internal fictional statements can still be interpreted as asserted, true, and believed, as long as they are conceived as elliptical for statements with a context-operator. Consider talk of fictional characters. We can, for example, coherently assert that "Sherlock Holmes does not exist", and then that "Sherlock Holmes is a detective living on Baker Street", if the latter is asserted in a fictionalistic light. It is short-hand for something like "According to the Sherlock Holmes stories, Sherlock Holmes is a detective living on Baker Street". Thus the shorter sentence "Sherlock Holmes is a detective living on Baker Street" is, fictionalistically (that is, prefixedly), true. In my theory, the Western art musical tradition/practice/discourse plays the role of the relevant fiction, about which there are many empirical truths, while the musical works play the role of fictional characters embedded in that "fiction".

A quick point of clarification might be useful, about the exact nature of my intended musical work context-operator. Yablo (2001) distinguishes meta-fictionalist operators ("according to the fiction/the fiction is such that, $\mathrm{S}$ ") from object-fictionalist operators ("according to the world/the world is such that, S"). The "according to the Sherlock Holmes stories" operator, discussed above, is meta-fictionalist: when a prefix-fictionalist about fictional characters asserts "Sherlock Holmes is a detective living on Baker Street", she is merely concerned with the content of a fiction (Brock 2016). The object-fictionalist operator, on the other hand, is concerned with how the world is, with respect to the content of the relevant fiction. So in uttering $\mathrm{S}$, the object-fictionalist is asserting that the world is such (e.g., that all the necessary and sufficient conditions are in place for it to be true) that according to the relevant fiction, S. Take fictionalism about mathematical entities

\footnotetext{
${ }^{57}$ Consider, 'Most of us stop believing in Santa Claus at some point, but many of us replace our belief in Santa Claus with an elaborate pretense involving imagining him coming down chimneys and living at the North Pole. This seems quite unobjectionable, presumably in part because nothing too serious is at stake' (Hussain 2010, p. 339).
} 
as an example. When the object-fictionalist declares that "the number of Martian moons is two", she appeals to the worldly facts that there is a Martian moon, $x$, called "Phobos" and a Martian moon, $y$, called "Deimos", and that $x$ and $y$ are different from each other, and that nothing else is a Martian moon, in order to make her statement true according to the number fiction. She does not merely appeal to the content of standard mathematics.

Given the distinction, then, which way should my context-operator be intended? In my view, object-fictionalism of musical works is to be preferred here. First, the Western art music tradition, although widely discussed, catalogued in many books, and so on, is not articulated and orally preserved, or written down and published, in the same sense as a myth or Sherlock Holmes story. Rather, from the Western art music tradition a communitybased fiction (Currie 1990) has emerged. And there are many truth-apt things to be said about that tradition/community.

Second, although according to the Western art music tradition The Jupiter was composed by Mozart and completed in 1788 , it could be that Mozart actually did what he did in 1786, and that we just have it wrong. This would be an empirical matter, turning on the timing of the creative acts of Mozart. Thus we should not think that the truth of our long-form statements turns simply on the "content" of the "fiction", but the way that the world really is.

So the context-operator, in my view, is best intended as something like "the world is such that (that is, given that it contains the Western art music tradition, we can coherently say), S". After all, the relevant tradition/practice/discourse (the so-called "fiction") is not itself a fictional thing: the musical work tradition/community is a matter of social ontology.

That is, to say fictionalistically "The Jupiter was composed by Mozart and completed in 1788 " is to speak in short for something like "the world is such that we can coherently say The Jupiter was composed by Mozart and completed in 1788"- - that is, "because the world contains that tradition and according to that tradition, the musical work in question was composed by Mozart and completed in 1788, we can say (since it will satisfy our conversational aims) that The Jupiter was composed by Mozart and completed in 1788". This is not as convoluted as it may appear. The idea: the world contains a tradition, and this 
tradition tells us there is an intentional object The Jupiter and as such we can think and talk about that intentional object, meaningfully and coherently, without strictly speaking referring. We just need not include it in our ontology.

Third, meta-fictionalism cannot seem to get at the heart of some of the things that people really care about. When a fictionalist about mathematical entities laments that the numbers of the endangered species $E$ are diminishing, she is not sad about some fact about the content of a fiction; she is sad about the plight of real entities. Similarly, the musical work discourse is useful for discussing things many people really care about: Western art music as well as various aspects of it..$^{8}$

\subsubsection{Fictional statements external to the musical works fiction}

External statements require a slightly different analysis to internal statements. Consider again (ES), "The Jupiter is more beautiful than a snow-capped mountain". I shall specify that this is a critical/evaluative claim, external to any such fictional context: aesthetic evaluation is a matter for critical debate, not straightforwardly specified by the existence of the Western art musical tradition/community. Yet, at first glance, in order to take this statement seriously it seems as though a real relation must be posited, more beautiful than, that holds between (real) relata! In external statements, unlike internal statements, it appears that the musical works themselves are the things that are represented. Is there an elegant, fictionalist answer for such a challenge?

I think so. My answer has two parts. First, sometimes external statements are put forward in a nominalist light. This is a descriptive, empirical claim. A statement like "I really enjoyed The Jupiter" could have been—either upon reflection, or off-the-cuff-put forward by the speaker about a particular musical performance, perhaps last night's. Here the speaker intends to convey to her interlocutors something like "I really enjoyed the performance of music last night called 'The Jupiter"'. This is the sort of eliminativist

\footnotetext{
${ }^{58}$ There is one standard objection to applications of object-fictionalism: that it cannot account for reflexive cases (Yablo 2001 calls this objection "The Bomb"). For example, the object-fictionalist about mathematical entities struggles to deal with "the number of numbers", "How many numbers are there?", and so on; similarly, the modal object-fictionalist struggles with "the possibility of possibilities", and the like. I take it that the musical work application of object-fictionalism is not vulnerable to this objection. "The musical work of musical works" is hardly a coherent notion.
} 
paraphrase advocated by Rudner (1950). If this is ever actually the case, the statement is already put forward in a way that is consistent with fictionalism's ontological commitments, so no additional explanation is required. (And one does not need to be a fictionalist to accept this!) In this scenario, the apparent commitment to a problematic entity is "paraphrased away", one of Quine's three strategies. Likewise, sometimes external statements are put forward in an expressivist light. Again, this is a descriptive, empirical claim. A given utterance of (ES) might merely be expressive of its speaker's aesthetic attitudes and preferences, and not intended as truth-apt, or even "about" anything other than the fact that the speaker conceives of The Jupiter as thus-and-so. No tacit constituents of sentences (or pretence) are called for in analysing statements intended on some occasion of use to be like this.

However, descriptively, it is not at all plausible to claim that these scenarios are always the case. For example, sometimes external statements are intended by speakers to be about something that is "in addition" to the various reductions (score-copies, performances, and so on). Rudner's example...

"Beethoven's Fifth Symphony is good but this is a bad rendition of it" could be taken as an ellipsis for "there is a musical rendition called Beethoven's Fifth Symphony which is pleasing esthetically but this musical rendition, while similar to it in important respects, is esthetically displeasing" (Rudner 1950, p. 385)

... is not at all convincing. In much musical critical discourse, speakers put forward statements that ought to be interpreted as aiming at a given musical work-that is, put forward with the musical work concept in mind—not "really" about some particular performance. Suppose that a musical work has only been given one performance, and suppose further that it was poorly performed. In this case, there is no particular rendition to which the speaker could attempt to refer when she says "It is a good piece, but that was a poor performance of it". 
My recommendation is to revise, at least in philosophical discourse, external talk of musical works. When at all possible, critical/evaluative talk should be about displays. In my view, we would all speak much more clearly if we said that (for example) "that performance called 'The Jupiter' was more beautiful than a snow-capped mountain”, or that "competent The Jupiter performances are more beautiful than a snow-capped mountain", or that "my imagined The Jupiter rendition (from reading a score-copy) was more beautiful than a snow-capped mountain" and so on. After all, these are the things that have aesthetic properties that we are able to experience, enabling our aesthetic evaluations, so critical/evaluative talk of musical works is, strictly speaking, dispensable given fine-grained enough talk about concreta. However, this kind of talk is incredibly cumbersome for general usage. So my recommendation is to treat general occurrences of the name "The Jupiter" (and its cognates) as syncategorematic (Quine 1939).

Syncategorematic terms are meaningful terms that do not designate anything. They allow us to talk of inexistents, in this case musical works, as a useful fiction. (And just as we can easily and uncontroversially distinguish other inexistents-we can distinguish talk of phlogiston from talk of miasmic vapours, Vulcan, Santa Claus and so on-we can straightforwardly distinguish talk of The Jupiter from Beethoven's Fifth Symphony.)

This is somewhat reminiscent of Jeremy Bentham's account of fictional entities. Bentham distinguishes fictitious entities from real entities, yet he notes that fictitious entities cannot have the property of being fictitious since they do not exist to have any properties at all. Alleged fictitious entities are such 'which, though by the grammatical form of the discourse employed in speaking of it, existence be ascribed, yet in truth and reality existence is not meant to be ascribed' (Bentham 1932, p. 12). Bentham turns instead to names of fictitious entities. The idea is that instead of saying that the golden mountain is fictional, one says that "The golden mountain" is a fictitious-name: 'a name whose use is not intended to convey to the hearer a commitment to the real existence of an object corresponding to it' (Rosen 2005, p. 51). It is my contention that “The Jupiter is more beautiful than a snow-capped mountain" is best put forward as elliptical for something like “'The Jupiter' is a fictitious-name for an intentional inexistent more beautiful than a snowcapped mountain" (or similar). And the plausibility of thinking that the intentional inexistent is somehow more beautiful than a snow-capped mountain is explained by 
thinking that the relevant displays are so, or at least typically so. We best think of the situation in the following way. The fictional-name, "The Jupiter", "here purports to designate some one specific entity' (Quine 1939, p. 701); that is, 'the word is not a name of any entity in its own right... it is a noun at all only because of a regrettable strain of realism which pervades our own particular language... The mere capacity to turn up in a sentence does not make a string of marks a name' (Quine 1939, p. 704). So we need not forego the convenience of external statements like "The Jupiter is more beautiful than a snow-capped mountain"; their usage can be a mere/short-hand façon de parler, not committing anyone to the belief in musical works. Real entities in the vicinity of musical work talk can be said, sensibly, to be more beautiful than a snow-capped mountain, or otherwise (e.g., a particular performance, an imagined performance through reading a score, a mental representation of typical features of competent performances going by that name and with the relevant causal history, and so on).

Balaguer (2009) calls this strategy “theft-over-honest-toil” fictionalism (though not in Bertand Russell's famously pejorative sense of that phrase, since it does not posit any suspicious entities). Advocating this form of fictionalism about mathematical entities over the Platonist alternative, Balaguer writes that a given statement involving apparent quantification over mathematical entities (e.g., "There are fewer Martian moons than Neptunian moons" or "There are at least two numbers") can still be put forward as true (that is, it is "fictionalistically" true), 'iff it would have been true if there had actually existed abstract mathematical objects of the kinds that platonists have in mind, i.e., the kinds that our mathematical theories purport to be about' (Balaguer 2009, p. 138). Stuart Brock (2002, 2016) thinks of this "parasitic" strategy as "according to the realist's hypothesis"fictionalism. A statement such as "Sherlock Holmes is more famous than any living detective" can be put forward as elliptical for "According to the realist's hypothesis about fictional characters, Sherlock Holmes is [such that he is] more famous than any living detective".

Imagine a straightforwardly-descriptivist musical work realist that simply posits a sui generis category of entities that realise the platitudes systematised by the Canberra Plan method. Call this realist a "brute-realist". I contend that any proposal that the brute-realist can give for the truth of an external statement can be stolen by the revolutionary 
fictionalist—in the name of the revolution!- -and used in an argument for that very statement's "fictionalistic" truth, or truth according to some tacit context-operator. Then, "The Jupiter is more beautiful than a snow-capped mountain" can simply be elliptical for "According to the brute-realist's hypothesis about musical works, The Jupiter is [such that it is] more beautiful than a snow-capped mountain". Moreover, my fictionalist theory retains an advantage over the brute-realist's theory: I do not posit any suspicious or dubious entities, and yet anything the brute-realist says to her merit, I can simply commandeer as fictionalistically true. The musical work platitudes come out fictionalistically true, so no conceptual revision is necessitated. Statements identifying musical works (e.g., "The Jupiter is a musical work") come out false when taken at face value, but prefixedly true (that is, fictionalistically true), identifying something about the world (that the Western art music tradition qua fiction contains a fictional character, The Jupiter). And in my view, prefixed-truth leading to real truths is better than no truth at all, or massive revision, or metaphysically dubious commitments. When those are the other options, I think my brand of fictionalism wins the stalemate.

\subsubsection{The incredulous stare!}

In sum, then, my proposal is as follows. Explicit ontological statements concerning musical works are to be treated literally, at face value. Internal statements concerning musical works should be treated as fictional statements that purport to cohere with how the world is, given that it contains the Western art music tradition, and that from this tradition a kind of community-based "fiction" has emerged, according to which it can be said that thusand-so. External statements concerning musical works should be about displays, whenever possible, but when a nominalisation or other such strategy is undesirable, they should be treated as fictional statements about the musical-work posits of the brute-realist.

So there is a uniform, principled fictionalist treatment available for all three statement kinds. In my view, the benefits of fictionalism about musical works are great; the costs are slight.

However, as with any philosophical theory, there is going to be dissent about revolutionary fictionalism. According to John Burgess, revolutionary fictionalism is 
laughable-'comically immodest' (Burgess 2004, p. 30). ${ }^{59}$ Burgess's incredulous stare was originally directed at mathematical fictionalism, but it can be seen how it might be applied to the case of musical works. ${ }^{60}$ The idea: the professional participants of the Western art music practice are the experts when it comes to musical works (just as mathematicians are in the case of mathematics), so why is a philosopher trying to butt in and tell them that, "technically speaking", some of their sentences are face-value wrong and their utterances are best put forward in a fictional spirit?

This worry can be easily mitigated. My fictionalist revolution is artistically/creatively (and perhaps even musicologically) uninteresting and unimportant. It matters not for the participants of the practice if musical works are the fictional characters of the Western art music fiction. It is not something that participants in the practice think or care about (except philosophically - it is not relevant, musically, to the practice), and the philosophical merits of fictionalism (or any other ontological theories about musical works for that matter) is something that philosophers are the experts in, not the participants of the practice (except insofar as they don their philosopher's hats). To be sure, the point that the fictionalist is making 'is a paradigmatically philosophical point' (Balaguer 2009, p. 154). As Mary Leng notes, 'Revolutionary fictionalism need not advocate a revolution in practice, only in our understanding of that practice' (Leng 2005, p. 278).

\section{Conclusion}

So, in my view, we should be fictionalists about musical works. This does not require revising artistic practice, or the musical work concept. It hardly requires revision of musical discourse at all; just the wider conversational context, philosophically speaking. And this is a small price to pay for an otherwise naturalistically compatible and ontologically au courant theory. My suggestion is in two parts. First, that internal fictional statements about musical works be put forward in an object-fictionalist light; that is, put forward as short for statements about the world that are true (or not) in light of facts about the Western art music tradition/community. On this view, to say that "The Jupiter was completed by Mozart

\footnotetext{
${ }^{59}$ This kind of objection was put to anti-realist theories more generally by Lewis (1986).

${ }^{60}$ Burgess also claims that revolutionary fictionalism commits an error against Carnap. But evaluating Carnap's philosophy and its consequences will take me too far afield and is not central to my concerns here.
} 
in 1788" should be convenient, every-day short-hand for the lengthy "the world is such that (that is, given that it contains the Western art music tradition, we can coherently say), The Jupiter was completed by Mozart in 1788". Second, that external fictional statements about musical works, when not put forward in a nominalist light (Rudner 1950) or eliminated for the purposes of clearer critical/evaluative talk of concreta, be put forward as if musical works existed; that is, put forward according to the brute-realist's hypothesis. The use of the name "The Jupiter" is best conceived as syncategorematic in these statements.

In this chapter I have brought my pluralistic, naturalistic perspective about music generally to bear on the Western art musical work concept-a specific univocal concept, emerging from the Western art music practice (Goehr 2007). I have argued that the ontology of musical works best lends itself to a descriptivist-sided methodology, and I have championed the Canberra Plan. The Canberra Plan takes seriously the platitudes from the relevant practice/discourse that ground the relevant concept. Once this is acknowledged, fictionalism about musical works becomes a viable contender. Kania (2008, 2012a) suggests that it is a plausible option to take, but leaves the proposal underdeveloped. I have developed it here. Unlike the (realist) ontological accounts of musical works already on offer, fictionalism does not posit the existence of dubious or suspicious metaphysical entities, and nor does it revise the concept under scrutiny. We may speak as if there are musical works, but strictly speaking, we fail to refer to any such works (a small bullet to bite). I have explained how our statements about musical works can nevertheless be retained as truth-apt, by appealing to the tacit constituents that we can intend our statements to contain, when we are speaking of musical works as a useful fiction. 


\section{Chapter 6: Evolutionary status of music}

Let's recap. I have motivated and defended my polysemy analysis of sound and conceptual pluralism about music. Then, with a relational conception of music in mind (specifically, music tied to the Western art music tradition/discourse/practice), I zoomed in on a specific work concept: I motivated and developed a fictionalist account of the category specified by the Western classical musical artwork concept. In this chapter, I zoom out. Far out. I will be concerned with the recent interdisciplinary discussion that philosophers have been contributing to (see e.g., Davies 2012b; Dutton 2009) about the evolution of music. That discussion is largely couched in the adaptation/by-product framework. Advocates of an adaptationist view search for a proper function, in the biologist's sense, that music may have plausibly played in ancestral hominin environments, contributing to the survival/ reproduction of individuals, or perhaps groups, comprising our ancestral lineage. Additionally, theorists have defended various other views: that music is a non-adaptive byproduct (of some other adaptation or set of adaptations), a by-product that has since taken on new adaptive effect (sometimes called an exaptation) or a cultural technology (a "merely" cultural invention, non-biological).

This discussion can be traced back to an exchange between Charles Darwin and Herbert Spencer. Spencer (1891) argues that music is a by-product of the prosodic/ emotional elements of language, while Darwin (1871) argues that music's origins are in sexual selection (see below) and that language emerged from a musical predecessor. (For a lucid historical background of the debate, beyond the scope of this thesis, see Cross 2007.)

This chapter proceeds as follows. In $\S 1$ I consider the leading accounts of music as an adaptation, and in §2, I consider non-adaptive by-product, exaptation, and technology accounts. And in §3 I critique Lawson's (2014) recent attempt to reconcile "adaptationist" and “technology" perspectives (Killin 2016a). These hypotheses about music's evolutionary status share a common failing: they downplay the importance of co-evolution and niche construction. In my view, this makes the standard set of distinctions (adaptation, by-product, exaptation, technology) rather hopeless for framing the discussion of music's evolution. Understanding music means understanding a patchwork of anatomic, cognitive, 
behavioural, and socio-cultural features, as I detail in chapters 7 and 8 . Nonetheless I suspect that some of the hypotheses discussed are partially successful (that is, they point to an adaptive role for music), and aspects of them will appear in my final two chapters, playing some part in the co-evolutionary model that I develop therein.

Take as an analogy the example of hominin fire behaviours. Fire culture enabled the consumption of cooked meat, softened tubers, and so on, which modified human anatomy through positive feedback: the teeth and gut size of our ancestors reduced, and their brains grew. This amplified hominin fire behaviours, which amplified its effects: the two continued to spiral along in interlocked tandem. This is an example of dynamic bio-cultural coevolution and niche construction. Calling fire control a mere "cultural technology" or "innovation" (e.g., Patel 2010) seems to downplay its dynamic co-evolutionary effects. And it makes little sense to argue, for instance, over whether smaller teeth and guts are adaptations or exaptations to a novel cooking environment. Although neither description is nonsense, the crucial point is that it is not a productive framework in which to couch debate: it does not focus attention on the co-evolutionary dynamics and feedback loops that were key in shaping hominin evolution. In my view, the same goes for music. A re-framing is in order.

So in $\S 4$, I argue the case for a co-evolutionary, niche construction view (Killin 2013, 2016a, 2017). In the remainder of the thesis, chapters 7 and 8 , I then present my positive model of the evolution of music.

\section{Adaptation hypotheses}

Geoffrey Miller (2000) advocates a sexual selection adaptationist hypothesis about music. Notably, this is Charles Darwin's oft-quoted conjecture from The Descent of Man, too:

... primeval man, or rather some early progenitor of man, probably used his voice largely, as does one of the gibbon-apes at the present day, in producing true musical cadences, that is in singing; we may conclude from a widely-spread analogy that this power would have been especially exerted during the courtship of the sexes, serving to express various emotions, as 
love, jealousy, triumph, and serving as a challenge to their rivals. (Darwin 1871, p. 54.)

Darwin's example of gibbons is a good one for arguing for a sexual selection perspective of music. Gibbons (Hylobatidae) are pair-bonding apes; they exhibit low sexual dimorphism, and are highly social and highly territorial. Their complex vocalisations-duets between bonded pairs —are employed in territory defence and pair-bond strengthening. These songs transmit over long distances, often over a kilometre, even in deep forest (Clarke et al. 2006). Gibbons have also been observed to sing as a defence mechanism in response to threat of ground-based predators, such as leopards and tigers, possibly functioning, as per other primates, as a warning call to conspecifics (Tenaza and Tilson 1977) or as a signal of acknowledgement of the predator's presence, a kind of vocal stotting (Zuberbühler et al. 1999).

Bonded gibbon pairs sing before and after mating (see Mithen 2005 for discussion). Female gibbon song (dubbed the "great call") comprises a long succession of sustained tones, often rising in tempo and falling in pitch contour. Singing together appears to strengthen the relationship of the pair-bond as there is a correlation between the relationship strength of a bonded pair and frequency of song. Gibbon couples that demonstrate the closest proximity and the most shared grooming and behavioural synchronisation are the couples that sing together the most. Some experimental evidence regarding the solo vocalisations of gibbons suggests that females sing solo to advertise their territory, and single males sing solo to broadcast their unpaired status. Unlike the long successions of notes in both pair-bonded and solo female song, solo male song comprises a series of short phrases, which usually become more rhythmically complex as the song continues, and is thus easily discriminated from female/pair-bond song. Obviously all this in tandem makes gibbon song a highly likely candidate for a sexual selection adaptationist explanation, and among researchers, sexual selection is considered the primary mechanism for gibbon song evolution (Cowlishaw 1996). Mated pairs sing together, how much they sing together is correlated with the strength of their bond, and unmated males sing a notably different song to advertise themselves. 


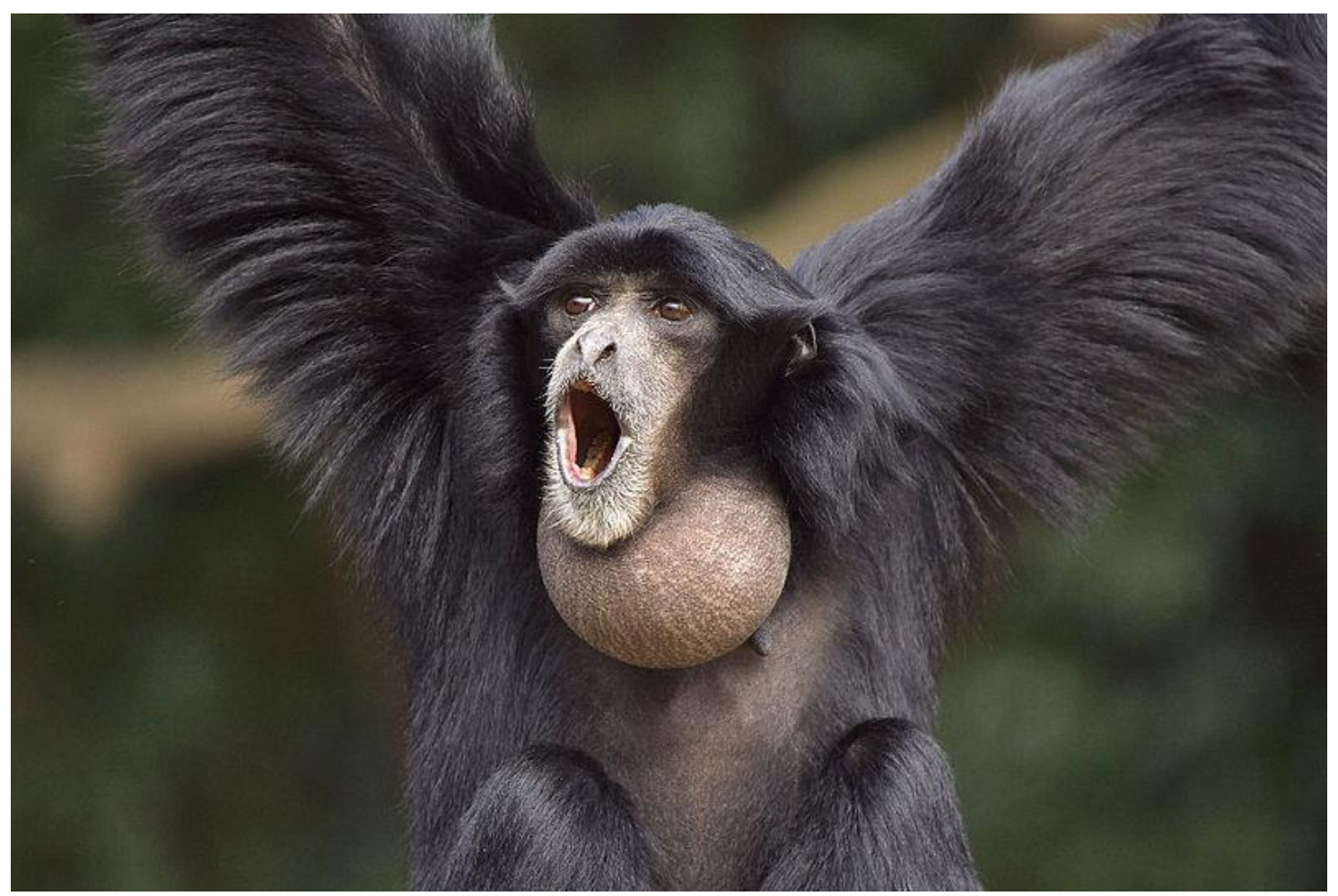

Figure 6.1: Siamang gibbon (Symphalangus syndactylus) ${ }^{61}$

What of hominin music? Is a sexual selection adaptationist account the best explanation? Once something that is deemed valuable or desirable is established, it will play a role in mate choice, to be sure. And music does play this role, in some circumstances, in societies the world over. The Maasai men of Kenya and Tanzania, for example, dance and sing before assembled single women in ritual courtship displays. Other cultures have singing ritualcompetitions, in which male musicians demonstrate their expertise in front of women. And in the West too, music matters in the assessment of partners for many people.

So I am not sceptical of a significant role for music in mate choice in hominin evolution. However, it is unconvincing that sexual selection alone can account for the

\footnotetext{
${ }^{61}$ Photo by Su Neko, freely distributable and adaptable under Creative Commons Attribution 2.0 Generic licence. Source $=$

https://commons.wikimedia.org/wiki/File:Symphalangus_syndactylus,_Chiba_Zoo,_Japan.jpg; Accessed 20/08/2016.
} 
origins of hominin music, even though it almost certainly played a role in its evolution and persistence. The following facts seem at odds with the predictions we would make in order to test the sexual selection adaptationist hypothesis:

(1) Children are capable of demonstrating musical play and even good musical competence well before sexual maturity. This is not the case in standardly accepted examples of sexually selected behaviours.

(2) Cooperative, coordinated, group-based music — rather than individually-focused music - is ubiquitous in the ethnomusicological record.

(3) Lullaby and play-song between mothers/caregivers and infants/children is also ubiquitous in the ethnomusicological record.

(4) Experimental findings suggest there is no correlation between female preference for higher levels of complexity in music (as a proxy for male quality) and occurrent fertility (ovulation), which would be consistent with the sexual selection hypothesis (Charlton et al. 2012).

(5) It has not been established that musicians are any more successful at reproducing than other people (despite the sex-god escapades of a few rock stars and music celebrities), or conversely that non-musicians are less successful reproducers.

(6) Findings from a recent twin study, boasting a huge sample of over 10,000 twins, show that 'genetic correlations between musical aptitude and the measures of mating success were all nonsignificant... findings show that higher musical aptitude or achievement does not lead to increased sexual success (quantitatively)... there was no significant association between musical aptitude and number of children' (Mosing et al. 2015, p. 364).

Again, my point is not that musical skill is ignored in human mate selection. And in the social world of our ancestral past, presumably hominins were forever scrutinising each other for partnership making and unmaking, and music/vocalising presumably played some role in 
that. Rather, my point is that an exclusively sexual selection adaptationist hypothesis is problematic, leaves many things unexplained, and cannot be the whole story.

Turning now to another adaptationist theory, Ellen Dissanayake (1982) argues that art behaviours generally (we may include music here) play a role in making things specialenhancing ritual and, in turn, building a sense of community. For these reasons she thinks that the arts, conceived as a whole, are an adaptation.

In my view, it is not particularly plausible to lump all of the arts together; there is little reason to think that the arts are, or should be conceived as, a unitary natural kind. Work in the cognitive sciences for example supports non-lumping, distinguishing musical cognition from cognition of visual art, and of language (literature, poetry). For example, unlike appreciating visual art, listening to music even when sitting still activates the motor regions of human brains (Mithen 2005). Music's creation and consumption employs an array of neural mechanisms distributed throughout the brain's cartography, mediated on both sides of the brain; language cognition, on the other hand, is predominantly lefthemisphere-though the rhythmical (musical) aspects of poetry and nursery rhyme are mediated on the right (see Orians 2014). And there is no reason to think that the arts emerged in human life at the same time either, or approximately the same time. If that's right, in what sense then are the arts an adaptation?

The arts have certainly not always been grouped together in the modern way. Throughout early Western history, music was categorised alongside mathematics and astronomy—not alongside fiction, painting, dance or sculpture-forming the "Quadrivium" of astronomy, geometry, arithmetic and music, which persisted from the ancient Greeks until the sixteenth century (Deutsch 1984). In ancient Egyptian mythology, although Ptah was the god of various arts and crafts (including sculpture, architecture, metalworking), this did not include music. Music was patronised by the goddess Hathor and dwarf-deity Bes. In China, music was categorised alongside yi: 'ritual, archery, riding, writing, and arithmetic' (Skidelsky 2010, p. 110). Only very recently have music, dance, fiction, poetry, painting, sculpture, and so on, been grouped together; it is the work of eighteenth-century aestheticians that we have to thank for our modern Western art-concept (Shiner 2001; Lopes 2014; Kristeller 1951, 1952). The now-familiar lumping of the arts was eventually 
captured in the Encyclopédie of Diderot and d'Alembert. As this became the dominant paradigm in the West, its outreach expanded and was adopted by some other cultures too, but not all: it is certainly contestable that this concept of art is universal across cultures even today (Monseré 2012). Lumping them in an adaptationist theory appears unmotivated.

Adaptationist theories about the arts as a whole carry extremely unreasonable explanatory burdens regarding universality across the various standard art forms. The upshot: in my view, evolutionary hypotheses ought to target art behaviours separately (music, visual art, storytelling, and so on), not the messy conjunction of them all. Of course, avenues for integrating evolutionary explanations of the various arts may be open. Hodgson and Verpooten (2014) attempt this in their argument that art is a by-product of ritual. That is not necessarily to say that music or any of the other target behaviours are by-products (although that is compatible with their view); rather the idea is that ritual co-opted these behaviours and gave them "art status" in some sense. So their idea that art is a by-product of ritual does not imply that music is likewise a by-product of ritual. Indeed, at least some elements of music might have been required as a precondition for getting ritual off the ground in the first place.

Ian Cross (e.g., 2006, 2007) suggests that music enabled the integration (he calls it the "bridging the gap") of domain-specific neural substrates/modules, giving rise to domain-general cognitive competency. Cross's picture is couched in the framework of Santa Barabara-style Evolutionary Psychology — the massively modular picture of the human mind (see Buss 2005; Barkow et al. 1992)—and, in particular, Mithen's (1996) hypothesis about the breaking down of specialised modules to produce domain-general intelligence. In Cross's view, music provided the key adaptation for engendering human cumulative culture, given music's so-called "floating intentionality". By this, Cross means that since a musical expression could be intended as "about" diverse things in myriad contexts, music's use enabled hominins to break out of their domain-specific psychology, in which particular mental modules are dedicated to (or "about") a unitary facet, and develop domain-general competency (in which the various modules interact). This model of the mind, however, has seen strong criticism in recent years and I am among the sceptics. Opponents argue that such a model unduly black-boxes the cultural from the biological (Currie and Killin 2016), is incompatible with the profound plasticity of the human mind (Sterelny 2012b), and 
incompatible with the best theories of the nature of evolution (for review, see Downes 2010). Moreover, although musical expressions can be intended as "about" a great variety of things $s^{62}$, the idea that music is then an adaptation-its proper function supposedly 'underwriting social flexibility and in facilitating intellectual flexibility' (Cross 2006, p. 123)— however, is bewildering (and the hypothesis very unclear: what is the proposed mechanism? How was musical cognition supposed to tunnel between domain-specific modules?). As Stephen Davies notes: 'Who would have thought that the absence of specific meaning in music would have such far-reaching consequences!' (Davies 2012b, p. 179). And presumably fire control, stone tools, and many other important aspects of ancient hominin life, also do not have specific meanings. Couldn't these things have, in principle, facilitated the domain-general breakthrough instead of music, on Cross's picture? (That is, if I were to accept for the moment the Santa Barbara-style modular model of mind, which I do not.) So it is not clear from the outset that the "floating intentionality" of a musical expression is really distinctive in some relevantly salient way. For further critical discussion of Cross's account, see Davies (2012b, pp. 179-182). Unlike some of the other adaptationist hypotheses (sexual selection—see above-parent/infant affect, and cooperation/coordination-see below), which point to plausible adaptive roles, in my view Cross's hypothesis is not part of the right story.

A fourth adaptationist hypothesis for music is associated with (the later) Dissanayake (2000a, 2000b, 2008, 2009) as well as Sandra Trehub (e.g., 2001, 2003) and various collaborators. This band of researchers emphasise developmental evidence suggestive of a cross-cultural infant sensitivity to elements of music. Mothers/carers in all known cultures vocalise to their infants with a music-like tone (heightened contours, emphasised vowels, repeated rhythms, and dramatic pauses), and these 'exaggerated signals are processed as "good" and "affiliative" in the infant's brain, where they are translated into emotions of delight and pleasure' (Orians 2014, p. 129). Lullabies and play-songs are an extremely widespread cross-cultural phenomenon, sharing various similarities (Trehub 2000; Unyk et al. 1992) despite some variability in musical style.

\footnotetext{
${ }^{62}$ However, even this is not obvious, in the referential sense of intentional. Music is purposeful, and can be expressive and thus representational (which is a sense of "intentional") but music is not referential. Cross's concept of "floating intentionality" then might be confusing two concepts of the intentional.
} 
The adaptationist hypothesis here implies that music enhanced survival fitness through reinforcing affective bonds between infant and mother/carer, and providing mothers/carers with a means to modify attention, arousal and affect, as well as induce sleep in (rather helpless) infants, a good survival trick in any environment. I agree with the basic line that these researchers are running: I think that infant-directed vocalising (Dissanayake 2000b envisions vocal-facial-kinesic affective sequences) would have played an adaptive role in hominin evolution and it will feature in my co-evolutionary model (chapters 7-8). But as with the sexual selection adaptationist hypothesis, I do not think that the "motherese" adaptationist hypothesis can be the whole story. Again, I think music is a co-evolving patchwork of features. The adaptationist rendering of music posits a unitary explanation for a myriad phenomenon (Tomlinson 2015). This hypothesis leaves too much unexplained (for instance, music's role in mate selection, music's role in coordination/cooperation).

Fifth, there are a cluster of hypotheses that emphasise some aspect of coordination/cooperation. Brown (2000) suggests that music reinforced "groupishness", appealing to the notion of multi-level selection. Hagen and Bryant (2003) suggest that music functioned as a coalitional signalling system. The later Cross (2012a, c), suggests that music functioned as a medium for the management of unclear or ambiguous social situations. Dunbar (1996) argues that "vocal grooming"- -affective vocalisations from individuals to members of the group-functioned as social glue. As above, I think that cooperative, coordinated music would have played an adaptive role in hominin evolution, as I detail in the following chapter. But, as above, it is not the entire story.

Finally, hybrid adaptationist accounts have been proposed, that combine elements of several of these unitary adaptationist hypotheses (Mithen 2005: vocal grooming, mother-infant displays, sexual selection). Mithen posits a musilanguage, a vocal forerunner of both language and music. I will critique the idea of a musilanguage in §3. But also note that appealing to multiple proper functions of music in an adaptationist account has been criticised methodologically (Davies 2012b; Tomlinson 2015) and in any case is not required for an evolutionary model of music to make use of several adaptive roles for music. Indeed, I take the co-evolutionary model that I develop in the next two chapters as a foil to a hybrid adaptationist strategy, though one that will share many complementary aspects. 


\section{Non-adaptive by-product, exaptation, and cultural technology hypotheses}

That music is a by-product is perhaps the majority view (see e.g., Pinker 1997; Sperber 1996; Barrow 2005; Panksepp 2009). However, as Davies (2012b) points out, theorists that advocate by-product accounts of some trait do not thereby have an easier explanatory duty than those who defend adaptationist accounts. A reasonable by-product hypothesis should, for instance, provide a cogent account of the adaptation or suite of adaptations that the trait is said to be a by-product of.

Steven Pinker (1997) argues that although music, in his view, is not a biologically or cognitively important phenomenon, it nevertheless stimulates auditory, spatio-temporal, and motor functions in human brains which accounts for our fascination with music, its scope, ubiquity, and persistence, and so on:

As far as biological cause and effect are concerned, music is useless... Compared with language, vision, social reasoning, and physical know-how, music could vanish from our species and the rest of our lifestyle would be virtually unchanged. Music appears to be a pure pleasure technology, a cocktail of recreational drugs that we ingest through the ear to stimulate a mass of pleasure circuits at once. (Pinker 1997, p. 528.)

I suspect that music is auditory cheesecake, an exquisite confection crafted to tickle the sensitive spots of at least six of our mental faculties. (Pinker 1997, p. 534.)

Pinker hypothesises that the six mental faculties that interface with music are language, auditory scene analysis, emotional calls, habitat selection, motor control, and 'something else... that explains how the whole is more than the sum of the parts' (Pinker 1997, p. 538). On this view, music does not have a deep connection with human biology, but 
merely generates pleasure rewards, the underlying mechanisms of which were originally selected for in other contexts. That is, music is a non-adaptive by-product. ${ }^{63}$

Similarly, for Dan Sperber, 'music... is parasitic on a cognitive module the proper domain of which pre-existed music and had nothing to do with it' (Sperber 1996, p. 142). For Sperber, that specialised module was built for detecting human communicative sounds and rewarding detection. Sperber's idea is that once this module was in place, human music (and Sperber adds birdsong) artificially triggered the reward mechanism associated with this domain-specific module. However, both Pinker's and Sperber's hypotheses leave unexplained the origins and emergence of music. (And see above for my departure from the Santa Barbara-style modular theory of the mind, the framework invoked in both cases.)

However, even if Pinker, Sperber, and others are right (generally speaking) that music was initially a by-product of some kind or other, there would have been co-evolutionary consequences once music was a part of the hominin social environment. It would not have remained selectively neutral. The redness of mammalian blood, for example, is a by-product of blood's biochemistry and function (internal, iron-based oxygen transport). So blood's redness is merely an indirect result of selection (it's not that selection conferred adaptive fitness on red-blooded mammalian ancestors versus their green- or blue-blooded competitors). So the colour of blood is selectively neutral. (And for obvious reasons, the colour of blood cannot be co-opted as, for example, a social or sexual signal.) I will argue that this is not the case with music. If music were initially a by-product (and remember, it is far from clear that it is), it would have taken on adaptive effect.

Traits that take on some new adaptive role-that is, "features that now enhance fitness but were not built by natural selection for their current role" (Gould and Vrba 1982, p. 4) are known as exaptations. As Gould and Vrba make the distinction, while adaptations have proper functions, exaptations have adaptive effects. The foot of the ancient (150 Mya) Archaeopteryx was not an adaptation for settling in trees, yet tree-nesting gave the ancestral bird certain advantages, so its foot design came to exert an additional adaptive effect-tree-nesting-after features of the foot co-evolved within the bird's novel tree-

\footnotetext{
${ }^{63}$ For objections to Pinker's "cheesecake" and "recreational drug" analogies, see e.g., Carroll (1998); Dutton (2009); Davies (2012b). I provide some reasons for thinking that music is not biologically/cognitively neutral (let alone detrimental) in discussion that follows.
} 
nesting niche. Similarly, feathers evolved initially for thermoregulation, but have since been exapted for the purpose of flight (and thus many structural features of feathers are adaptations for flight). Some researchers suggest that the enlarged, masculinised genitalia of spotted hyena females are a by-product of selection for high testosterone titers enabling female dominance and larger body size (Gould 1997; Gould and Vrba 1982) rather than a direct result of selection (Kruuk 1972). However, if the enlarged genitalia of hyena females have been co-opted by selection for an adaptive role, as they plausibly have in hyena meeting ceremonies ${ }^{64}$, then they are perhaps better categorised as exaptations ("offshoots" for that adaptive effect) than mere (non-adaptive) by-products, such as blood's redness.

Although Davies does not fully endorse it, and avoids the use of "exaptation", the above line of reasoning appears to amount to his preferred view of music in The Artful Species - that even if music is not an adaptation, it would have come to exert adaptive effects. The idea is captured by his slogan "form becomes norm" (Davies 2012b, p. 144).

If art behaviours came to us as ancillary evolutionary by-products, they would not remain merely incidental. Their occurrence in the usual manner would become normative because they provide honest, because costly, signals of fitness. As a result, not only the absence of art behaviours but also the degrees to which they are represented can be informationally significant in assessing someone's fitness. (Davies 2012b, p. 145.)

Before proceeding I should flag that I am somewhat sympathetic to Davies' move here, and his dynamic conception of biological and cultural evolution. However, I will go on to develop the core idea outside of the adaptation/by-product framework for discussing

\footnotetext{
${ }^{64}$ Hyenas live in social groups but also spend much time alone on solitary scavenging expeditions. When a wandering hyena rejoins its clan, it presents its genitals for olfactory inspection from a conspecific to establish that it indeed belongs in that clan (or not, as the case may be). See Kruuk (1972).
} 
music's evolution (Killin 2013; cf. Davies 2012b, though supported in principle by Davies 2017).65

A fourth view is that music is a cultural technology/innovation. A prominent technology theorist is Aniruddh Patel, according to whom music is a 'transformative technology of the mind' (Patel 2010, p. 91). According to Patel, neuroscience research suggests that music can have a profound effect on people's brain structures over the course of their lives: it shapes the neural structures of individuals and has genuine effects on their development, through for example the effects of neuroendocrine hormone-release and the brain's great plasticity. So music is not a non-adaptive by-product on Patel's view. Nonetheless, Patel thinks that music has not been targeted by selection so it is not an adaptation nor an exaptation, either. (This is in contrast to Davies's view that once an activity as central and ubiquitous as music emerged it would not stay selectively unimportant.) Music can be used to some effect in therapy—rhythmic stimulus exercises can aid the rehabilitation and gait control of stroke patients (Thaut et al. 1997), hospitals have used music in order to calm patients and lessen their desire for self-administered pain medication during locally-anaesthetised operations (Ayoub et al. 2005), and lullabies have a beneficial effect on the feeding and development of prematurely-born infants (Standley 2000, 2003, 2012), for example. Conversely, music has been used for torture in prisons and military bases (Sharrock 2010; Meehan 1997). Music can calm and soothe as well as excite; it can even incite violence. There is evidence that learning music increases general intelligence (Schellenberg 2004, 2005, 2006) as well as increases competency in mathematics and the ability to detect a speaker's emotions (e.g., Gardiner 2008; Thompson et al. 2004; see Schellenberg 2016 for review). Yet, if music can shape our ontogeny so, very plausibly it can shape phylogeny too. Patel might be right not to call music an adaptation, but he is too quick to assume that it would not be picked up on by selection.

So far I have reviewed accounts of music as adaptation, by-product, exaptation, and technology. Objections to any given account aside, the crucial failing of these hypotheses

\footnotetext{
${ }^{65}$ Also, on his The Artful Species blog, Davies tells us he has 'come to think these categories are not for talking about human evolution. We don't adapt to our environment, we create the environment that suits us... As a result, there are feedback loops in terms of which culture affects biology and biology affects culture. So the notion of adaptation does not apply to us in the way it does to most other species.' See https://artfulspecies.wordpress.com. Accessed 17/08/2016.
} 
(with the exception of the updated view of Davies) is that they acquiesce in a framework for conceptualising evolution that does not take seriously the implications of gene-culture co-evolution and niche construction, and does not focus attention on the key interaction of biology and culture. I will argue my case for reframing the discussion in these terms in $\S 4$. First, however, I will consider and reject Lawson's (2014) recent attempt to demonstrate how the adaptation and technology accounts of music might be reconcilable.

\section{Lawson's "reconciliatory" evolutionary model}

In her Jaap Kunst Prize-winning article, Lawson (2014) draws attention to the rich connection between music and language often downplayed by some theorists. She provides an intriguing case study-Chinese shuochang, a contemporary sung-spoken vocal performance tradition-that illustrates just how profound that connection can be, especially in conjunction with visual orthographies that feed back into the aural/oral aspects of the phenomenon. This research is fascinating for a number of reasons. It can contribute to a bigger picture showing how music and its notation co-evolve, and it highlights potential cognitive consequences of learning from different notational systems, given the different aspects of music that distinct notational systems privilege. That said, I am sceptical of Lawson's use of the case of shuochang in evolutionary theorising about music's purported status as an adaptation. Thus I critique accordingly; not to criticise her fine ethnomusicological research but her evolutionary model as she presents it in her article (following my critique in Killin 2016a).

Lawson aims to contrast and reconcile two hypotheses about music's evolutionary status, that of adaptation and technology. She suggests that the research presented in Malloch and Trevarthen (2010) provides compelling evidence for an ancient precursor to music and language, "musilanguage” (see also Mithen 2005; Wray 2002, 2008). Lawson focuses on communicative musicality — by which she means the music-like communicative interaction between mothers and infants (see Lawson 2014, pp. 4-5), which she thinks is an adaptation. Lawson also suggests that musical practices are technologised - that is, influenced through the use of visual orthography—and that music, in general, fits Patel's model of transformative technology, discussed earlier. In sum, then, Lawson's view is: 
... most music, especially that created using a written orthography, is an example of a transformative technology... However I would also argue that... musilanguage, as the precursor to music and language, is a biologically rooted human capacity, evidenced by its continued presence in the well-documented interaction between human mothers and their prelinguistic infants. (Lawson 2014, p. 8.)

Lawson's evolutionary model looks something like this: (1) at some point in our lineage's past (she does not say when), our hominin ancestors developed a musilanguagean adaptation —of which a function was "communicative musicality", that is, mother-infant vocal interaction; (2) remnants of this musilanguage in contemporary humans include our musical practices, our fully-fledged languages, and the way in which we interact vocally with neonates-our infant-directed vocalisations.

I agree with Lawson that mother-infant vocalisations would have played an adaptive role in human evolution. And presumably they still do. However, the musilanguage model that she proposes is not well supported, and I do not find her argument particularly persuasive. In an attempt to lend support to her musilanguage hypothesis, Lawson examines the traditional practice of shuochang, describing it as a 'modern Chinese form of musilanguage' (Lawson 2014, p. 8). But shuochang is not a musilanguage-it is not an antecedent of emergent, novel linguistic and musical systems. Shuochang is a vocal artistic practice that combines elements of existing, established sung/spoken norms and conventions; it encodes propositional/semantic/syntactic content, rather than merely emotional/affective and basic proto-linguistic content typically assumed of musilanguage. And presumably it is practiced by fully competent speakers of a modern language. So shuochang might well be a model for a music/language hybrid that, say, demonstrates 'the tenacity of lexical tone and its musical play in the language-music relationships of a modern genre' (Lawson 2014, p. 13), but it is not clear how its analysis and description could speak to the evolutionary status of a hypothesised precursor to both music and language, since it utilises those forms. (Indeed, similar reasoning explains why theorists must take great care when using chimpanzees as models for ancient hominins.) That is not to say that 
shuochang's research has no upshots for scientific debate. The different ways in which notation is used in shuochang among its practitioners may well give rise to different cognitive consequences, reflecting how 'aural and visual learning processes influence music' (Lawson 2014, p. 24), but this does not weigh in on the music-as-adaptation debate.

Indeed, focussing on visual orthographies seems misplaced in adaptationist theorising about music. Notation systems are very recent in evolutionary time, yet music is very ancient. The oldest known musical notation systems (on cuneiform tablets) are but 3,400 years old. The tradition of notated Western music harks back little more than one thousand years (Treitler 1982, 1992). Moreover, although Western musical notation is now very far-reaching, this is an effect of only the last 150 years or so. Thus, non-notated music - from the whole-group musical activities of hunter-gatherer bands, to aurally/orally transmitted folk traditions-represents the proper test cases for exploring the evolutionary origins of music, not notated music.

There are several other critical points to make here. First, Lawson seems to me to be in danger of conflating "adaptation" and "adaptive trait". Adaptations need not be adaptive traits and adaptive traits need not be adaptations; the term "adaptation" is backwards-looking, "adaptive trait" forwards-looking. Our "sweet tooth" disposition for sugary and fatty foods is an adaptation: it was beneficial to our Pleistocene ancestors to seek out fruits and animal fat. Since then our environment has changed and sweet and fatty foods are ubiquitous, so plausibly that disposition is no longer adaptive-perhaps even maladaptive-given the rise of obesity and diabetes in developed countries. Similarly, by-products need not be non-adaptive. Literacy may be adaptive, but it is presumably the by-product of cognitive adaptations for language, pattern recognition and social learning. The cognitive mechanisms that enable literacy probably have not been much altered by selection for literacy, but if literacy increases an individual's fitness, then it is adaptive. In her characterisation of the adaptation perspective, Lawson describes the view as one that postulates that communicative musicality is adaptive, thus not distinguishing a truly 
adaptationist account from an exaptation account. So Lawson's adaptationist perspective is elusive. ${ }^{66}$

At the same time, Lawson describes Patel's view as one that 'does not believe music is an adaptation, but considers it biologically significant as a transformative technology' (Lawson 2014, p. 4). Obviously, one could deny that music is an adaptation (or that it is adaptive), yet assert that communicative musicality is, assuming roughly that "music" refers to traits of individual phenotypes which enable particular group or individual sound-based artsy behaviours, and that "communicative musicality" refers to mother-infant communicative vocal interaction. So, there is no immediate dispute emerging from the way Lawson sets up the dialectic between the adaptation and technology perspectives: one is said to be about communicative musicality, the other about music; their explananda are different. So her goal of demonstrating 'how these seemingly conflicting viewpoints... might be reconciled' (Lawson 2014, p. 4) may well be achieved at the outset, trivially stipulated.

Besides, something very important remains missing from Lawson's picture. As Robin Dunbar points out:

... humans seem to engage in music as a social activity... Humans use music as a mechanism for community bonding... In modern societies, we may often sit listening politely to music in concert halls, but in traditional societies music-making, song and dance are almost indistinguishable and play a crucially important role. (Dunbar 2014a, p. 19.)

Music is often made collaboratively and cooperatively, in groups, in a grouporiented way that is quite unlike mother-infant vocalisation. Although music may not always be group-focused, this is a crucial aspect of typical music-making across cultures and historic eras. Lawson's focus on mother-infant vocalisations does not provide an adequate evolutionary explanation of the group-based, collaborative nature of music. So although

\footnotetext{
${ }^{66}$ Admittedly, Lawson briefly distinguishes the views that she discusses from the "mere by-product" account by citing Pinker (1997), discussed above in $\S 2$.
} 
Lawson weighs in with her view that communicative musicality is adaptive, in terms of motivating an adapationist account of music, de facto Lawson contributes little.

It is also worth bearing in mind that the research in Malloch and Trevarthen (2010) that Lawson cites supports a number of other hypotheses too. It does not entail Lawson's model. For instance, ancient vocal musicality may have provided the medium for spoken language, once (proto-)language evolved from gestural/manual, rather than vocal, means (see chapter 7 of this thesis). Proponents of this view argue that language evolved not from some common ancestor with music/song (i.e., a musilanguage), but from the top-down (intentional) aspects of great ape communication. Most of these are gestural, not vocal (see e.g., Hobaiter and Byrne 2014; Pika and Mitani 2006). There are a number of reasons to prefer this hypothesis: (i) there is evidence that human gestural and vocal language employ similar neural systems (see e.g., Newman et al. 2002; Kimura 1993); (ii) these neural systems are largely distinct from areas of the brain associated with emotion ${ }^{67}$ (which are employed in primate vocal communication such as the famous reactive/automatic vervet monkey alarm calls: see Seyfarth and Cheney 1990); (iii) that gestural communication is ontogenetically prior to vocal communication in human infants; and (iv) that gesture remains a wide-spread adjunct, intentionally as well as unintentionally, in many contexts of human vocal communication. All this undermines Lawson's conjecture, 'that maternalinfant interaction not only represents the foundation for music but for all human communication' (Lawson 2014, p. 5). Of this I am very sceptical. In my view, the evidence suggests that music/communicative musicality and language originate largely from separate domains—vocal and gestural, respectively.

Moreover, Lawson suggests that music notation is a transformative technology, in the sense of Patel. Her particular example is Western classical art music and the surprising turns it has taken since the development of its notation system: 'The transition from oral/aural performance to performance that utilises notated scores began a process whereby musical notation became increasingly detailed in terms of visual data presented and, therefore, the learning and transmitting of music was fundamentally changed'

\footnotetext{
${ }^{67}$ Emotions are largely a right-brain phenomena; language is largely left-brained. So most humans find it surprisingly difficult to critically reflect on their emotional states and to describe those emotional states in words (Gamble et al. 2014, see p. 49). Music, on the other hand, involving a broad cartography of the brain, is easily expressive of emotions.
} 
(Lawson 2014, p. 10). The notation of Western art music presumably had important upshots and fed back into its ever-evolving career, as well as the learning, appreciation, and perception of music.

However, Lawson's further claim, that 'humans seem to be predisposed to technologising [= influence by writing down] musilanguage' (Lawson 2014, p. 24) warrants much scepticism. I reiterate that the majority of the world's music was not written down until very recently and notating music has not always revolutionised musical practice. Take, for example, central Javanese gamelan music, and its conventional cipher notation developed around the turn of the nineteenth/twentieth centuries. As is well known in the ethnomusicological literature, the notation has come to be used as a mnemonic and archival device and by gamelan musicians in rehearsals, but is rarely used in performance or serious teaching. It is doubtful that the notation has had the transformative effect on gamelan music that notation had on Western art music, and the gamelan musical tradition remains to be considered one that is primarily aural/oral, despite having a conventional visual orthography. Moreover, notation appears to have played a minor role in the contemporary and experimental music of Javanese composers, and its use at all remains a topic of debate among expert gamelan musicians and composers (see e.g., Sadra and Diamond 1991).

The same is plausible of the development of blues music, for instance. From calland-response work songs to modern forms, the blues tradition and key musicians therein seem to have been little influenced by notation, despite the widespread publishing of ragtime and blues songs by American publishing houses from the $1890 \mathrm{~s}$. In my view, it is too far a stretch to label the "technologising" phenomenon as a human predisposition, as Lawson does.

Finally, Lawson suggests that 'modern music could well be a technology' (Lawson 2014, p. 15), leaving the conjecture rather open-ended. Plausibly, modern music-conceived as the acoustic product of collective, social practices-might be the target of technology theories (just as musical notation is also a product of those collective, social practices), but it is not obvious that Lawson's argument captures music conceived as a trait or series of traits of individual phenotypes underwriting those collective, social practices. And this 
distinction belies a deeper issue: perhaps splitting music's evolution in this way is misguided, if the biological and cultural aspects of music have co-evolved. As I explain in the next section, this would undermine the standard set of distinctions-adaptation, byproduct, exaptation, technology-in evolutionary theorising about complex behaviours. ${ }^{68}$

\section{Co-evolution and niche construction}

Recall the example of hominin fire behaviour from this chapter's beginning. Sustained fire control appears around 790 Kya (Goren-Inbar et al. 2004), becoming more continuous and archaeologically visible from around $400 \mathrm{Kya}$. Some theorists put it as far back as the Habiline/Erectine transition, a million or so years earlier (Wrangham 2009; for review, see Attwell et al. 2015). In any case, it is uncontroversial that fire control is an ancient and important aspect of hominin life.

Fire enabled the cooking of meat and tubers, making available the increased energy needed for encephalisation. It bought our ancestors more time for other pursuits especially in the social realm, both by extending their day (increased light from fire) and softening their foods (compare with chimpanzees, who spend hours every day chewing-cooking softens foods and breaks down proteins, making foods easier to consume and digest).

Fire control fed back into hominin anatomy (Wrangham and Conklin-Brittain 2003), reducing the teeth and gut size of our ancestors, allowing reallocation of developmental energy to encephalisation, and so on. The heat from cooking killed off harmful pathogens present in food items, easing digestion and relaxing genetic selection for pathogen resistance. These are examples of gene-culture co-evolution: fire culture and hominin biology evolved dynamically. (For example, mutation of gene $\mathrm{MYH} 16$ is thought to be responsible for the weakening of our ancestor's jaw muscles; Wrangham (2009) connects this with early cooking activities.) So, contra Patel, it is not actually helpful to conceive of fire as a technology, artificially splitting the cultural from the biological. Doing so overlooks the intertwined, dynamic co-evolutionary forces at work.

\footnotetext{
${ }^{68}$ I do not deny that it is still useful to classify the heart and eye as adaptations, the redness of blood as a by-product, and so on. Gene-culture co-evolution and niche construction do not add anything to the explanation of the evolution of those traits.
} 
Other examples of gene-culture co-evolution in the hominin lineage include the coevolution of the hominin hand and tool use/production (Marzke 2013), and the co-evolution of cattle farming and increased tolerance of human adults to lactose (Beja-Pereira et al. 2003). Dynamic interaction between biology and culture can thus drive evolution into surprising areas unpredictable by the lights of the standard conception of traditional evolutionary explanation and the adaptation/by-product framework. That conception posits a reasonably stable environment to which certain phenotypes arise in response, assuming a straightforward causal picture (a one-way environment to phenotype causal arrow). In co-evolutionary scenarios involving hominins, the hominin social environment is not a mere "enforcer" of selection, but an output that is altered along with the phenotypes, through feedback, on which selection operates. In my view, music requires a co-evolutionary treatment.

Brown et al. (2013) show that there are significant correlations between genes (specifically, mitochondrial DNA haplotypes) and music (group-performed vocal pieces) in nine geographically distinct indigenous populations of Taiwan. Their research indicates that musical diversity and genetic diversity are significantly correlated, with musical overlap between that of nearby regions reflecting shared ancestry (genes) and not just inter-group cultural diffusion. From the results of their study, these researchers conclude: 'The correlations we observed between musical and genetic diversity support the contention that music and genes may have been co-evolving for a significant time period and that music might possess the capacity to track population changes' (Brown et al. 2013, p. 5). Of course, this is evidence of indirect co-evolution, not evidence for co-evolving "genes for music".

Savage et al. (2015) report similarly striking correlations between Ainu music and mitochondrial DNA types. Twin studies add to the argument for co-evolution (e.g., Hambrick and Tucker-Drob 2015), as do several well-known features: music is widely culturally variable, although ubiquitous, present in all known human societies, and follows a (seemingly) fairly structured developmental sequence, despite the very real influence of culture-specific musical exposure on the development of musical cognitive capacities (see e.g., Hannon and Trainor 2007; Schellenberg and Trainor 1996; Schellenberg and Trehub 
1996a, b, 1999; Soley and Hannon 2010; Stalinski and Schellenberg 2012; Trainor 2005; Trainor and Corrigall 2010).

So as Richard Menary has recommended in the wider context of evolutionary aesthetics, 'if we think of the evolution of art as a co-evolution of biological capacities with culture, we may be able to provide a more accurate account of how our aesthetic traits evolved' (Menary 2014, p. 475). In chapters 7 and 8 I provide my co-evolutionary model of this kind for the case of music.

First, however, here I consider music in the context of niche construction, generally. One way that ethnomusicologists can fruitfully contribute to evolution of music research is by taking seriously such a perspective (more so than under the adaptationist framework). Living organisms alter their environments through their activities, shaping the living conditions of themselves, their offspring, and other affected organisms: beavers, for example, drastically alter their environments, and the local ecosystem at large, by building dams. (For an introduction to niche construction theory, see Odling-Smee et al. 2003.) Through feedback loops, changing living conditions affect the development, actions, and selection of organisms down the evolutionary trail. This is especially true when the environmental effects are persistent or cumulative. And human populations in particular flourish in large part because humans themselves construct the environments that they experience (Kendal et al. 2011). Turning to ethnomusicology can reveal some of the ways in which music plays a significant role in processes of niche construction (potentially leading to fitness differences), and music's roles in the social group and wider context.

The anthropologist Jean L. Briggs has carried out fieldwork on Canadian Inuit forager life since the 1960s. Her research includes descriptions of the twentieth century transition undertaken by Inuit groups from small-camp nomadic forager lifeways to largegroup communities with established settlements. Such a transition involves a revolution in social niche, and Briggs stresses its consequences well:

To put the situation in the simplest—perhaps oversimple-terms, nomadic Inuit, moving into a settlement, exchange physical hardship and (relative) social ease for physical ease and social hardship. On the physical side, no matter how inadequate the diet in the settlement is felt to be, there is no 
longer need to fear starvation; no matter how ill people get, there are nurses and doctors to tend them; and if one has no means of earning money, welfare will, no matter how inadequately, provide. But on the social side, a great many unfamiliar and difficult situations are now encountered. Uncertainties with which Inuit had established ways of dealing have been replaced by uncertainties that they do not know how to manage. (Briggs 2000, p. 110.)

Briggs describes how music took on a social management role in the development of the new large-group communities. Hostility amongst Inuit adults was ritualised, and publically dealt with through song duels. If 'the feelings of the antagonists ran too high to allow them to keep silent' (Briggs 2000, p. 111), the rivals would exchange scornful, sarcastic songs before their amused onlookers:

In short, the duel embedded conflict in an artistic form, isolated it within a ritualised context, concealed it behind irony and an ambiguity of genre, and at the same time publicised it by focusing the attention of the entire community on it. (Briggs 2000, p. 112.)

Insofar as this social management strategy was successful in deterring actual conflict and controlling adult hostility, music/vocalisation had been put to use for a sociopsychological grooming effect also recognised in other primates. Geladas, for example, who live in very large groups (up to 200-300 individuals) organised into long-term harems (each comprising one adult male, up to ten females and dependent offspring) are an excellent comparative example (Gustison et al. 2012; Richman 2000). A harem leader male will use vocal calls to pacify females engaged in hostile exchanges, and much gelada vocalisation can be interpreted as playing a socio-psychological grooming role (Richman 1987, 2000). Obviously, geladas are not a model for Inuit people; my point is that using vocalisation/song for maintaining social cohesion is by no means unique to humans (although it may be unique to humans among the great apes). 
Inuit children who grow up in a society that values song duels as means to working out adult disputes know what is expected of them once they reach adulthood, if they hold grudges or form antagonistic relationships with peers. The song duel tradition is thus reinforced and remains in place for subsequent generations at least in principle, barring some other cultural revolution or strong new influence. An individual who is expected to participate in a song dual but refuses could very well reveal themselves as a social defector; the costs of refusal could be much greater than the potential costs of undesired participation (public embarrassment, perhaps).

So the use of music in conflict management, especially in contexts of social transition, is an excellent area of research wherein ethnomusicologists can shed light on upshots for both niche construction theory and theories of music's adaptive effects. After all, the actions and behaviours of the current generation alter the cultural/informational landscape — the world of norms, conventions, innovations and shared knowledge-for future generations:

[Humans develop through] interaction with family, friends, collaborators, strangers, and domesticated animals, and an environment loaded with human artefacts... All of this social and material infrastructure scaffolds the development of human cognitive phenotypes in every culture today and has for many thousands of years. (Trestman 2015, p. 87.)

Children that grow up in a social environment in which much information is expressed through traditional music, for instance, develop in a context that is scaffolded by music and its role in informational and cultural transmission and social learning. The traditional lifeways of Australian Aborigines is one such example, maintaining highly symbolic, lyrically-dominated song traditions tied closely to geography, land ownership, story and ritual, and which maps their vast, barren, homogeneous desert environments.

I will have more to say on music in traditional/hunter-gatherer societies in chapter 8. The crucial point for now is that a niche construction perspectove provides a more productive framework for contributing to evolution of music research than the 
adaptationist debate. This is not to endorse crude theorising by ethnographic analogy: to be sure, researchers must be wary of overzealous inference about our ancestors from observing extant peoples. Yet turning to the ethnographic record allows researchers to explore some ways in which music is involved within processes of niche construction, and music's roles within the social group and wider context. It allows us better to understand ways in which foragers living under similar conditions might be musical, helping to constrain our evolutionary hypotheses. It helps to explain how music can be co-opted in social transitions, and it reveals similarities and differences across social groups that can be systematically studied, providing data for evolutionary models.

This foregrounds a methodological concern within music academia and I shall pause here to advocate a largely untapped research programme for ethnomusicologists and other music researchers. Although comparative ethnographic studies illuminate the diversity of musical traditions around the world, as well as any statistical universals (inter- and intraculturally), most professional ethnomusicologists today primarily focus on music's analysis through poststructuralist, postmodernist, phenomenological, political/economic (for instance, Marxist) agendas or perspectives, among others (Stone 2008). Some researchers have called for the return of (a somewhat upgraded) comparative ethnomusicology (see e.g., Savage and Brown 2013) and I am sympathetic (Killin 2014, 2016a).

Such a research agenda (in tandem with the others) could complement and integrate much ethnomusicological research and feed into scientific debate. Research projects in comparative musicology have the potential to reveal, in a quantifiable manner, musical diversity as well as similarity (including any statistical universals), as well as provide data to fuel phylogenetic modelling of musical traditions-as recent linguistic research has done, underwriting potentially information-rich models of language evolution (see e.g., Gray et al. 2010, 2011) and evolution of writing systems (for discussion, see Lock and Gers 2012). Just as different biological species have different traits (for example, chimpanzees have longer arms than legs, while the opposite is true of humans), the different traits of musical systems can be systematically studied and catalogued, and then analysed using phylogenetic algorithms to generate models. Russell Gray and colleagues' (2011) phylogenetic models of Austronesian languages enable researchers to test hypotheses about the dates and patterns of migration of Oceanic populations, for example. 
And Rzeszutek et al. (2012) construct phylogenetic models mapping the diversity of songs in Austronesian languages, allowing researchers to begin to test hypotheses about the cultural diversity within human groups by quantifying the hierarchical structure of that diversity, using songs as the unit of analysis.

Phylogenetic modelling of aspects of the world's musical traditions is an exciting, although largely underexplored, prospect for elucidating the nature of the evolution of music. Well-confirmed phylogenetic models can allow theorists to test hypotheses about the evolution of music and changes in music's socio-cultural roles, analyse musical change over time, and scientifically test the conclusions of other research methods. When phylogenetic systematic analyses overlap with hypotheses generated from other lines of evidence (for instance, the archaeological record), theorists are in a better position to evaluate competing evolutionary hypotheses, appealing to the convergence of independent data points. And these phylogenetic approaches are also independent of the adaptation/by-product framing of previous discussion about the evolution of music.

\section{Towards a co-evolutionary, socio-cognitive niche model of music}

In this chapter I have surveyed hypotheses of the evolutionary status of music and I have called into question the usefulness of the adaptationist framework (and the standard distinctions therein) in which this discussion is largely contained. That framework acquiesces in a one-way causal conception of evolution (that is, environment begetting phenotype) that ignores the dynamic consequences of co-evolution and niche construction, promoting an unjustified separation of the cultural from the biological.

An alternative framework for hypotheses of music's evolution emphasises niche construction and promotes the development and critique of phylogenetically-plausible evolutionary scenarios for music's emergence-historical narratives that take into account co-evolutionary processes and multiple lines of evidence, connected up to a bigger picture of hominin evolution. In this research programme, theorists construct and evaluate lineage explanations (Calcott 2009) of music -narratives that track between series of phenotypes and hook up to motivated inferences from the evidence. In what follows, I motivate and develop my argument for my model of this kind. 


\section{Chapter 7: Evolutionary foundations of musicality}

As outlined in chapter 6, recent discussion about music and evolution is largely couched in the adaptation/by-product framework. And as I argued in that chapter, seeking an alternative approach is well-motivated. One alternative framework for the discussion promotes the development and evaluation of phylogenetically-plausible evolutionary scenarios for music's emergence, cast in a co-evolutionary, niche construction perspective. In other words, this is to offer and assess hypothesised lineage explanations (Calcott 2009) of music: evolutionary narratives of the music crane (to borrow a metaphor from Daniel Dennett)—a phenomenon 'designed and built, from everyday parts already on hand' (Dennett 1995, p. 75) that then undergo evolutionary development and coalescence. (And once built, opens up further evolutionary options.)

Gary Tomlinson (2015) has recently attempted one such hypothesis, focusing on the evolution of key aspects of hominin musicality and providing a theory of their coalescence. Over the next two chapters I contribute to this multidisciplinary research programme by providing an alternative hypothesis of musicality's foundations and early developments. It is a foil in many ways to Tomlinson's narrative. I envision an earlier entry into a unique hominin socio-cognitive niche, and will attempt an explanation less reliant on what Tomlinson dubs "abstract machines". I build upon increases in Plio-Pleistocene hominin vocal musicality/communication and distinctively hominin cognitive competences, in co-evolutionary tandem with morphological and neural upgrades. My picture is situated generally in the framework of the social brain hypothesis, though co-evolution and niche construction feature in my model more prominently than in the standard line, as I will soon detail.

\section{Hominin socio-cognitive evolution}

According to the standard theory of hominin evolution, our ancestors diverged from the chimpanzee/bonobo ancestral lineage around 6-7 Mya. For the first few million years, our ancestors seem to have lived similar lives to that of their other great ape counterparts. This was to change, however, from around 4 Mya. 
Over the course of their evolution, our ancestors became obligate bipeds, toolusers and -makers, fire-harnessers, and eventually masters of language. They cooked their food, grew bigger brains, developed longer periods of juvenility and adolescence, and continued to be active members of their social worlds after menopause/fertility. They evolved symbolic cultures, religions, and artistic practices.

In what follows I detail this evolutionary narrative further, emphasising connections with musicality, though contextualised in the bigger picture of hominin evolution in general. Along the way I also offer some less "standard" perspectives on the hominin evolutionary career, building a model that synthesises the empirical work reported and highlights some challenges to received wisdom. In what follows I think through some possibilities that, in my view, deserve serious attention, and I reflect critically and methodologically on the human origins literature.

I am mindful that some order of speculation is involved in constructing evolutionary models and prospects for testing such models. Yet I have, in chapter 1, defended the general methodology and purpose of such research and I hope it is taken as intended: as a springboard for theorising about human evolution. Briefly put, it is a tentative model (by necessity — a great deal remains to be learned about human evolution and cognition), which attempts to integrate the material evidence, combined with what I take to be reasonable inferences, in order to narrate human evolution through a bio-cultural, niche construction, social-brain lens, with particular reference to the evolution of musicality and relevant selective pressures.

The human mind evolved to deal with profuse environmental and social information. A large part of what the human mind copes with is a complex social world within changing environments. Humans, and primates generally, live in more complex and socially demanding setups than any other mammals. As advocates of the social brain hypothesis rightly attest, considerations of sociality should be at the heart of discussions about hominin cognitive evolution (Gamble et al. 2014). ${ }^{69}$

\footnotetext{
${ }^{69}$ Dunbar (2014), chapter 3, provides a neat introduction to the social brain hypothesis. I further discuss this hypothesis in due course.
} 
Modern human brains are, when compared proportionally to body size, much larger than all other mammals including other primates (e.g., Dunbar 1996, 2008; Gowlett et al. 2012). Neocortex size and group size in primates are linked, implying an evolutionary relationship between increased sociality and encephalisation. But, to be sure, this is not the whole story, for humans have far surpassed the cognitive and cultural limitations of all extant great apes—humans put their big brains to use in rather unique ways. The cognitive capacities of our hominin ancestors have co-evolved with increased sociality and social learning environments. This is socio-cognitive niche construction.

Humans have evolved the capacities for inductive, deductive, speculative, and counterfactual reasoning; they have evolved the capacity for mental time-travel ${ }^{70}$, imagining being in the bodies and minds of other agents, fictive pretend play and mimesis, higher orders of intentionality/theory of mind, and so on. This set of traits distinguishes humans from the other great apes—that is, a large part of "being human", in the vernacular sense, comes from these cognitive aptitudes (Suddendorf 2013). Our lineage's unique kind of cognition co-evolved incrementally in the face of social and ecological pressures that I describe through six bio-cultural stages of our lineage's evolution. My hypothesis is that musicality, conceived as a mosaic of various elements (that I shall point to throughout), incrementally evolved and had far-reaching consequences. That's not to say that I claim it was a "prime mover" of hominin cognitive evolution: the combined complexity of socially and cognitively demanding pressures and feedback drove hominin socio-cognitive co-evolution and niche construction throughout the Plio-Pleistocene. Thus I shall also point to other developments: trackways reading, stone tool production, control of fire and so on, providing a richer picture. (This is not to downplay the importance of other advances also widely discussed in the literature, such as the evolution of cooperation.) In Table 7.1 I very briefly describe these stages, which I then expand upon throughout this chapter and the next.

\footnotetext{
70 "Mental time-travel" is the term for the cognitive ability to relive memories of past events and imagine future events— that is, imagining oneself in the elsewhen (see e.g., Debus 2014; Gerrans and Sander 2014).
} 
Table 7.1: Six key bio-cultural stages in hominin evolution

\begin{tabular}{|c|c|c|c|}
\hline & From & Communication/Culture & Salient hallmarks \\
\hline 1. Pre-Homo & $\begin{array}{l}\text { C. } 4 \\
\text { Mya }\end{array}$ & $\begin{array}{l}\text { Simple/opportunistic } \\
\text { ape-like sociality }\end{array}$ & $\begin{array}{l}\text { Foundations of musicality } \\
\text { Reactive, purposeful, discrete vocalisations } \\
\text { and upgrades in intentional listening. } \\
\text { Crude stone tools from } 3.3 \text { Mya. } \\
\text { Simple trackways reading emerges (by } 3.6 \\
\text { Mya). }\end{array}$ \\
\hline $\begin{array}{l}\text { 2. Oldowan } \\
\text { Homo habilis }\end{array}$ & $\begin{array}{l}\text { C. } 2.6 \\
\text { Mya }\end{array}$ & Mimetic/iconic culture & $\begin{array}{l}\text { Expansion of foundations of musicality } \\
\text { Increasingly continuous vocalisations in } \\
\text { various social domains; increases in top- } \\
\text { down control; incremental evolution of } \\
\text { unique cognitive niche; selection for neural }\end{array}$ \\
\hline $\begin{array}{l}\text { 3. Acheulean } \\
\text { Homo erectus } \\
\text { (sensu lato)/ } \\
\text { Homo ergaster }\end{array}$ & $\begin{array}{l}\text { C. } 1.8 \\
\text { Mya }\end{array}$ & $\begin{array}{l}\text { Gestural/indexical } \\
\text { culture }\end{array}$ & $\begin{array}{l}\text { and physiological changes. } \\
\text { Oldowan stone tools to Acheulean bifaces; } \\
\text { lithic sound play; vocal musicality. } \\
\text { Systematic trackways reading emerges. }\end{array}$ \\
\hline $\begin{array}{l}\text { 4. Late } \\
\text { Acheulean } \\
\text { From Homo } \\
\text { heidelbergensis } \\
\text { to archaic } \\
\text { Homo sapiens }\end{array}$ & $\begin{array}{l}\text { C. } 800 \\
\text { Kya }\end{array}$ & $\begin{array}{l}\text { Verbal-gestural } \\
\text { culture }\end{array}$ & $\begin{array}{l}\text { Proto-music } \\
\text { Incremental evolution of social proto- } \\
\text { music; vocal musicality provides the } \\
\text { medium for subsequent evolution of verbal } \\
\text { proto-language (from principally gestural } \\
\text { "showing how" communication). } \\
\text { Fire control, more complex (e.g., hafted) } \\
\text { tools. } \\
\text { Speculative trackways reading emerges. }\end{array}$ \\
\hline $\begin{array}{l}\text { 5. Mid/Upper } \\
\text { Palaeolithic } \\
\text { Homo sapiens } \\
\text { sapiens }\end{array}$ & $\begin{array}{l}\text { C. } 250 \\
\text { Kya }\end{array}$ & $\begin{array}{l}\text { Mythic culture and } \\
\text { "Behavioural } \\
\text { modernity" }\end{array}$ & $\begin{array}{l}\text { Full-fledged music; musical instruments } \\
\text { Changes in social organisation and } \\
\text { concretisation of high-fidelity social } \\
\text { learning. } \\
\text { More complex musics and spoken } \\
\text { languages evolve; symbolism; artsy and } \\
\text { ritual activities. } \\
\text { Sophisticated musical technologies } \\
\text { evidenced at } 40 \text { Kya. }\end{array}$ \\
\hline
\end{tabular}




\begin{tabular}{|c|c|c|c|}
\hline $\begin{array}{l}\text { 6. Holocene } \\
\text { Homo sapiens } \\
\text { sapiens }\end{array}$ & $\begin{array}{l}\text { C. } 10 \\
\text { Kya }\end{array}$ & $\begin{array}{l}\text { From sedentary } \\
\text { settlements and } \\
\text { agriculture, } \\
\text { to modern cities, } \\
\text { to extra-terrestrial } \\
\text { voyage }\end{array}$ & $\begin{array}{l}\text { Music's continually-evolving career } \\
\text { Changes in economic and social } \\
\text { organisation; rapid cultural evolution and } \\
\text { technological development in all domains. } \\
\text { Reinforcement mechanisms for cultural- } \\
\text { informational archives. } \\
\text { Emergence of "musicians-as-experts", } \\
\text { whose job it is to perform to more passive } \\
\text { (and elite/hierarchical) audiences- } \\
\text { abstracting music (as sound) from its social } \\
\text { context. } \\
\text { The "release" of music from musicality: } \\
\text { music in its current modern form as } \\
\text { decoupled art object. }\end{array}$ \\
\hline
\end{tabular}

Five caveats before I begin. First, although conceived as a kind of lineage explanation, I attempt an account that tracks and links series of individual phenotypes and feedback, rather than developmental systems/mechanisms (cf. Calcott 2009). I aim it to be compatible and consistent with contemporary interpretations of the evidence at hand (in ways I shall point out), as well as empirically constrained and tractable, so as to move from "just so" speculation to "how plausibly" scenario building-that is, not reliant on "magic bullets", "skyhooks", or low probability events. Needless to say, any account of this kind must be put forward tentatively and revised as new evidence and interpretations come to light: there is still some speculation involved. So more work is required to link some of the conjectures to testable hypotheses. Nonetheless, it is my contention that plausible narratives have practical utility and are thus not simply unproductive distractions (at least insofar as their questionable testability is concerned). ${ }^{71}$ Explanations do not emerge from data alone; human insight is required to develop them. Historical science proceeds in a plurality of ways (Currie 2016b) —historical scientists come to know more about the past through evaluating hypotheses/scenarios against consilience of evidence from multiple sources, dependency relations, and "smoking guns" (trace evidence highly suggestive of a

\footnotetext{
${ }^{71}$ For a richer methodological defence of evolutionary scenario-building on this score, see Sterelny (2012a).
} 
hypothesis). And one such avenue available for philosophers to contribute is to develop scientifically informed scenarios of this kind (that is, lineage explanations). The key phrase here, as Tuttle puts it, is scientifically informed. That is: 'Persuasive paleoanthropological scenarios differ from science fiction in that the former is bounded by facts' (Tuttle 2014, p. 4). The bottom line is that I offer a plausible answer to questions about which we just do not yet know. And narratives such as mine have the potential to uncover connections that may have not been clear, guiding subsequent empirical testing and the search for new evidence, research methods, and conceptual/methodological frameworks.

Second, I should add that much of my discussion concerns the palaeoanthropological and archaeological records, and associated implications and inferences. This is in contrast to some theorists working on the evolution of music that focus on evidence from contemporary developmental studies (developmental psychology and biology), for example, Trehub (2001, 2003), and from neuroanatomical studies in contemporary humans, such as the connections and overlap between music, language, and the brain, for example, Patel (2008). As Sterelny (2016b) notes in the case of evolution of language, evidence of this kind is surely suggestive-and will be mentioned in relevant places throughout my discussion (and, indeed, has been mentioned in chapter 6 and elsewhere in this thesis) — but since human brains are profoundly plastic, on both ontogenetic and phylogenetic timescales, I am hesitant to rely too heavily on this evidence in evolutionary scenario building (Malafouris 2010).

However, this is not to suggest that all the evidence and components of my model are epistemically equivalent. Throughout I will flag differences in epistemic status directly or indirectly: that is, what we know from the record, what we have more modest evidence of, and what is constrained speculation. Even so, as Robin Dunbar puts it, the following 'will be an exercise in detection. We have the crime scene before us in the archaeological record, tantalisingly imperfect as every crime scene always is. Our task is to infer what happened where, when and why... We shall proceed, like all good detectives, by trying to fit the bits of the jigsaw together' (Dunbar 2014a, pp. 30-31), building a step-by-step, cumulative and dynamic picture through evolutionary time. 
Third, I am aware that there will be much more to the story than I can cover here. My evolutionary narrative is certainly not exhaustive; omissions are unavoidable. But as Lewis-Williams (2004) remarks, evolutionary scenarios do not have to explain everything in order to explain something.

Fourth, I should be clear that the stages of the scenario are not conceived as sudden shifts, but intended to capture what is incrementally evolving throughout that stage. Dividing hominin evolution into phases is a convenience not meant to entail saltationism. The endpoints of each stage should thus represent a tighter chronological benchmark for the capacities discussed in that stage than their starting points. Similarly, for convenience, I appeal to C. S. Peirce's semiotic distinctions (icon, index, symbol) in my characterisation of the evolution of hominin communication. The application of this set of distinctions in evolutionary thinking is a reasonably common strategy (for an example in the context of evolution of music, see Tomlinson 2015), although this common strategy has recently been criticised by Godfrey-Smith (2014b). ${ }^{72}$

Finally, note that right from the beginning, stage 1 hardly resembles anything musical. But since evolving phenomena are based on earlier forms, evolutionary accounts should reflect this. (For example, consider the first stage of religion—called "embodied religion"-in Kim Sterelny's (2017b) evolutionary model, which comprises a collection of salient artsy practices like music, dance, storytelling, as well as cultural rituals such as initiation ceremonies. There is no mention of god or spirit worship or anything distinctively religious by today's standards.)

\section{Hominin evolution and the foundations of musicality}

In my view, human musicality has deeply entrenched and very ancient foundations. Allow me to paint a picture of the social and cognitive life of pre-Homo at my narrative's starting point, beginning in Africa around 4 Mya with our social, omnivorous pre-Homo forebearsmobile, non-tree-nesting, habitual bipeds. ${ }^{73}$ These ancients foraged in expanding, changing

\footnotetext{
${ }^{72}$ That said, mine is a loose application of these distinctions, not intended to precisely demarcate categories of signs, as per Peirce, but rather as a heuristic for describing communicative aspects in broad strokes (in particular the sociality of communication across stages-what I mean by this will become clear in due course), so I do not think my model is subject to Godfrey-Smith's apprehensions. ${ }^{73}$ Bipedalism kicked in at 5 Mya at the latest (Dunbar 2014).
} 
environments, including open lightly wooded savannah areas, and the treeless wetlands full of tall sedges and shrubbery within those regions. ${ }^{74}$

Bipedalism itself had three crucial upshots (following Sterelny 2014). Firstly, bipedalism evinces the ecological shift of our pre-Homo ancestors, from arboreal lifeways to less tree-based environments. Secondly, bipedalism liberates hands from the job of locomotion, allowing our ancestors to pick up and carry food, infants, and, in due course, stone tools, leading to increased manual dexterity for complex tool manufacture, food processing, and so on through co-evolutionary feedback. Thirdly, bipedalism 'forced our ancestors to sleep on the ground, thus making them more vulnerable to predators at night' (Sterelny 2014, p. 265). Large cats and other predators would have posed a genuine threat to our hardly-formidable ancestors of this period. So it is safe to assume that they had to band together at night for group defence-imagine loud, intimidating vocalisations, volleys of thrown stones, and so on-so they learned to better tolerate conspecific proximity and increased group size, selecting for increased tolerance to the physiological costs of group living. The observation that flakes sometimes chipped off stones when thrown, and were useful for cutting/slicing, may have led to the early beginnings of lithic technology. Indeed the oldest known crafted stone tools date back to 3.3 Mya (Harmand and Lewis et al. 2015; Balter 2015).

It also seems that the socio-ecological niche of our pre-Homo ancestors, described above, triggered simple conspecific trackways reading and use (that is, follow-the-leader reiteration of footprint tracks) for safety and orienteering. Shaw-Williams (2014) presents evidence that this was occurring by 3.66 Mya, as I shall discuss in due course. Shaw-Williams argues that since our ancestor's bipedal footprints were easily recognisable they became social markers, which triggered selection for the kind of decoupled cognition needed to glean the large amounts of social information contained in conspecific trackways. And no other great ape uses tracks as natural signs.

Now, as hinted earlier, my narrative draws on the social brain hypothesis (e.g., Gamble et al. 2011, 2014; Gowlett et al. 2012); that is, the idea that explicating the complex

\footnotetext{
${ }^{74}$ See Finlayson et al. (2011) for evidence of a mixed woodland-savannah, wetland, and rocky habitat environment. Also see Bender et al. (2012) for a full historical review and a new synthesis of open savannah and wetlands hypotheses.
} 
social worlds of hominin ancestors is vital to understanding hominin cognitive evolution: hominin brains are social brains, and sociality was a key driver of encephalisation (though for Gamble, Gowlett and Dunbar it is the key driver). This idea and the research scaffolding it provides me with both a key framework and methodological motivation:

The social brain hypothesis encourages us to think in terms of a domain rather than isolated strands of evidence. If we are able to understand the process of human evolution as one where social cognition developed through interactions of hominins with each other and with materials, artifacts, and landscapes, then the contexts where social life took place become central to the study. (Gowlett et al 2012, p. 701.)

Importantly, as I will explain, more efficient intentional forms of communication are hypothesised to have upgraded methods of socio-psychological grooming throughout the evolutionary career of our ancestors, as suggested by Robin Dunbar (e.g., see his 1996, 2004); consider here one individual vocalising to several others. Gowlett et al. (2012) stress the importance of proto-music for managing more intense social relating, and so do $\mathrm{l}$. However I part from the standard line on a few scores. The first has to do with the evolution of theory of mind. In my view, an earlier entry into a unique socio-cognitive niche was required to give our social complexity this kick start. Hence, when I introduce my model, I do so with our pre-Homo, ape-like ancestors from around 4 Mya onwards, allowing for a more incremental evolution of theory of mind capacities. Second, I find a much more central role in my model for co-evolution and niche construction than the standard line, as will become clear throughout my narrative. On my view, encephalisation is not merely an adaptive result selected for by social complexity (cf. Gamble et al. 2011). Indeed I have pushed for a dynamic picture of hominin evolution in the preceding chapter. Third, for my purposes I worry not about explicating the precise nature of the correlation between brains and groups-matters that are open for debate that would take me too far afield-although needless to say, it appears modern humans fit cleanly into the projected line on group size 
and neocortex volume correlations in great apes (Gamble et al. 2011, 2014), evidence enough for a strong evolutionary connection between sociality and cognition in our lineage. Gamble et al. put it like this:

The fact that community size correlates closely with neocortex size in primates and modern humans immediately gives us a handle on estimating likely community sizes of our fossil ancestors. The logic is very simple. If community size in monkeys and apes correlates with brain size, and modern humans sit exactly where they should do on the same regression line, then all fossil hominin species must lie between these two points-unless, of course, our fossil ancestors behaved in such a completely different way that they do not fit the general pattern for all other primates, including modern humans. That is so implausible it barely deserves serious consideration. (Gamble et al. 2014, pp. 73-74).

This being said, due caution is required nonetheless, as averages may change when more specimens are unearthed. In any case, for my purposes the exact numbers are not important; it's the general trend that is salient (that is, I need not commit myself to a simple covariation). Table 7.2 provides an approximate guide to hominin brain size evolution and group size growth reconstructed from data discussed in Dunbar (2014a) and Gamble et al. (2011). The important thing to note at this stage is that as group size increases, new social methods for cohesion and bonding need to develop to manage social relations, ease social tensions and other costs of larger group living. I will be detailing this further, focusing on the role of musicality in this regard. 
Table 7.2: Brain size/group size model predicted by social brain hypothesis

\begin{tabular}{|c|c|c|c|}
\hline & Stage & $\begin{array}{l}\text { Average brain size } \\
\text { (cubic centimetres; } \\
\text { approx.) }\end{array}$ & $\begin{array}{l}\text { Predicted group } \\
\text { size (approx.) }\end{array}$ \\
\hline Australopithecines & $\begin{array}{l}\text { Pre-Homo } \\
\text { (Stage 1) }\end{array}$ & $\begin{array}{l}480 \mathrm{cc} \\
\text { (Roughly that of } \\
\text { chimpanzees) }\end{array}$ & $\begin{array}{l}50 \\
\text { (Roughly that } \\
\text { of chimps) }\end{array}$ \\
\hline H. habilis & $\begin{array}{l}\text { Oldowan } \\
\text { (Stage 2) }\end{array}$ & $\begin{array}{l}620 \mathrm{cc} \\
\text { (increase of } 29 \% \text { over } \\
\text { australopith/chimp } \\
\text { baseline) }\end{array}$ & {$[\sim 62.5]$} \\
\hline H. ergaster & $\begin{array}{l}\text { The Oldowan/Acheulean } \\
\text { cusp; early Acheulean } \\
\text { (Stage 2-3) }\end{array}$ & $\begin{array}{l}760 \mathrm{cc} \\
\text { (increase of 58\% over } \\
\text { australopith/chimp } \\
\text { baseline) }\end{array}$ & 75 \\
\hline H. erectus & $\begin{array}{l}\text { Acheulean; Acheulean/Late } \\
\text { Acheulean cusp } \\
\text { (Stage 3-4) }\end{array}$ & $\begin{array}{l}930 \mathrm{cc} \\
\text { (increase of } 22 \% \text { over } \\
\text { ergaster) }\end{array}$ & 100 \\
\hline H. heidelbergensis & $\begin{array}{l}\text { Late Acheulean } \\
\text { (Stage 4) }\end{array}$ & $\begin{array}{l}1,170 \mathrm{cc} \\
\text { (increase of } 54 \% \text { over } \\
\text { ergaster) }\end{array}$ & 120 \\
\hline H. sapiens sapiens & $\begin{array}{l}\text { Anatomically and } \\
\text { behaviourally modern } \\
\text { humans } \\
\text { (Stages } 5 \& 6 \text { ) }\end{array}$ & $\begin{array}{l}1,370 \mathrm{cc} \\
\text { (increase of } 17 \% \text { over } \\
\text { heidelbergensis) }\end{array}$ & 150 \\
\hline
\end{tabular}

Time now to outline my narrative. In the following, I present the basic picture, briefly and schematically. I fill in the details throughout the remainder of the thesis.

While aspects of the social brain hypothesis form one part of my socio-cognitive niche-construction story about musicality, aspects of Kim Shaw-Williams's social tracking theory form another-although perhaps more auxiliary—part of my story. Entering the unique social tracking niche rehearsed theory of mind skills, paving the way for our lineage's extraordinary social learning and cultural niche construction, as our ancestors 
incrementally increased their own cultural and social complexity. (For more on the concept of cultural niche construction see e.g., Laland and O'Brien 2011; Odling-Smee and Laland 2011.) My focus, though, is the ancient origin of human musicality itself, and both trackways reading (which requires intentional control of gait, rehearsing some degree of intentional interval-based timing) and increased vocalisation were responses to our Pliocene entry into a very demanding yet resource-rich socio-ecological niche described above.

I discuss six cumulative stages (see Table 7.1 above). The first stage of the narrative (briefly described already) is designated as pre-Homo sociality. Here we see evidence of intentional tool use and trackways reading, and perhaps evidence of cooperative (though, presumably opportunistic) hunting. I discuss some plausible advances in musicality during this time, though this stage of the narrative is of necessity put forward more tentatively. (The material record of this early stage is far sketchier than later stages.)

The second stage, beginning roughly 2.6 Mya, is the Oldowan, and indeed it is much more archaeologically visible. For example there is much more solid evidence of the kinds of activities that suggest intentional cooperation. I judge the communication level of this stage to be mimetic/iconic, similarly to Merlin Donald (2001).

The third stage, in place by around 1.8 Mya (Lepre et al. 2011), is gestural/indexical Acheulean culture (not replacing but adding to the mimetic/iconic culture of the Oldowan). Again, here we see evidence of increasing intentional competences and forward planning in multiple domains (e.g., hunting and raw material transport). From the more sophisticated Acheulean toolkit, we can infer that social learning had also advanced. In my view, the incremental emergence of intentional gesture from mime and Oldowan "showing how" pedagogy was seamless, as was the emergence of intentional musicality and sound play.

The fourth stage, through roughly 800 to 250 Kya, is Late Acheulean verbal-gestural culture (again, bolstering that of the previous stage). During this stage a final rapid surge in encephalisation occurred (see Table 7.2). Remarkable systematic/speculative tracking and hunting skills are evident by around $500 \mathrm{Kya}$, as is the beginnings of a proto-aesthetic sense. I argue that there is good reason to think our big-brained, social Heidelbergensian ancestors became proto-musical during this stage. And I suggest that intentional vocal musicality—singing, chanting, crooning, humming —in tandem with increasingly complex 
gestural communication and anatomical co-evolution, enabled the subsequent emergence of spoken proto-language.

The fifth stage is from roughly 250 Kya. Merlin Donald (2001) calls it "mythic" culture. Here we have arrived in the Mid/Upper Palaeolithic, the long stretch of the evolution of behavioural modernity-hominins with full language, autobiographical minds aware of their inevitable demise, cultural origin myths, musicians and instrument makers, story-tellers and shamans, long distance trade/exchange networks (of up to 300 kilometres; see Marwick 2003) and all the rest.

Finally, from around $10 \mathrm{Kya}$, the sixth stage-beginning with the Neolithic transition—saw the rapid explosion and cultural evolution of musics. Many human lifeways shifted to permanent, sedentary, agricultural, and increasingly hierarchical societies. The human career from the Neolithic transition onwards is characterised by changes in economic organisation and power distribution, full division of labour, skill specialisation, an eruption of technologies (including, as I shall detail, more complex musical instruments), and high-fidelity social learning (including the emergence of writing systems). All this would eventually give rise to music's current form—something that experts specialise in after years of training, which they perform to a passive audience of consumers-abstracted from the expression of musicality still evident in a more basic form in all typical, normallydeveloped humans today.

\section{Stage One: the trackways-reading and tool-making pre-Homo}

As I have said already, by 4 Mya our pre-Homo Pliocene ancestors stopped tree-nesting and took up foraging for increasingly higher quality diets in Africa's expanding, open lightlywooded savannah environments and the wetlands and regions of tall sedges and shrubbery within.

Environmental change was profuse at the start of the Pliocene throughout the East African Rift (WoldeGabriel et al. 2000, 2001), and development of new social behaviours was required to achieve optimal non-arboreal, mobile foraging. In my view, three important social behaviours took hold as part of this lineage-defining change. 
First, vocal communication increased. That is, I hypothesise that non-arboreal mobile foraging would have triggered an increase in automatic/reactive vocalisation use for inter-conspecific communication, such as calls to assist band members lost or lagging behind (or foraging out of sight of core group). In general, like carnivores (and unlike herbivores), omnivores need to be wide-ranging in order to find and catch their often seasonally mobile prey. ${ }^{75}$ (And note, for instance, that extant human foragers have huge ranges compared to those of chimpanzees.) Wide-ranging navigation usually results in periods of limited conspecific visibility, which would make vocalisation a vital form of communication for our Pliocene ancestors, as well as an adjunct to group defence. When our Pliocene ancestors gave up tree-nesting, maintaining auditory contact with conspecifics during the day and banding together at nightfall for group defence became essential behaviours. ${ }^{76}$ Our Pliocene ancestors plausibly resorted in loud, intimidating vocalisations, volleys of thrown stones, and so on, to deter predators such as large cats. And perhaps this strategy was used to drive predators from kills, in "mob scavenging” style. Stealth/ambush predators like leopards cannot afford to be seriously injured since even a broken leg could be a death knell. Shards chipping off flung stones and the observation that those shards are useful for cutting/slicing the coveted carcass may have led to the early beginnings of lithics (see below).

The details here may be conjectural but the crucial point is that increases in vocalisation very plausibly became a vital aspect of ancient Pliocene sociality. Given the morphology of our ancestors at this time, these vocal calls would have been shorter, more detached, and of a limited pitch range compared to that of modern humans-and admittedly not particularly musical. Nevertheless, for non-tree-nesting primates, finding the band at night for group defence was important, and lost or laggard band members could hone in on discrete calls vocalised by the band at nightfall. Vocalisations would also make their lost author's position in, for example, more-than-head-high wetland-edge vegetation easier to locate (from a non-arboreal, ground-based perspective).$^{77}$ If the general picture is approximately right, and there is no reason to think it isn't, we would probably expect

\footnotetext{
75 Obviously in times of scarcity herbivores need to be wider ranging than normal, and so on.

${ }^{76} \mathrm{Keep}$ in mind the poor night vision and olfactory sense of great apes compared to that of most predators.

${ }^{77}$ And perhaps also advertising territorial occupation, like howler monkeys, though again perhaps not, if the territory sizes in question were simply too large for that to be effective.
} 
selection for coordination between voices to occur, selecting for increases in top-down control over vocalisation.

The second social behaviour, social trackways reading, is explicated by ShawWilliams (2014). The idea is that the mobile socio-ecological niche of our Pliocene ancestors triggered simple conspecific trackways (footprint) utilisation, initially for safety and orienteering. That our pre-Homo ancestors began reiterating conspecific footsteps in a follow-the-leader, associative manner is demonstrated by the fossilised footprints dated to 3.66 Mya at Laetoli-a 30 meter trail of side-by-side trackways of two individuals set in ash, with one of the pairs of footprints consistently containing a nested pair of footprints, of some third individual (see Figure 7.1, overleaf; also see Leakey and Harris 1987; Crompton et al. 2012; Renfrew and Bahn 2012).

Shaw-Williams argues that social tracking plausibly increased our ancestor's fitness because mobile obligate bipeds are severely impaired by damage to either of their feet. It is costly to continuously check where one is walking especially in unfamiliar environments replete with other potential threats and distractions. And even familiar environments are changeable enough and can hide or superficially cover all sorts of dangerous bog-holes, quicksand, and sharp objects. More importantly for my purposes, stepping in the footprints of another individual in such a precise manner as demonstrated in the long Laetoli trail (there is only one botched attempt in which the footprints were not well placed one within the other) is greatly assisted by deliberately measuring out or rhythmically attuning one's walk—rather than walking in one's usual and natural/unintended gait—given the different gait and leg length of the individual whose tracks one is stepping in. We would expect many more botched attempts if this were not occurring. Maintaining a gait and walking speed other than what is natural requires top-down, executive control (Kahneman 2011), as Mary Leaky and her team found out when they tried following each other's tracks back at camp after the trail's discovery (Leaky and Harris 1987). I think this has important upshots for explicating hominin entrainment, one key constraint on a theory of music's evolution (Merker et al. 2015). 


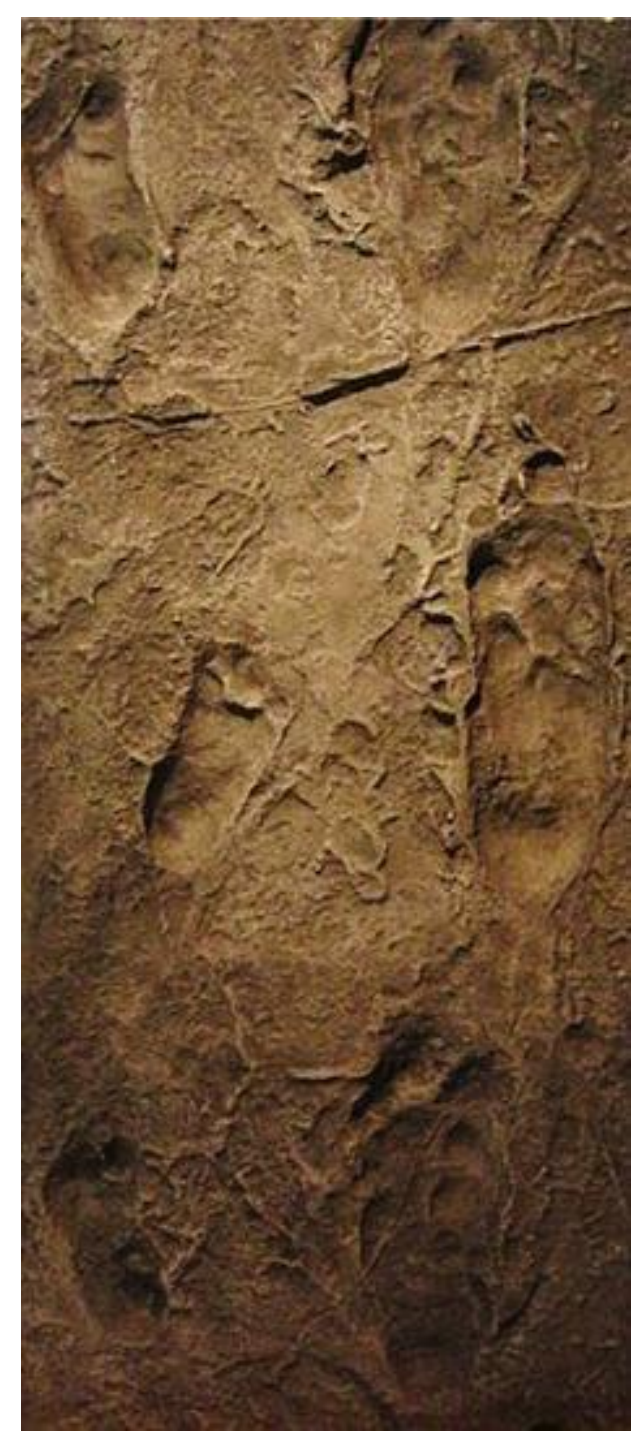

Figure 7.1: Laetoli footprints replica ${ }^{78}$

\footnotetext{
${ }^{78}$ Replica of the Laetoli fossilised footprints of 3.66 Mya, clearly displaying juvenile's prints on the left and prints of two adults on the right, showing the second adult is reiterating the first's footprints. Bottom right "dual print" indicates a double attempt by the second adult (left foot). One can see that their feet possessed arches and human-like (large and adducted) big toes: they were obligate bipeds. There are no hand- or knuckle-prints, which would have been left behind by the likes of an ancestral chimpanzee.

Photo by "Momotarou2012", freely distributable and adaptable under Creative Commons Attribution-Share Alike 3.0 Unported licence. Source= http://en.wikipedia.org/wiki/File:Laetoli_footprints_replica.jpg. Accessed 20/08/2016.
} 
Musical entrainment, or beat-based synchrony, is a crucial ingredient in human music making. Merchant and Honing (2014) distinguish interval-based timing cognition from beat-based timing cognition. Interval-based timing (internal time-interval regulation: interval reproduction, categorisation and interception) is shared by non-human primates (Zarco et al. 2009), with beat-based timing (external beat synchronisation: rhythmic entrainment, synchronisation and continuation) peaking in humans (among primates), building on basic primate interval-based timing. Although wild great apes drum on their bodies, on tree buttresses and the like, these displays do not exercise beat-based synchrony. However some rare and not-yet-replicated instances of beat-based timing have been observed in chimpanzees in captivity (Dufour et al. 2015; Hattori et al. 2013), suggesting that (some) chimps may have a latent sensitivity to external pulse, indeed possibly only enabled by the cognitive offloading associated with captivity. At the very least, this shows it is safe to presume that a more-than-rudimentary capacity for time-interval regulation was also present in the Pan-Homo last common ancestor. In my view, it seems that this would have enabled the precision of trackways following evident in the Laetoli nested tracks. Social tracking - in particular, stepping in the prints of another individual—rehearsed intervalbased timing cognition, bringing internal time-regulation (and associated motor-regulation) under voluntary control. (Or at least, being a major contributing factor.) Social tracking thus appears to bear witness to an earlier increase in intentional voluntary control than what is usually assumed of our Pliocene ancestors. And not just voluntary control: voluntary control of a regular, repeated series of acts.

Intentional voluntary control is a cognitive adaptation and I see the long evolution of cognition as undertaking two salient (though incremental) upgrades, the first of which is already well underway at this stage in the narrative. Following Liebenberg (1990), ShawWilliams discusses three levels of trackways reading skills, simple, systematic, and speculative, and I will borrow this tripartite framework for conceptualising hominin sociocognitive evolution.

The first upgrade saw the expansion from the long and incremental career of simple cognition (reactive, automatic cognition; often dubbed "System 1" cognition) homologous with other primates, to simple + systematic cognition (incrementally evolving intentional, top-down, voluntary control and attention, impulse control, rudimentary theory of mind, 
basic forward planning). ${ }^{79}$ This incremental evolutionary transition must have been well underway by the time of the Lateoli tracks. It is evident, to some degree, in non-human great apes today: chimpanzees and bonobos for example are capable of intentional gesture, rudimentary theory of mind, and so on. ${ }^{80}$ (So the early hominin lineage saw an expansion of these capacities, but not their great ape origin.)

The second incremental upgrade will come later, that is, the upgrade from simple + systematic cognition to simple + systematic + speculative cognition (enabling mental timetravel, hypothetical reasoning, complex theory of mind and forward planning, and so on; that is, full "System 2" cognition), which I will argue was in place by Homo heidelbergensis in the Late Acheulean. (These two upgrades may well be differences in degree, not dramatically different kinds of cognition.)

Finally, the third social behaviour is tool production and use. Given that we now have what appears to be cut-marked bones from a mammal as large as a cow dated at 3.4 Mya (McPherron et al. 2010), with the nearest suitable source of stone $6 \mathrm{~km}$ away-and intentionally produced stone tools from 3.3 Mya (Harmand and Lewis et al. 2015; Balter 2015) - there was at least one fairly smart and cooperative pre-Homo species living during the Mid-Pliocene.

These ancient tools were discovered in Kenya at Lomekwi 3, west of Lake Turkana, around 1,000 km from Olduvai Gorge. Researchers 'uncovered nearly 20 well-preserved flakes, cores, and anvils apparently used to hold the cores as the flakes were struck off, all sealed in sediments that provided a secure context for dating. An additional 130 pieces have also been found' (Balter 2015, n.p.). Tool analysis revealed that the tool-maker rotated the stone core while striking it to create flakes-the same manufacturing method as that of the tools of the Oldowan.

\footnotetext{
79 For the dual system theory of the mind (System 1/System 2 cognition) see Evans (2003); Kahneman (2011).

${ }^{80}$ See for example the research of Michael Tomasello and collaborators: e.g., Call and Tomasello (2008).
} 
The 3.4 Mya cut-marked bones is tantalising evidence of tool use. ${ }^{81}$ Although acquiring carcasses like the one at 3.4 Mya might have been opportunistic rather than planned, the capacity to cooperatively carve large carcasses, as well as make and carry the stone tools/flakes necessary, indicates a far more complex sociality than is exhibited by chimpanzees and other primates.

We do not know for sure if our direct lineal ancestors were the makers/users of these stone tools (or the cause of the cut-marked bones). However, it is plausible, given the continuity between the Lomekwi tools and those of the Oldowan. If our pre-Homo lineal ancestors did in fact use stone tools, this obviously means that the capacity, at least, for stone tool production and usage was already established right at the start of the genus Homo, which emerged around 2.6-2.8 Mya (Gibbons 2015; Villmoare et al. 2015). Indeed, before the 2015 discovery of the tools dated to 3.3 Mya, Peter Hiscock (2014) argued that the earliest Oldowan stone tools were made skilfully, suggesting an undetected pre-Homo history of tool-making. It looks like he was right. Note that general utilitarian use of found objects is most likely a homology: after all, chimps can be excellent tool users (e.g., sticks, long grass-stems, and wooden "spears")—consider termite fishing. As I have already mentioned, the use and production of stone tools could have very easily and straightforwardly developed from thrown volleys of stones in defence from predators or driving predators from kills, so our pre-Oldowan, pre-Homo ancestors may have noticed that shards sometimes flaked off, and were useful for cutting/slicing, marking the beginnings of lithic technology in this pre-Homo stage. And note that flake extraction has been observed in chimpanzees in experimental lithic/resource-exploitation contexts (see e.g., Carvalho et al. 2008).

What of pre-Homo musicality here? In my view, opportunistic cooperative behaviours like carving large mammals and group hunting techniques would have been associated with group chanting of recognisable vocal patterns (consider the vocalisations of highly social mammals, such as group-hunting orcas and wolves, for instance: e.g., Ford 1991; Gazzola et al. 2002; Gese and Ruff 1998; Harrington 1989—or the vocalisations of the cooperatively territorial or cooperatively breeding social New World primates: e.g., Kitchen

\footnotetext{
${ }^{81}$ There is some controversy surrounding these bones as evidence of tool use (see e.g., DomínguezRodrigo et al. 2012) so for now this must be taken as suggestive, not conclusive.
} 
et al. 2015; Borjon and Ghazanfar 2014). This would coordinate group action and would save energy-consuming confrontations with other bands. And as a kind of proto-haka-so named after a New Zealand Māori form of vocally-aggressive greeting/challenge—chanting could have been a warning to other bands in the near vicinity. This conjecture is not wild: chimpanzees too 'produce long range calls [and] also combine drumming and loud vocalisations in coordinated group displays that appear to play an important role in agonistic intergroup relations' (Hagen and Hammerstein 2009, p. 299). Falk, for one, suggests that 'australopithecines probably did a certain amount of chorusing and drumming, similar to African great apes' (Falk 2000, p. 211). And as Hagan and Bryant (2003) note, musicality can manifest as a signal of social cohesion. This scenario is consistent with cooperative hunting and gathering in another way: it saves ineffectual foraging in areas where other bands, or members of one's own band, have already plundered more easily obtained resources (shellfish and bird's eggs, for instance).

Trackways following and the foundations of vocal musicality both emphasise and rehearse intentional listening-top-down, deliberate, self-directed attention to sound (as contrasted with automatic, bottom-up taking in of sounds)—which, in my view, must be a prerequisite for evolving more complex musicality down the line. When tracking, especially in contexts of limited visibility, listening closely for auditory clues-and often deliberately not making sound (which perhaps selects for synchronised walking, see Larsson 2014)—are crucial skill-set upgrades. So complex sensitivity to sound was adaptive. Of course, some rudimentary form of intentional listening may well be very ancient, if the auditory vigilance of some predator/prey species counts as intentional listening for danger or a target. ${ }^{82}$ Yet our ancestors seem to be the only great apes that took the evolutionary route to more systematic intentional listening. And not just intentional listening, but intentional response to listening. (Compare with the famous reactive/automatic vervet monkey alarm calls: distinct calls for a bird of prey, snake, and leopard, of which each triggers a reactive/ automatic avoidance strategy: see e.g., Seyfarth and Cheney 1990). Only once more

\footnotetext{
${ }^{82}$ For instance, chimpanzees sometimes hunt monkeys, and might utilise top-down control of listening when doing so; this may also be true of stealth/ambush predators like leopards. And some prey species might intentionally listen if they are anxious about danger: some certainly intentionally scan visually. I still take it that humans have developed this intentional ability, which is a prerequisite for the evolution of musical traditions (required for musical evaluation), as a matter of degree, far beyond that of any other great ape.
} 
advanced systematic intentional listening emerged - since merely making a noise is far too easy and very often unintentional—could music and language begin to incrementally evolve. At least to some important extent, the contact and coordination calls that I described above moved beyond that of largely reactive vocalisations. In contrast, consider the reactive vocalisations of other apes. Bonobos have various combinations of pant-hoots that indicate both food presence and preference; chimpanzees may call to conspecifics if they have located good food; these calls can guide the foraging of others (Clay and Zuberbühler 2011, 2013).

That said, there is some tantalising evidence that chimpanzee top-down control of vocalisation is less rudimentary than has been supposed (see Slocombe and Zuberbühler 2007; Crockford et al. 2012; Fitch and Zuberbühler 2013). So, presumably, there is some basic form of intentional vocal control, and response to vocalisation, already present in the PanHomo last common ancestor. Our ancestors were expanding these capacities already present in rudimentary form. The territory-defence and mate-bonding vocalisations of the gibbons, on the other hand-the most musically sounding of our Hominoid cousins (though the furthest removed)—are not considered to be evidence of intentional listening (see Geissmann 2002).

Detecting and reacting to sound are ancient animal capacities, which confer obvious adaptive value; certain sounds can betray the presence of water, predators, conspecifics, food sources (e.g., bee-buzzing indicating honey), and so on. Some animals, whether predator or prey, demonstrate acute acoustic vigilance by tuning in to the soundscape of their environments, listening out for signs of danger or a potential meal. Our ancestors built upon this capacity by bringing it under intentional control, up-scaling its adaptive value, as alluded to in my discussion above. Intentional listening became systematic rather than merely opportunistic. And our ancestors certainly seem to be the only great apes that have taken the route to advanced systematic intentional listening.

In sum: I hypothesise that this early stage in our evolutionary career saw increases in vocal communication (contact and coordination calls), increased executive control, rehearsal of interval-based timing, and a shift towards intentional listening and increased sensitivity to sound. At some point, the production of these contact and coordination calls 
moved beyond reactive vocalisation, coming under executive control. Indeed, as I have pointed out, this scenario would plausibly select for coordination between vocalising individuals and increased vocal control, enabling better regulation of, say, signal strength (i.e., volume of voice), and thus co-evolutionary feedback. As we shall see, all this will undergo further development in the next stage of the narrative.

\section{Stage Two: the Oldowan}

Recent fossil evidence suggests that the genus Homo emerged around 2.6-2.8 Mya (e.g., Gibbons 2015; Villmoare et al. 2015). Thus this stage marks the initial evolution of our bigbrained, distinctively hominin ancestors. It was a period of massive encephalisation. Our Oldowan ancestors had brains roughly 30\% bigger than that of their australopithecine forebears. And throughout the Oldowan, our ancestor's brains would get another $30 \%$ bigger. (See Table 7.2 above; also see Antón and Snodgrass 2012.) Brain size increase points to new cognitive resources. Neural tissue is expensive to grow-as is both maintaining neural readiness and the firing of neurons-evincing upgrades in diet and foraging skill (including the use and production of lithics), but also greater social relating and cooperation (such as allocare: see Antón et al. 2014). These factors among others created a developmental niche which scaffolded hominin flourishing as well as a feedback loop which selected for the co-evolution of incrementally increasing cognitive capacities and social complexity, that is, Homo socio-cognitive niche construction. Following Merlin Donald (2001), I broadly define the next million years or so of Oldowan lithic culture as mimetic/iconic "showing how" culture ${ }^{83}$, rehearsing and expanding the rudimentary theory of mind capacities already present. Following Larsson (2015), I favour the view that pantomimic "showing how" for tool production and usage were adaptive developments in hominin sociality and the evolution of gestural communication.

The Oldowan saw the expansion of the social brain (e.g., Gamble et al. 2011; Gowlett et al. 2012). Cooperation and more-than-rudimentary theory of mind, backed up by

\footnotetext{
${ }^{83}$ Mimetic/iconic signals resemble (and thus "show") the whole or part of their referent. For an idea of how this "showing how" learning might have worked in practice, consider a prelinguistic version of Robbins Burling's experiences of pantomimic teaching while living among the Garos people of north-eastern India: 'I would occasionally ask someone to explain how to tie a knot or how to hold a knife when cutting bamboo... a man would reach for my tool and materials, demonstrate the manner in which the job should be done, and then hand them back' (Burling 2005, p. 184).
} 
increases in social learning due to mimetic communication, were apparently major fuels for success, as we will see in §4.1. And as will be discussed in $\S 4.2$, from about 2 Mya this period marks an evolutionary transition in vocal and neurological anatomy, paving the way for increases in vocal complexity, breath and motor control, and intentional response, presumably in an incremental, co-evolutionary tight-lock step (Morley 2013): increases underwriting the development of musicality.

\subsection{Oldowan cognition, lithic technology and hunting}

Oldowan lithics are basically rocks that have had flakes knapped off. The cores were probably used for smashing bones and cracking open nuts, as well as tenderising meat so that it is more easily chewable and digestible; the flakes were probably used for skinning and slicing carcasses.

An iconic "showing how" pedagogy, intentional or otherwise, would have enabled the imitative transmission and continuity of Oldowan lithic technology, and for my purposes I need not become concerned with the debate over the extent of non-passive teaching throughout the Oldowan. ${ }^{84}$ Evidence for stone tool-assisted foraging increases from 2 Mya, by which time tools were transported from their source as far as 12 kilometres, emphasising the energetic benefits from tool use despite the burden of carrying them (Antón et al. 2014), and providing material evidence of forward planning.

Successful large game procurement and carving is a highly cooperative enterprise, and although it may not have been a central feature of hominin foraging strategies until the Late Acheulean (Stiner 2002; Foley and Gamble 2009), there is plenty of evidence of this behaviour towards the Oldowan/Acheulean cusp, so the cognitive prerequisites for increased cooperation and forward planning were undoubtedly in place. Recent multidisciplinary studies of the 1.8 Mya Olduvai Gorge FLK Zinj butchering site (in Tanzania) strongly suggest that at least by the Oldowan/Acheulean cusp, our Late Oldowan ancestors were often enough highly proficient ambush hunters (Bunn and Pickering 2010), killing

\footnotetext{
${ }^{84}$ Although forward planning (i.e., plan-making) will appear in my narrative by the Oldowan/ Acheulean cusp, discussed in due course.
} 
many prime-aged medium-to-large mammals (100 to 350 kilograms). ${ }^{85}$ Their proficiency was such that we can assume that skilled hunting has earlier roots, deeper into the Oldowan, adding to the (presumably more opportunistic) mob-hunting/scavenging of earlier times. Of course this is not to say that big game was a major staple of Oldowan diets, but it was certainly present, and therefore so were the capacities for acquiring that game.

Thus the butchering site evinces systematic, targeted hunting, implying increases in intentional listening (if the auditory vigilance of other ambush predators like leopards are any guide), and coordinated planning, which necessitated neural reconstruction for a capacity to make and mimetically communicate plans. Weaponry used for the kill was shortrange, and 'without single-shot lethality. Such hunting demanded cooperation' (Sterelny 2016c, p. 36; see also Stiner 2002). It also required good theory of mind skills for teamwork (as systematic hunting is not merely mob-work), and probably the capacity to conceive of the task as a collective enterprise, agent-neutrally (Tomasello 2009; Tomasello and Carpenter 2007; Tomasello et al. 2005). The capacities of the extant great apes are very limited in these respects. Simple + systematic cognition was advancing strongly.

The site's fossil profile reveals that around $70 \%$ of the bovids were prime-aged. Such a rate is only seen in hominin prey-profiles. Lion or leopard prey-profiles for example contain many more old and weak animals and young juveniles. In my view, this undermines hypotheses that focus on passive hunting and offer a later (i.e., closer to the present) date for cognitive advances such as forward planning, increases in cooperation, and intentional communication. ${ }^{86}$ There is also recent evidence of ancient hominins procuring aquatic prey such as crocodiles (presumably quite a feat), turtles, and innumerable catfish at 1.95 Mya (Braun et al. 2010), which discredits the idea that Homo did not eat aquatic fauna till the Late Pleistocene (Broadhurst et al. 1998). So there is likely to be a range of subsistence strategies, depending on local resources and environment among other things.

\footnotetext{
${ }^{85}$ In the dry season many animals in the palaeo-Olduvai Basin were forced to travel to the only freshwater spring in the area, because the lake nearby was too saline to drink. The butchering site was just over 200 metres away from the spring, in a lightly wooded plain of shrubbery, trees and open swampland (Bunn and Gurtov 2013; Ashley et al. 2010).

${ }^{86}$ This is not to say that these cognitive advances were genetically entrenched and hence available to all descendant hominins from then on. The general picture, again, is an incremental one.
} 
Finally, coordinating hunting plausibly selects for the capacity to make, mimetically communicate, and remember short narratives, empowering the first (wordless) plans and hunting stories. Vocalisation for affect, attentive focus, and as an indexical aid (e.g., vocal mimicry of animals), would have been an effective addition to the manual acting-out of both plans and past activities, by the Oldowan/Acheulean cusp. ${ }^{87}$

\subsection{Oldowan musicality}

From 2 Mya the fossil record reveals neural and anatomic changes that intensified the incremental evolution of hominin vocal musicality (see Morley 2013 for review). These changes include loss of air sacs in the throat (possessed by Australopithecus afarensis-see Alemseged et al. 2006), a shift from the funnel-shaped chests of Australopithecus to barrel-shaped chests (permitting greater complexity of phonation-see Cross 2016), the enlargement of the hypoglossal nerve (for tongue control), and co-evolving vocal and auditory structures (neural and morphological-see Morley 2013), all occurring through the late period of this stage and throughout the next. And they would go on to include thoracic vertebrae nerve canal expansion, enhancing intentional control of breath and vocal musculature (MacLarnon and Hewitt 1999) down the line (see below). All this enabled improved voluntary control, regulation and basic forward planning of breath and mouth shape, and allowed an enhanced repertoire of producible vocal sounds and enhanced motor control (necessary for vocal mimicry, deliberate turn-taking, and so on), and underwriting more compound, continuous vocalisation than the earlier, discrete vocalisations of our Pliocene forebears.

The vertebral column expansion occurred throughout the following two stages of this narrative (that is, from ergaster/erectus to heidelbergensis) —and indeed, as far as it can be gleaned from the fossil record, by heidelbergensis at roughly 600 Kya our ancestors had roughly modern levels of vocal control and breadth of vocal sound repertoire. However, for these changes to be any use to vocalisation, neurological developments for top-down, voluntary control and basic forward-planning must have already taken place. Neurological

\footnotetext{
${ }^{87}$ Several aspects of the scenario envisioned, such as this, are obviously not essential to the framework, but are plausible add-ons, and should be treated as such. The picture does not turn on a specific view of the evolution of coordinating hunting communication, for instance; it is the mosaic nature of musicality that matters.
} 
developments such as these would have been bolstered through co-evolutionary feedback from the development of greater executive control. This co-evolutionary dynamic is likely to have progressed for a million years or so between early erectus/ergaster and heidelbergensis (Merker et al. 2015; Morley 2013).

Rudimentary forms of crooning/humming/chanting/laughing would have been an effective vocal grooming method as group size increased in the Oldowan, throughout the Acheulean, and beyond. According to the social brain hypothesis, increasing brain size (in particular, neocortex size) is a proxy for increasing group size (see Table 7.2), and larger group sizes call for methods of maintaining social bonds in a potentially one-to-many mode. That's because one-to-one fingertip grooming typical of apes (and primates generally) would be a much less time-efficient means for maintaining social bonds with enough members of the group, at least on its own. When group size increases, individuals in those groups face significant physiological costs resulting in social tensions; social grooming eases these tensions, and vocalisation is a way to involve several individuals at once. Dunbar originally proposed this scenario in the context of the evolution of protolanguage/gossip (see e.g., Dunbar 1996), but it better suits the evolution of vocal musicality (cf. Dunbar 2012), since these vocalisations are not likely to have been referential or semantic, but affective. Since 'social bonds are the product of a dual-process mechanism that involves bother a cognitive component (the social brain element) and an emotional element (derivative of social grooming and mediated by endorphins...)' (Gowlett et al. 2012, p. 699), the affective nature of these vocalisations should be central to this idea. The purpose of these vocalisations as a medium and amplifier for emotion does not imply that they resembled anything we might characterise as musical today (e.g., scales, modes, regular pulse), let alone any formal production (e.g., musical instruments). As Gowlett et al. note, it is 'the link between the movements, gestures, and feelings of the body' (p. 700) that is key.

Affective vocalisations could have been adult-to-infant proto-lullabies, small-groupfocused crooning, partnership or mating signals, or a mix of these and any others besides, and would have been an effective tool for social relating and producing changes in individual affect, especially as social group size grew larger and standard fingertip grooming 
became a much less time-efficient method for maintaining social relationships with enough members of the group. Following Dunbar:

For Anthropoid primates, and in particular Old World monkeys and apes, social grooming is the principal mechanism used for social bonding. Grooming is an intense activity in which one individual leafs through the fur of another, removing bits of vegetation, dead skin and other debris. It is very much a one-on-one activity, in which the groomer is often deeply concentrated on its task. Grooming lowers the heart rate in the groomee and reduces the frequency of signs of tension and stress (yawning, scratching, etc.)... to the point where the recipient of grooming can become so relaxed it actually falls asleep. Many species devote considerable proportions of their day to social grooming -in the limiting case, as much as one fifth of the waking day. Grooming shares many of the features of massage: it is physically stimulating and mildly painful, and thus triggers the release of endorphins... these psychopharmacological effects mediated by grooming seem to play a crucial role in the process of building the trust and reciprocity that form the basis of primate social relationships. (Dunbar 2008, p. 19.)

Group-based vocalising today releases endorphins in humans which encourage social bonding and group cohesion through eliciting emotion and affect. Endorphins released during group-based singing in humans today raise pain thresholds (Dunbar et al. 2012). And the connection is phylogenetically deep. Oxytocin (a neuropeptide that acts as a catalyst for the formation of trust between individuals) is released in humans during group singing, strengthening socio-psychological bonds, just as bonds are strengthened by betaendorphin release in one-on-one fingertip grooming in Old World primates (Keverne et al. 1989), building on those very ancient regions of our neuroanatomy (i.e., the hypothalamus 
as well as receptors scattered throughout the brain). ${ }^{88}$

I suggest that gelada baboons (Theropithecus gelada) may be a good vocalgrooming model (Richman 2000). As briefly discussed in chapter 6, geladas are primates that are organised into long-term harems (one male per harem, up to ten females, plus any dependent youngsters) within very large group-foraging bands of up to 200-300 individuals. The social structure of these large bands is multi-levelled and complex. To supplement fingertip social grooming, geladas have developed a form of vocal grooming.

Primatologists distinguish between two categories of vocalisations characteristic of adult geladas, contact calls and aggressive and defensive calls (Aich et al. 1990). Types and frequency of calls are correlated to an individual gelada's place in the social system. For instance, harem-leader male geladas use vocal calls to soothe tensions or mollify hostile exchanges between females co-situated in the harem, 'suggesting that maintaining crosssex bonds within a reproductive unit contributed to this instance of evolved vocal complexity' (Gustison et al. 2012, p. 1847). Here the vocalisation is playing a vocal grooming affective role (Richman 1987, 2000; Gustison et al. 2012).

Call-and-response vocal calls are utilised by grooming partners and in feeding, again playing a social grooming role; adult members of a harem call together in chorus (Dunbar 2014a). Moreover, much gelada group song can be seen more generally as an effort to maintain social cohesion through coordination. Bruce Richman notes:

The rhythm and melody of their singing are built out of successive parts that strongly contrast with each other in a digital, language-like way... [G]elada songs end with a very long ingressive sound with a distinctive slow rise in tone that serves as a discrete signal to other geladas that the song is ending. The rhythm of the singing is based on contrasts of kinds of beats

\footnotetext{
${ }^{88}$ Today, lullaby is widely used, cross-culturally, to relax and soothe, and that much affective music (for instance "sad" sounding music) triggers the release of hormones such as prolactin, causing a feeling of greater overall pleasure from listening to the music, despite any genuine feelings of sadness perceived too (Huron 2011). So music's effects seem to include those once dealt with through physical grooming (i.e., socio-psychological) in our lineage's deeper, primate-like past. And as well as emotional states, music today evokes vivid episodic memories, offering an "enriched context" for encoding contextual information (Ferreri et al. 2015; Belfi et al. 2015), again indicative of its socially-oriented past.
} 
and time placement. Other geladas have to identify these rhythmic contrasts in order to place their own beats synchronously. It is possible that the sound contrasts of the gelada vocal repertoire are the result of pressures towards discreteness imposed by the exigencies of choral singing. (Richman 1993, p. 722).

For all we know, Oldowan song may or may not have been much like this description at all. The bottom line here is that it is plausible that hominin musicality was put to use for a socio-psychological grooming effect, in some way or other, as we can see it clearly has in other socially complex primate groups. (Note this is not to say that human music is an adaptation for that function.) As noted, hominin vocal grooming-utilising the medium of sound, through the voice, to express and elicit affect/arousal—could have played multiple roles: group-based communicative affect, carer-to-infant bonding, and in the context of courtship (indeed, you might think that in social worlds such as these, individuals are constantly scrutinising each other for partnership making/unmaking and that vocalisations would be one unescapable factor). So again the crucial point is not the specific role played by vocalisations in particular contexts but that vocal musicality became a medium for social, emotive communication (as tool-making "showing how" communication presumably remained largely gestural). I envision simplistic vocal grooming as taking off in the Oldowan, and incrementally evolving in complexity and frequency in tandem with the co-evolution of brain size and group size throughout the Acheulean and onwards. I hypothesise that the vocal grooming scenario described here-or something much like itwould have intensified selection for coordination between voices, reinforcing our ancestors' increasing executive control and rehearsal of interval-based timing. It may also have selected for sensitivity to vocalisation "dialect" differences between groups, as social learning of vocalisation through ontogeny begins to kick in.

And vocalisation learning started earlier. In addition to the above, the neural and anatomical changes identified enabled babbling-a universal, deeply entrenched human infant behaviour - giving rise to better, earlier vocal control through vocal play-like soundproduction not observed in other primates, and thus finer executive control of voice/vocal 
musculature in adulthood (Merker 2012). This and all the above developments foreground the importance of vocal learning and an incrementally increasing phonological complexity. Vocal learning - the ability to mimic heard sounds-was another crucial aspect of Oldowan mimetic/iconic culture. Mimicry of animal calls are a crucial upgrade to a hunting band's toolkit-for a present-day example already mentioned in this thesis, some Native American tribes historically used a bleating calf song to round up a herd above a cliff face, which they would then force over. ${ }^{89}$ As I have already alluded, animal call mimicry would also be an effective addition to gestural/pantomimic acting-out of the first hunting plans and pedagogical or status-securing hunting stories, which in turn rehearse standard theory of mind capabilities. I suspect this would have had a major impact on the evolution of storytelling.

\section{Stage Three: the Acheulean}

Hominin encephalisation and group size increase continued throughout the Acheulean (Table 7.2). For the next million years or so, our ancestors slowly developed increasingly ritualised vocal musicality as well as mimetic and indexical gestural communication, as I shall argue. Between 1.5 and 2 Mya, our ancestors ostensibly gained 'the neural foundations for language (Broca's area) and complex behaviours supported by an expanded frontal lobe' (Barham 2013, p. 67), as is indicated by endocranial evidence of hominin specimens (e.g., KNM-ER 1470, dated to 1.88 Mya; KNM-ER 3733, dated to 1.78 mya; KNM-WT 15000, dated to 1.5 Mya—see Shipton and Nielsen 2015).

Our Acheulean-era ancestors migrated throughout the Old World, developed increasingly systematic tracking skills for animal hunting ${ }^{90}$, and cultivated an increasingly communicative culture. Gesture and mime, in turn, accelerated social learning and imitation, as evidenced by the Acheulean toolkit, which empowered demographic

\footnotetext{
${ }^{89}$ Furthermore: 'Eskimos imitate cries of geese, swans, and walruses to bring these animals within shooting range. Australian aborigines mimic a hawk alarm call to get a running lizard to freeze so that it becomes an easier target' (Orians 2014, p. 128).

${ }^{90}$ As excellent pattern-recognisers, it appears that modern humans are very good at recalling animal tracks-not as good as they are at recalling kitchen utensils (i.e., tools), but better than recalling other categories, such as trees, seashells and military vehicles_even though most modern humans have little if any tracking experience (see Sharps et al. 2002).
} 
expansion and the wide-ranging occupation of new environments during this time, in the face of climatic variability.

Stephen Shennan's simulations have suggested that demographic expansion and adaptive cultural innovation tend to go hand in hand. That is, cultural innovations tend to be more beneficial in larger rather than smaller populations.

When cultural innovation processes take place and the results are passed on by a combination of vertical and oblique transmission, larger populations have a very major advantage over smaller ones. Quite simply, members of larger populations are on average both biologically fitter and more attractive as models for imitation, by virtue of the fact that the deleterious sampling effects present in small populations decline as population sizes increase. When populations are small, innovations which are less beneficial reproductively and less attractive to imitate are more likely to be maintained within them. (Shennan 2001, p. 12).

Demographic expansion also underscores how important systematic trackways reading would have become for gaining folk-biological information facilitating the procurement of animal meat, hides and other resources in new environments (see ShawWilliams 2014).

Systematic trackways reading is an intentional activity-a planned strategy of hunting for a target. Individuals have to have a target agent or species in mind before finding the footprints, to decipher much less obvious signs of that target's progress through the environment, and to imagine where they are going when their footprints are temporarily not registered by a substrate (in rocky beaches and outcrops, for example). Systematic tracking (i.e., intentional, dedicated examination of all terrains not only for footprints but also dislodged stones and leaf litter, brushed forward grasses, broken foliage, torn moss, cracked twigs, scratched rocks, blood spots, caught hair and fur) of other large mammals likely emerged in the face of Oldowan environmental mayhem. As we saw at the Oldowan/Acheulean cusp, by 1.8 Mya (and probably much earlier, given the 
hunting proficiency evident), our ancestors procured larger animals for sustenance and in doing so, their presumably more opportunistic hunting and simple tracking gave way to systematic, planned tracking and hunting. The Acheulean stage saw the development of these skills.

Systematic trackways reading is a skill that can always be improved. It requires a good working memory for ordinary animal behaviours and an integration of natural history with local geography. Theory of mind, episodic memory and inductive reasoning (that is, we are now beginning to see the beginnings of simple + systematic + speculative cognition) were rehearsed and further elaborated through systematic tracking. Not only is systematic tracking a useful hunting strategy, it aids predator avoidance, water gathering and foraging (since specific animal tracks can lead to edible resources and waterholes), and navigating rough terrain.

The Acheulean saw the development of more complex lithics than the Oldowan (that is, cleavers and bilateral handaxes). Archaeologists distinguish the cruder Mode 1 (Oldowan) stone tools from Mode 2 (Acheulean) tools. The established construction and use of stone tools by adults in the Oldowan created an environment in which the juveniles could acquire tool-based skills earlier on through play and exploration, leading to increases in tool-based competency. Acheulean handaxes, first appearing from around 1.8 Mya (see Figure 7.2), are evidence of this very increase in competency-they are knapped flakes shaped to a somewhat standardised multi-functional profile: a kind of teardrop shape. These superior tools enabled better handling and easier access to areas of animal carcasses otherwise trickier to get at (including that of carcasses that otherwise would have been ignored if other predators have had a good go at it first); that is, enabling access to marrow in bones and jaws, tongues, brains in skulls. For example, consider the well-butchered elephant bones at Barogali, dating to 1.6-1.3 Mya (Berthelet and Chavaillon 2001). Consequently, kills/scavegened carcasses became much more resource-rich and energetically cost-effective. 


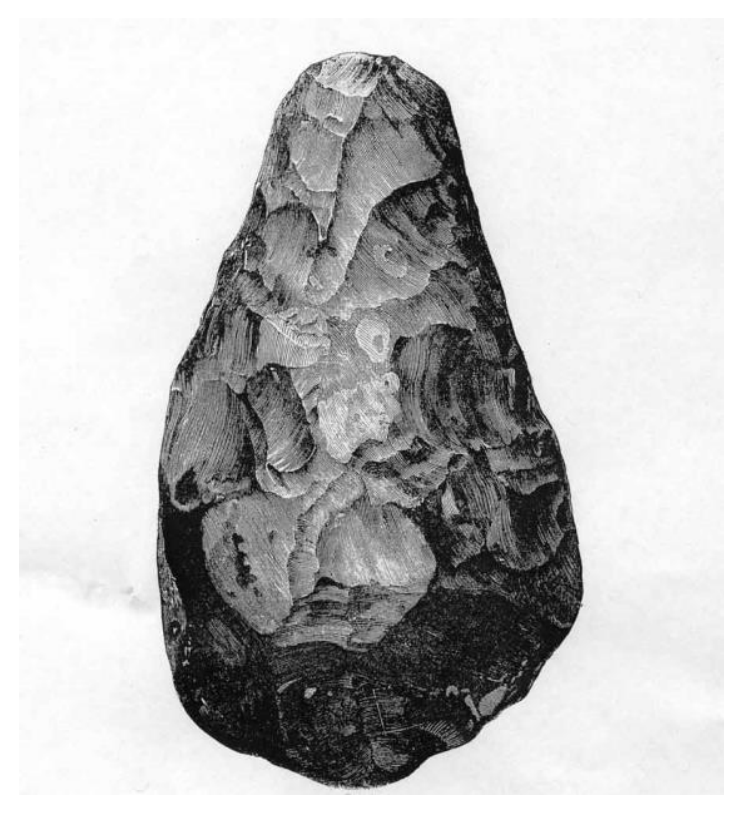

Figure 7.2: Acheulean handaxe ${ }^{91}$

Acheulean tools, moreover, suggest to me more complex methods of social learning than the "showing how" pedagogy of the Oldowan. Nonetheless some theorists disagree with this. Tomlinson (2015) argues that Acheulean lithics even as late as 1 Mya evince merely unplanned series of physical actions, transmitted only by way of simple mimetic copying and individual trial and error processes. I am sceptical (Killin 2016b). Consider the production method of the stone cleavers of Isampur Quarry, dated to 1.21 Mya:

... the entire manufacturing sequence from clast procurement to finished tool is preserved with spatial integrity for handaxes and cleavers... Handaxes were made by reducing thin slabs of limestone to leave the teardrop handaxe shape parallel to the bedding plane of the slab. Cleavers, by contrast, were made by setting up platforms on thick slabs of limestone from which large flakes could be struck obliquely to the bedding plane, which were then retouched into the cleaver shape... The cleaver

${ }^{91}$ Figure adapted from the Victoria County History of Kent Volume 1, 1912, and therefore now in the public domain. Source= https://en.wikipedia.org/wiki/File:Acheuleanhandaxes.jpg. Accessed 20/08/2016. 
manufacturing sequence involves several hierarchically organised stages, and to a novice it would not be obvious how some of the earlier stages relate to the finished cleaver... Such preparatory steps include removing thick flakes from the slab perimeter to set up suitable platforms from which to strike the large flakes that will then be made into cleavers. To understand why this platform setup step is necessary requires some experience of knapping to know the angles and surfaces that are good for striking large flakes. (Shipton and Nielsen 2015, p. 336).

In my view, it is near inconceivable that such a production method could be the inevitable outcome of unplanned patterns of physical actions (cf. Tomlinson 2015). If that does not convince, consider the constraints imposed by the large slab cores $(\sim 65 \mathrm{~kg})$ of Isampur Quarry from which flakes were struck:

Experimental evidence shows that stabilising the core could be achieved in one of two ways: either one person could lift the core while another put dirt underneath to cushion it and prop it up, or the second person could hold the core directly while the first person strikes it... Either way, producing large flake blanks from these giant cores was apparently a two-person job. (Shipton and Nielsen 2015, p. 339).

This does not look to me like the unplanned work of tool-making zombies. So in my view, Acheulean lithics from 1.8 Mya onwards reveals salient cognitive advances (see also Hiscock 2014; Jeffares 2010; Shipton 2013):

(1) The emergence/concretisation of shared attention, overimitation, and shared intentionality (Shipton and Nielsen 2015). Shared intentionality plausibly rehearses higher levels of theory of mind (at the very least it involves recognition of another's intention), selecting for the kind of neural reorganisation for speculative cognition to emerge and incrementally evolve. 
(2) Increases in episodic memory (recalling the "feeling" of the weight, angle, and shape of past successful knapping gestures, making intentional repetition possible), forward planning, and possibly rudimentary mental time-travel (again, the beginnings of speculative cognition). During the early stage of the Acheulean (i.e., 1.8 Mya) tools were transported from their sources for up to 13 $\mathrm{km}$. This increased to a whopping $100 \mathrm{~km}$ from source by the mid-Acheulean at 1.2 Mya (Petraglia et al. 2005; Marwick 2003). In contrast, chimpanzees might select a nearby rock and use it as a hammer, but they do not take the rock with them for much more than a few minutes down the line. Moreover, Marwick argues that the extensive increase in transport of Acheulean lithics indicates the presence of communication, 'the ability to pool information collected by individuals through face-to-face negotiation' (Marwick 2003, p. 71). If that's right, simple mime and gestural communication (with adjunct vocalisations, for instance to direct attention) would have been an effective means of communication for such Acheulean meetings: it need not have been symbolic, lexically-rich, syntax-laden, or even "about" the elsewhere-and-elsewhen. See point 6, below.

(3) Mental entertainment of model templates (Mithen 2002). These mental visions of the end-product would have been crude at first, presumably - and there is much variation in tool shape/symmetry in the archaeological record (e.g., Vaughan 2001; McNabb et al. 2004; Petraglia and Shipton 2008)—though increasing in fidelity over time.

(4) Increased attentive focus and good impulse control. When tools are being made for future rather than opportunistic use, their benefits are not in the here-and-now, so completing their manufacture (including sourcing raw material, testing it for suitability, as well as knapping the artefacts themselves) requires some level of commitment to the tasks at hand in the face of momentary distraction. So future-oriented complex lithics (for activities in the “elsewhere and elsewhen") requires careful attention and impulse control on the part of the tool maker (Jeffares 2010). 
(5) Greater manual dexterity and top-down fine-grained motor control as the hominin hand, brain and repertoire of tools co-evolve (see e.g., Marzke 2013).

(6) Upgrades in social learning and communication (see Hiscock 2014 for a detailed review of stone-knapping and its cognitive demands). In my view, gestural/manual communication (pointing gestures, manual mimetic expressions, and so on, building upon the basic capabilities of chimpanzees), alongside vocal utterances for emphasis/attention, would have provided an effective means to aid transmission of lithic traditions through subsequent generations, and to facilitate exchange and negotiation (as per Marwick 2003). Upgrades in gestural communication would also have enabled effective silent coordination of group hunting and simple-to-systematic animal-trackways following, and would have co-evolved with upgrades in manual dexterity and top-down control (see point 5, above).

Moreover, in my view, Acheulean lithics underscores the importance of intentional listening, rehearsing and developing greater intentional listening and response to sound, which underscores an important upgrade in hominin musicality. Sound is an effective diagnostic tool for testing the suitability of raw material for knapping (indicating for example properties of the stone such as internal density). And in knapping itself, as Morley points out:

Anybody who has carried out flint knapping will know that it requires a great awareness of the sound that the stone produces as it is struck. A skilled knapper has to be aware of the subtlest variations in the sound produced by the stone being struck, as this provides important clues as to where and how hard to strike the stone next. (Morley 2013, p. 120). 
And as Larsson points out:

Tool use, and its associated sound, has accompanied humans throughout millions of years. Hammering is likely to have been a commonly heard sound... Slicing flesh from bones, cutting vegetation, cracking nuts, and the use of grinding utensils in food processing represent tool uses in human prehistory that were potential sources of TUS [tool-use sound]. (Larsson 2015, p. 997).

I hypothesise that the intentional awareness of sound in knapping and tool use may have led to the experimental use of stones and flakes for their musical qualities-a kind of lithic sound-based play_-recreation in an environment in which stone tools are produced and utilised, the sounds of tool production and utilisation are heard, and so on. ${ }^{92}$ Tool-makers themselves would have been automatically exposed to a lithic soundscape as a side-effect of knapping and anyone else listening in may too have become intrigued, for instance alleviating boredom (a big cost associated with growing bigger brains!). And the longer juvenile period resulting from changes in life-history stages during the Pleistocene (see e.g., Barham 2013; Petraglia et al. 2005) extended the time that individuals were engaged in playful activities: here I am envisioning juveniles, who grew up in a developmental environment wherein lithic sound was recurrent, engaging in sound play through fooling around with discarded rock or idle/unoccupied anvils-building upon and extending Oldowan vocal/sound play capacities. Rehearsal of sound play ${ }^{93}$, which perhaps also included vocal mimicry of lithic sound and playful synchronisation of sound patterns (that is, musical entrainment), plausibly provided a key developmental step from intervalto beat-based timing, gesturing towards further creative appropriation of natural resources for sound-play purposes. And imitation games too may have figured here in the

\footnotetext{
${ }^{22}$ Other theorists have hypothesised that the intentional awareness of sound in knapping led to the use of stones and flakes for their musical qualities very early on (Cross et al. 2002; Zubrow et al. 2001). Once a sophisticated tool-making tradition established, there would have been a great deal of natural experience of, and experimentation with, percussive sounds.

93 There is some suggestive support for this in modern human ontogeny: 'Replication, sound play, and noise experimentation all seem to be important aspects of a child's prelinguistic development' (Larsson 2015, pp. 999-1000).
} 
development of beat-based timing: consider a youngster playfully imitating an adult knapping rock, synchronising actions by sight and thus subsequently producing synchronised sounds as a side-effect of that visual imitation. Later playful, creative and speculative appropriations of natural resources for more sophisticated musical purposes would continue in later stages in this bio-cultural narrative as it continues to do so today. ${ }^{94}$

Whether we already have material evidence of sound play is yet to be determined. Goren-Inbar et al. (2015), for example, describe thin basalt anvils newly discovered, and the possible activities associated with them (e.g., nut-cracking), at Gesher Benot Ya'aqov (Late Acheulean: $790 \mathrm{Kya}$ ) (although note that hominin use of anvils dates back to the Oldowan: see Leaky 1971). Use-wear/experimental analysis of artefacts such as these might reveal that playful percussion is likely to be a cause of some damage markings. ${ }^{95}$ So testing these ideas is one key direction for future research. Spontaneous sound play with discarded human artefacts found by young wild chimpanzees has been observed (see Matsusaka 2012). And percussive-material play seems to be a developmental step, at least sometimes, towards the emulative social learning of percussive nut-cracking behaviour in some chimpanzee populations (see Whiten 2015). These data points are suggestive and further research might shed some light here. Some great apes have been taught to knap stone flint tools by humans (e.g., Roffman et al. 2012): it is tempting to wonder how and if juvenile lithic sound play resembling the above might emerge within "knapping ape" generations to come.

The role of play (sound-based or otherwise) should not be dismissed as frivolous from an evolutionary perspective (see Bateson 2017; Bateson and Martin 2013). Play prompts plasticity; play improves one's motor skills, one's awareness of environmental contingencies (consider again diagnosing stone for tool production through sound), and so

\footnotetext{
${ }^{94}$ For example, ancient, intentional striking on stalactites and stalagmites has been evidenced in the booming, echoing caves of the Réseau Clastres in the French Pyrenees (Lewis-Williams 2002; Montelle 2004). Indeed there are many simple ways to be musically creative with natural resources that would not surface in the material record too.

${ }^{95}$ These researchers do not seem to consider this as a possibility, focussing on the presumably primary function of anvils in food processing: 'At present, we cannot associate any particular use to the damage markings on the anvils, but they may have included nut cracking, possibly nut popping, bone fragmentation for the extraction of marrow, which is very common at the site, cracking open the shells of crabs and bivalves, fruit smashing (as practiced by chimpanzees) and possibly the preparation of other plant foodstuffs such as underground storage organs' (Goren-Inbar et al. 2015, pp. 7-8).
} 
on, having an effect on the evolution of these abilities in the population, expanding the search-space through which evolutionary novelties might be found.

There was another important Acheulean social development triggered by a major change in morphology. Birthing difficulty caused by encephalisation (given hominin bipedal morphology) selects for earlier birthing and thus longer childhood, resulting in longer care periods, food dependence and commitment from parents (Antón and Snodgrass 2012). Energy saved from slowing the growth of human juveniles into adulthood (i.e., spreading growth across a longer period) can be spent on growing bigger brains, and the effects of prolonged juvenility are counterbalanced by increased cooperative allocare.

The shift to bipedalism by 4 Mya saw the rounding out of our ancestors' ilial pelvic bone and the closing-in of the ischium, narrowing the birth canal through which a baby's head (then much smaller, so it was much less of a problem) must pass. Yet as our evolving, bigger brains required bigger craniums to hold them, eventually this presented a birthing problem in our Oldowan/Acheulean maternal ancestors, known as the obstetrical dilemma. Present-day female non-human primates give birth relatively quickly and without much difficulty. Not so for modern women, let alone our Acheulean great-grandmothers, who compromised by giving birth to underdeveloped infants. The gestation periods of all other mammals are set by ratios involving the species' brain size. Humans, if not for our bipedalism, should have a gargantuan gestation period of 21 months (Dunbar 2004). This early-birthing phenomenon was well in place by the next stage of the narrative, the Late Acheulean, by the time of heidelbergensis (Dunbar 2014a), so it is during the Acheulean period that it (presumably incrementally) emerged.

Various researchers have stressed that a crucial upshot of hominin early birthing is increased sociality in reproductive domains (Hrdy 2009; Hawkes 2003; Burkart et al. 2009). For one, given our bipedal morphology, humans usually require birthing assistance. A birthing mother cannot easily guide her baby through her birth canal. Non-human primates, on the other hand, seek seclusion; no assistance is needed. More crucially for my narrative, 
though, alloparents become crucial; it can take a tribe to raise a human child. ${ }^{96}$ I suggest that an increase in allocare saw an increase in vocal grooming (lullaby/crooning) as parents and alloparents employed their vocal musicality in efforts to excite, calm, or produce other alterations in the affect and emotion of their underdeveloped young (Falk 2004)—much as today's ubiquitous and universal tendency for carers to engage in infant-directed speech or so-called “motherese” does likewise (e.g., Bryant and Barrett 2007; Grieser and Kuhl 1988). Soft (human) vocal music reportedly has a calming effect on captive chimpanzees (Howell et al. 2002), suggesting an ancient, pre-existing bias in the Pan-Homo last common ancestor.

As Gerhardt (2004) explains, the orbitofrontal cortex manipulates an individual's affect (and ultimately behaviour) by controlling her emotions-a part of the brain that is not fully developed until well after infancy-implying that it is shaped by the input of her carers and other features of her developmental environment. Several evolutionary theorists stress the importance of infant-directed speech/vocal musicality in development (e.g., Mithen 2005; Gowlett et al. 2012). Children brought up in a developmental environment in which musicality is expressed for affect are more likely to continue to express musicality themselves through play, after infancy. In turn, they themselves employ musicality for its affective power as parents or alloparents. This is niche construction in action: musicality increases and evolves through feedback loops and social innovation, driving neurological advances that inter alia enable greater expression of musicality.

Reviewing the fossil record, Barham (2013) puts the initial evolution of a childhood stage (between infancy and juvenility) by 2.3 Mya, and an extended childhood stage and later juvenile stage from 1.9 Mya. A further-extended childhood stage and an even later juvenile stage are placed at the cusp of this era and the next (Late Acheulean) at 900 Kya. The emergence of an adolescent stage (between juvenility and adulthood) is placed at 500 Kya, which, by 160 Kya, has even further expanded (i.e., teenagehood). (See Figure 7.3.)

\footnotetext{
${ }^{96}$ Here I echo Antón and Snodgrass, who suggest the following. 'In cooperative breeders allocare (including paternal care) allows the mother to channel resources to her own somatic maintenance and reproduction; thus, allocare should generally be favoured evolutionarily when the risk to the offspring is not too high... The well-developed system of cooperation in humans plays a critical role in supporting the high costs of encephalisation that must be paid during pregnancy and lactation... enabling early weaning, relatively low extrinsic mortality, extended subadult dependence, and high fertility... Thus, cooperative breeding was almost certainly a critical contributor to brain size increase in the Homo lineage' (Antón and Snodgrass 2012, p. 5489).
} 


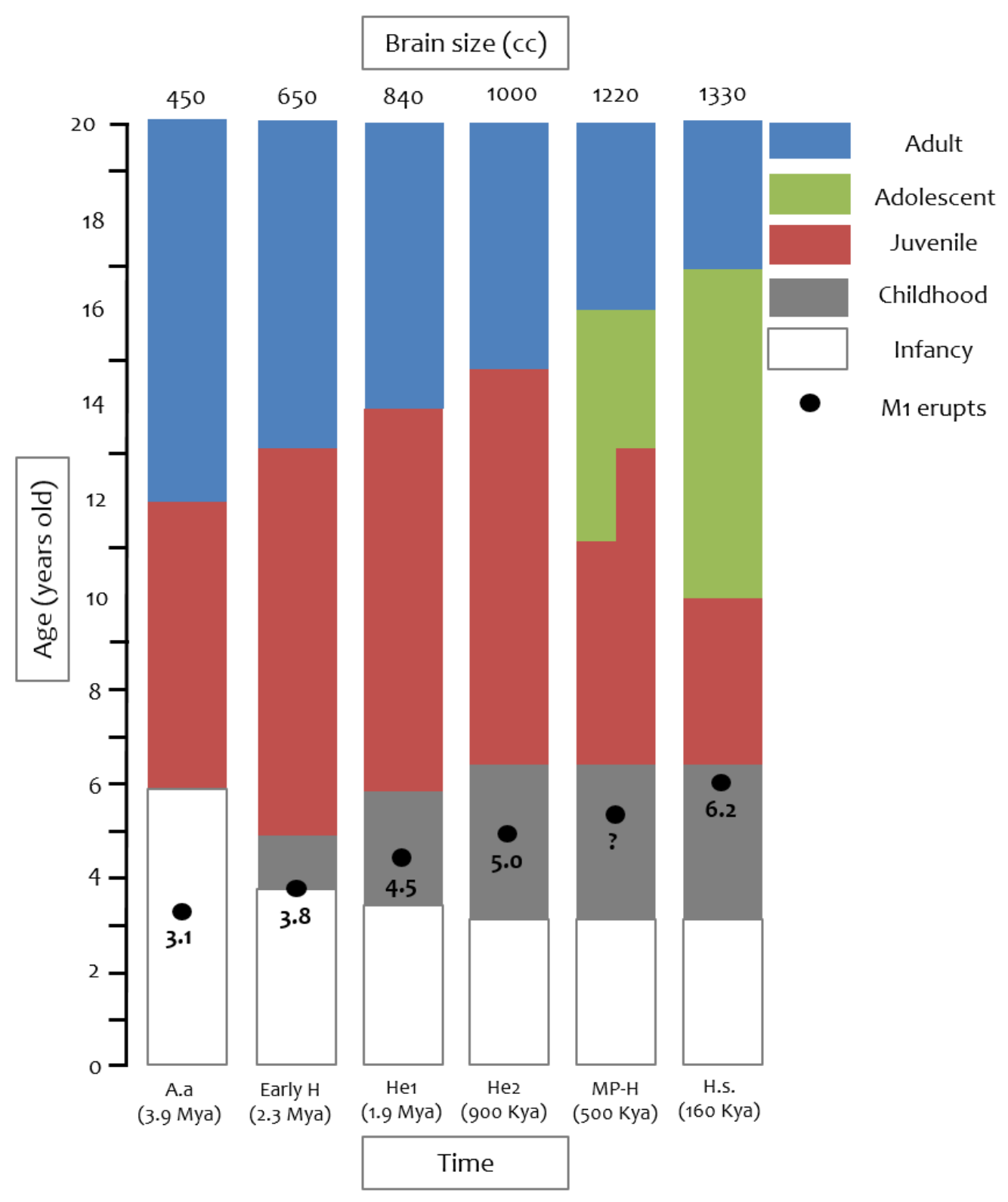

Figure 7.3: The evolution of human life-history stages ${ }^{97}$

\begin{abstract}
97 Recreated figure, based on Barham (2013) Figure 3.3 and data. 'The evolution of human life-history stages showing the estimated time of the distinctive pattern of an adolescent phase before reaching physical maturity. The age of eruption of the first molar (M1) and mean adult brain size are used as proxies for reconstructing the sequence. A.a = Australopithecus afarensis; Early $\mathrm{H}=$ early Homo; He1 = early Homo erectus; $\mathrm{He} 2$ = later Homo erectus and includes Homo antecessor (TD6) as a possible early outlier marking the potential for an adolescent stage 900,000 years ago; MP-H = Middle Pleistocene Homo; and H.s. = early Homo sapiens' (Barham 2013, p. 102).
\end{abstract}


So longer childhood and extended parental care allowed more pre-adolescent play (and practical helping behaviours, see Kramer 2011). And, indeed, enlarged social groups created the environment in which longer periods of development (life-history stages) could be selected for. Additional play time allowed for the exercise of higher-order, more complex intentionality/“theory of mind" capacities (Tomasello et al. 2005), imitation of adult roles and eliciting feelings/emotions and thoughts (Barham 2013), as well as 'more time for cognitive development, including the development of ecological skills such as in foraging as well as the refinement of social behaviors' (Antón and Snodgrass 2012, p. S488). During their long juvenile period, children develop increasingly complex intentional competencies. This would have led to more complex intentional social expressions in the adult sphere, one result of which I hypothesise is increasing musicality, especially the emergence of novel modes of playful expression of musicality.

In sum: the Acheulean stage saw the further elaboration of the ecological and sociocognitive niches of our Oldowan ancestors, incrementally through better trackways reading, stone tool production, and creative play which included musicality. This foregrounds our Acheulean ancestors' emerging intentional, systematic or playful musicality from the simple/opportunistic (and more purposeful) musicality of the Oldowan, described earlier. Our Acheulean ancestors' increased manual dexterity for tool production and gestural communication seamlessly co-evolved. Increased social learning gave rise to more complex skill specialisation characteristic of the social groups of the next stage of this model of Homo evolution, in which we see proto-musical behaviour (i.e., behaviour more distinctively "musical" than what I have been discussing so far) emerge from our deep "musicality"-mosiac. Thus the narrative continues in the next chapter, from the Late Acheulean onwards.

\section{Summary}

My co-evolutionary, socio-cognitive niche model of hominin evolution sees the incremental expansion of rudimentary core musicality, rising dynamically via the socio-cognitive increases afforded at least initially by the ecological resetting of our mobile, bipedal foraging Pliocene ancestors. Key ideas are as follows. Over roughly 2 million years, vocal musicality took on a social effect for expressing emotion and affect (potentially 
manipulative, suggesting increases in theory of mind). Vocal babbling, vocal mimicry and musical lithic play developed, intentional listening was rehearsed in multiple domains, and increased intentional vocal musicality and vocal grooming selected for subsequent vocalanatomic co-evolution (e.g., auditory anatomy and neural structures geared towards picking out and processing the vocalisations of conspecifics—see Morley 2013). Increases in interval-based timing incremendtally led to beat-based timing. Longer childhoods allowed more pre-adolescent play time and exercise of practical helping behaviours (Kramer 2011), higher-order intentionality/theory of mind (Tomasello et al. 2005), imitation of adult roles and eliciting feelings/emotions and thoughts (Barham 2013), and development of ecological skills such as in foraging (Antón and Snodgrass 2012), like better ecological knowledge (e.g., identifying edible plants and animal trackways), and so on. All this paved the way for increases in intentional social expression in the adult sphere-with one result being the incremental emergence of more complex musicality. 


\section{Chapter 8: Evolution of music}

In the previous chapter I argued for a socio-cognitive niche construction picture of the evolution of Oldowan/Acheulean musicality, connecting dynamic developments in hominin musicality to what I take to be the most persuasive interpretations of the evidence at hand, in the general context of understanding hominin evolution, and hypothesising additional factors that I take to be both consistent and independently plausible. This chapter picks up the narrative where the previous one left off, in the Late Acheulean (from roughly 800 Kya), and continues to detail my narrative of the evolution of musicality and the emergence of music. I argue that Heidelbergensians evolved something worthy of the (admittedly vague) designation "proto-musical" (that is, beginning to resemble something that we would consider more distinctively musical, in the vernacular sense, than that discussed in the previous chapter). From there, full-fledged music would evolve through the long period of behavioural modernity (from 250 Kya onwards), global dispersal from Africa (60-100 Kya), and throughout the Holocene (from $10 \mathrm{Kya}$ ), giving rise to the many musics of the world's cultures today.

\section{Stage Four: the Late Acheulean}

During the Late Acheulean, a final, momentous increase in encephalisation occurred (Antón et al. 2014), which, in tandem with the developments in musicality already underway, enabled in due course the utilisation of vocal musicality for the beginnings of spoken protolanguage from the gestural mode of "showing how" communication (and so on, discussed in the previous chapter). The previous stage, the Acheulean, was an era of vocal/anatomic co-evolution, selecting for the ontogenetically lowering larynges of humans as our species became even more verbally communicative (Fitch 2000; Morley 2013). Lower larynges extend the resonance chamber formed by the throat and mouth, which greatly increases vocal ranges and the sounds producible; the first evidence for this occurring is in Homo ergaster/erectus (Morley 2013).

At least by heidelbergensis (roughly 600-800 Kya; the ancestor of both modern sapiens and Neanderthals), the fossil evidence suggests that our ancestors were more or 
less morphologically capable of the modern human vocal repertoire and had evolved near-modern top-down vocal control (see e.g., Morley 2013; Dunbar 2014a). Whether Neanderthals (appearing from $400 \mathrm{Kya}$ ) had anything like archaic sapiens' linguistic-vocal capacities is hotly debated (Johansson 2015) however it is clear that both hominin species would have been capable of near-modern vocal musicality (Mithen 2005) $\cdot{ }^{98}$ However, no uncontested evidence of Neanderthal musical technology (e.g., flutes) has been discovered. ${ }^{99}$ Nonetheless, Mithen argues that Neanderthals may have been more musical than the evidence suggests.

In any event, throughout the Late Acheulean and onwards, I argue that musicality was not merely present as a part of ancient hominin life, but was utilised by other emerging cultural activities and behaviours (as suggested by Gowlett et al. 2012). I suggest that protomusic in some form became a crucial ingredient in the cultural niche construction of this era, with far reaching consequences.

In chapter 7 I characterised earlier forms of intentional informational communication as primarily gestural. Obviously somehow the switch was made to a largely verbal form of language. I think our Acheulean mimetic (iconic) and indexical gestural lexicon, and "showing how" pedagogy, was combined with vocal utterance for attention and affect, vocal mimicry, and prosodic humming/singing/chanting/laughing_-possibly gossip-like, as per Dunbar's suggestion—evolving eventually into the multi-modal verbalgestural languages of today as sounds and gestures took on arbitrary/conventional meanings. Robbins Burling (2007) for example argues that proto-musical or music-like vocalisation had something to do with the transition from gestural communication to spoken words. I agree: vocal musicality provided the medium for the switch. ${ }^{100}$

\footnotetext{
${ }_{98}^{8}$ Both Neanderthals and sapiens have the FOXP2 gene mutation for speech; both have wide canals for thorax-bound nerves (associated with intentional breath control); both have modern hyoidbones. However, Neanderthals lacked the larger sapiens pharynx. As far as there is any consensus, however, most researchers agree Neanderthaler vocal range was restricted in comparison to that of archaic sapiens (see e.g., Renfrew and Bahn 2012).

${ }_{99}$ The Divje Babe I "Neanderthal flute" (a femur bone of a juvenile cave bear, dated to 60 kya) turns out not to be a flute at all (Diedrich 2015). Evidence suggests that this flute-lookalike was simply created by a cave bear puncturing another cave bear's femur (see also Morley 2013, pp. 38-41). ${ }^{100}$ Liz Irvine (personal communication) argues that once group size of simultaneous communicators reaches greater than three or four individuals, various considerations (line of sight, re-focus and control, and so on) favour a shift from a largely visual to a largely auditory mode. (See also Sterelny 2016a, b.)
} 
Silberbauer (1981), for one, provides some tantalising inductive ethnographic evidence, given his observations concerning the fluid relationship between speech and chant/song in central Kalahari Desert tribes during daily group conversations and story-telling. I emphasise the context of fire culture in facilitating this switch (see also Wiessner 2014; Dunbar 2014b).

The Late Acheulean also saw major climatic chaos-longer and more intense largescale glacial cycles, more extreme hot/cold temperatures, greater seasonality/rainfall fluctuations-taking place from $900 \mathrm{Kya}$ and becoming more pronounced from $430 \mathrm{Kya}$ (Barham 2013). One effect of all this is 'greater unpredictability in the availability of basic resources' (Barham 2013, p. 11). According to Shaw-Williams (2014), this was combatted by an upgrade in trackways reading abilities: during this stage speculative trackways reading began to emerge from systematic tracking, requiring imaginings and successful conjectures of the target's present location to short-cut the tracking process, utilising the full cognitive repertoire of our Late Acheulean ancestors (theory of mind, mental time-travel, forward planning, and so on: increasingly advanced simple + systematic + speculative cognition).

\subsection{Late Acheulean technology}

Encephalisation, socio-cognitive upgrades, and climatic stress enabled hunting techniques to advance during this time (Barham 2013), including the use and production of complex, elongated javelin-style spears with flame-hardened tips, appearing from around $400 \mathrm{Kya}$ (Thieme 1997) —resembling modern-day sporting javelins. Their use implies a basic understanding of folk physics (see Zilhão 2007). If an animal target is on the move, the narrative self-projecting mind of an experienced hunter can predict the animal's future position and-with some crude understanding of folk physics—-be ready to throw, with the right angle and strength, when that animal is nearing the target position. Although this predictive skill could possibly be learned associatively through long practice, I suspect the complexity of the task hints at advances in episodic cognition and mental time-travel. Recalling past successes when learning - the feeling of the grip, the angle and pressure of the throw, the balance of one's stance, and so on-lends a higher probability to a javelin throw meeting its thrower's target. Such behaviours are imitating a past self. And it is 
plausible that these advances in the production and use of hunting technology at this time point to a division of labour/specialisation of skills.

From around 500 Kya, two further innovations appear: finely-crafted, symmetrical yet overwrought and unused stone handaxes that, famously, seem to surpass ordinary utility (Kohn and Mithen 1999; Mithen 2005), and, second, hafted tools (Barham 2013). Consider first the overwrought handaxes. These are evidence of impulse control and careful attention. Moreover, they further elaborate the intentional listening that supports better knapping, they suggest skill specialisation, and in my view, they reveal a proto-aesthetic sensibility (an over-emphasised sensitivity to visual symmetry, and so on). One common interpretation of the role of these axes is as honest, because they are costly, sexual signals (Kohn and Mithen 1999). This is controversial, however (see Nowell and Chang 2009). An alternative is that they are pedagogical props: oversized examples for teaching/learning purposes. (Or that they are social signals more generally.) Either way, they represent a high point of several million years of stone artefact production, ever-increasing social learning culture, and perhaps the beginnings of the ability to abstract, since these axes-obviously the kind of artefacts that ought to have a clear utilitarian functionality—instead embody some alternative role and were never put to their normal utilitarian role (whether that is as honest signals, or as large, clear, excellent exemplars, and so on) and presumably were pleasing to behold.

Second, until $500 \mathrm{Kya}$, all known tools were all made of a single source material and were all hand held. ${ }^{101}$ After $500 \mathrm{Kya}$, handles/shafts were increasingly added to stone tools; these are tools produced not merely by reducing and shaping raw material, but by adding distinct components together. Barham (2013) calls these "hafted" tools. No other animals do this; even chimpanzee tools (such as termite wands) are simple, single-source items. As Barham explains, hafted tools are further evidence of increases in forward planning, knowledge for construction, good working memory, raw material manipulation, social learning and teaching (implying high levels of intentionality/theory of mind), and a rich toolkit—presumably a prerequisite for later technological innovation of musical instruments, after possibly co-opting tools for musical effect (see later on).

${ }^{101}$ Of course, soft material technologies like nets or containers, that would not preserve, may well be different. 


\subsection{Late Acheulean fire culture}

There is good evidence of control of fire from 790 Kya (Goren-Inbar et al. 2004). Wrangham (2009) and Wrangham and Carmody (2010) place cooking/fire control even earlier with the Habilene/Erectine transition; others place it slightly later, around 1.6 Mya (see Roebroeks and Villa 2011; Attawell et al. 2015 for review), however I suspect that that early stage is, at best, one of only partial control of fire, and opportunistic or sporadic use. Indeed, fire usage has a patchy archaeological signature, and it cannot be assumed that once it was harnessed, it remained a permanent feature of our ancestors' lifeways. As Roebroeks and Villa note, hearths and other evidence of fire control became much more archaeologically visible from around $400 \mathrm{Kya}$, from which point there is widespread, continual evidence of skilled control of fire. Nevertheless my story is an incremental one. Hearths, after all, are not a full proxy for hominin fire use. So I take the view that fire was harnessed opportunistically at first (in the Acheulean stage) ${ }^{102}$ and, over time, became habitual (in the Late Acheulean stage), by 400 Kya.

Recent research by Richard Wrangham and various collaborators has emphasised the significance of fire and cooking in hominin evolution. I have mentioned some of this already in this thesis. Fire enabled the cooking of meat, de-toxifying of many more species of underground storage organs, and preservation techniques such as smoking and drying of meat and fish, which provided us with the energy required for even larger, more expensive brains. Fire bestowed our ancestors with more time, by extending the period with usable light, and by lessening the time spent eating. (Chimpanzees spend hours chewing their food; cooking makes foods easily consumable and digestible.) Fire enabled the reduction of gut size, which did not have to work so hard to extract nutrients from our ancestor's diet, allowing reallocation of further energy into encephelisation. ${ }^{103}$ Fire provided heat, protection, and light, extending the time that could be spent communicating, socialising, planning hunts, repairing tools. It kept vermin at bay, it provided a means for hardening

\footnotetext{
${ }^{102}$ For example, the 'casual and opportunistic harvesting of resources at the edges of bush fires' (Gowlett et al. 2012, p. 705).

103 'Roasting meat breaks down protein and starch molecules making them more digestible and so acts as an external stomach that would have compensated for the trade-off in the size of expensive tissues between gut and brain' (Gamble et al. 2011, p. 121).
} 
(charring) the ends of wooden lances into useful, pointed tips, and may have assisted plantgrowth management. (See e.g., Wrangham 2009.)

Fire control and use involved a number of behaviours (future-directed planning, group-level cooperation, response inhibition) that epitomise the socio-cognitive co-evolution of ancient hominins: advances in working memory, episodic memory, theory of mind, collective intentionality (Twomey 2013). Dunbar (2014a) points out that large hearths need a lot of firewood, around 3okg per day, and gathering that wood would have very likely been a coordinated, cooperative enterprise. All, or at least many, members of the band were involved in some way or other. And hearths were social magnets (Barham 2013). All members of the band benefit from a campfire and all would have been drawn to it upon nightfall. And Dunbar (2014a) points out that social eating (for example, a satiating cooked meal around a campfire) triggers the endorphin system:

We feel warm and friendly towards those with whom we eat. This might explain why we find social feeding so important... Social eating of this kind seems to be universally important across all cultures, yet no one has ever stopped to ask why we do this... The obvious answer is social bonding. (Dunbar 2014a, p. 195.)

So indeed hearths were social magnets. Wiessner (2014) provides ethnographic examples. She points out that although flickering firelight extends the day, it does not extend the time in which foragers could engage in utilitarian activities such as hunting, foraging, or tool-making. Rather, it extended the time available for social pursuits at a time that would not conflict with subsistence activities. In Ju/'hoansi hunter-gatherers, firelit night talk and activities 'steer away from tensions of the day to singing, dancing, religious ceremonies, and enthralling stories... Night talk plays an important role in evoking higher orders of theory of mind via the imagination' (Wiessner 2014, p. 14027). Stories were often accompanied by background music (e.g., musical bows—discussed later). The groups' economic and functional concerns, as well as personal gripes, were put to the side as individuals gathered to make music, dance, or tell stories. This often closed social rifts and facilitated bonding. 
Gowlett et al. (2012) argue that from 400 Kya onwards, hearths were common enough to infer that a suite of associated social behaviours would evolve. So it is plausible that fire culture and social eating themselves gave way to a central theatre for the emergence of proto-music, ritual, dancing, storytelling, and theatrical performances, in the spotlight of the fire-all artsy behaviours more or less universal in human societies today, all across the globe. Our mobile ancestors by this time were central place foragers, more organised/centralised around cooking hearths (and cave areas to protect hearths during bad weather), and as big-brained hominins, they would become easily bored and restless, yet would have been intuitively creative, innovative and, importantly, social. So it is no wonder that activities would eventually arise which would have strengthened group identity, rehearsed coordinated action and increasing levels of intentionality/theory of mind, and, importantly, channelled and shaped emotions: music, dance, story/mime, and other artsy behaviours. Fire culture must have also provided a selective pressure for the concretisation of predominantly verbal forms of proto-language, as vocal musicality provided the medium for the switch. Flickering light from the flames would not facilitate effective gestural/manual communication (Dunbar 2014b), which in any case is not effective once group size increases above three or four individuals (see footnote 100).

\subsection{Late Acheulean proto-music}

We do not have direct material evidence of proto-music during the Late Acheulean (although we do have very suggestive evidence of a general proto-aesthetic sense, and presumably the ability to abstract, in the over-large handaxes discussed above). Yet the social brain hypothesis, and in particular Gamble et al.'s (2011) emphasis on the role of emotion, offers a framework through which some light might be shed on the matter:

With predicted community sizes of up to 120 , we should expect selection for mechanisms to amplify the emotional basis by which lasting social bonds were forged. One selection pressure for this is clear. With larger community sizes less time was spent together as dictated by fission and fusion to balance population to resources. (Gamble et al. 2011, p. 124.) 
So Late Acheulean proto-music, building upon the Oldowan/Acheulean developments in musicality (motherese, call mimicry, vocal grooming, lithic sound play, and so on), is a plausible response to such a selection pressure, emerging through cultural innovation and niche construction. As Gamble et al. (2011) say:

[Proto-]Music... is one obvious means by which the emotional strength of interaction between individuals can be enhanced through chanting and related practices involving dance and performance... both as a means of communication and as a way of amplifying emotions during social interaction (Gamble et al. 2011, p. 124.)

These authors point out that evolutionary accounts have rarely considered the role of the emotions. It is plausible that some form of proto-musical behaviour played the role identified by these theorists:

Hominin emotions, as Turner (2000) observes, were under selection to support stronger social ties since this is how complex social life evolves. An important connect to make is therefore between those higher levels of intentionality and the amplification and modulation of emotion... In other words, hominins had to become aware of conceptual emotional states that formed the basis for managing social relations (Gamble et al. 2011, pp. 124125.)

It is worth emphasising that proto-music fits the bill-a social form permitting emotional expression, socialisation, and cultural innovation that need not leave material traces, and thus is consistent with the technological stasis in the Late Acheulean material record. The idea is that an innovative process, analogous to the amplification of material culture/technology, took place in heidelbergensis, though in the development and expression of emotions, rather than in the development of utilitarian material technologies (Gamble et al. 2014) —and that proto-music is one outcropping of such developments. And as Gowlett et al. remind us: 
... the behavioural past is not defined by the extension of our artefacts but rather by the dated time-space in which behaving hominins undoubtedly existed. We must then model this larger whole as well as the details of specific recorded events. (Gowlett et al. 2012, p. 702.)

The social brain hypothesis and related research provides us with a framework for taking seriously the above conjectures. Our search for the origins and expansion of music does not begin at merely 40 Kya with the onset of European flutes in the Upper Palaeolithic, discussed in the next section. That's what we would say if we thought "what you see is what there was". Rather, I have argued the following. First, hominin musicality is a mosaic of ancient origins, which we can trace, with due caution, through the Oldowan and Acheulean. Second, the origins of behaviours resembling something worthy of the admittedly vague description "proto-musical" are to be found in the socio-cultural/ cognitive developments occurring, incrementally, during the Late Acheulean—say, from 400 to $500 \mathrm{Kya}$, in some proto-musical form, based on my argument from hominin sociocognitive co-evolution, the upgrades in technological production/proto-aesthetics, and using the date associated with more common and continual hearths as social magnets as a proxy. One avenue for further archaeological research is focusing on whether persistant fire control evidence corresponds with the presence of anything potentially of musical usage and looking to use-wear/experimental analysis to constrain the range of plausible inferences.

In my view, linking social expressive performance and human evolution with emotions, advances in technology, and a proto-aesthetic sensibility makes hypothesising the emergence of proto-music intelligible. New research on the evolution of the emotions may well provide indirect means for testing these ideas (see for example Peretz 2011 for a review of the neurobiology of musical emotions: evolutionary models are one direction for future research).

See Table 8.1 for an overview of the Late Acheulean stage until the incremental onset and concretisation of behavioural modernity: 
Table 8.1: Late Acheulean musicality

\section{Survival/natural selection}

1. Cognitive play was essential to our development; speculative use of theory of mind in pretend-play with sound foregrounds attentive capacities and tendencies for cooperation in other domains.

2. Social complexity (e.g., division of labour, cultural expressions, emergence of simple ritual) drives the importance of group bonding and social/group psychological identity during individual development.

3. Encephalisation plus proto-music and verbal-gestural language gives rise to greater (because more efficient energetically) memory capacity for symbolism/mythic culture (as per Merlin Donald). More complex modes of storytelling accompanied by proto-musical expression emerges.

\section{Sexual/status/social selection}

1. Increases in sociality mean that identity formation is all important for functioning within the group. This leads to proto-musical flair being a fitness-increasing aptitude in certain individuals (for the likes of mate attraction or social status points).

2. Control, production and performance of theatrical rituals that defuse/manipulate group emotionality give rise to sub-groups (proto-musical partners/collaborators; apprenticeships, "schools") resulting in greater modes of social complexity.

\section{Group/multi-level selection}

1. Emergence of seasonal festivals of musical/artsy competition. For instance, long distance trade networks of over $300 \mathrm{~km}$ started about 130 Kya (Marwick 2003), so before $250 \mathrm{Kya}$ it is plausible that simpler, shorter trade routes were in place, establishing the beginnings of inter-group proto-politics and economic negotiations, plausibly accompanied by proto-music and ritualistic aspects just as their modern versions are today. Musical competitions, for example, are welldocumented in the ethnographic record (consider, for example, the Inuit song duels discussed in chapter 6) and may be a very old phenomenon.

2. Spoken proto-language plus in-group accent/tone engenders social codes/markers for within-group communication and group identity when meeting with other groups. Music and adornment becomes the order of the day for personal identification within groups.

3. Psychologically powerful group-initiation ceremonies to ensure personal identification/commitment/"belonging" to group, emerge in due course. Emotively manipulative music is an essential part of the ceremony. 


\section{Stage Five: Mid/Upper Palaeolithic mythic/symbolic/oral culture}

The narrative has now reached the long stretch of behavioural modernity. Within this phase, fully-fledged languages, long-distance trade networks of over 300 km (from 130 Kya onwards—see Marwick 2003), musicians and musical instruments, sculptures and cave painters, body painting and ornamentation, burials including grave goods, shamans/priests and religion all incrementally appear, though not simultaneously, and not permanently from first appearance. ${ }^{104}$ Our ancestors utilised ochre for decorative effect as early as 165 Kya (McBrearty and Stringer 2007). Decorative adornments/ornaments/beads such as shells and animal teeth and bones were not far behind, from as early as 100 Kya (Stiner 2014), appearing, disappearing, and reappearing in the archaeological record (see e.g., Zilhão 2007), becoming more common and continuous over time (from 60 Kya onwards; Gowlett et al. 2012). Blombos Cave in South Africa revealed engraved ochre artefacts dated at around $75 \mathrm{Kya}$ (Henshilwood et al. 2009). As noted above, these very early markers of the artistic are quite unstable in the archaeological record; so perhaps unsurprisingly, in my view, behavioural modernity was an incrementally evolving, continual process building upon the Late Acheulean (800-250 Kya) scenario pictured above. ${ }^{105}$

Material evidence of representational cave paintings and figurines/sculptures appear from around 40 Kya (Lawson 2012; Pike et al. 2012), depicting large animals and water birds, as well as part-animal, part-human creatures. Romanian caves reveal paintings of animals such as rhinos, cats, buffalos and horses from around $32 \mathrm{Kya}$ (Tugman 2011).

${ }^{104}$ Behavioural modernity is commonly understood to encompass (i) symbolic cultural life, (ii) complex communication, (iii) skilled manipulation of raw materials—such as pigments (e.g., for paint use) and mammoth tusks (hollowing out ivory to create a flute is no guileless task!), which likely reflects (iv) skill specialisation and more complex division of labour, implying changes in socio-economic organisation, (v) communally shared, embodied beliefs about the world. In sum, then, (vi) fully-expressed, modern modes of simple + systematic + speculative cognition.

${ }^{105}$ See McBrearty and Brooks (2000), Sterelny (2011), d'Errico and Stringer (2011) for support for this view, which is in contrast to theories of a "great leap forward" cognitive revolution or creative/symbolic explosion, around 40 to 50 Kya (e.g., Klein and Edgar 2002; Klein 2013, 2009, 2000; Diamond 1992; Dunbar 1996; Chase and Dibble 1987; Pfeiffer 1982). That latter view is now largely out of favour and there is growing consensus for an incremental picture of behavioural modernity. In particular, d'Errico and Stringer (2011) stress the emergence, loss, and re-emergence of material signs of behavioural modernity in the archaeological record, from 250 Kya through 20 Kya, and I am broadly sympathetic to the view that they espouse in their paper, that is, that anatomically modern humans from 250 Kya were more or less cognitively modern all along, and that so-called "behavioural modernity" and cumulative culture are the result of niche construction, gene-culture co-evolution, environmental variability and population dynamics. 
The famous lion-headed man of Hohlenstein Stadel, the oldest known figurine, dates to around 40 Kya (Bailey 2013). Venus figurines, the oldest known fully-human representations, appear from around $35 \mathrm{Kya}$ (Conard 2009). The famous, iconic depiction of cave hyena (see Figure 8.1), in Chauvet cave, southern France, is now dated to 32 Kya.

The oldest known musical instruments—bird-bone (predominantly vulture radius or ulna) flutes and mammoth-ivory flutes from the Swabian Jura range in southwestern Germany (specifically, the caves Hohle Fels, Vogelherd, and Geißenklösterle)—appeared around the same time as the oldest cave paintings and sculptures: 40 Kya (Morley 2013; Higham et al. 2012; Conard et al. 2009). Another find at Isturitz in France comprises around twenty ancient bird-bone flutes, although the age of these flutes varies extensively, from 32-35 Kya, to 11-17 Kya (d'Errico et al. 2003). All are confirmed sapiens sites. And since it was around this time - 40 Kya - that our Cro-Magnon ancestors arrived in Europe, it appears that they brought the means and ability to make musical technology with them; the cave sites these sapiens inhabited ultimately proved to be very successful preservation houses for these ancient instruments. As I shall argue, musical technology must have a much older past, currently (and perhaps forevermore) hidden from the material record.

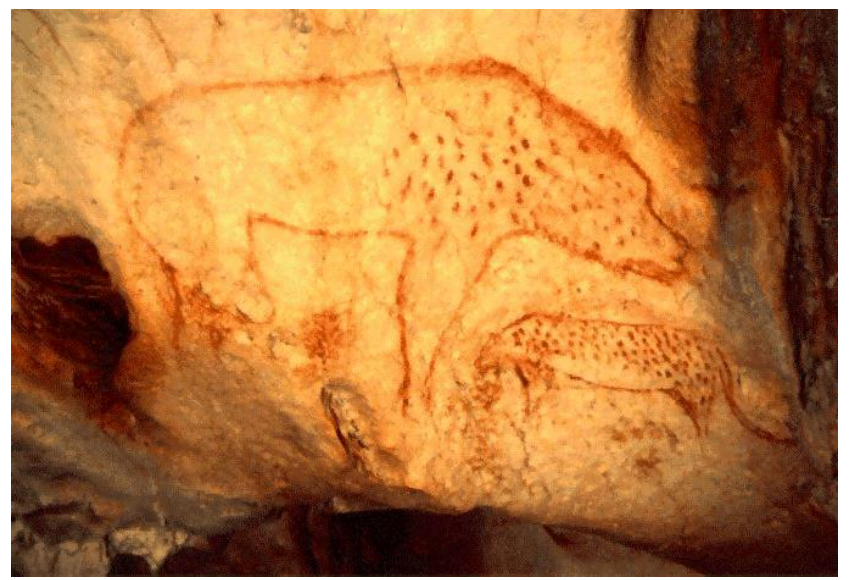

Figure 8.1: Cave hyena (Crocuta crocuta spelaea) painting found in the Chauvet cave ${ }^{106}$

\footnotetext{
106 Photo by Carla Hufstedler, freely distributable and adaptable under Creative Commons Attribution-Share Alike 2.0 Generic licence. Source= https://commons.wikimedia.org/wiki/File:20,000_Year_Old_Cave_Paintings_Hyena.png. Accessed 20/08/2016.
} 
One of the oldest undisputed flutes known (discovered in 2008 in Hohle Fels) is made from a griffon vulture (Gyps fulvus) radius: $21.8 \mathrm{~cm}$ long, with a diameter of $0.8 \mathrm{~cm}$. The body of the flute has been scraped smooth and fingerholes created from thinned-out concave depressions, pierced with the use of a tool. The proximal end of the bone has been manually adjusted: a hollow area (two V-shapes) was carved into the end of the bone so that it would function better as a mouthpiece (see Adler 2009; Conard et al. 2009). Cut-marks near the finger-holes suggest that their placement was measured, which suggests that the flute was created with either a pitch standard/scale, physical practicality, or pedagogy, in mind. Reconstruction experiments of prehistoric flutes from the Swabian Jura exhibit the wide range of tones possible on these instruments and establish them as 'fully developed musical instruments and not just whistles or pipes' (Conard and Malina 2008, p. 14). ${ }^{107}$

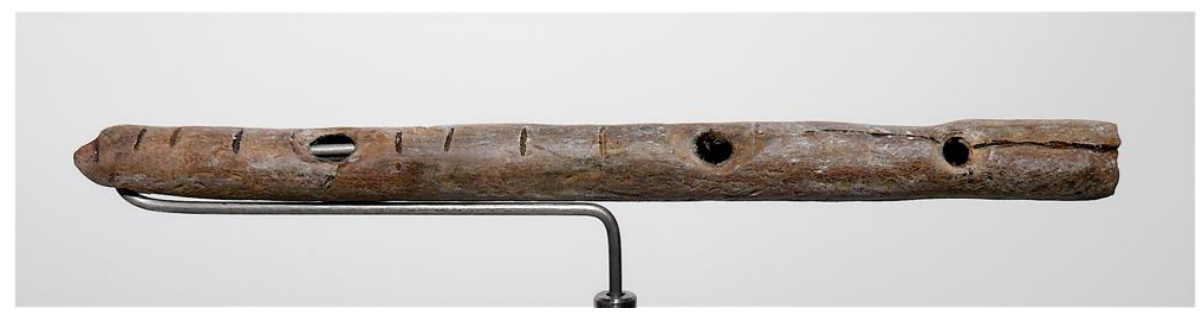

Figure 8.2: Replica of an Upper Palaeolithic flute from Geißenklösterle ${ }^{108}$

In my view, these sophisticated prehistoric flutes reveal a long history of instrument production and musical practice. As with the increasing sophistication of stone tool design from the Oldowan through the Acheulean, the construction of musical instruments further elaborates the advancement of human cognition and social learning. For example, they are

${ }^{107}$ See http://news.bbc.co.uk/2/hi/8117915.stm for an audio clip of a melody performed on a reconstruction of a prehistoric flute. Accessed 24/03/2014.

${ }^{108}$ Photo by José-Manuel Benito, freely distributable and adaptable under Creative Commons Attribution-Share Alike 2.5 Generic licence. Source= http://en.wikipedia.org/wiki/File:Flauta_paleol\%C3\%ADtica.jpg. Accessed 20/08/2016. 
crucial evidence of higher-order tool use: artefacts that were constructed with the aid of other artefacts.

Yet, we can ask, why do musical instruments suddenly appear only from 40 Kya? Why no earlier evidence? In my view, it comes down to a contingency of resources and preservation (Killin 2014). In the Upper Palaeolithic, avian fauna were very important subsistence resources for humans (Stiner et al. 2000; Cassoli and Tagliacozzo 1997). Moreover, vultures are not threatening to human hunters, are common in some environments, and would have provided more resources than just raw material for flutes (for instance, feathers for decorative effect, blood for use in ritual).

Vulture bones are sturdy, hollow, long and light, so they are especially suitable as raw material for flutes; the flutes would also be fragile, and perhaps would only survive in cave sites-many specimens are presumably lost forever to time's decay. Finally, flutes made from more ephemeral, easier-to-work resources (e.g., bamboo, cane, wood, or seaweed pipes) very plausibly could have predated bird-bone and ivory flutes and even co-existed with them.

The caves that contained these flutes for millennia proved to be excellent preservation houses. In general, bird bones preserve very poorly because of their light structure. Most of the flutes were discovered in fragments and pieced together by researchers; they had been used and discarded once broken or worn out, and their pieces were subsequently sheltered from the elements. Without the shelter provided by a cave, flutes made from hollow bone that were discarded or lost in earlier times, whether in Africa or in transit from Africa, very probably would now be lost to the elements. ${ }^{109}$

Indeed, we can be pessimistic that we should ever discover the oldest musical instruments (not that they would be a proxy for using extra-bodily adjuncts in the expression of musicality in any case). Consider that the ethnographic record contains a variety of alternative materials for flutes which are unlikely to preserve, let alone other musical instruments made from natural, ephemeral sources such as reeds, gourds, animal

\footnotetext{
${ }^{109}$ Even ostrich leg bones, which African hominins presumably had access too, are pneumatic.
} 
skins, and tree bark, not to mention ready-for-performance items that require little or no modification, such as bison horns, conch shells, logs, lithic material, and so on.

Several types of wind instruments - horn, wood, and ivory flutes and horns - that do not have finger holes, valve mechanisms, or the like, are common throughout SubSaharan Africa (Nettl 1990). Items such as these could have been used as musical instruments long ago, and even if we discovered them, we may not realise that they were used in that way. Modifications (such as a hole for blowing through) may be subtle, or not part of the surviving fragment of an otherwise unmodified animal horn (imagine an older variant on the African ivory horn depicted in Figure 8.3-an ivory horn unmodified but for a punctured blow-hole).

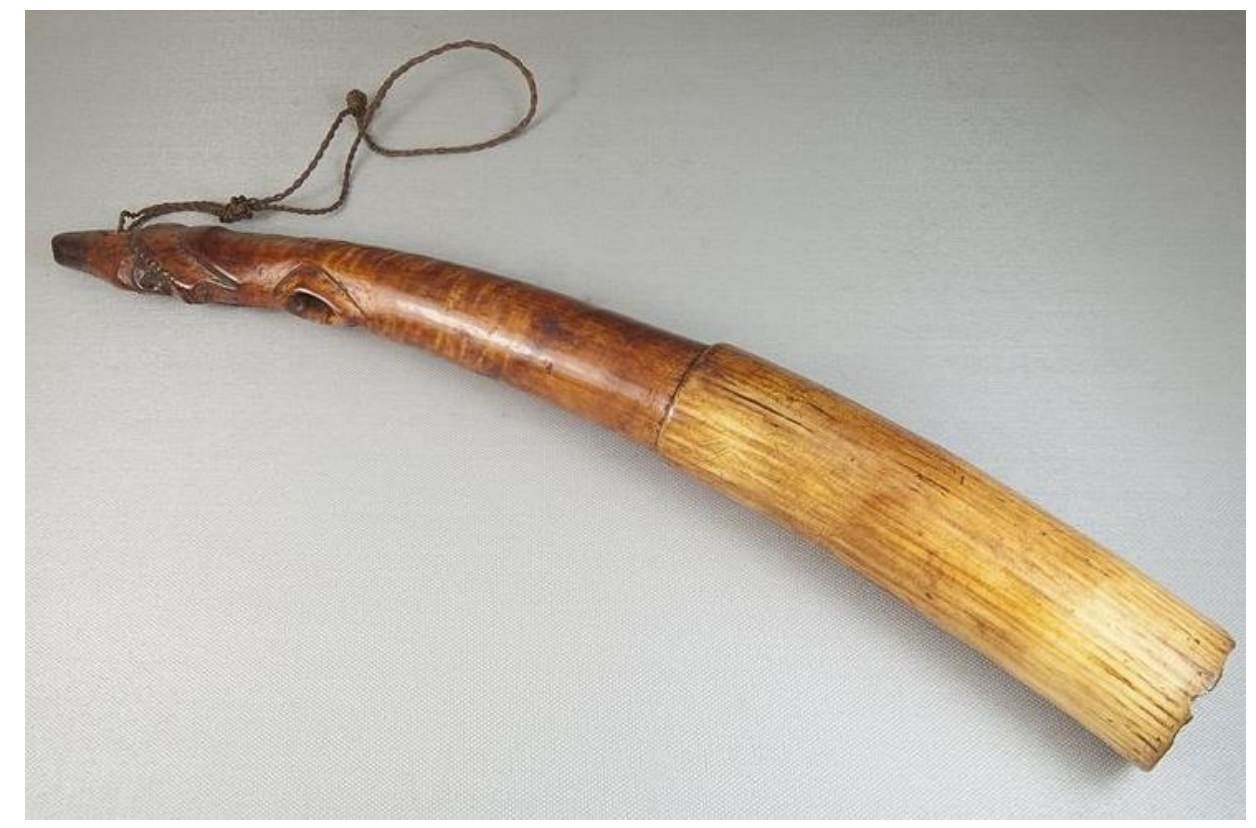

Figure 8.3: Central African ivory horn ${ }^{110}$

${ }^{110}$ Photo by Brooklyn Museum, freely distributable and adaptable under Creative Commons Attribution 3.0 Unported licence. Source=

https://commons.wikimedia.org/wiki/File:Brooklyn_Museum_22.1229_Horn.jpg. Accessed 21/08/2016. 
Recently some theorists have conjectured that materials such as Ecklonia maxia-a kelp common in many areas of Africa (Anderson et al. 1997) and used even today to construct natural flutes, trumpets, pipes and percussion—were used for producing various musical instruments before the onset of the Upper Palaeolithic flutes (Epsi-Sanchis and Bannan 2012). ${ }^{11}$ In any event, this stage represents a clear arrival of a musical prehistory that begins to resemble modern practices; the Upper Palaeolithic instruments at 40 Kya are not mere proxies for the culmination of music from musicality, but direct evidence of already fullyfledged music. There is good reason to think that our ancestors, by this stage, "had the knowledge, expertise, incentive and time to craft sophisticated objects for use in ritual activities. These activities probably served to affirm group affiliation, signal social identity and mark important social events or rites of passage' (Adler 2009, p. 695). The following discussions of music archaeology (\$2.1) and traditional/hunter-gatherer music ethnography (§2.2) aim to provide support for these claims.

\subsection{Music archaeology of the Upper Palaeolithic}

Prehistoric musical instruments enable rare and fascinating glimpses into an otherwise largely hidden culture, revealing more and more about our lineage's ancient past. Morley (2013) offers an up-to-date review and inventory of prehistoric musical instruments as yet unearthed by archaeologists, including 104 undisputed bird-bone and ivory flutes ${ }^{112}$, as well as whistles (pierced reindeer-foreleg phalanges), and purported sound-producers such as bullroarers, rasps and various forms of struck percussion. Kuhn and Stiner (1998), for example, identify a modified ungulate bone from around 32-35 Kya that is reminiscent of rasps found in modern traditional musical cultures. Its function as a musical artefact may be an educated guess, as is that of the alleged bullroarers and other such artefacts discussed by Morley, however, use-wear analysis might shed light on their presently murky status. ${ }^{13}$ Similarly, musical traditions/rituals could have co-opted utilitarian artefacts such as hunting

\footnotetext{
${ }^{11}$ See http://www.youtube.com/watch?v=AphqCZsWZxk for an intriguing video clip that demonstrates the performance and production of such kelp-based instruments, capable of extraordinary musical expression, yet incredibly simple in design. Accessed 24/04/2014. ${ }^{112}$ This is a conservative figure, which does not include contested items (such as the infamous Divje babe I "Neanderthal flute"-see footnote 99) and other items whose anthropogenesis or status/use as intentional sound-producer is controversial.

${ }^{113}$ As use-wear analysis has shed light on, for example, shell technology uses from the Upper Palaeolithic (see Cuenca-Solana et al. 2013).
} 
bows for use as musical instruments (Lawergren 1988). Contemporary hunter-gatherer bands in Sub-Saharan Africa use bows as musical instruments (Nettl 1990; Camp and Nettl 1955; see Figure 8.4). Similarly, the shafts of spear-throwers have been used as musical instruments by some Australian Aborigine tribes (Gould 1969); boomerangs are often used as musical clap-sticks (Stubington 2007).

Increasingly complex, composite hunting machinery appears in the archaeological record after 100 Kya, such as spears with darts, bows and arrows (64 Kya), and traps (Wadley 2010; Barham 2013). Our ancestors were creating artefacts that combine different resources and require specialist manufacture or tools to make them, which are also made up of multiple sources. They were certainly capable of crafting instruments that made pleasant sounds.

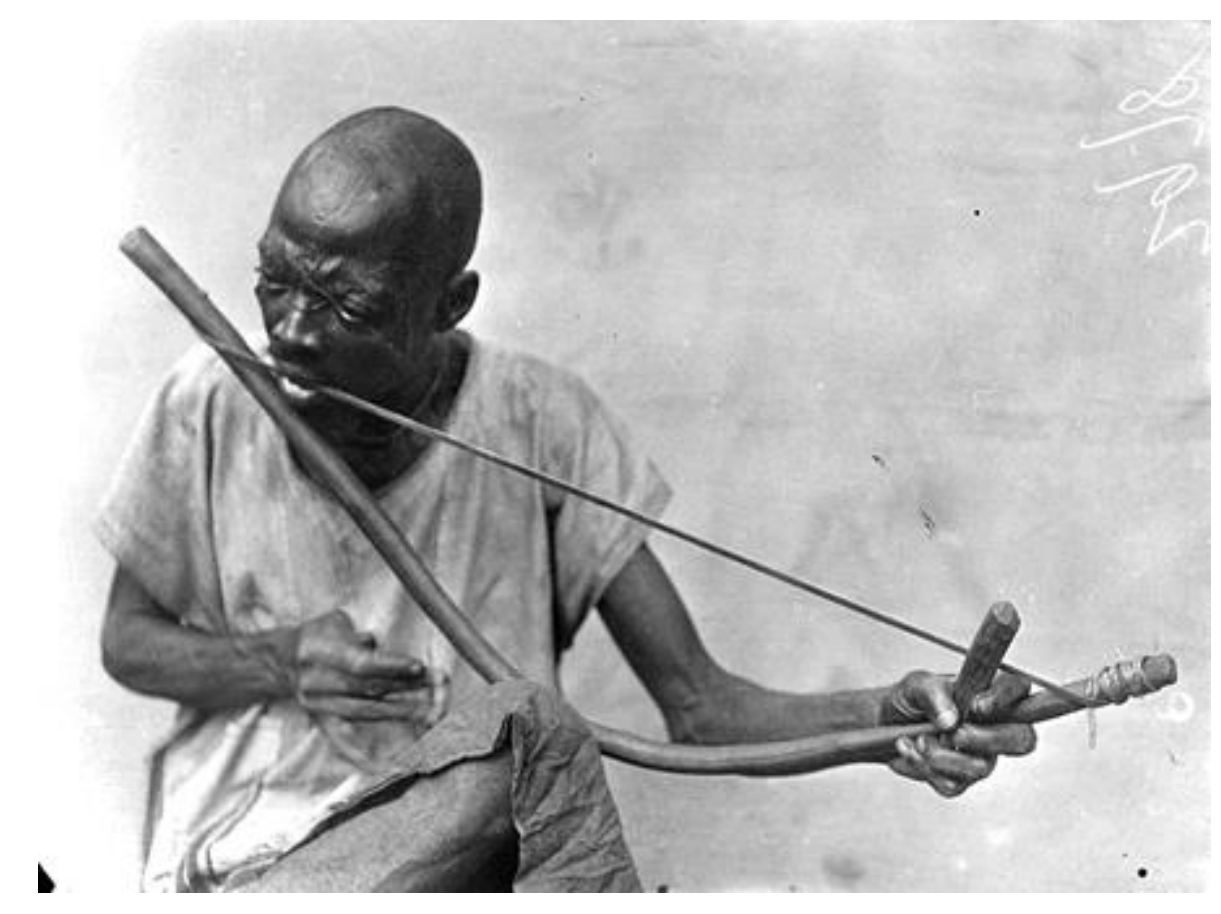

Figure 8.4: African musical bow (Obubra, Nigeria), using the mouth as a resonator ${ }^{114}$

\footnotetext{
${ }^{114}$ Photo from Northcote Thomas Collection, P.31234.NWT, now in the public domain. Source= https://commons.wikimedia.org/wiki/File:MusicalBow.gif. Accessed 20/08/2016.
} 
Consider the Swabian Jura mammoth-ivory flutes, of which the oldest is also dated back to around 40 Kya. Compared to bird-bone, the production of flutes from ivory requires greater skill, precision work and effort. So it is no surprise, then, that there are far fewer ivory flutes than bird-bone flutes in the archaeological record, even though they would probably preserve much better. Vulture and swan radius and ulna are naturally hollow and already an appropriate size, as well as light, sturdy and thus easy to craft; ivory is oversized, layered, and tough to work. Ivory flute production requires that:

... a section of ivory must be sawn to the correct length, it must then be sawn in half along its length, the core lamellae (layers) must be removed, and then the two halves of the flute must be refitted and bound together with a bonding substance which must create an airtight seal in order for the pipe to produce a sound. (Morley 2013, p. 50)

I follow Morley in assuming that the use of ivory as a raw material was considered valuable and significant—perhaps an honest signal of the producer's skill and investmentgiven the greater demands on working ivory, compared to bird-bone, into an item that is in many ways only equal to its bird-bone counterparts, although perhaps more durable. (Bird-bone flutes must have been very fragile, and with a potentially short lifespan.) Ivory flute production certainly bespeaks the maturity and sophistication of Upper Palaeolithic musical technologies - these were big ticket items - and the commitment of resources such as ivory to musical technologies implies that music really mattered to ancient hominins. We can safely presume that the producers and performers of these instruments knew what they were doing - that is, there were established musical traditions-they were not mere neophytes.

\subsection{Music in traditional/hunter-gatherer peoples}

It became a cliché in writings about the musical life of different traditional cultures to note that 'singing accompanies every moment of life in this society'. And truly, singing in many traditional societies literally accompanies every step of every single member of a society, from the birth of a person 
and mother's soothing lullabies, childhood game-songs, love songs, wedding songs, religious songs, hunting songs, agriculture songs, traveling songs, military songs, healing songs, finishing inevitably with funeral songs. (Jordania 2011, p. 76.)

Archaeology, due to the nature of the material record, can only present a certain partial kind of picture of Palaeolithic musical activity. The ethnographic record exposes a variety of ways of being musical with artefacts/resources that would not preserve or that require little, if any, modification. So following Morley, we can look to the musics of modern hunter-gatherer societies 'to examine and illustrate a wider diversity of the musical behaviours that exist' (Morley 2013, p. 12). My goal here is not to present a crude ethnographic analogy (cf. Bowra 1962). ${ }^{115}$ Rather, my goal is to survey the possible ways that our ancestors could have been musical that are actually instantiated in groups living in similar conditions, revealing some similarities in material resources used, predominant use of the voice (rather than musical instruments), and the social/group/affective nature of much music. Keep in mind that humans are niche constructers par excellence. Turning to the ethnographic record can help explore the ways in which music plays a role in processes of cultural niche construction (leading to potential fitness differences), and its roles in the social group and wider context.

There are all kinds of very important differences between ancient and ethnographically-known foragers, and in some contexts this important fact renders the kind of comparison I attempt erroneous. But I suspect those differences are unlikely to be relevant to the role of music in traditional social worlds, especially given how central it is to ethnographic foragers, so the survey is well worth having. My analysis of each case study will be, of necessity, brief and succinct.

\footnotetext{
${ }^{115}$ Bowra thinks that 'Modern primitives live the life of their Palaeolithic ancestors and have added almost nothing to it. Their songs are indeed songs of the Stone Age before it takes to agriculture and the domestication of animals' (Bowra 1962, p. 266).
} 
I begin by borrowing heavily from Morley's own analysis. Morley (2013) focuses on the music of four foraging societies that employ mobile hunter-gatherer subsistence strategies and lifeways, since their traditional practices do not result from the agricultural shift that characterises the Neolithic/Holocene, the next stage of the model. These are Native American, African Pygmy, Australian Aborigine, and North American Arctic groupssocieties that occupy diverse ecological niches and environments, and their evolutionary lineages are temporally as well as geographically widely displaced.

So although traditional/hunter-gatherer groups are not models for prehistoric Homo, I argue that some interesting parallels can be drawn: (i) material resource use/availability; (ii) predominant use of the voice, rather than musical instruments; (iii) the social/communal nature of much music. I add to these brief case studies a discussion of New Zealand Māori music and musical instruments. Māori people were not pre-Holocenestyle nomadic foragers in New Zealand prior to European settlement (at least, not to the same extent as the other groups under discussion-even historically, pā were major settlements), however I will argue the study of Māori music sheds light on these parallels nonetheless.

First, though, I should stress that while some aspects of these musics and their roles depend on the full suite of behaviourally modern socio-cognitive competences, quite a few do not. And the technology, and much of the communicative and cognitively-enabling conditions, very plausibly, would have been realised in hominins living by $250 \mathrm{Kya}$.

The Blackfoot and Sioux tribes of the North American Plains are nomadic foragers and hunters of bison and antelope. Their traditional music consists of monophonic song (that is, songs with a single melodic line), which are accompanied by unpitched percussion such as rattles, drums, bullroarers and rasps. Religious/ritual activities, social dancing, war dances and puberty rites (for group initiation) all involve the production of music. Music is a communal affair-everyone is involved to some extent. Their songs often comprise vocables —expressive vocal sounds (non-lexical syllables) — rather than words. The use of such songs used in connection with symbolic activities like rituals and rites is, for the most part, not symbolic or propositional. Rather, their use contributes to the context at hand emotionally (McAllester 1996). Some traditional songs, however, are of a more symbolic 
nature. Blackfoot sun dances, for example, are believed to beseech vigour, wellbeing and prosperity ('Sun says to sing', Morley 2013, p. 18). Moreover, sun dances have social functions: "bringing the tribe together and permitting social interchange, gambling, and athletic contests' (Nettl 1990, p. 180). Other songs are more iconic. As discussed already in this thesis, the "bleating calf" song is part of the Blackfoot hunting toolkit: the musicohunting strategy is to lure a herd to the top of a cliff face, and then force them over; that way, gravity does the difficult work (see Kehoe 1999).

By skilfully mimicking a lost calf in his actions and by bleating pitifully, the caller attempted to make the bulls that led the herd follow him. Taking a zigzag course so as not to make them suspicious, he would gradually lure them into the funnel [of an impoundment], quickening his pace all the while. Once the herd was well into the chute, tribes-men concealed behind the stone and brush piles stood up, shouted, and waved their robes to stampede the bison at the rear end of the herd. These frightened beasts would surge forward, impelling the leaders of the herd into the pound, willing or not. The buffalo caller quickly dodged to one side to save himself from being trampled to death, and made his escape. Sometimes a convenient steep bluff or gully was substituted for the pound, the bison breaking their legs as they fell over the precipice or being trampled by those behind as they crowded into the narrow gully. (Howard 1984, p. 61.)

The musical instruments of the Blackfoot and Sioux are primarily unpitched percussion instruments, including split wooden sticks, wooden rasps, bullroarers, hand drums (a stretched animal skin membrane over a wooden frame), cocoon leg- and anklebracelet rattles, and other rattles made from cocoons, deer hooves, gourds, and turtle shells. A few melodic instruments do feature, however, including wooden and bird-bone whistles and elder-wood flutes (see Morley 2013; for a detailed discussion about Native American musical styles, see Nettl 1990, chapter 8). Note that very few of these items would generally be considered candidates for preservation. 
Similarly, the Aka and Mbuti pygmy tribes of equatorial African rainforest produce music using vocables rather than words-lacking in symbolic content, and contributing to contexts emotionally. These nomadic tribes occupy dense, humid forest environments, in contrast to some other pygmy groups (for example the Efe and Baka) who live nearer to villages or farmers and have established more significant barter systems with those people, trading services for food. Aka and Mbuti music is also predominantly vocal, with bodypercussion and handclapping. And as above, music is a communal affair in which all folk participate (to varying extents). As well as group-based music, there are lullabies, sung one-on-one by a carer to an infant or child.

These pygmy groups' musical instruments include wooden sticks, various rattles, end-blown flutes made from cane, and drums made from animal skin membranes stretched over hollow logs. They are thus similar to the suite of instruments, unlikely to preserve, found in the musical traditions of the Blackfoot and Sioux-despite great variation in the musical styles between the Aka and Mbuti on the one hand, and the Blackfoot and Sioux on the other (Aka and Mbuti vocal music tends to be polyphonic, for instance, which involves several different melodic lines occurring at the same time; Blackfoot and Sioux tends to be monophonic).

There is some contrast between these groups and the Aboriginal groups of Australia. Australian Aboriginal societies have a highly symbolic, lyrically-dominated song tradition tied closely to geography, land ownership, traditional story-telling, and ritual (Tjukurrpa, 'The Dreaming'). (Of course, not all Aboriginal vocal music has lyrics: consider the post-death ritual vocal mourning described by Ellis 1985 and Stubington 2007, a kind of pitched wailing). Traditionally, ownership or possession of a song is a highly valued signifier of social status: 'the most knowledgeable person in a tribal community was the person “knowing many songs"” (Ellis 1985, p. 1). That is:

... song is one of the most important vehicles of [Australian Aborigine] communication... Through song the unwritten history of the people and the laws of the community are taught and maintained; the entire physical and spiritual development of the individual is nurtured; the well-being of the group is protected; supplies of food and water are ensured through musical 
communication with the spiritual powers; love of homeland is poured out for all to share; illnesses are cured; news is passed from one group to another. (Ellis 1985, p. 17.)

Many Aboriginal songs, including lullabies, express creation tales of god-like ancestors shaping land structures and forming animals-preserving their mythologies and traditional cultural folklore. Other songs map their vast, barren desert environment-a useful mnemonic device. ${ }^{116}$ So unlike the Blackfoot, Sioux, Aka and Mbuti, much cultural/ geographical/ecological information is contained and expressed through music. However, similarly, their music is predominantly vocal with clapping/body percussion and simple technological additions that vary from region to region: sticks that are struck together (sometimes boomerangs, co-opting a hunting tool for musical purposes), bamboo whistles, didjeridu, leaf-, seed-, and shell-rattles, rasps, animal-skin drums, and bullroarers (Stubington 2007). Didjeridu, traditionally a termite-hollowed wooden tube that is blown to produce a powerful drone with a rich variety of ornamentative flourishes, is a culturally significant musical instrument that traditionally imitates animal calls and other natural sounds. (Of course, the instrument has now been widely appropriated into Western and fusion musics.) Bullroarers have been utilised variously in ritual, as well as music performance contexts, demonstrating how musical items have been co-opted into the expression/manifestation of traditional worldviews. For example, Sachs (1962) writes of at least one traditional Aboriginal group, that:

... the bull-roarer, as a ghost's voice, frightens the women away when boys are being circumcised in the bush; a woman who has happened to see the instrument must be killed. Indeed, the bull-roarer is supposed to hold the forces of ancestors and to fecondate the wombs of women (Sachs 1962, pp. 96-97.)

\footnotetext{
${ }^{116}$ In fact, this understates it. Stephen Davies points out to me that Aboriginal song sequences can contain over a hundred songs, the totality of which provides a detailed map from one site to another.
} 
In short, music plays a substantial role in Aboriginal socio-cultural life, to say the least. Children that grow up in a social environment in which much information is expressed through traditional song—such as, for instance, Australian Aboriginal society that traditionally maintained a highly symbolic, lyrically-dominated song tradition tied closely to geography, land ownership, story and ritual, and which maps their vast, barren, homogenous desert environments and preserves creation mythology and traditional folklore through song-develop in a context that is scaffolded by these songs and their role in informational transmission and social learning.

Writing in the 1950s, Waterman notes:

Throughout his life, the Aboriginal is surrounded by musical events that instruct him about his natural environment and its utilisation by man, that teach him his world-view and shape his system of values, and that reinforce his understanding of Aboriginal concepts of status and his own role. More specifically, songs function as emblems of membership in his moiety and lineage, as validation of his system of religious belief, and as symbols of status in the age-grading continuum. They serve on some occasions the purpose of releasing tensions, while other types are used for heightening the emotionalism of a ritual climax. They provide a method of controlling, by supernatural means, sequences of natural events otherwise uncontrollable. Further, some types of songs provide an outlet for individual creativity while many may be used simply to conquer personal dysphoria. (Waterman 1956, p. 41.)

And Malm notes:

Music is used throughout an Aboriginal's life to teach him what he must know about his culture, about his place in it, and about its place in the world of nature and supernature. As a baby he is encouraged to dance and sing about everyday tasks. At puberty he learns his first karma songs-about the totemic plants and animals of his clan and the history and mythology of the 
group-which belong to his lineage... In the bachelors' camp he learns more light-hearted songs which are the basic entertainment media for the band... His maturation can be measured in the esoteric knowledge he has acquired through song, and as an old man he knows that his honor is based partly on his mastery of the secret sacred songs of the band. (Malm 1967, p. 2.)

Many Aboriginal songs are whole-community songs; Ellis calls these 'open songs' (Ellis 1985, p. 55). These form the backbone of Aborigine music repertoire. Other songs, like particular ritualistic songs, or children's songs-both songs for children and songs by children-are 'closed songs', which might reveal an aspect of social life or religious doctrine deemed appropriate for only 'selected members of the tribe' (Ellis 1985, p. 57). Ellis discusses one such closed song which tells of a young girl's reaction to seeing her brother's post-initiation modified penis. Closed songs such as this may be restricted because of their explicit or anti-social subject matter, but the fact that songs are restricted at all, in Ellis's view, is because it gives power-and affords respect—-to the elders and people of high standing. Knowledge of songs, and control over songs, are attributes held in very high esteem.

While some songs may be tribe- or region-specific, others spread long distances, via trade networks and other cross-group interactions. E. H. Davies (1947) provides an account, before the widespread establishment of playback technologies from the 1950 s onwards, of an Aboriginal song that was recognised when a recording of it was played to a group of Aboriginal musicians living $1,600 \mathrm{~km}$ from the place the song was recorded. However, for the most part, like Aboriginal languages, music differs markedly from group to group (Ellis 1985).

The music of the Yupik of southwest Alaska is also predominantly vocal (Morley 2013). Like Australian Aboriginal song, it is lyrically-driven. The voice is accompanied by the likes of frame drums (sea-mammal skin stretched over a wooden frame, made by specialist drum-makers) and puffin-bill rattles. Yupik songs cover a diverse array of contexts and uses, 
including ritual, children's games, animal identification, and hunting, travelling and foraging songs. Thus like Australian Aborigine music, Yupik songs contain and express much cultural, social, and ecological information, perhaps again because they live in such a challenging environment.

The same is true of the music of many other indigenous Inuit peoples in Alaska, Canada and Greenland (Diamond 2008). Sachs (1962) writes that it is:

... hardly an exaggeration to call the Eskimo a genuine people of musicians; every Eskimo must know the art of composition; and, in supreme contempt, a jealous Eskimo woman would sneer at her rival: "She can't dance, she can't even sing..." (Sachs 1962, pp. 137-138.)

Singing is also a form of competition among people in some Inuit groups. The anthropologist Jean Briggs describes how music took on a social management role in the development of large-group communities from small-camp nomadic lifeways since the 1960s. Hostility among adults was publically ritualised and managed through song duels (Briggs 2000), discussed in chapter 6 of this thesis. ${ }^{117}$

Finally, taonga puoro (New Zealand Māori musical instruments) are predominantly blown instruments of various styles, rattles, struck percussion, and bullroarers, made of bone, wood, shells, gourds, stone, leaves and reeds (Nunns 2014; Flintoff 2004; McLean 1996). (See Figure 8.5.)

After the decline of traditional Māori musical instruments upon the arrival of Europeans in New Zealand and subsequent colonisation (European missionaries discouraged Mãori from using traditional instruments, and those instruments were eventually replaced with Western instruments such as acoustic guitar, for accompanying traditional Mãori song and dance), the traditional instruments all but eventually disappeared in the twentieth century except for a few museum and collection pieces.

117 Somewhat similarly, demeaning songs sung about oneself by other individuals is a form of derisive gossip/punishment for sexual transgression in traditional lgbo (Afikpo) culture (Ottenberg 1989; Paul 2015). 


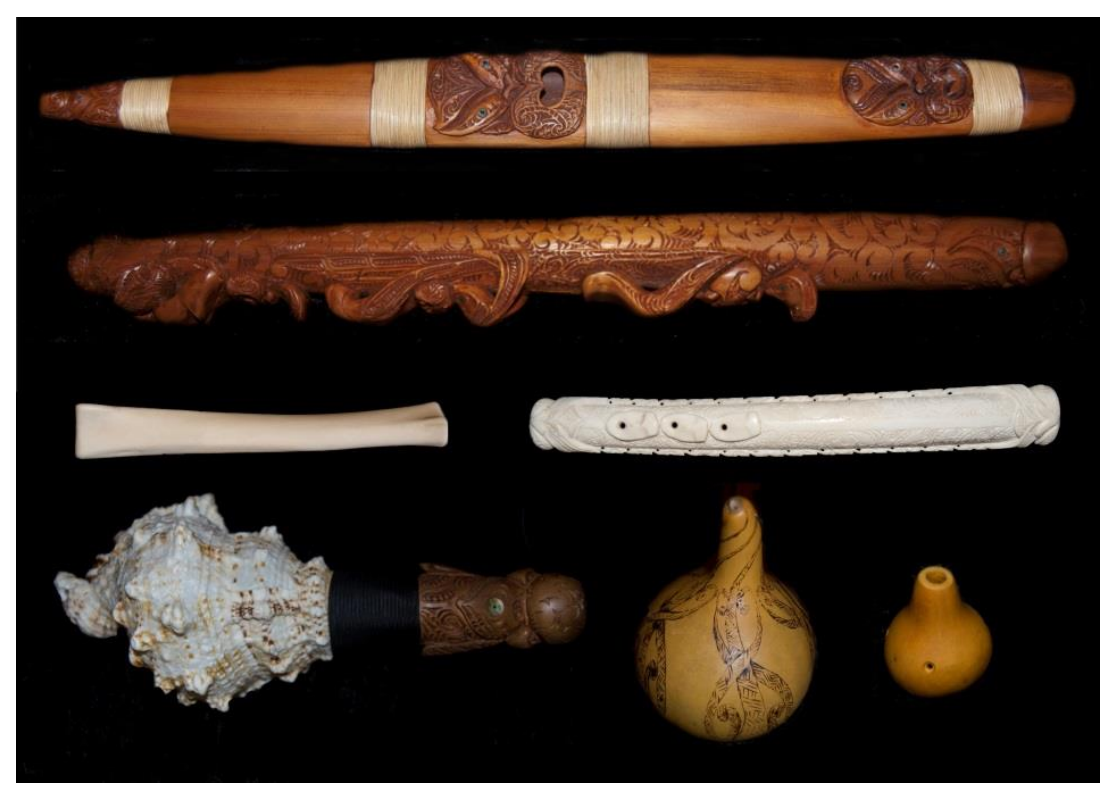

Figure 8.5: A selection of Māori instruments ${ }^{118}$

More recently, as detailed in Nunns (2014), a revival research and construction/performance programme championed by the trio of Richard Nunns, Brian Flintoff, and the late Hirini Melbourne has seen the reconstruction of many traditional instruments and the collection of childhood memories of Mãori elder informants, in a bid (1) to enable a new generation of performers to recreate the sounds of the instruments as authentically as possible (given the lack of formal cultural transmission of the music during the twentieth century), and (2) to rediscover the cultural functions of the music in traditional Mãori lifeways. Indeed, it appears skilled Mãori musicians played their instruments to entice birds for capture, to ease the suffering of those undergoing traditional tattooing procedures and women undergoing labour pains/child-birthing, to seduce a desired lover, to attempt to aid conception, pregnancy/gestation, and the ossification of a new-born's fontanelle, to install information of tribal heritage in new-borns, to accompany ritual, to express sorrow and loss during funeral events, to encourage healing, to entreat spirits, to send long-distance

\footnotetext{
${ }^{118}$ Photo by Declan Cudd of Rotorua Museum, freely distributable and adaptable under the Creative Commons Attribution-Share Alike 3.0 Unported license. Source= https://commons.wikimedia.org/wiki/File:A_selection_of_Taonga_p\%C5\%ABoro_from the collection of_Horomona_Horo.jpg. Accessed 20/08/2016.
} 
messages/signals, attract attention, or announce presence, and, of course, to entertain, accompanying song, dance, and spoken words.

In spite of the decline of Māori musical instruments, traditional Mãori songs have continued to be highly-valued and taught to new generations. These songs have traditional functional categories, allowing a tantalising glimpse into the importance, and uses, of Mãori music in the past. This includes karakia (prayers/spells/charms/incantations), tauparapara (introductory chants that precede a speech or informal address, including to welcome guests or to farewell the dead), whakārāra pà ('call to arms' songs sung by sentries to announce the approach of rivals), pätere (songs written and sung by women in response to slander/gossip), kaioraora (virulent songs expressing hatred and anguish, for instance over a slain partner, directed towards the guilty party), haka (shouted, aggressive war songdance, popularised by the New Zealand All Blacks rugby team), various funeral songs, women's songs, welcoming and announcement songs, ceremonial/ritual songs, foodbearing songs, work songs, hauling songs, paddling songs, laments and love songs, curse songs, genealogical songs, instruction songs, various game songs, and many others besides (for fuller detail, see McLean 1996).

Time to take stock. There are several upshots from these brief ethnographic case studies. Despite great variability in musical style cross-culturally, traditional/hunter-gatherer music is predominantly vocal, with body percussion and percussion instruments made from organic, ephemeral resources that typically would not be candidates for archaeological preservation. So perhaps it should be wholly unsurprising that the earliest material evidence of musical instruments dates back to only 40 Kya. The period 250-20 Kya, in my view, must have contained a variety of musical instruments (culminating in the bird-bone and ivory flutes of the Upper Palaeolithic), many of them perhaps not unlike those discussed above, and may be forever lost to the archaeological record. This is almost certainly confirmed by the sophistication and design of the musical instruments that appear from 40 Kya onwards: it is near inconceivable that musical technologies could have started just like that from the get-go.

Hunter-gatherer songs 'are basically “tools” for invigorating life' (Barac 1999, p. 435). In hunting, songs are used to attract game, raise the confidence of hunters, and, as 
a group, celebrate a successful hunt, or lament a hunting failure. Other songs are linked to rites that make up the human cycle (Bowra 1962): birth songs/lullabies, and songs for circumcision, puberty, love/marriage, sex, sickness/old age, death. Yet others attempt to influence the world, perhaps via the spirits, such as Inuit weather songs, or Vedda songs for enchanting hunting arrows (Bowra 1962). Both carer-to-child lullaby and group-based music widely feature, as well as, in some cases, music that encodes specific information like cultural histories and myths, and natural geography/ecology.

While there may be specialist instrument makers, and high-status composers or owners of songs (perhaps some of them apparently receiving them from divine communication), group-based music is typically not performed by elite specialists for passive audiences - it is a whole-band social affair, often tied up emotionally in ritual or other social contexts. And forager groups tend to expect a high degree of compliance and participation in their communal traditions and rituals (Hewlett and Cavalli-Sforza 1986; Barham 2013). As Sachs writes: 'The neat separation of amateurs and professionals, a pillar of modern musical life, presents a concept not applicable [here]' (Sachs 1962, p. 200). Of the pygmy tribes, to take an example, ethnomusicologist Joseph Jordania notes:

Every member of Pygmy society is a brilliant singer who can sing in harmony and participate in traditional choral singing with complex yodeling technique. Despite their amazing musicality, pygmies do not have any professional musicians. (Jordania 2011, p. 13.)

Some, but little, melodic instrumentation is evident (e.g., flutes), which represent but only a small part of the musical tradition at hand. Rather, the voice and percussion tend to dominate. This is really quite a striking fact. Moreover, these case studies confirm that music need not always be symbolic, so the presumption that music came online only recently—after symbolism and abstract thought—is not at all justified. As my discussion has revealed earlier on, ancient hominins have long been competent artefact producers and users - from crude stone tools to oversized handaxes and complex javelin-style weaponry. Yet many of the hunter-gatherer musical instruments discussed here require little 
modification: much less work than even the mammoth-ivory flutes of the Upper Palaeolithic. Musical instruments, in my view, represent a long tradition of resource modification and social learning that ultimately gave rise to the prehistoric flutes, whistles and other instruments preserved; very little else could be expected to preserve as clear markers of music played. I am thus sympathetic to hypotheses such as that of Epsi-Sanchis and Bannan (2012), who place a tradition of kelp-based musical instruments at 80 Kya in Africa (see discussion above; see also footnote 111) — the burden is perhaps now squarely on the sceptic to show that musical instruments came late; that what we see in the archaeological record is truly all there was, or is all we are justified in talking about.

\section{The final episode: the Holocene (Neolithic, Bronze Age, Iron Age, classical antiquity)}

Our Pleistocene ancestors were musical. Although the flutes dated to 40 Kya may well be evidence of specialist instrumentalists, for the most part, and for a long time, much music was vocal/percussive, created by people who were not music professionals (that is, if the music of contemporary foragers can be used as a guide); it was music that everyone in the group could recognise and comprehend, allowing full-group participation. But this was to change, at least for art and popular music of the post-agricultural Holocene world. As Nettl notes:

Then, among some peoples, there must have taken place the development of a separate musical life for an educationally sophisticated and economically or politically powerful segment of the population, while the rest of the people held on to the older [group-based] musical tradition. In Western civilisation, we tend to be dominated by this more sophisticated musical culture, which includes our concert music and also the vast body of popular music. (Nettl 1990, p. 2.)

In this section, I argue that the emergence of this new musical life occurred in, and was enabled by changes in, the Holocene world: the Neolithic transition through to the 
Common Era. In short, here I discuss the musical revolution, of some specific regions of the world, between roughly 10 Kya and 2.5 Kya.

As global temperatures rose in the wake of the last Ice Age, around 10-12 Kya, tundra and ice caps receded, giving way to plains and forests. In multiple geographic regions, the lifeways of many human groups switched from nomadic/forager to sedentary: societies with farming and agriculture, increased storage capabilities, and greater harnessing of natural resources (Testart et al. 1982; Renfrew and Bahn 2012). Farming was established in the Near East by 10 Kya, spreading to Europe, South Asia and Pakistan by around 8.5 Kya (barley, wheat, goats, sheep, and eventually cattle from 6 Kya). Independently, millet and rice cultivation was established in various regions of China and Southeast Asia by 7 Kya. (This eventually made its way to Africa; sorghum wheat and millet cultivation was established in the Sahara by 5 Kya.) Also independently, in the Americas, various vegetables (peppers, squash, beans, and later also potato and manioc/cassava) were being farmed by around 9-10 Kya in Peru, Mesoamerica, and other South American regions. Later, maize was farmed in Mexico and Argentina by 7.6 Kya. (For more detail see Renfrew and Bahn 2012.) Eventually, the world's remaining hunter-gatherer bands came to be greatly outnumbered by agricultural societies. Only a few continue to exist today.

This pervasive agricultural revolution, the so-called "Neolithic transition", supported bigger populations and greater social complexity: 'By $8000 \mathrm{BC}$, settlements like Çatal Hüyük... in Turkey and, later, Jericho in the Levant had become significant walled towns covering as much as 15 ha with 1,000 dwellings and perhaps as many as 5,000 inhabitants' (Dunbar 2014a, p. 305). Over the next few thousand years this was to spread throughout the Neolithic world. And innovations such as pottery, writing, metalworking, and the like arose—signs of craft specialisation through market size. Yet living in such dense communities gave rise to a whole new suite of stresses, selecting for mechanisms to relax those stresses and promote social bonding in significantly larger social worlds. Perhaps one such mechanism was the advent of brewing and social boozing (Neolithic pottery contains traces of alcohol dating back to 7-9 Kya-see McGovern 2009). Social drinking is universally a social bonding activity that triggers strong endorphin effects (Dunbar 2014a), which almost certainly would have included music in some way, as it invariably does today. 
Even so, musical social bonding would continue and eventually give rise to musical expression as hedonic entertainment (e.g., for elites) and increasing standards of artistry. Indeed, as the musical archaeological record indicates (which I go on to explicate in the following), it was during this period-in particular, between 2-5 Kya-that in the ancient civilisations of various regions of the world, musics qua art forms (in the vernacular sense of "music as art" - that is, specialists performing for a non-participatory audience), as well as many participatory folk music traditions, emerged from our ancestors' earlier forms of fullgroup participatory music and proto-musical performance/ritual/dance. ${ }^{19}$

Whereas mobile foraging societies are generally egalitarian, with no norms of political leadership and little, if any, heritable material wealth, the transition to sedentary life enabled the emergence and establishment of unequal hierarchies, elites, and privileged political authorities. After a period of intermediary chiefdom-based ranked societies, state societies and hierarchical civilisations emerged by around 5.5 Kya (Renfrew and Bahn 2012). Chiefs became kings and pharaohs and emperors; shamans and witch-doctors became priests/priestesses; equals became peasantry. The ever-growing sophistication of musical instruments and musical systems, in conjunction with a more hierarchical social structure, seems to have advanced a dividing performer/audience conception, and saw the technological advancement of more sophisticated forms of musical instrumentation implying specialisations (in both technical construction and musical performance mastery).

In my view, many musics ultimately modelled the hierarchical societies in which those very traditions were developed; once a fully participatory pastime, music came to be performed by specialists for a passive audience, and continues to exist in this format today. From art composers and orchestras to rock and pop groups, musicians produce and their fans consume and admire.

Thus to conclude my co-evolutionary, niche construction narrative I ought to survey music's Neolithic career. This will provide a picture of how music was shaped in various parts of the world in the final stages of its emergence (and "release", as art object) from

\footnotetext{
${ }^{119} \mathrm{~A}$ parallel transition seems to have happened in religiosity at this time, as older animistic/embodied full-group participatory religions became ones of doctrinal specialisation with particular individuals leading a prayer or ritual, or acting as a conduit for supernatural enlightenment, communication or cajolement on behalf of the group (Dunbar 2014).
} 
our lineage's deep musicality. Neolithic bone flutes, and turtle-shell shakers containing pebbles, discovered amongst the burial grave goods unearthed at Jiahu, China, have been dated to around 7 Kya (DeWoskin 1998). These flutes reveal a marked increase in musical sophistication compared to those of the Upper Palaeolithic. The flutes were all in pairs; nine pairs discovered in total. The flutes of each pair were tuned roughly one whole tone (major second) apart, with one of the flutes in each pair being slightly longer than the other.

DeWokin continues:

The flutes are carefully crafted and well tuned, showing a tuning precision that indicates sophisticated divisional measurements were done to position the fingering holes prior to drilling. The flutes are seven-hole, eight-tone vertical instruments... The hole-to-hole measurements are precise divisions apparently made by applying a measuring algorithm or through the use of a template. The adjustment made by the small addition of a tuning hole over the lowest fingering hole responded to variations in pipe diameter and shape and suggest that some kind of water-level tuning process was used empirically after the primary holes were laid out and drilled. (DeWokin 1998, p. 106.)

The water-level tuning method suggested here is assumed, but plausible. In any case, it certainly seems plausible that the uniform pairing of flutes across tombs, and the consistent intervallic and length differentiation across pairs, is evidence that the flutes were performed together, with the musicians probably playing different musical parts (given the major second interval gap between pairing flutes), perhaps in interlocking style. This image stands in some contrast to that of a solo flute accompanying voices and/or percussion instruments or body percussion—the basic image of Upper Palaeolithic musical instrumentation. 
By the time of the Ancient Egyptians (from 3,100 BCE until around $300 \mathrm{BCE}$ ) ${ }^{120}$, we know from surviving relics that:
... the playing of music was closely associated with the exercise of power and homage, with religious and secular rituals, and with state ceremony, dancing, love and death. These pieces of art depict a variety of instruments, from the simple sistrum or sekhem-a hand-held, U-shaped shaken percussion instrument- to harps, ceremonial horns, flutes and wind instruments whose sound is made by blowing across strips of reed... They also depict expert performers of high status, including members of royal dynasties and deities. The prevalence of music in Ancient Egyptian life is demonstrated by the fact that over a quarter of all the tombs at the necropolis found at the site of the city of Thebes are decorated with iconography of music-making (Goodall 2013, p. 8.)

Tombs and temple walls (for example, see Figures 8.6, 8.7 and 8.8) depict musical scenes including that of singers, harpists, flautists, players of reed wind-instruments (resembling clarinets/oboes), lute and lyre players, drum and tambourine players, small groups of performers, clapping/dancing females, often accompanying processions or providing music at public feasts. One relief shows a woman beating a tambourine in an effort to spook birds out from the undergrowth. Another shows a stick-beating performer leading a funerary procession. As well as reliefs, texts, sculptures, and other artefacts that depict musicians and musical instruments, archaeologists have discovered-from various moments in ancient Egyptian antiquity-drums, harps (of various shapes), lutes, cymbals, crotales, sistrum (shakers), tambourine membranes, trumpets, and clapping sticks (Manniche 1991). Such instruments as lyres, lutes, early oboes, and tambourines appear in the material record from around 1,800 BCE onwards (Healey 1994). For instance, KV62 (Tutankhamun's tomb) contained musical instruments sheltered from the elements for over 3,300 years (Renfrew and Bahn 2012).

\footnotetext{
${ }^{120}$ That is, between roughly $5.1 \mathrm{Kya}$ and 2.3 Kya. As dates are nearing the present I will follow archaeological convention and provide (still approximate) dates in "BCE" rather than "Kya/Mya" format (see e.g., Renfrew and Bahn 2012).
} 


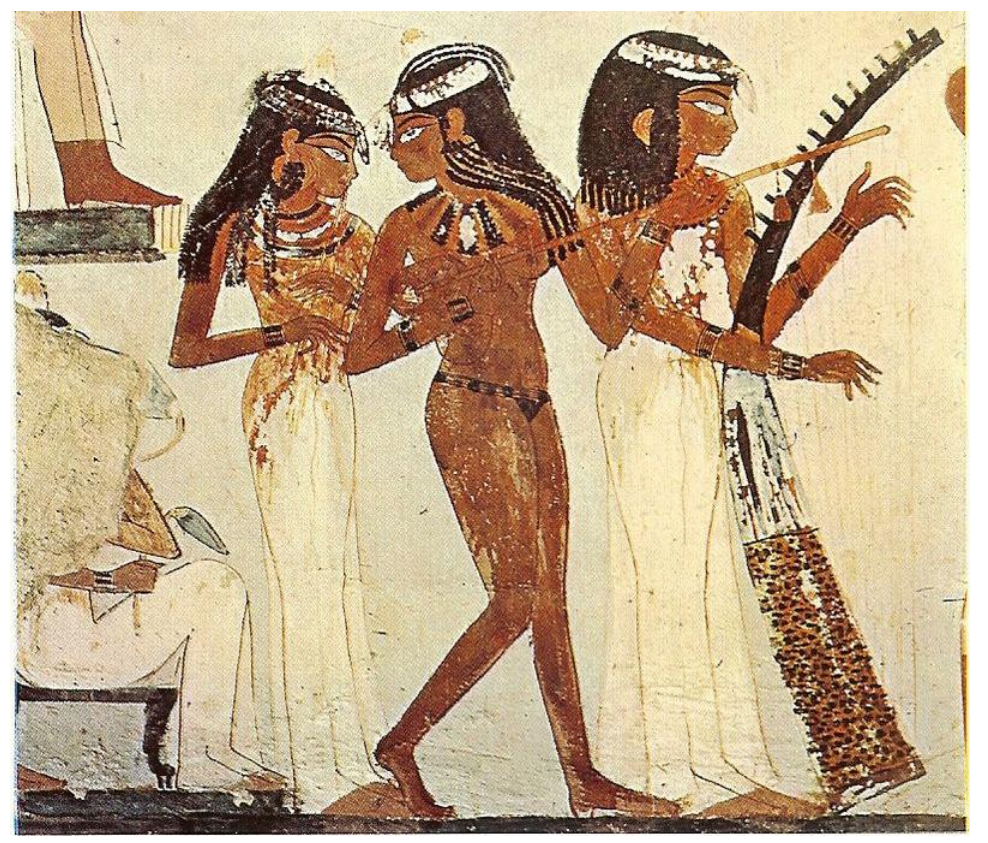

Figure 8.6: Three musicians, from the Tomb of Nakht ${ }^{121}$

Musical performance and song is linked with agricultural and work methods. The farming calendar comprised three crops per year, with music present at both sowing and harvest. Call-and-answer work songs were sung by workers in the fields, and sometimes a flautist would accompany the singers (Healey 1994). One example is the shepherd song discussed by many Egyptologists, translated from hieroglyphs:

[Call] Where is the shepherd, the shepherd of the West?

[Answer] The shepherd is in the water with the fish (Manniche 1991, p. 17.)

${ }^{121}$ This image is in the public domain. Source= https://commons.wikimedia.org/wiki/File:Tomb_of_Nakht_- three_musicians.jpg. Accessed 20/08/2016. 


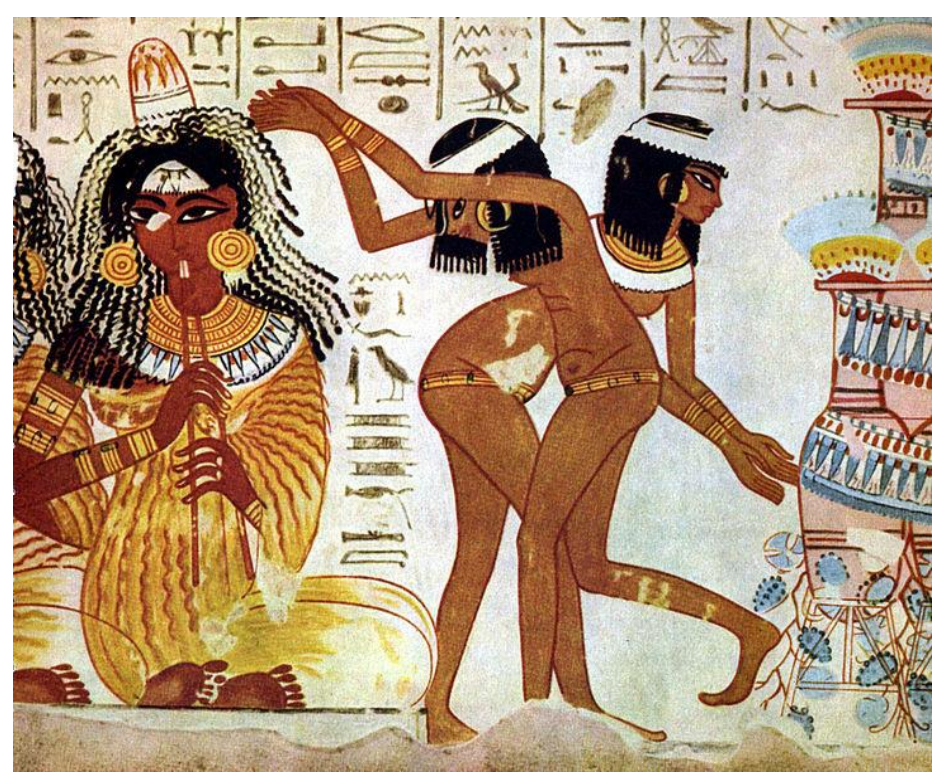

Figure 8.7: Musicians and dancers, from the Tomb of Nebamun ${ }^{122}$

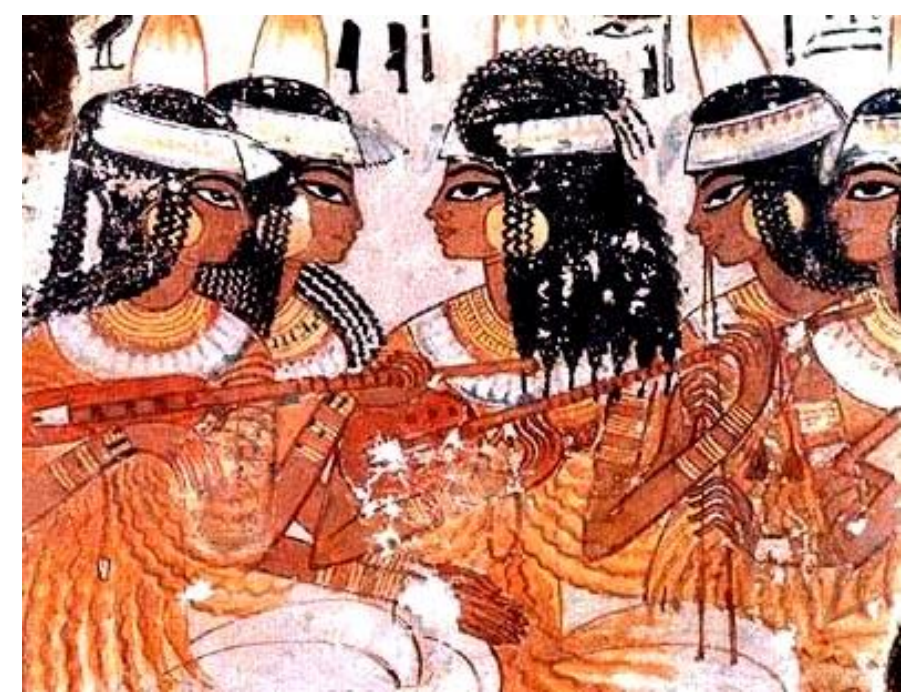

Figure 8.8: Egyptian lute players, from the Tomb of Nebamun ${ }^{123}$

\footnotetext{
${ }^{122}$ This image is in the public domain. Source=

https://commons.wikimedia.org/wiki/File:Musicians_and_dancers_on_fresco_at_Tomb_of_Nebamun .jpg. Accessed 20/08/2016.

${ }^{123}$ This image is in the public domain. Source=

https://commons.wikimedia.org/wiki/File:Egyptian lute_players_001.jpg. Accessed 20/08/2016.
} 
Other relics show flautists performing in the fields at barley harvest, and pairs of stickbeaters performing at grape harvest. Relics reveal a "pressing the grapes" dance that one must assume was accompanied by music or singing. A fragment tells of fisherman songs, another of music accompanying oarsmen, presumably to encourage rhythmic rowing as well as keep up spirits (Manniche 1991).

Alongside religion, history, mathematics, astronomy and medicine, musical theory was a standard component of the education of higher-class Egyptian elites (Healey 1994). Music was also pervasively linked with (i) ritual, (ii) daily activities of priests and other acolytes, religious scenes, and tales of the activities of the gods, (iii) court activities, such as performances for kings and other elite-class nobles, (iv) military activities (particularly drums and trumpets), ( $v$ ) funeral rites and death dances, and (vi) with conceptions of-and practices pertaining to—sexuality, fertility, and gender (for full review, see Manniche 1991). One well-discussed, poignant ancient Egyptian funeral song, apparently accompanied by the harp, is translated as proclaiming 'Let music and song be before you. Leave behind all evil and think only of joy until the day comes when we journey to that land that loves silence' (Malm 1967, p. 59).

Skilled musicians were requested to perform at significant events, to accompany dance and ritual events, and to entertain the nobles and those of higher class. Minstrels and bards brought life through musical performance to myths and legends, histories, genealogies, and spread news and stories from place to place.

Ancient Egypt was not alone in the musical revolution. Archaeologists have unearthed lyres and harps from Ancient Mesopotamia (present-day Iraq) that date back to 3,000 BCE. For one example, highly decorated lyres were found in the Royal Cemetery at Ur, dated to around 2,300 BCE (Renfrew and Bahn 2012). From 2,600 BCE, cuneiform tablets emerge that detail and list musical instruments, and give some instruction on how to play them, perhaps providing the earliest musical notation (Goodall 2013). There are dancing girl statuettes made from bronze, and seals that depict stringed musical instruments from Ancient India, that date to around 2,500 BCE. The Indian goddess Saraswati, found in almost all Ancient Indian lore from 1,000 BCE onwards, is always depicted holding a 
veena - a plucked string instrument that recorded history dates back to around 1,500 BCE (Gupta 2014)—symbolising learning and knowledge, giving rise to harmony (Kinsley 1988).

Surviving written texts from the first century of the Common Era allude to the complexity of Indian musical antiquity, its system of räga and music-theoretic framework of twenty-two microtones still in place today (although presumably in a further developed form) (Sachs 1943). There is some speculation that the oldest Indian art music-Vedic hymns-is over four thousand years old (Malm 1967), but the extent to which this hymn system might have been fully participatory as music-ritual in its earliest incarnations remains unclear.

Following the advent of the Bronze Age (1,300 BCE), bronze lurs (long, curved trumpets played by embouchure and overblowing rather than with finger holes or keys to change pitch) appear in the record. Some are depicted in cave paintings in Sweden from $1,000 \mathrm{BCE}$, and specimens from roughly the same time have been unearthed in Denmark (Goodall 2013). Bronze Age horns resembling lurs have also been discovered in Ireland (Coles 1963).

Recorded history dates the Chinese stringed zither (a predecessor of the modernday guqin and guzheng) to around 1,000 BCE (Wu 1980). Tombs have revealed zithers dating back to around $500 \mathrm{BCE}$-as well as bamboo wind instruments, bronze gong chimes, turtleshell shakers, and drums. Although according to Chinese lore the zither's origins are closer to 3,000 BCE (see e.g., Wu 1979; Gaywood 1996).

The three-tiered, chromatic-scale set of sixty-five bronze gong chimes dated to around $500 \mathrm{BCE}$ astounded the archaeological and ethnomusicological worlds when it was discovered in the 1970s (see Figure 8.9). Held together in the central chamber of the Marquis Yi of Zeng's tomb, the largest of the gong chimes weighs over $200 \mathrm{~kg}$ (Wu 1979; DeWoskin 1998). 


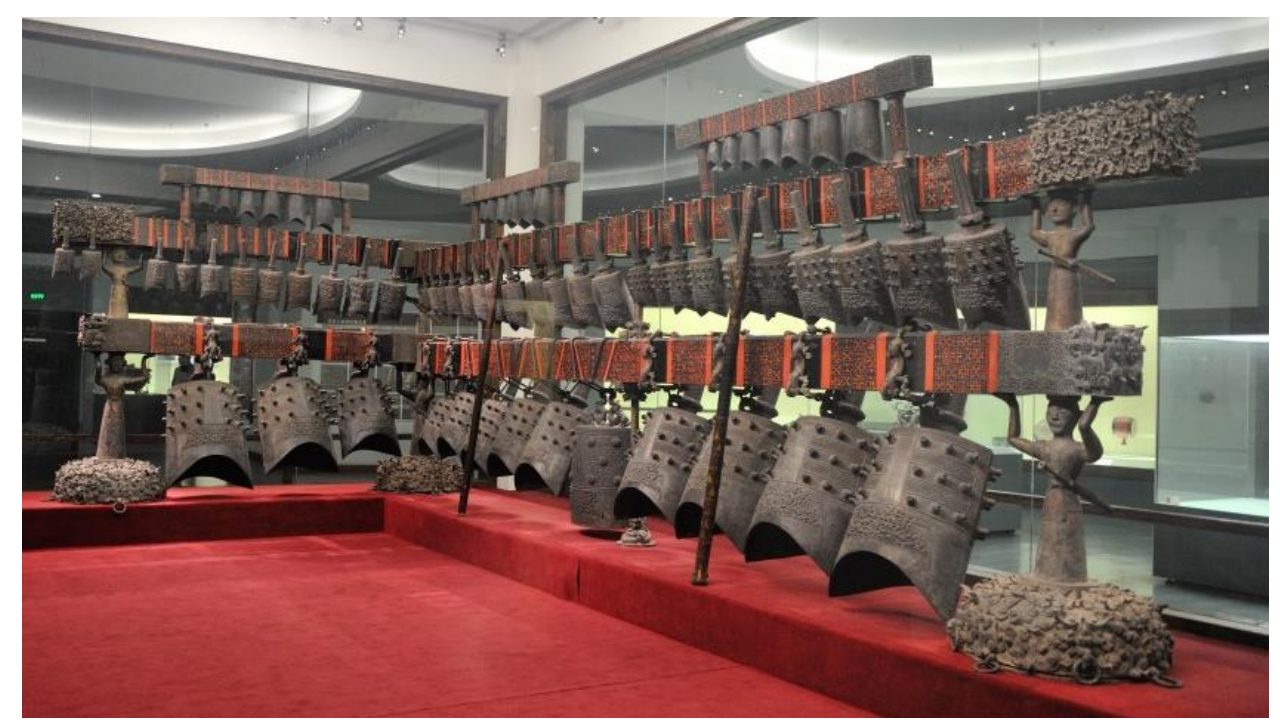

Figure 8.9: Bronze chime-bells of King Yi's tomb ${ }^{124}$

The Ancient Greeks and Romans have left much evidence of a pervasive, well-developed, fully musical culture (e.g., Bundrick 2005), including orchestras, choruses, and soloist music. Lutes, lyres, harp, and so on, feature, with items branching off into more distinct sub-kinds (West 1992). For example, West distinguishes Ancient Greek box lyres from bowl lyres, and further distinguishes both of these from "unknown type" lyres. Aulos, a two-tubed reed wind-instrument (a kind of “double oboe”) appears. Music accompanied dramas, feasts, private ceremonies, and public gatherings and festivals. It was employed in healing and education, and was offered as psalms/prayers/hymns. Domestic/personal music-making became a popular activity: since women were crudely discouraged from practicing reason (logos) in Ancient Greece, music-making became an avenue for female self-expression, creativity, and the communication of ideas and cultural values (Snyder 1998; Buckley 1998).

\footnotetext{
${ }^{124}$ Photo by "Siyuwj", freely distributable and adaptable under Creative Commons Attribution-Share Alike 4.0 International license. Source= https://commons.wikimedia.org/wiki/File:\%E6\%9B\%BE\%E4\%BE\%AF\%E4\%B9\%99\%E7\%BC\%96\%E9\%92\%9F\%E F\%BC\%8C2015-04-06 19.jpg. Accessed 20/08/2016.
} 
The Ancient Greeks developed a vast repertoire of music for all occasions, activities, and jobs, including songs/music for wool-working, rope-making, grinding/pounding, kneading/baking, working in the fields, harvesting grapes, marching/battle, and other activities and forms of manual labour (West 1992). 'Ancient Greek culture was permeated with music' (West 1992, p. 1).

Indeed, in Politics, Aristotle considers the importance of music in youth training, emphasising its reputation as a practice that builds character, affords amusement and leisure, and cultivates the mind (Aristotle 1959). Plato shares similar sentiments about the importance of musical training in The Republic; Plato 'urged on the guardians of his ideal state to ground the republic on music' (Sachs 1943, p. 254), and included music as an obligatory aspect of education in his ideal state. (For more on Aristotle's and Plato's perspectives about music, see Anderson 1994.)

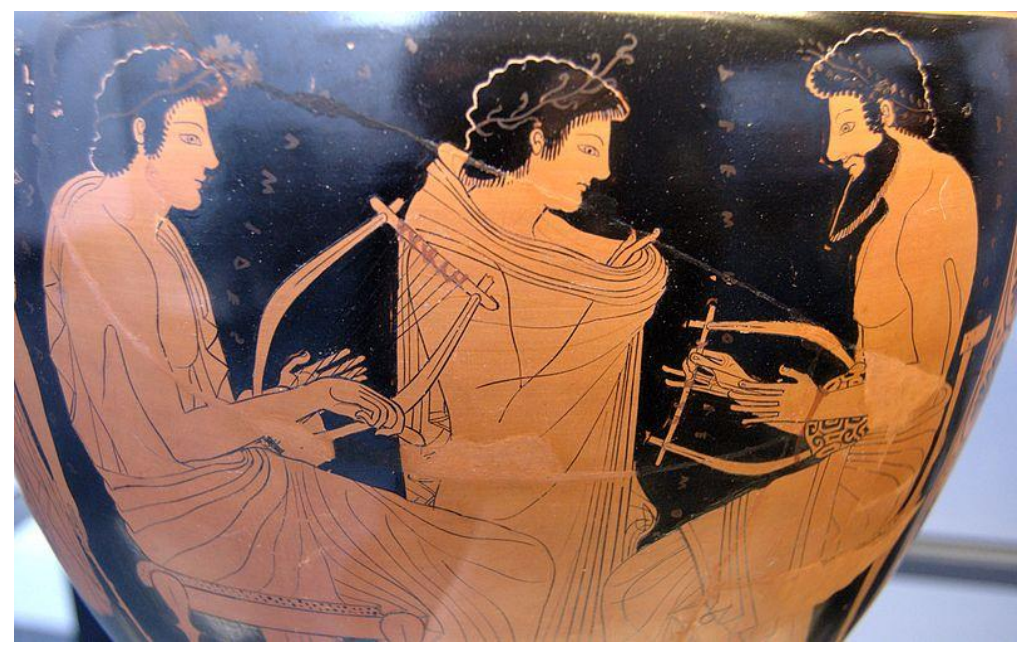

Figure 8.10: Music lesson painting on Ancient Greek vase, dated to around $510 \mathrm{BCE}^{125}$

${ }^{125}$ This image is in the public domain. Source= https://commons.wikimedia.org/wiki/File:Music_lesson_Staatliche_Antikensammlungen_2421.jpg. Accessed 20/08/2016. 


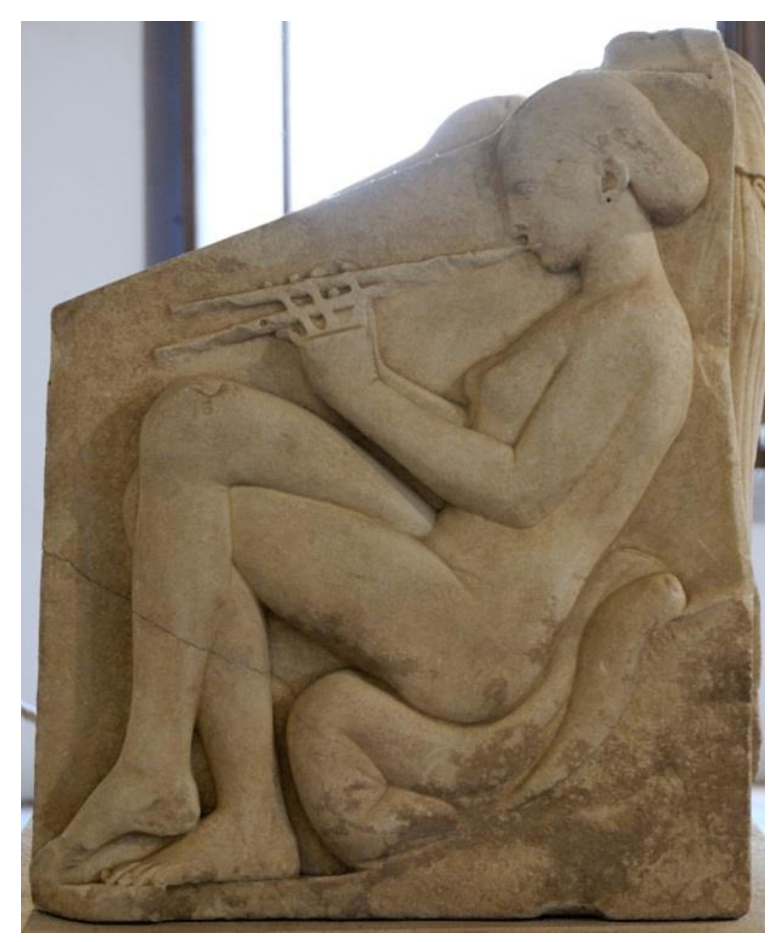

Figure 8.11: Left hand panel (Ludovisi Throne): nude playing the aulos, dated to around $460 \mathrm{BCE}^{126}$

As early as the sixth century before the Common Era, the serious music of the day was organised into "artistic" forms (nomos: professionally-performed concert pieces), on the one hand, and competitive tournament/contest pieces, on the other (Bundrick 2005). The establishment of both, as ways of making a living, would have further spurred on, through socio-cultural and socio-economic feedback, the emergence of specialist musical performers. Festivals included talent shows in which singer-songwriters would compete, winners selected by a panel of judges (Goodall 2013). The Pythian Games, a forerunner of the modern Olympics, were at first dedicated to musical and poetic sport; athletics, wrestling, and so, on appeared in these games later. There was also a not-so-serious musical culture, comprising drinking songs (skolion) and the like, knowledge of which, reportedly, was expected of everybody in Ancient Greece (Sachs 1943).

${ }^{126}$ This image is in the public domain. Source= https://commons.wikimedia.org/wiki/File:Ludovisi_throne_Altemps_Inv8570_n3.jpg. Accessed 20/08/2016. 
The Ancient Greeks developed the first organs around 250 BCE, which used water tanks to pressurise the air for the organ pipes. Figure 8.12 depicts two musicians, including an organ player, providing the music at a Gladiator match:

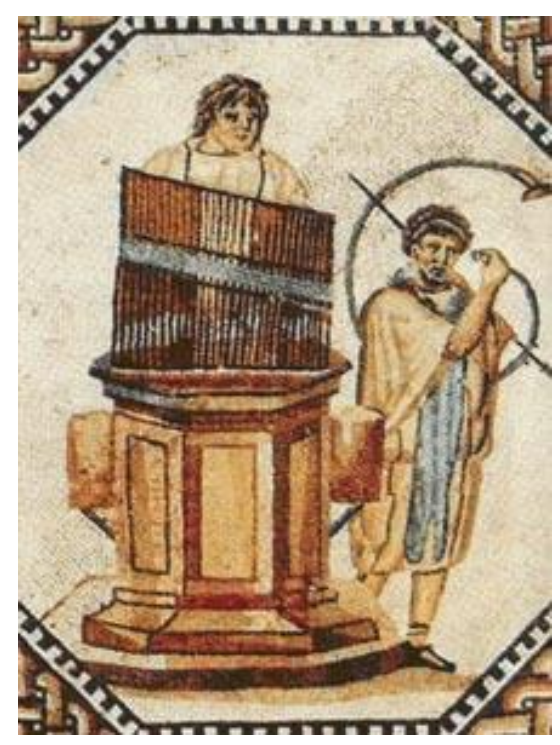

Figure 8.12: Hydraulis (water organ) and bukanē (curved trumpet) musicians ${ }^{127}$

So here we have arrived at the beginnings of (written) music philosophy, history and theory (for more detailed surveys, see Hagel 2010; West 1992; Anderson 1994; Bundrick 2005). Clearly music was just as ubiquitous then as it is now, despite a lack of recording technology or industry. The Ancient Egyptian, Greek, and Roman worlds are often seen as the beginning of Western civilisation and it is clear that music was a key ingredient in the lives of the people inhabiting those worlds. Humans construct their civilisations, one way or another; ever since their beginning, these have been, universally, musical ones.

\footnotetext{
${ }^{127}$ This image is in the public domain. Source= https://commons.wikimedia.org/wiki/File:Hydraulis_001.jpg. Accessed 20/08/2016.
} 
So all over the ancient world, there were people learning music, performing music, coming up with new and innovative ways of being musical. From here the development of ancient music to that of the present day is well documented (for a popular narrative see Goodall 2013), as is the diffusion and subsequent evolution of musical principles, practices, and concepts over the globe as associated religions, ideologies and cultural groups expanded (for one example, see Malm 1967, chapter 7, on the extensive influence of Chinese music on musical systems in Korea and Japan). And although the Anglophone world may owe the word "music" to the Greeks, we have seen that music (musicking, musicality) itself has a much more ancient evolutionary history, deeply connected to hominin socio-cognitive evolution.

\section{Concluding remarks}

Modern musics in various guises, forms, and functions today undoubtedly have a complex genesis. My model of hominin evolution and my narrative of music's origins, expansion and stabilisation highlights the simple/systematic/speculative calibration of human cognition, and musicality's deeply entrenched mosaic nature. Pre-Homo simple/opportunistic communication developed into Oldowan/Acheulean intentional systematic musicality as a result of myriad dynamic factors: social, ecological and psychological pressures, bio-cultural co-evolution, and niche construction.

This built the cognitive, gestural, and ritualistic cultural platform which enabled the control of fire and the evolution of systematic/speculative proto-music and spoken protolanguage in the Late Acheulean. From 250 Kya symbolic/mythic/oral/musical cumulative culture gave our ancestors the scaffolding for developed brains that sent us inexorably on our way to becoming modern, ultra-effective niche-constructing present-day humans. Hominin niche construction gave rise to many surprising innovations, and the musics of the present day are but a set of fascinating outcomes of millions of years of bio-cultural co-evolution and socio-cognitive niche construction.

Finally, I return to conceptual and methodological reflection. First, in these final two chapters, I have conceived of music (taking a relational conception of music) not as a symbolic or necessarily artistic/aesthetic form of communicative expression, which would 
be consistent with hypotheses about the "great leap forward" cognitive revolution or creative/symbolic explosion, around 40 to 50 Kya (e.g., Klein and Edgar 2002; Klein 2013). And I do not conceive of music as encapsulated within a unitary cognitive module or as a byproduct of unitary cognitive modules (as in Santa Barbara-style Evolutionary Psychology), as a means for tunnelling between domain-specific modules (Cross 2006, 2007), or as innate, or individualist. Rather, I conceive of music as social (although, certainly capable of being individualistic), and as a kind of crane (Dennett 1995) - a patchwork of myriad features. I emphasise music's affective and playful communicative effects, the complex suite of co-adapted traits underwriting and interfacing in various ways with music (executive control, memory, theory of mind, speculative cognition). These point me to an incremental picture of music evolution, and a role for aspects of the social brain hypothesis. Thus this evolutionary scenario, in part, contributes to the broad research agenda of Gamble, Gowlett, and Dunbar that is manifest in their collaborative British Academy Centenary Research Project. It is also a contribution to ecological, socio-cognitive and cultural niche construction modelling of hominin evolution generally.

Second, I have not merely argued for a narrative comprising first-order facts, interpretations, and educated empirical guesses, and I have not merely stressed the role of sociality, culture and niche construction in human cognitive and technological co-evolution, and the importance of taking gene-culture co-evolution seriously in evolutionary hypotheses of music (or other aspects of "human nature") (contra, for example, Miller 2001). I have a methodological argument here too: I have proposed, and given an example of, a lineage explanation, requiring integration of streams of evidence from multiple research agendas, notably the palaeoanthropological and archaeological records. In contrast to the hypotheses couched squarely within the adaptation/by-product framework, my account seeks not a specific proper function, nor a unitary signature, of music. It integrates aspects relating to ecological niche, socio-cognitive co-evolution and anatomic/morphological evolution, social transmission, technologies, demographics and group size, socio-economic organisation, and cultural complexities. While we do not have direct evidence of Late Acheulean proto-music, for instance, we can probe this integrated framework in order to shed light on the communicative and affective demands on Pleistocene hominins. New research in the evolution of the emotions, as I have noted, may provide important avenues 
for testing hypotheses about these connections. And while new material evidence and use-wear/experimental analysis might undermine or falsify some of my conjectures, or require re-dating some aspects of my timeline, it would not destabilise the methodological framework that I have been both advocating and utilising-conceiving the evolution of musicality/music 'as a special case of a general process, one whose operations we can more directly identify' (Sterelny 2016b, p. 184). This methodology, I suspect, will prove to be a fuel for explanatory success. 


\section{Conclusion}

In this thesis I have developed a naturalistic framework for theorising about music. In accordance with my philosophical naturalism (outlined in chapter 1), I have motivated and defended a polysemy analysis of "sound" (chapter 2), and conceptual pluralism about music (chapter 3). I have demonstrated the utility of a pluralistic perspective in the scientific analysis of music in general (chapter 4). And turning to metaphysics research, I have focused on one particular concept - the concept of the Western musical artwork, a key concept in the analytic metaphysics of music —and defended a naturalistically-respectable fictionalist approach to the ontological category specified in practice by that concept (chapter 5). I have critiqued the adaptation/by-product framework that has largely influenced academic debate about the evolution of music, and I have defended a co-evolutionary, niche construction approach to music (chapter 6). I then put this perspective to work. I have developed a model of early hominin evolution and emphasised the place of musicality, conceived as a mosaic of features, in the general picture (chapter 7) and I have developed a narrative of the evolution of music throughout the latter stages of hominin evolution (chapter 8 ), pointing to areas for future research and reflecting conceptually and methodologically on evolution of music research. 


\section{References}

Abrahamsen, Adele (1987) "Bridging boundaries versus breaking boundaries—psycholinguistics in perspective", Synthese 72(3): 355-388.

Adler, Daniel (2009) “The earliest musical tradition”, Nature 460: 695-696.

Aich, H., R. Moos-Heilen and E. Zimmermann (1990) "Vocalizations of adult gelada baboons (Theropithecus gelada): acoustic structure and behavioural context”, Folia Primatologica 55: 109-132.

Alemseged, Zeresenay, Fred Spoor, William Kimbel, René Bobe, Denis Geraads, Denné Reed and Jonathan Wynn (2006) "A juvenile early hominin skeleton from Dikika, Ethiopia”, Nature 443: 296-301.

Anderson, R. J., P. Carrick, G. J. Levitt, A. Share (1997) "Holdfasts of adult kelp Ecklonia maxima provide refuges from grazing for recruitment of juvenile kelps", Marine Ecology Progress Series 159: 265-273.

Anderson, Warren (1994) Music and musicians in Ancient Greece (Ithaca: Cornell University Press).

Antón, Susan, Richard Potts and Leslie Aiello (2014) “Evolution of early Homo: an integrated biological perspective" Science 345(6192): 1236828.

Antón, Susan and J. Josh Snodgrass (2012) "Origins and evolution of genus Homo: new perspectives”, Current Anthropology 53(suppl-6): s479-496.

Arbib, Michael (2013) "Five terms in search of a synthesis", in M. A. Arbib (ed), Language, music and the brain: a mysterious relationship (Cambridge: MIT Press), pp. 3-44.

Ariew, André (2003) “Ernst Mayr's ‘ultimate/proximate' distinction reconsidered and reconstructed”, Biology and Philosophy 18(4): 553-565.

Ariew, André (2007) “Innateness", in M. Matthen, C. Stephens (eds), Philosophy of biology (Amsterdam: Elsevier), pp. 567-584.

Aristotle (1959) Politics (Britain: J.M. Dent \& Sons).

Armstrong, David (1968) A materialist theory of the mind (London: Routledge).

Ashley, Gail, Doris Barboni, Manuel Domínguez-Rodrigo, Henry Bunn, Audax Mabulla, Fernando DiezMartin, Rebeca Barba and Enrique Baquedano (2010) "A spring and wooded habitat at FLK Zinj and their relevance to origins of human behavior", Quaternary Research 74(3): 304-314.

Attwell, Laura, Kris Kovarovic and Jeremy Kendal (2015) "Fire in the Plio-Pleistocene: the functions of hominin fire use, and the mechanistic, developmental and evolutionary consequences", Journal of Anthropological Sciences 93: 1-20.

Ayoub, Chakib, Laudi Rizk, Chadi Yaacoub, Dorothy Gaal and Zeev Kain (2005) "Music and ambient operating room noise in patients undergoing spinal anesthesia", Anesthesia and Analgesia 100(5): 1316-1319.

Bailey, Martin (2013) “Ice Age Lion Man is world's earliest figurative sculpture", The Art Newspaper, URL= <http://www.theartnewspaper.com/articles/Ice-Age-iLion-Mani-is-worlds-earliest-figurativesculpture/28595/>. Accessed 10 August 2014. 
Balaguer, Mark (2009) "Fictionalism, theft, and the story of mathematics", Philosophica Mathematica 17(2): 131-162.

Ball, Philip (2010) The music instinct (London: Vintage).

Balter, Michael (2015) “World's oldest stone tools discovered in Kenya”, Science doi. 10.1126/science.aab2487.

Barac, Victor (1999) "From primitive to pop: foraging and post-foraging hunter-gatherer music", in R. Lee, R. Daly (eds), Cambridge encyclopedia of hunters and gatherers (Cambridge: Cambridge University Press), pp. 434-440.

Barham, Lawrence (2013) From hand to handle: the first industrial revolution (Oxford: Oxford University Press).

Barkow, Jerome, Leda Cosmides and John Tooby (1992) The adapted mind: evolutionary psychology and the generation of culture (New York: Oxford University Press).

Barrow, John (2005) The artful universe expanded (Oxford: Oxford University Press).

Bateson, Patrick (2017) "Play and evolution", in R Joyce (ed), Routledge companion to evolution and philosophy (Oxon: Routledge).

Bateson, Patrick and Paul Martin (2013) Play, playfulness, creativity and innovation (Cambridge: Cambridge University Press).

Beatty, John (1994) “The proximate/ultimate distinction in the multiple careers of Ernst Mayr", Biology and Philosophy 9(3):333-356.

Beban, Daniel (2001) Going for a song: the New Zealand chant of auctions, housie and racing. Master's Thesis, Victoria University of Wellington.

Beja-Pereira, Albano, Gordon Luikart, Philip England, Daniel Bradley, Oliver Jann, Giorgio Bertorelle, Andrew Chamberlain, Telmo Nunes, Stoitcho Metodiev, Nuno Ferrand and Georg Erhard (2003) "Gene-culture coevolution between cattle milk protein genes and human lactase genes", Nature Genetics 35: 311-313.

Bello, Juan, Robert Rowe, Carlos Guedes and Godfried Toussaint (2015) "Five perspectives on musical rhythm", Journal of New Music Research 44(1): 1-2.

Belfi, Amy, Brett Karlan and Daniel Tranel (2015) “Music evokes vivid autobiographical memories", Memory 24(7): 979-989.

Bender, Andrea, Edwin Hutchins and Douglas Medin (2010) "Anthropology in cognitive science", Topics in Cognitive Science 2(3): 374-385.

Bender, Renato, Phillip Tobias and Nicole Bender (2012) "The savannah hypotheses: origin, reception and impact on palaeoanthropology", History and Philosophy of the Life Sciences 34: 147-184.

Bentham, Jeremy (1932) Bentham's theory of fictions [C. K. Ogden, ed.] (London: Kegan Paul, Trench, Trubner and Co. Ltd.).

Berthelet, A. and J. Chavaillon (2001) "The early Palaeolithic butchery site of Barogali (Republic of Djibouti)", in G. Cavarretta, P. Gioia, M. Mussi, M. R. Palombo (eds), The World of Elephants: International Congress Proceedings (Rome: Consiglio Nazionale delle Ricerche), pp. 176-179. 
Bohlman, Philip (1999) “Ontologies of music”, in N. Cook, M. Everist (eds), Rethinking music (Oxford: Oxford University Press), pp. 17-34.

Borjon, Jeremy and Asif Ghazanfar (2014) "Convergent evolution of vocal cooperation without convergent evolution of brain size", Brain, Behavior and Evolution 84: 93-102.

Bowra, C. M. (1962) Primitive song (London: Weidenfeld and Nicolson).

Braddon-Mitchell, David and Robert Nola (2009) “Introducing the Canberra plan”, in D. BraddonMitchell, R. Nola (eds), Conceptual analysis and philosophical naturalism (Cambridge: MIT Press), pp. 1-20.

Braun, David, John Harris, Naomi Levin, Jack McCoy, Andy Herries, Marion Bamford, Laura Bishop, Brian Richmond and Mzalendo Kibunjia (2010) "Early hominin diet included diverse terrestrial and aquatic animals 1.95 Ma in East Turkana, Kenya", Proceedings of the National Academy of Sciences USA 107: 10002-10007.

Brigandt, Ingo (2002) "Homology and the origin of correspondence", Biology and Philosophy 17: 389-407.

Brigandt, Ingo (2003a) "Homology in comparative, molecular and evolutionary biology: the radiation of a concept", Journal of Experimental Zoology (Molecular and Developmental Edition) 299B: 9-17.

Brigandt, Ingo (2003b) “Species pluralism does not imply species eliminativism”, Philosophy of Science 70(5): 1305-1316.

Brigandt, Ingo (2010) "Beyond reduction and pluralism: toward an epistemology of explanatory integration in biology", Erkenntnis 73(3): 295-311.

Briggs, Jean (2000) "Conflict management in a modern Inuit community", in P. P. Schweitzar, M. Biesele, R. K. Hitchcock (eds), Hunters and gatherers in the modern world: conflict, resistance, and selfdetermination (New York: Berghahn), pp. 110-124.

Broadhurst, C. Leigh, Stephen Cunnane and Michael Crawford (1998) "Rift Valley lake fish and shellfish provided brain-specific nutrition for early Homo", British Journal of Nutrition 79(1): 3-21.

Brock, Stuart (2002) “Fictionalism about fictional characters”, Noûs 36(1): 1-21.

Brock, Stuart (2015) “Fictionalism, fictional characters, and fictionalist inference”, in S. Brock, A. Everett (eds), Fictional objects (Oxford: Oxford University Press), pp. 230-254.

Brock, Stuart (2016) “Fictionalism about fictional characters revisited”, Res Philosophica 93(2): 377-403.

Brown, Steven (2000) "Evolutionary models of music: from sexual selection to group selection", in F. Tonneau, N. Thompson (eds), Perspectives in ecology 13: behavior, evolution and culture (New York: Plenum), pp. 231-281.

Brown, Steven, Patrick Savage, Albert Min-Shan Ko, Mark Stoneking, Ying-Chin Ko, Jun-Hun Loo and Jean A. Trejaut (2013) "Correlations in the population structure of music, genes and language", Proceedings of the Royal Society B: Biological Sciences 281: 20132072.

Brusse, Carl (2016) "Planets, pluralism, and conceptual lineage”, Studies in History and Philosophy of Modern Physics 53: 93-106. 
Bryant, Gregory and H. Clark Barrett (2007) "Recognizing intentions in infant-directed speech: evidence for universals", Psychological Science 18(8): 746-751.

Buckley, Ann (1998) “Organised sound and tonal art in long-term perspective”, in A. Buckley (ed), Hearing the past: essays in historical ethnomusicology and the archaeology of sound (Liège: Université de Liège), pp. 9-16.

Bulbulia, Joseph (2005) "Are there any religions? An evolutionary explanation", Method and Theory in the Study of Religion 17(2): 71-100.

Bullot, Nicolas and Paul Égré (eds) (2010a) Review of Philosophy and Psychology 1(1): 1-156.

Bullot, Nicolas and Paul Égré (2010b) “Editorial: objects and sound perception”, Review of Philosophy and Psychology 1(1): 5-17.

Bundrick, Sheramy (2005) Music and image in Classical Athens (New York: Cambridge University Press).

Bunn, Henry and Alia Gurtov (2013) "Prey mortality profiles indicate that Early Pleistocene Homo at Olduvai was an ambush predator”, Quaternary International 322/323: 44-53.

Bunn, Henry and Travis Rayne Pickering (2010) "Bovid mortality profiles in paleoecological context falsify hypotheses of endurance running-hunting and passive scavenging by early Pleistocene hominins", Quaternary Research 74(3): 395-404.

Burgess, John (2004) “Mathematics and Bleak house”, Philosophia Mathematica 12(1): 18-36.

Burgess, John and Gideon Rosen (1997) A subject with no object (Oxford: Clarendon).

Burkart, Judith, Sarah Hrdy and Carel van Schaik (2009) "Cooperative breeding and human cognitive evolution", Evolutionary Anthropology 18(5): 175-186.

Burling, Robbins (2007) The talking ape: how language evolved (New York: Oxford).

Buss, David (2005) The handbook of evolutionary psychology (New Jersey: Wiley).

Calcott, Brett (2009) "Lineage explanations: explaining how biological mechanisms change", British Journal for the Philosophy of Science 60(1): 51-78.

Calcott, Brett (2013) "Why the proximate-ultimate distinction is misleading, and why it matters for understanding the evolution of cooperation", in K. Sterelny, R. Joyce, B. Calcott, B. Fraser (eds), Cooperation and its evolution (Massachusetts: MIT Press), pp. 249-264.

Call, Josep and Michael Tomasello (2008) "Does the chimpanzee have a theory of mind? 30 years later", Trends in Cognitve Science 12(5): 187-192.

Camp, Charles and Bruno Nettl (1955) “The musical bow in Southern Africa”, Anthropos 50: 65-80.

Caplan, Ben (2004) "Creatures of fiction, myth, and imagination", American Philosophical Quarterly 41(4): 331-337.

Caplan, Ben and Carl Matheson (2006) "Defending musical perdurantism”, British Journal of Aesthetics 46(1): 59-69.

Carroll, Joseph (1998) "Steven Pinker's cheesecake for the mind", Philosophy and Literature 22(2): 478-485. 
Carvalho, Susana, Eugénia Cunha, Cláudia Sousa and Tetsuro Matsuzawa (2008) “Chaînes opératoires and resource-exploitation strategies in chimpanzee (Pan troglodytes) nut cracking", Journal of Human Evolution 55: 148-163.

Casati, Roberto and Jérôme Dokic (1994) La philosophie du son (Nîmes: Chambon).

Casati, Roberto and Jérôme Dokic (2009) "Some varieties of spatial hearing”, in M. Nudds, C. O'Callaghan (eds), Sounds and perceptions: new philosophical essays (New York: Oxford University Press), pp. 97-110.

Casati, Roberto and Jérôme Dokic (2012) "Sounds", in E. Zalta (ed), Stanford encyclopedia of philosophy (Winter 2012 edition), URL= <http://plato.stanford.edu/archives/win2012/entries/sounds/>. Accessed 14 March 2013.

Cassoli, Pier and Antonio Tagliacozzo (1997) "Butchering and cooking of birds in the Palaeolithic site of Grotta Romanelli (Italy)", International Journal of Osteoarchaeology 7(4): 303-320.

Chalmers, David (2011) “Verbal disputes”, Philosophical Review 120(4): 515-566.

Charlton, Benjamin, Piera Filippi and W. Tecumseh Fitch (2012) "Do women prefer more complex music around ovulation?", PLoS One 7(4): e35626.

Chase, Philip and Harold Dibble (1987) "Middle Paleolithic symbolism: a review of current evidence and interpretations", Journal of Anthropological Archaeology 6: 263-296.

Clarke, Esther, Ulrich Reichard and Klaus Zuberbühler (2006) “The syntax and meaning of wild gibbon songs", PLoS One 1(1): e73.

Clay, Zanna and Klaus Zuberbühler (2011) "Bonobos extract meaning from call sequences", PLoS One 6(4): e18786.

Clay, Zanna and Klaus Zuberbühler (2013) "Food-associated calling sequences in bonobos", Animal Behaviour 77(6): 1387-1396.

Coles, John (1963) "Irish Bronze Age horns and their relations with Nothern Europe", Proceedings of the Prehistoric Society 29: 326-356.

Colleran, Heidi and Ruth Mace (2011) "Contrasts and conflicts in anthropology and archaeology: the evolutionary/interpretive dichotomy in human behavioural research", in E. Cochrane, A. Gardner (eds), Evolutionary and interpretive archaeologies: a dialogue (California: Left Coast Press), pp. 281-305.

Collingwood, R. G. (1938) The principles of art (Oxford: Oxford University Press).

Colyvan, Mark (2015) "Indispensability arguments in the philosophy of mathematics" in E. Zalta (ed), Stanford encyclopedia of philosophy (Spring 2015 edition), URL=

<http://plato.stanford.edu/archives/spr2015/entries/mathphil-indis/>. Accessed 28 August 2016.

Colyvan, Mark, Stefan Linquist, William Grey, Paul Griffiths, Jay Odenbaugh and Hugh Possingham (2009) "Philosophical issues in ecology: recent trends and future directions", Ecology and Society 14: 22.

Conard, Nicholas (2009) "A female figurine from the Basal Aurignacian of Hohle Fels Cave in southwestern Germany”, Nature 459: 248-252. 
Conard, Nicholas and Maria Malina (2008) "New evidence for the origins of music from the caves of the Swabian Jura", in A. A. Both, R. Eichmann, E. Hickmann, L.-Ch. Koch (eds), Orient-Archäologie Band 22. Studien zur Musikarchäologie VI (Rahden: Verlag Marie Leidorf GmbH), pp. 13-22.

Conard, Nicholas, Maria Malina and Susanne Münzel (2009) "New flutes document the earliest musical tradition in southwestern Germany", Nature 460: 737-740.

Cook, Peter, Andrew Rouse, Margaret Wilson and Colleen Reichmuth (2013) “A California sea lion (Zalophus californianus) can keep the beat: motor entrainment to rhythmic auditory stimuli in a non vocal mimic", Journal of Comparative Psychology 127(4): 412-427.

Cowlishaw, Guy (1996) "Sexual selection and information content in gibbon song bouts", Ethology 102(2): 272-284.

Cox, Renée (1986) “A defence of musical idealism”, British Journal of Aesthetics 26(2): 133-142.

Crane, Tim (2014) Objects of thought (Oxford: Oxford University Press).

Craver, Carl (2005) "Beyond reduction: mechanisms, multifield integration and the unity of neuroscience", Studies in the History and Philosophy of Biological and Biomedical Sciences 36: 373-395.

Craver, Carl (2007) Explaining the brain: mechanisms and the mosaic unity of neuroscience (Oxford: Oxford University Press).

Crockford, Catherine, Roman Wittig, Roger Mundy and Klaus Zuberbühler (2012) “Wild chimpanzees inform ignorant group members of danger", Current Biology 22(2): 142-146.

Crompton, Robin, Todd Pataky, Russell Savage, Kristiaan D’Août, Matthew Bennett, Michael Day, Karl Bates, Sarita Morse and William Sellers (2012) "Human-like external function of the foot, and fully upright gait, confirmed in the 3.66 million year old Laetoli hominin footprints by topographic statistics, experimental footprint-formation and computer simulation", Journal of the Royal Society Interface 9(69): 707-719.

Cross, Ian (2006) “Music and social being”, Musicology Australia 28: 114-126.

Cross, lan (2007) "Music and cognitive evolution", in L. Barret and R. Dunbar (eds), Oxford handbook of evolutionary psychology (Oxford: Oxford University Press), pp. 649-667.

Cross, lan (2012a) "Music as a social and cognitive process", in P. Rebuschat, M. Rohrmeier, J. A. Hawkins, I. Cross (eds), Language and music as cognitive systems (Oxford: Oxford University Press), pp. 315-328.

Cross, lan (2012b) "Cognitive science and the cultural nature of music", Topics in Cognitive Science 4: 668-677.

Cross, lan (2012c) “Music and biocultural evolution", in M. Clayton, T. Herbert, R. Middleton (eds), The cultural study of music: a critical introduction (London: Routledge), pp. 15-27.

Cross, lan (2016) "The nature of music and its evolution", in S. Hallam, I. Cross, M. Thaut (eds), Oxford handbook of music psychology, second edition (Oxford: Oxford University Press), pp. 3-17.

Cross, lan, Ezra Zubrow and Frank Cowan (2002) "Musical behaviours and the archaeological record: a preliminary study", Experimental archaeology: British archaeological reports in international series 1035: 25-34. 
Cuenca-Solana, David, F. Igor Gutiérrez-Zugasti, Manuel R. González-Morales, Jesus SetiénMarquineaz, Estela Ruiz-Martinez, Alejandro García-Moreno and Ignacio Clemente-Conte (2013) "Shell technology, rock art, and the role of marine resources during the Upper Paleolithic", Current Anthropology 54(3): 370-380.

Currie, Adrian (2013) "Convergence as evidence”, British Journal for Philosophy of Science 64(4): 763-786.

Currie, Adrian (2015) "Marsupial lions and methodological omnivory: function, success and reconstruction in paleobiology", Biology and Philosophy 30(2): 187-209.

Currie, Adrian (2016a) "The mystery of the Triceratops's mother: how to be a realist about the species category", Erkenntnis 81(4): 795-816.

Currie, Adrian (2016b) "Hot-blooded gluttons: dependency, coherence, and method in the historical sciences", British Journal for the Philosophy of Science, doi. 10.1093/bjps/axw005.

Currie, Adrian and Anton Killin (2016) "Musical pluralism and the science of music", European Journal for Philosophy of Science 6(1): 9-30.

Currie, Gregory (1989) An ontology of art (New York: St. Martin's Press).

Currie, Gregory (1990) The nature of fiction (Cambridge: Cambridge University Press).

Daly, Chris (2008) “Fictionalism and the attitudes", Philosophical Studies 139(3): 423-440.

Darwin, Charles (1871) The descent of man, and selection in relation to sex (New York: Appleton \& Co.).

Davies, David (2004) Art as performance (Massachusetts: Blackwell).

Davies, David (2009) "Works and performances in the performing arts", Philosophy Compass 4(5): 744-755.

Davies, E. Harold (1947) “Music in primitive society”, Anthropological Society of South Australia Occasional Publication No. 2 (Adelaide: University of Adelaide).

Davies, Stephen (2001) Musical works and performances: a philosophical exploration (Oxford: Oxford University Press).

Davies, Stephen (2003) Themes in the philosophy of music (New York: Oxford University Press).

Davies, Stephen (2004) “The cluster theory of art”, British Journal of Aesthetics 44: 297-300.

Davies, Stephen (2009) "Review of Aesthetics and music", Analysis 69: 397-398.

Davies, Stephen (2012a) “On defining music”, Monist 95: 535-555.

Davies, Stephen (2012b) The artful species: aesthetics, art and evolution (Oxford: Oxford University Press).

Davies, Stephen (2017) “Evolution of music", in N. Nielson, J. Levinson, T. McAuley (eds), Oxford handbook of music and philosophy (Oxford: Oxford University Press).

Davis, M. (1994) “Folk music psychology”, Psychologist 7: 537. 
Dawes, Gregory and James Maclaurin (2013) "What is religion?: identifying the explanandum", in G. Dawes, J. Maclaurin (eds), A new science of religion (New York: Routledge), pp. 10-25.

Debus, Dorothea (2014) “'Mental time travel': remembering the past, imagining the future, and the particularity of events", Review of Philosophy and Psychology 5(3): 333-350.

Dennett, Daniel (1995) Darwin's dangerous idea (New York: Simon \& Schuster).

D’Errico, Francesco, Christopher Henshilwood, Graeme Lawson, Marian Vanhaeren, Anne-Marie Tillier, Marie Soressi, Frédérique Bresson, Bruno Maureille, April Nowell, Joseba Lakarra, Lucinda Backwell and Michèle Julien (2003) "Archaeological evidence for the emergence of language, symbolism, and music —an interdisciplinary perspective", Journal of World Prehistory 17(1): 1-70.

D'Errico, Francesco and Chris Stringer (2011) "Evolution, revolution or saltation scenario for the emergence of modern cultures?", Philosophical Transactions of the Royal Society B: Biological Sciences 366: 1060-1069.

Deutsch, Diana (1984) "Psychology and music", in M. H. Bornstein (ed), Psychology and its allied disciplines (Hillsdale: Erlbaum), pp. 155-194.

Deutsch, Harry (2000) “Making up stories", in A. Everett, T. Hofweber (eds), Empty names, fiction and the puzzles of non-existence (Stanford: Center for the Study of Language and Information), pp. 149-181.

DeWoskin, Kenneth (1998) "Symbol and sound: reading early Chinese instruments", in A. Buckley (ed), Hearing the past: essays in historical ethnomusicology and the archaeology of sound (Liège: Université de Liège), pp. 103-122.

Diamond, Beverley (2008) Native American music in eastern North America (Oxford: Oxford University Press).

Diamond, Jared (1992) The third chimpanzee (London: Vintage).

Diedrich, Cajus (2015) “'Neanderthal bone flutes': Simply products of Ice Age spotted hyena scavenging activities on cave bear cubs in European cave bear dens", Royal Society Open Science 2: 140022 .

Dissanayake, Ellen (1982) "Aesthetic experience and human evolution", Journal of Aesthetics and Art Criticism 41(2): 145-155.

Dissanayake, Ellen (2000a) Art and intimacy: how the arts began (Seattle: University of Washington Press).

Dissanayake, Ellen (2000b) "Antecedents of the temporal arts in early mother-infant interaction", in N. Wallin, B. Merker, S. Brown (eds), Origins of music (Cambridge: MIT Press), pp. 389-410.

Dissanayake, Ellen (2008) "If music is the food of love, what about survival and reproductive success?” Musicae Scientiae 12(suppl-1): 169-195.

Dissanayake, Ellen (2009) "Root, leaf, blossom, or bole: Concerning the origin and adaptive function of music", in S. Malloch, C. Trevarthen (eds), Communicative Musicality (Oxford University Press), pp. 17-30.

Dodd, Julian (2000) “Musical works as eternal types", British Journal of Aesthetics 40(4): 424-440. 
Dodd, Julian (2002) “Defending musical Platonism”, British Journal of Aesthetics 42(4): 380-402.

Dodd, Julian (2007) Works of music: an essay in ontology (New York: Oxford University Press).

Dodd, Julian (2008) “Musical works: ontology and metaontology", Philosophy Compass 3(6): 1113-1134.

Domínguez-Rodrigo, Manuel, Travis Rayne Pickering and Henry Bunn (2012) “Experimental study of cut marks made with rocks unmodified by human flaking and its bearing on claims of $\sim 3.4$-millionyear-old butchery evidence from Dikka, Ethiopia”, Journal of Archaeological Science 39(2): 205-214.

Donald, Merlin (2001) A mind so rare: the evolution of human consciousness (New York: WW Norton \& Co.).

Downes, Stephen (2010) "Evolutionary psychology" in E. Zalta (ed), Stanford encyclopedia of philosophy (Fall 2010 edition), URL= <http://plato.stanford.edu/archives/fall2010/entries/evolutionarypsychology/>. Accessed 14 August 2014.

Duffin, Ross (2007) How equal temperament ruined harmony (and why you should care) (New York: W.W. Norton \& Co).

Dufour, Valérie, Nicholas Poulin, Charlotte Curé and Elisabeth H. M. Sterk (2015) “Chimpanzee drumming: a spontaneous performance with characteristics of human musical drumming", Scientific Reports 5: 11320.

Dunbar, Robin (1996) Grooming, gossip and the evolution of language (London: Faber \& Faber).

Dunbar, Robin (2004) The human story: a new history of mankind's evolution (London: Faber \& Faber).

Dunbar, Robin (2008) "Why humans aren't just great apes", Issues in Ethnology and Anthropology n.s. 3(3): $15-33$.

Dunbar, Robin (2012) "On the evolutionary function of song and dance", in N. Bannan (ed), Music, language and human evolution (Oxford: Oxford University Press), pp. 201-214.

Dunbar, Robin (2014a) Human evolution (London: Pelican).

Dunbar, Robin (2014b) "How conversations around campfires came to be", Proceedings of the National Academy of Sciences USA 111: 14013-14014.

Dunbar, Robin, Kostas Kaskatis, Ian MacDonald and Vinnie Barra (2012) "Performance of music elevates pain threshold and positive affect: implications for the evolutionary function of music", Evolutionary Psychology 10(4): 688-702.

Dutton, Denis (2009) The art instinct (Oxford: Oxford University Press).

Dyck, John (2016) "Natural sounds and musical sounds: a dual distinction", Journal of Aesthetics and Art Criticism 74(3): 291-302.

Eerola, Tuomas (2012) "Modeling listeners' emotional response to music", Topics in Cognitive Science 4(4): 607-624.

Eklund, Matti (2005) "Fiction, indifference, and ontology", Philosophy and Phenomenological Research 71(3): 557-579. 
Eklund, Matti (2011) "Fictionalism", in E. Zalta (ed), Stanford encyclopedia of philosophy (Fall 2011 edition), URL $=<$ http://plato.stanford.edu/archives/fall2011/entries/fictionalism/ $>$. Accessed 05 April 2012.

Ellis, Catherine (1985) Aboriginal music: education for living (Queensland: University of Queensland Press).

Epsi-Sanchis, Pedro and Nicholas Bannan (2012) "Found objects in the musical practices of huntergatherers: implications for the evolution of instrumental music", in N. Bannan (ed), Music, language and human evolution (Oxford: Oxford University Press), pp. 173-198.

Ereshefsky, Marc (1992) “Eliminative pluralism”, Philosophy of Science 59(4): 671-690.

Ereshefsky, Marc (1998) “Species pluralism and anti-realism”, Philosophy of Science 65(1): 103-120.

Ereshefsky, Marc (2010) "Species", in E. Zalta (ed), Stanford encyclopedia of philosophy (Fall 2010 edition), URL= <http://plato.stanford.edu/archives/fall2010/entries/species/>. Accessed 7 January 2016.

Ericsson, K. Anders, Ralf Krampe and Clemens Tesch-Römer (1993) "The role of deliberate practice in the acquisition of expert performance", Psychological Review 100(3): 363-406.

Esparza, Tlacael Miguel, Juan Pablo Bello and Eric Humphrey (2015) "From genre classification to rhythmic similarity: computational and musicological insights”, Journal of New Music Research 44(1): 39-57.

Evans, Gareth (1980) "Things without the mind", in Z. van Straaten (ed), Philosophical subjects: essays presented to P. F. Strawson (Oxford: Clarendon Press), pp. 76-116.

Evans, Jonathan St. B. T. (2003) "In two minds: dual-process accounts of reasoning", Trends in Cognitive Sciences 7(10): 454-459.

Falk, Dean (2000) "Hominid brain evolution and the origins of music", in N. Wallin, B. Merker, S. Brown (eds), Origins of music (Cambridge: MIT Press), pp. 197-216.

Falk, Dean (2004) “Prelinguistic evolution in early hominins: whence motherese?", Behavioral and Brain Sciences 27: 491-541.

Feld, Steven (1982) Sound and sentiment: birds, weeping, poetics, and song in Kaluli expression (Philadelphia: University of Pennsylvania Press).

Ferreri, Laura, Emmanuel Bigand, Patrick Bard and Aurélia Bugaiska (2015) “The influence of music on prefrontal cortex during episodic encoding and retrieval of verbal information: a multichannel fNIRS study", Behavioural Neurology 501: 707625.

Field, Hartry (1980) Science without numbers (Oxford: Blackwell).

Field, Hartry (1989) Realism, mathematics, and modality (Oxford: Blackwell).

Finlayson, Clive, José Carrión, Kimberly Brown, Geraldine Finlayson, Antonio Sánchez-Marco, Darren Fa, Joaquín Rodríguez-Vidal, Santiago Fernández, Elena Fierro, Marco Bernal-Gómez and Francisco Giles-Pacheco (2011) "The Homo habitat niche: using the avian fossil record to depict ecological characteristics of Palaeolithic Eurasian hominins", Quaternary Science Reviews 30: 1525-1532. 
Fitch, W. Tecumseh (2000) "The phonetic potential of nonhuman vocal tracts: comparative cineradiographic observations of vocalising animals", Phonetica 57: 205-218.

Fitch, W. Tecumseh (2009) "Biology of music: another one bites the dust", Current Biology 19(10): 403-404.

Fitch, W. Tecumseh (2015) "Four principles of bio-musicology", Philosophical Transactions B: Biological Sciences 370(1664), doi. 10.1098/rstb.2014.0091.

Fitch, W. Tecumseh and Klaus Zuberbühler (2013) "Primate precursors to human language: beyond discontinuity", in E. Altenmüller, S. Schmidt, E. Zimmerman (eds), Evolution of emotional communication (Oxford: Oxford University Press), pp. 26-48.

Fitzgerald, Timothy (2000) The ideology of religious studies (New York: Oxford University Press).

Flintoff, Brian (2004) Taonga puoro, singing treasures: the musical instruments of the Māori (New Zealand: Craig Potton Publishing).

Foley, Robert and Clive Gamble (2009) "The ecology of social transitions in human evolution", Philosophical Transactions of the Royal Society B: Biological Sciences 364: 3267-3279.

Ford, John (1991) "Vocal traditions among resident killer whales (Orcinus orca) in coastal waters of British Columbia", Canadian Journal of Zoology 69(6): 1454-1483.

Fowler, Gregory (2013) “Against the primary sound account of echoes”, Analysis 73(3): 466-473.

Friend, Stacie (2007) “Fictional characters”, Philosophical Compass 2(2): 141-156.

Gamble, Clive, John Gowlett and Robin Dunbar (2011) "The social brain and the shape of the Palaeolithic”, Cambridge Archaeological Journal 21(1): 115-135.

Gamble, Clive, John Gowlett and Robin Dunbar (2014) Thinking big: how the evolution of social life shaped the human mind (London: Thames \& Hudson).

Gardiner, Martin (2008) "Music training, engagement with sequence, and the development of the natural number concept in young learners", Behavioural and Brain Sciences 31(6): 652-653.

Gaut, Berys (2000) “'Art' as a cluster concept”, in N. Carroll (ed), Theories of art today (Madison: University of Wisconsin Press), pp. 25-44.

Gaywood, Harriet Rosemary Ann (1996) Guqin and guzheng: the historical and contemporary development of two Chinese musical instruments. Master's Thesis, Durham University.

Gazzola, Andrea, Elisa Avanzinelli, Lorenza Mauri, Massimo Scandura and Marco Apollonio (2002) "Temporal changes of howling in south European wolf packs", Italian Journal of Zoology 69(2): 157-161.

Geissmann, Thomas (2002) "Duet-splitting and the evolution of gibbon songs", Biological Reviews 77: 57-76.

Gerhardt, Sue (2004) Why love matters: how affection shapes a baby's brain (London: Routledge).

Gerrans, Philip and David Sander (2014) "Feeling the future: prospects for a theory of implicit prospection”, Biology and Philosophy 29(5): 699-710. 
Gese, Eric and Robert Ruff (1998) "Howling by coyotes (Canis latrans): variation among social classes, seasons, and pack sizes" Canadian Journal of Zoology 76(6): 1037-1043.

Gibbons, Ann (2015) “Deep roots for the genus Homo”, Science 347(6226): 1056-1057.

Godfrey-Smith, Peter (2014a) Philosophy of biology (New Jersey: Princeton University Press).

Godfrey-Smith, Peter (2014b) "Signs and symbolic behaviour", Biological Theory 9(1): 78-88.

Godt, Irving (2005) “Music: a practical definition”, Musical Times 146: 83-88.

Goehr, Lydia (2007) The imaginary museum of musical works, revised edition (Oxford: Oxford University Press).

Goodall, Howard (2013) The story of music (London: Vintage).

Goodman, Nelson (1976) Languages of art, revised edition (Cambridge: Hackett).

Goren-Inbar, Naama, Nira Alperson, Mordechai Kislev, Orit Simchoni, Yoel Melamed, Adi Ben-Nun and Ella Werker (2004) "Evidence of hominin control of fire at Gesher Benot Ya'aqov, Israel", Science 304(5671): 725-727.

Goren-Inbar, Naama, Gonen Sharon, Nira Alperson-Afil and Gadi Herzlinger (2015) "A new type of anvil in the Acheulian of Gesher Benot Ya'aqov, Israel", Philosophical Transactions of the Royal Society: Biological Sciences, doi. 10.1098/rstb.2014.0353.

Gould, Richard (1969) Yiwara: foragers of the Australian desert (London: Collins).

Gould, Stephen Jay (1977) Ontogeny and phylogeny (Cambridge: Harvard University Press).

Gould, Stephen Jay (1997) "The exaptive excellence of spandrels as a term and prototype", Proceedings of the National Academy of Sciences USA 94: 10750-10755.

Gould, Stephen Jay and Richard Lewontin (1978) "The spandrels of San Marco and the Panglossian paradigm: a critique of the adaptationist programme", Proceedings of the Royal Society of London B: Biological Sciences 205: 581-598.

Gould, Stephen Jay and Elizabeth Vrba (1982) "Exaptation—a missing term in the science of form", Paleobiology 8(1): 4-15.

Gowlett, John, Clive Gamble and Robin Dunbar (2012) "Human evolution and the archaeology of the social brain", Current Anthropology 53(6): 693-722.

Gracyk, Theodore (1996) Rhythm and noise: an aesthetics of rock (North Carolina: Duke University Press).

Grahn, Jessica (2012) "Neural mechanisms of rhythm perception: current findings and future perspectives", Topics in Cognitive Science 4(4): 585-606.

Gray, Russell, David Bryant and Simon Greenhill (2010) "On the shape and fabric of human history", Philosophical Transactions of the Royal Society B: Biological Sciences 365: 3923-3933.

Gray, Russell, Quentin Atkinson and Simon Greenhill (2011) "Language, evolution and human history", Philosophical Transactions of the Royal Society B: Biological Sciences 366: 1090-1100. 
Grieser, DiAnne and Patricia Kuhl (1988) "Maternal speech to infants in a tonal language: support for universal prosodic features in motherese", Developmental Psychology 24(1): 14-20.

Griffiths, Paul (2002) “What is innateness?”, Monist 85(1): 70-85.

Griffiths, Paul (2006) "Function, homology and character individuation", Philosophy of Science 73: $1-25$.

Griffiths, Paul and Karola Stotz (2013) Genetics and philosophy: an introduction (New York: Cambridge University Press).

Gupta, Amita (2014) Diverse early childhood education policies and practices: voices and images from five countries in Asia (New York: Routledge).

Gustison, Morgan, Aliza le Roux and Thore Bergman (2012) "Derived vocalizations of geladas (Theropithecus gelada) and the evolution of vocal complexity in primates", Philosophical Transactions of the Royal Society B: Biological Sciences 367: 1847-1859.

Hagel, Stefan (2010) Ancient Greek music: a new technical history (Cambridge: Cambridge University Press).

Hagen, Edward and G. Bryant (2003) “Music and dance as a coalitional signalling system”, Human Nature 14: 21-51.

Hagen, Edward and Peter Hammerstein (2009) “Did Neanderthals and other early humans sing? Seeking the biological roots of music in the territorial advertisements of primates, lions, hyenas, and wolves", Musicae Scientiae 13(suppl-2): 291-320.

Hambrick, David and Elliot Tucker-Drob (2015) "The genetics of music accomplishment: evidence for gene-environment correlation and interaction”, Psychonomic Bulletin and Review 22(1): 112-120.

Hamilton, Andy (2007) Aesthetics and music (London: Continuum).

Hamilton, Andy (2009) "The sound of music", in M. Nudds, C. O'Callaghan (eds), Sounds and perceptions: new philosophical essays (New York: Oxford University Press), pp. 146-182.

Hannon, Erin and Laurel Trainor (2007) "Music acquisition: effects of enculturation and formal training on development", Trends in Cognitive Science 11(11): 466-472.

Harmand, Sonia, Jason Lewis, Craig Feibel, Christopher Lepre, Sandrine Prat, Arnaud Lenoble, Xavier Boës, Rhonda Quinn, Michel Brenet, Adrian Arroyo, Nicholas Taylor, Sophie Clément, Guillaume Daver, Jean-Philip Brugal, Louise Leaky, Richard Mortlock, James Wright, Sammy Lokorodi, Christopher Kirwa, Dennis Kent and Hélène Roche (2015) "3.3-million-year-old stone tools from Lomekwi 3, West Turkana, Kenya”, Nature 521: 310-315.

Harrington, Fred (1989) "Chorus howling by wolves: acoustic structure, pack size and the Beau Geste effect", Bioacoustics 2(2): 117-136.

Hattori, Yuko, Masaki Tomonaga and Tetsuro Matsuzawa (2015) "Spontaneous synchronized tapping to an auditory rhythm in a chimpanzee", Scientific Reports 3: 1566.

Hawkes, Kristin (2003) "Grandmothers and the evolution of human longevity", American Journal of Human Biology 15: 380-400.

Healey, Tim (1994) Life in the land of the pharaohs (London: Toucan Books/Reader's Digest). 
Hendy, David (2013) Noise: a human history of sound and listening (London: Profile).

Henshilwood, Christopher, Francesco d'Errico and Ian Watts (2009) "Engraved ochres from the Middle Stone Age levels at Blombos Cave, South Africa", Journal of Human Evolution 57(1): 27-47.

Hepper, Peter (1991) “An examination of fetal learning before and after birth", Irish Journal of Psychology 12(2): 95-107.

Hewlett, Barry and L. L. Cavalli-Sforza (1986) “Cultural transmission among Aka Pygmies”, American Anthropologist 88(4): 922-934.

Higham, Thomas, Laura Basell, Roger Jacobi, Rachel Wood, Christopher Bronk Ramsey and Nicholas Conard (2012) "Testing models for the beginnings of the Aurignacian and the advent of figurative art and music: the radiocarbon chronology of Geißenklösterle", Journal of Human Evolution 62: 664-676.

Hirsch Jr, E. Donald (1978) “What isn't literature?”, in P. Hernadi (ed), What is literature? (Bloomington: Indiana University Press), pp. 24-34.

Hiscock, Peter (2014) "Learning in Lithic Landscapes: A Reconsideration of the Hominid “Toolmaking” Niche", Biological Theory 9(1): 27-41.

Hobaiter, Catherine and Richard Byrne (2014) "The meanings of chimpanzee gestures", Current Biology 24: 1596-1600.

Hodgson, Derek and Jan Verpooten (2014) "The evolutionary significance of the arts: exploring the by-product hypothesis in the context of ritual, precursors, and cultural evolution", Biological Theory 10(1): $73-85$.

Hoeschele, Marisa, Hugo Merchant, Yukiko Kikuchi, Yuko Hattori and Carel ten Cate (2015) "Searching for the origins of musicality across species", Philosophical Transactions B: Biological Sciences 370(1664), doi. 10.1098/rstb.2014.0094.

Holzapfel, André (2015) "Relation between surface rhythm and rhythmic modes in Turkish makam music", Journal of New Music Research 44(1): 25-38.

Honing, Henkjan, Hugo Merchant, Gábor Háden, Luis Prado and Ramón Bartolo (2012) "Rhesus monkeys (macaca mulatta) detect rhythmic groups in music, but not the beat", PLoS One 7(12): e51369.

Honing, Henkjan and Annemie Ploeger (2012) "Cognition and the evolution of music: pitfalls and prospects", Topics in Cognitive Science 4: 513-524.

Howard, James Henri (1984) The Canadian Sioux (Nebraska: University of Nebraska Press).

Howe, Michael, Jane Davidson and John Sloboda (1998) "Innate talents: reality or myth?" Behavioural and Brain Sciences 21(3): 399-407.

Howell, Robert (2015) “Objects of fiction and objects of thought”, in S. Brock, A. Everett (eds), Fictional objects (Oxford: Oxford University Press), pp. 41-70.

Howell, S., E. Roeder, C. Nelson, J. Fritz and M. Schwandt (2002) "The effect of music on the behaviour of captive chimpanzees (Pan troglodytes)", American Journal of Primatology 57(suppl-1): 83-84. 
Hrdy, Sarah (2009) Mothers and others: the evolutionary origins of mutual understanding (Cambridge: Harvard University Press).

Hume, David (1748) An enquiry concerning human understanding (London: Millar).

Huron, David (2011) "Why is sad music pleasurable? A possible role for prolactin", Musicae Scientiae 15(2): 146-158.

Hussain, Nadeem (2010) "Error theory and fictionalism", in J. Skorupski (ed), Routledge companion to ethics (Oxon: Routledge), pp. 335-345.

Ingarden, Roman (1989) Ontology of the work of art [R. Meyer with J.T. Goldthwait, trans.] (Athens: Ohio University Press).

Jackson, Frank (1998) From metaphysics to ethics: a defence of conceptual analysis (Oxford: Clarendon Press).

Jackson, Frank and Philip Pettit (1995) "Moral functionalism and moral motivation”, Philosophical Quarterly 45(178): 20-40.

James, William (2004) [1907] Pragmatism (Project Gutenberg).

Jeffares, Ben (2010) "The co-evolution of tools and minds: cognition and material culture in the hominin lineage", Phenomenology and the Cognitive Sciences 9(4): 503-520.

Johansson, Sverker (2015) “Language abilities in Neanderthals", Annual Review of Linguistics 1: 311-332.

Jordania, Joseph (2011) Why do people sing? Music in human evolution (Tbilisi: Logos).

Jourdain, Robert (1997) Music, the brain, and ecstasy: how music captures our imagination (New York: Harper Perennial).

Joyce, Richard (2005) "Moral fictionalism", in M. E. Kalderon (ed), Fictionalism in metaphysics (New York: Oxford University Press), pp. 287-313.

Kahneman, Daniel (2011) Thinking, fast and slow (London: Allen Lane).

Kandel, Eric, James Harris Schwartz and Thomas Jessell (1995) Essentials of neural science and behavior (Connecticut: Appleton \& Lange).

Kania, Andrew (2005) "Review of Art as performance", Mind 114: 137-141.

Kania, Andrew (2006) "Making tracks: the ontology of rock music", Journal of Aesthetics and Art Criticism 64(4): 401-414.

Kania, Andrew (2008) "The methodology of musical ontology: descriptivism and its implications", British Journal of Aesthetics 48(4): 426-444.

Kania, Andrew (2010) "Silent music", Journal of Aesthetics and Art Criticism 68: 343-353.

Kania, Andrew (2011) "Definition", in T. Gracyk, A. Kania (eds), Routledge companion to philosophy and music (London: Routledge), pp. 3-13.

Kania, Andrew (2012a) "Platonism vs nominalism in contemporary musical ontology", in C. Mag Uidhir (ed), Art and abstract objects (Oxford: Oxford University Press), pp. 197-219. 
Kania, Andrew (2012b) "The philosophy of music", in E. Zalta (ed), Stanford encyclopedia of philosophy (Fall 2012 edition), URL= <http://plato.stanford.edu/archives/fall2012/entries/music/>. Accessed 12 March 2016.

Kehoe, Alice (1999) "Blackfoot and other hunters on the North American Plains", in R. Lee, R. Daly (eds), The Cambridge encyclopedia of hunters and gatherers (Cambridge: Cambridge University Press), pp. 36-40.

Kendal, Jeremy, Jamshid Tehrani and John Odling-Smee (2011) "Human niche construction in interdisciplinary focus" Philosophical Transactions of the Royal Society B: Biological Sciences 366: 785-792.

Keverne, Eric, Nicholas Martensz and Bernadette Tuite (1989) "Beta-endorphin concentrations in cerebrospinal fluid of monkeys are influenced by grooming relationships", Psychoneuroendocrinology 14: $155-161$.

Killin, Anton (2013) "The arts and human nature: evolutionary aesthetics and the evolutionary status of art behaviours", Biology and Philosophy 28(4): 703-718.

Killin, Anton (2014) "Musicality in human evolution, archaeology and ethnography", Biology and Philosophy 29(4): 597-609.

Killin, Anton (2016a) “Rethinking music's status as adaptation versus technology: a niche construction perspective", Ethnomusicology Forum 25(2): 210-233.

Killin, Anton (2016b) "Musicality and the evolution of mind, mimesis, and entrainment", Biology and Philosophy 31(3): 421-434.

Killin, Anton (2017) "Music and human evolution: philosophical aspects", in R. Joyce (ed), Routledge companion to evolution and philosophy (Oxon: Routledge).

Kimura, Doreen (1993) Neuromotor mechanisms in human communication (Oxford: Oxford University Press).

Kinsley, David (1988) Hindu goddesses: vision of the divine feminine in the Hindu religious traditions (California: University of California Press).

Kitchen, Dawn, Rogério Grassetto Teixeira de Cunha, Ingrid Holzmann and Dilmar Alberto Gonçalves de Oliveira (2015) "Function of loud calls in howler monkeys", in M. M. Kowalewski, P. A. Garber, L. Cortés-Ortiz , B. Urbani, D. Youlatos (eds), Howler monkeys: adaptive radiation, systematics, and morphology (New York: Springer), pp. 369-399.

Kitcher, Philip (1984) “Species”, Philosophy of Science 51: 308-333.

Kivy, Peter (1983) “Platonism in music: a kind of defense", Grazer Philosophische Studien 19: 109-129.

Kivy, Peter (1987) "Platonism in music: another kind of defense", American Philosophical Quarterly 24(3): 245-252.

Kivy, Peter (1991) “Is music an art?”, Journal of Philosophy 88(10): 544-554.

Kivy, Peter (2002) Introduction to a philosophy of music (New York: Oxford University Press).

Klein, Devorah and Gregory Murphy (2001) "The representation of polysemous words", Journal of Memory and Language 45: 259-282. 
Klein, Devorah and Gregory Murphy (2002) "Paper has been my ruin: conceptual relations of polysemous senses", Journal of Memory and Language 47: 548-570.

Klein, Richard (2000), "Archaeology and the evolution of human behavior", Evolutionary Anthropology 9(1): 17-36.

Klein, Richard (2009) The human career: human biological and cultural origins (Chicago: University of Chicago Press).

Klein, Richard (2013) “Modern human origins”, General Anthropology 20: 1-4.

Klein, Richard and Blake Edgar (2002) The dawn of human culture (New York: Wiley).

Klepousniotou, Ekaterini and Shari Baum (2007) "Disambiguating the ambiguity advantage for word recognition: an advantage for polysemous but not homonymous words", Journal of Neurolinguistics 20: $1-24$.

Kohn, Marek and Steven Mithen (1999) "Handaxes: products of sexual selection”, Antiquity 73: 518526.

Kramer, Karen (2011) “The evolution of human parental care and recruitment of juvenile help", Trends in Ecology and Evolution 26(10): 533-540.

Kripke, Saul (1977) "Speaker's reference and semantic reference”, Midwest Studies in Philosophy 2(1): $255-276$.

Kristeller, Paul (1951) "The modern system of the arts: a study in the history of aesthetics (I)", Journal of the History of Ideas 12(4): 496-527.

Kristeller, Paul (1952) "The modern system of the arts: a study in the history of aesthetics (II)", Journal of the History of Ideas 13(1): 17-46.

Kroon, Frederick (2011) “Fictionalism in metaphysics”, Philosophy Compass 6(11): 786-803.

Kruuk, Hans (1972) The spotted hyena: a study of predation and social behaviour (Chicago: Chicago University Press).

Kuhn, Steven and Mary Stiner (1998) "The earliest Aurignacian of Riparo Mochi (Liguria, Italy)", Current Anthropology 39(suppl-3): 175-189.

Kulvicki, John (2008) “The nature of noise”, Philosophers' Imprint 8(11): 1-16.

Laland, Kevin and Michael O’Brien (2011) “Cultural niche construction: an introduction”, Biological Theory 6: 191-202.

Laland, Kevin, John Odling-Smee, William Hoppitt and Tobias Uller (2013) "More on how and why: cause and effect in biology revisited", Biology and Philosophy 28(5): 719-745.

Larsson, Matz (2014) "Self-generated sounds of locomotion and ventilation and the evolution of human rhythmic abilities", Animal Cognition 17: 1-14.

Larsson, Matz (2015) “Tool-use-associated sound in the evolution of language”, Animal Cognition 18: 993-1005.

Lawergren, Bo (1988) “The origin of musical instruments and sounds", Anthropos 83: 31-45. 
Lawson, Andrew (2012) Painted caves: Palaeolithic rock art in western Europe (Oxford: Oxford University Press).

Lawson, Francesca R. Sborgi (2014) "Is music an adaptation or a technology? Ethnomusicological perspectives from the analysis of Chinese shuochang", Ethnomusicology Forum 23(1): 3-26.

Leaky, Mary (1971) Olduvai Gorge, excavations in Beds I and II, 1960-1963 (Cambridge: Cambridge University Press).

Leaky, Mary and John Harris (1987) Laetoli: a Pliocene site in northern Tanzania (New York: Oxford University Press).

Lebens, Samuel (2015) “Would this paper exist if I hadn't written it?”, Philosophical Studies 172(11): 3059-3080.

Leng, Mary (2005) “Revolutionary fictionalism: a call to arms", Philosophia Mathematica 13(3): $277-293$.

Lennox, John (2001) "History and philosophy of science: a phylogenetic approach", Historia, Ciencias, Saude-Manguinhos 8: 655-669.

Lepre, Christopher, Hélène Roche, Dennis Kent, Sonia Harmand, Rhonda Quinn, Jean-Philippe Brugal, Pierre-Jean Texier, Arnaud Lenoble and Craig Feibel (2011) "An earlier origin for the Acheulian", Nature 477: 82-85.

Letts, Philip (2015) “Against Kania's fictionalism about musical works", British Journal of Aesthetics 55(2): 209-224.

Levinson, Jerrold (1980) "What a musical work is", Journal of Philosophy 77(1): 5-28.

Levinson, Jerrold (1990) Music, art, and metaphysics (Ithaca: Cornell University Press).

Levitin, Daniel (2006) This is your brain on music: understanding a human obsession (London: Atlantic Books).

Lewens, Tim (2012) “Pheneticism reconsidered”, Biology and Philosophy 27(2): 159-177.

Lewis, David (1986) On the plurality of worlds (Oxford: Blackwell).

Lewis-Williams, David (2004) The mind in the cave (London: Thames \& Hudson).

Liebenberg, Louis (1990) The art of tracking: the origin of science (Claremont: D. Philip).

Livingston, Paisley (2011) "History of the ontology of art", in E. Zalta (ed), Stanford encyclopedia of philosophy (Fall 2011 edition), URL= <http://plato.stanford.edu/archives/fall2011/entries/art-ontologyhistory/>. Accessed 30 May 2012.

Lock, Andy and Matt Gers (2012) "The cultural evolution of written language and its effects: a Darwinian process from prehistory to the modern day", in E. L. Grigorenko, E. Mambrino, D. D. Preiss (eds), Writing: a mosaic of new perspectives (New York: Psychology Press), pp. 11-35.

London, Justin (2012) "Schemas, not syntax: a reply to Patel”, in P. Rebuschat, M. Rohrmeier, J. A. Hawkins, I. Cross (eds), Language and music as cognitive systems (Oxford: Oxford University Press), pp. 242-247.

Lopes, Dominic Mclver (2010) A philosophy of computer art (Oxon: Routledge). 
Lopes, Dominic Mclvor (2014) Beyond art (Oxford: Oxford University Press).

Loui, Psyche (2012) "Learning and liking of melody and harmony: further studies in artificial grammar learning", Topics in Cognitive Science 4(4): 554-567.

Love, Alan (2008) "Explaining evolutionary innovations and novelties: criteria of explanatory adequacy and epistemological prerequisites", Philosophy of Science 75(5): 874-886.

MacCormack, Carol (1980) "Proto-adult to adult: a Sherbro transformation", in C. P. MacCormack, M. Strathern (eds), Nature, culture and gender (Cambridge: Cambridge University Press), pp. 95-118.

Mackie, John (1977) Ethics: inventing right and wrong (London: Pelican).

Maclachlan, D. C. L. (1989) Philosophy of perception (Englewood Cliffs: Prentice Hall).

MacLarnon, Ann and Gwen Hewitt (1999) "The evolution of human speech: the role of enhanced breathing control”, American Journal of Physical Anthropology 109: 341-363.

Maclaurin, James and Kim Sterelny (2008) What is biodiversity? (Chicago: University of Chicago Press).

Maconie, Robin (1990) The concept of music (Oxford: Clarendon Press).

Mag Uidhir, Christy (2007) “Recordings as performances”, British Journal of Aesthetics 47(3): 298-314.

Mag Uidhir, Christy and P. D. Magnus (2011) “Art concept pluralism”, Metaphilosophy 42: 83-97.

Malafouris, Lambros (2010) "Metaplasticity and the human becoming: principles of neuroarchaeology", Journal of Anthropological Sciences 88: 49-72.

Malloch, Stephen and Colwyn Trevarthen (eds) (2010) Communicative musicality: exploring the basis of human companionship (Oxford: Oxford University Press).

Malm, William (1967) Music cultures of the Pacific, the Near East, and Asia (New Jersey: Prentice-Hall).

Mameli, Matteo (2008) "On innateness: the clutter hypothesis and the cluster hypothesis", Journal of Philosophy 105(12): 719-736.

Mameli, Matteo and Patrick Bateson (2006) "Innateness and the sciences", Biology and Philosophy 21(2): $155-188$.

Mameli, Matteo and Patrick Bateson (2011) "An evaluation of the concept of innateness", Philosophical Transactions of the Royal Society B: Biological Sciences 366: 436-443.

Manniche, Lise (1991) Music and musicians in Ancient Egypt (London: British Museum Press).

Marcus, Gary (2012) “Musicality: instinct or acquired skill?”, Topics in Cognitive Science 4(4): 498-512.

Margulis, Elizabeth (2014) On repetition (Oxford: Oxford University Press).

Marlowe, Frank (2010) The Hadza: hunter-gatherers of Tanzania (Berkeley: University of California Press).

Martin, Robert (1993) "Musical works in the worlds of performers and listeners", in M. Krausz (ed), The interpretation of music: philosophical essays (New York: Oxford University Press), pp. 119-127. 
Marwick, Ben (2003) "Pleistocene exchange networks as evidence for the evolution of language", Cambridge Archaeological Journal 13(1): 67-81.

Marzke, Mary (2013) "Tool making, hand morphology and fossil hominins", Philosophical Transactions of the Royal Society B: Biological Sciences, doi. 10.1098/rstb.2012.0414.

Matsusaka, Takahisa (2012) "Playful drumming by immature wild chimpanzees at Mahale: do they enjoy making sounds?”, Pan Africa News 19(2): 23-25.

Matthen, Mohan (2010) "On the diversity of auditory objects", Review of Philosophy and Psychology 1(1): 63-89.

Mayr, Ernst (1961) “Cause and effect in biology”, Science 134(3489): 1501-1506.

McAllester, David (1996) "North America/Native America”, in J. Titon (ed), Worlds of Music: an introduction to the music of the world's people (New York: Schirmer), pp. 33-82.

McBrearty, Sally and Andrew Brooks (2000) “The revolution that wasn't: a new interpretation of the origin of modern human behaviour", Journal of Human Evolution 39: 453-563.

McBrearty, Sally and Chris Stringer (2007) “Palaeoanthropology: the coast in colour”, Nature 449: 793-794.

McDermott, Josh and Marc Hauser (2005) "The origins of music: innateness, uniqueness, and evolution", Music Perception 23(1): 29-59.

McDermott, Josh, Alan Schultz, Eduardo Undurraga and Ricardo Godoy (2016) "Indifference to dissonance in native Amazonians reveals cultural variation in music perception", Nature 535: 547-550.

McGovern, Patrick (2009) Uncorking the past: the quest for wine, beer and other alcoholic beverages (California: University of California Press).

McKeown-Green, Jonathan (2014) “What is music? Is there a definitive answer?”, Journal of Aesthetics and Art Criticism 72: 393-403.

McLean, Mervyn (1996) Māori music (New Zealand: Auckland University Press).

McNabb, John, Francesca Binyon and Lee Hazelwood (2004) "The large cutting tools from South African Acheulean and the question of social traditions", Current Anthropology 45: 653-677.

McPherron, Shannon, Zeresenay Alemseged, Curtis Marean, Jonathan Wynn, Denné Reed, Denis Geraads, René Bobe and Hamdallah Béarat (2010) "Evidence for stone-tool-assisted consumption of animal tissues before 3.39 million years ago at Dikkia, Ethiopia", Nature 466: 857-860.

Meehan, Maureen (1997) "Police beatings and torture in Israel”, Washington Report on Middle East Affairs 15(6): 7.

Meinong, Alexius (1904) "Uber Gegenstandstheorie", in A. Meinong (ed), Untersuchungen zur Gegenstandstheorie und Psychologie (Leipzig: J.A. Barth), pp. 1-51.

Menary, Richard (2014) “The aesthetic niche”, British Journal of Aesthetics 54(4): 471-475.

Merchant, Hugo, Jessica Grahn, Laurel Trainor, Martin Rohrmeier and W. Tecumseh Fitch (2015) "Finding the beat: a neural perspective across humans and non-human primates", Philosophical Transactions B: Biological Sciences 370(1664), doi. 10.1098/rstb.2014.0093. 
Merchant, Hugo and Henkjan Honing (2014) "Are non-human primates capable of rhythmic entrainment? Evidence for the gradual audiomotor evolution hypothesis", Frontiers in Neuroscience 7: 274 .

Merker, Björn (2012) “The vocal learning constellation: imitation, ritual culture, encephalization”, in N. Bannan (ed), Music, language and human evolution (Oxford: Oxford University Press), pp. 215-260.

Merker, Björn, lain Morley and Willem Zuidema (2015) "Five fundamental constraints of theories of the origins of music", Philosophical Transactions of the Royal Society: Biological Sciences, 370: doi. 10.1098/rstb.2014.0095.

Merriam, Alan (1963) “Purposes of ethnomusicology, an anthropological view", Ethnomusicology 7(3): 206-213.

Merriam, Alan (1964) The anthropology of music (Chicago: Northwestern University Press).

Metcalf, Peter and Richard Huntington (1991) Celebrations of death: the anthropology of mortuary ritual, 2nd edition (Cambridge: Cambridge University Press).

Miller, Geoffrey (2000) "Evolution of human music through sexual selection", in N. Wallin, B. Merker, S. Brown (eds), Origins of music (Cambridge: MIT Press), pp. 329-360.

Miller, Geoffrey (2001) The mating mind: how sexual choice shaped the evolution of human nature (London: Vintage).

Mills, Steve (2014) Auditory archaeology: understanding sound and hearing in the past (California: Left Coast Press).

Mithen, Steven (1996) The prehistory of the mind: a search for the origins of art, religion and science (Great Britain: Thames \& Hudson).

Mithen, Steven (2002) "Human evolution and the cognitive basis of science", in P. Carruthers, S. Stitch, M. Siegal (eds), Cognitive basis of science (New York: Cambridge University Press), pp. 23-40.

Mithen, Steven (2005) The Singing Neanderthals: the origins of music, language, mind and body (Great Britain: Weidenfeld \& Nicolson).

Monseré, Annelies (2012) "Non-Western art and the concept of art: can cluster theories of art account for the universality of art?", Estetika: The Central European Journal of Aesthetics 49(2): $148-165$.

Montelle, Yann-Pierre (2004) "Paleoperformance: investigating the human use of caves in the Upper Paleolithic", in G. Bergaus (ed), New perspectives on prehistoric art (Westport: Praeger), pp. 131-152.

Moore, Ignacio, George Bentley, Cheryl Wotus and John Wingfield (2006) “Photoperiodindependent changes in immunoreactive brain gonadotropin-releasing hormone $(\mathrm{GnRH})$ in a freeliving, tropical bird)", Brain, Behaviour and Evolution 68(1): 37-44.

Morley, lain (2013) The prehistory of music: human evolution, archaeology and the origins of musicality (Oxford: Oxford University Press).

Mosing, Miriam, Karin Verweij, Guy Madison, Nancy Pedersen, Brendan Zietsch, and Fredrik Ullén (2015) "Did sexual selection shape human music? Testing predictions from the sexual selection hypothesis of music evolution using a large genetically informative sample of over 10,000 twins", Evolution and Human Behavior 36(5): 359-366. 
Nercessian, Andy (2007) Defining music: an ethnomusicological and philosophical approach (New York: Edwin Mellen Press).

Nettl, Bruno (1990) Folk and traditional music of the Western continents, $3^{\text {rd }}$ edition (New Jersey: Prentice-Hall).

Nettl, Bruno (2005) The study of ethnomusicology: thirty-one issues and concepts (Chicago: University of Illinois Press).

Neuhaus, Christiane (2013) "Processing musical form: behavioural and neurocognitive approaches", Musicae Scientiae 17(1): 109-127.

Newman, Aaron, Daphne Bavelier, David Corina, Peter Jezzard and Helen Neville (2002) "A critical period for right hemisphere recruitment in American Sign Language processing", Nature Neuroscience 5: 76-80.

Nolan, Daniel (2013) "Why historians (and everyone else) should care about counterfactuals", Philosophical Studies 163: 317-335.

Nolan, Daniel (2015) “The a posteriori armchair”, Australasian Journal of Philosophy 93(2): 211-231.

Nowell, April and Melanie Lee Chang (2009) "The case against sexual selection as an explanation of handaxe morphology", Paleoanthropology 2009: 77-88.

Nudds, Matthew (2009) "Sounds and space", in M. Nudds, C. O'Callaghan (eds), Sounds and perceptions: new philosophical essays (New York: Oxford University Press), pp. 69-96.

Nudds, Matthew and Casey O'Callaghan (eds) (2009) Sounds and perceptions: new philosophical essays (New York: Oxford University Press).

Nunns, Richard (2014) Te ara puoro: a journey into the world of Māori music (New Zealand: Craig Potton Publishing).

O’Callaghan, Casey (2007a) “Echoes”, Monist 90: 403-414.

O'Callaghan, Casey (2007b) Sounds: a philosophical theory (Oxford: Oxford University Press).

O'Callaghan, Casey (2009a) "Sounds and events", in M. Nudds, C. O'Callaghan (eds), Sounds and perceptions: new philosophical essays (New York: Oxford University Press), pp. 26-49.

O'Callaghan, Casey (2009b) “Constructing a theory of sounds", in D.W. Zimmerman (ed), Oxford Studies in Metaphysics volume 5 (Oxford: Oxford University Press), pp. 247-270.

O'Callaghan, Casey (2010) "Perceiving the locations of sounds", Review of Philosophy and Psychology 1(1): 123-140.

Odling-Smee, John, Kevin Laland and Marcus Feldman (2003) Niche construction: the neglected process in evolution (New Jersey: Princeton University Press).

Odling-Smee, John and Kevin Laland (2011) "Ecological inheritance and cultural inheritance: what are they and how do they differ?", Biological Theory 6: 220-230.

Oppenheim, Paul and Hilary Putnam (1958) "The unity of science as a working hypothesis", in G. Maxwell, H. Feigl, M. Scriven (eds), Concepts, theories, and the mind-body problem (Minneapolis: Minnesota University Press), pp. 3-36. 
Orians, Gordon (2014) Snakes, sunrises, and Shakespeare: how evolution shapes our loves and fears (Chicago: University of Chicago Press).

O'Shaughnessy, Brian (2009) “The location of a perceived sound”, in M. Nudds, C. O'Callaghan (eds), Sounds and perceptions: new philosophical essays (New York: Oxford University Press), pp. 111-125.

Ottenberg, Simon (1989) Boyhood rituals in an African society: an interpretation (Seattle: University of Washington Press).

Panksepp, Jaak (2009) "The emotional antecedents to the evolution of music and language", Musicae Scientiae 13(suppl-2): 225-259.

Papineau, David (2009) “Naturalism", in E. Zalta (ed), Stanford encyclopedia of philosophy (Spring 2009 edition), URL=<http://plato.stanford.edu/archives/spr2009/entries/naturalism/ . Accessed 26 June 2012.

Parncutt, Richard (2009) "Prenatal and infant conditioning, the mother schema, and the origins of music and religion”, Musicae Scientiae 13(suppl-2): 119-150.

Pasnau, Robert (1999) “What is sound?”, The Philosophical Quarterly 49(196): 309-324.

Patel, Aniruddh (2006) "Musical rhythm, linguistic rhythm, and human evolution", Music Perception 24(1): 99-104.

Patel, Aniruddh (2008) Music, language, and the brain (Oxford: Oxford University Press).

Patel, Aniruddh (2010) "Music, biological evolution, and the brain", in M. Bailar, C. Field, C. J. Henry (eds), Emerging disciplines (Houston: Rice University Press), pp. 91-144.

Patel, Aniruddh (2012a) "Language, music, and the brain: a resource-sharing framework", in P. Rebuschat, M. Rohrmeier, J. A. Hawkins, I. Cross (eds), Language and music as cognitive systems (Oxford: Oxford University Press), pp. 204-223.

Patel, Aniruddh (2012b) "Advancing the comparative study of linguistic and musical syntactic processing", in P. Rebuschat, M. Rohrmeier, J. A. Hawkins, I. Cross (eds), Language and music as cognitive systems (Oxford: Oxford University Press), pp. 248-253.

Patel, Aniruddh, John Iversen, Micah Bregman and Irena Schulz (2009) "Experimental evidence for synchronization to a musical beat in a nonhuman animal", Current Biology 19(10): 827-830.

Paul, Robert (2015) Mixed messages: cultural and genetic inheritance in the constitution of human society (Chicago: University of Chicago Press).

Peretz, Isabelle (2011) “Towards a neurobiology of musical emotions”, in P. N. Juslin, J. A. Sloboda (eds), Oxford handbook of music and emotion (Oxford: Oxford University Press), pp. 99-126.

Peretz, Isabelle and Max Coltheart (2003) "Modularity of music processing", Nature Neuroscience 6: 688-691.

Perkins, Moreland (1983) Sensing the world (Indianapolis: Hackett).

Petraglia, Michael, Ceri Shipton and K. Paddayya (2005) "Life and mind in the Acheulean", in

C. Gamble, M. Porr (eds), The individual hominid in context: archaeological investigations of Lower and Middle Palaeolithic landscapes, locales and artefacts (Oxon: Routledge), pp. 197-219. 
Petraglia, Michael and Ceri Shipton (2008) "Large cutting tool variation west and east of the Movius Line", Journal of Human Evolution 55(6): 962-966.

Pfeiffer, John (1982) The creative explosion: an inquiry into the origins of art and religion (New York: Harper \& Row).

Pika, Simone and John Mitani (2006) "Referential gestural communication in wild chimpanzees (Pan troglodytes)”, Current Biology 16(6): R191-192.

Pike, A. W. G., D. L. Hoffmann, M. García-Diez, P. B. Pettitt, J. Alcolea, R. De Balbín, C. González-Sainz, C. de las Heras, J. A. Lasheras, R. Montes and J. Zilhão (2012) "U-series dating of Palaeolithic art in 11 caves in Spain", Science 336: 1409-1413.

Pinker, Steven (1997) How the mind works (London: Allen Lane).

Predelli, Stefano (2001) "Musical ontology and the argument from creation", British Journal of Aesthetics 41(3): 279-292.

Prinz, Jesse (2002) Furnishing the mind: concepts and their perceptual basis (Cambridge: MIT Press).

Quine, Willard (1939) “Designation and existence”, Journal of Philosophy 36(26): 701-709.

Quine, Willard (1960) Word and object (Cambridge: MIT Press).

Ravin, Yael and Claudia Leacock (eds) (2000) Polysemy: theoretical and computational approaches (Oxford: Oxford University Press).

Renfrew, Colin and Paul Bahn (2012) Archaeology: theories, methods and practice, $6^{\text {th }}$ edition (London: Thames \& Hudson).

Rey, Georges (2006) “The intentional inexistence of language-but not cars", in R. Stainton (ed), Contemporary debates in cognitive science (Oxford: Blackwell), pp. 237-255.

Richman, Bruce (1987) “Rhythm and melody in gelada vocal exchanges", Primates 28(2): 199-223.

Richman, Bruce (1993) "On the evolution of speech: singing as the middle term", Current Anthropology 34: 721-722.

Richman, Bruce (2000) "How music fixed "nonsense" into significant formulas: on rhythm, repetition, and meaning", in N. Wallin, B. Merker, S. Brown (eds), Origins of music (Cambridge: MIT Press), pp. 301-314.

Roebroeks, Wil and Paola Villa (2011) "On the earliest evidence for habitual use of fire in Europe", Proceedings of the National Academy of Sciences USA 108: 5209-5214.

Roffman, Itai, Sue Savage-Rumbaugh, Elizabeth Rubert-Pugh, Avraham Ronen and Eviatar Nevo (2012) "Stone tool production and utilization by bonobo-chimpanzees (Pan paniscus)", Proceedings of the National Academy of Sciences USA 109: 14500-14503.

Rohrbaugh, Guy (2003) “Artworks as historical individuals”, European Journal of Philosophy 11(2): 177-205.

Rohrbaugh, Guy (2005) "Ontology of art”, in B. Gaut, D. M. Lopes (eds), Routledge companion to aesthetics, second edition (Oxon: Routledge), pp. 241-253.

Rosen, Gideon (1990) “Modal fictionalism”, Mind 99(395): 327-354. 
Rosen, Gideon (2005) "Problems in the history of fictionalism", in M. E. Kalderon (ed), Fictionalism in metaphysics (New York: Oxford University Press), pp. 14-64.

Rosman, Abraham and Paula Rubel (2004) The tapestry of culture (New York: McGraw-Hill).

Rudner, Richard (1950) "The ontological status of the esthetic object", Philosophy and Phenomenological Research 10(3): 380-388.

Russell, Bertrand (1919) Introduction to mathematical philosophy (London: George Allen \& Unwin Ltd.).

Rzeszutek, Tom, Patrick E. Savage and Steven Brown (2012) "The structure of cross-cultural musical diversity”, Proceedings of the Royal Society B. Biological Sciences 279(1733): 1606-1612.

Sachs, Curt (1943) The rise of music in the ancient world: east and west (New York: Dover).

Sachs, Curt (1962) The wellsprings of music (Netherlands: Martinus Nijhoff).

Sadra, I. Wayan and Jody Diamond (1991) "Komposisi baru: on contemporary composition in Indonesia”, Leonardo Music Journal 1(1): 19-24.

Sainsbury, R. M. (2010) Fiction and fictionalism (Oxon: Routledge).

Sakata, Hiromi Lorraine (1983) Music in the mind (Ohio: Kent State University Press).

Santana, Carlos (2014) "Save the planet: eliminate biodiversity", Biology and Philosophy 29: 761-780.

Sarkar, Sahotra (2014) "Biodiversity and systematic conservation planning for the Twenty-first Century: a philosophical approach", Conservation Science 2: 1-11.

Sartre, Jean-Paul (1940) The psychology of imagination (New York: Philosophical Library).

Savage, Patrick and Steven Brown (2013) "Toward a new comparative musicology", Analytic Approaches to World Music 2(2): 148-197.

Savage, Patrick, Hiromi Matsumae, Hiroki Oota, Mark Stoneking, Thomas Currie, Atsushi Tajima, Matt Gillian and Steven Brown (2015) "How 'circumpolar' is Ainu music? Musical and genetic perspectives on the history of the Japanese archipelago", Ethnomusicology Forum 24(3): 443-467.

Schaal, Nora, Michael Banissy and Kathrin Lange (2015) "The rhythm span task: comparing memory capacity for musical rhythms in musicians and non-musicians", Journal of New Music Research 44(1): 3-10.

Schachner, Adena (2013) "The origins of human and avian auditory-motor entrainment", Nova Acta Leopoldina 111: 243-253.

Schaffrath, Helmut (1995) The Essen Folksong Collection [D. Huron, ed.] (Stanford: Center for Computer-Assisted Research in the Humanities).

Schellenberg, E. Glenn. (2004) “Music lessons enhance IQ”, Psychological Science 15(8): 511-514.

Schellenberg, E. Glenn (2005) “Music and cognitive abilities", Current Directions in Psychological Science 14(6): 317-320.

Schellenberg, E. Glenn (2006) "Long-term positive associations between music lessons and IQ", Journal of Educational Psychology 98(2): 457-468. 
Schellenberg, E. Glenn (2016) "Music training and nonmusical abilities", in S. Hallam, I. Cross, M. Thaut (eds), Oxford handbook of music psychology, second edition (Oxford: Oxford University Press), pp. 415-429.

Schellenberg, E. Glenn and Laurel Trainor (1996) "Sensory consonance and the perceptual similarity of complex-tone harmonic intervals: tests of adult and infant listeners", Journal of the Acoustical Society of America 100(5): 3321-3328.

Schellenberg, E. Glenn and Sandra Trehub (1996a) "Children's discrimination of melodic intervals", Developmental Psychology 32(6): 1039-1050.

Schellenberg, E. Glenn and Sandra Trehub (1996b) "Natural intervals in music: a perspective from infant listeners", Psychological Science 7(5): 272-277.

Schellenberg, E. Glenn and Sandra Trehub (1999) "Culture-general and culture-specific factors in the discrimination of melodies", Journal of Experimental Child Psychology 74(2): 107-127.

Schiffer, Stephen (1996) "Contextualist solutions to scepticism", Proceedings of the Aristotelian Society New Series 96: 317-333.

Sennet, Adam (2011) “Ambiguity”, in E. Zalta (ed), Stanford encyclopedia of philosophy (Summer 2011 edition), URL= <http://plato.stanford.edu/archives/sum2011/entries/ambiguity/>. Accessed 15 May 2014.

Sephus, Nashlie, Aaron Lanterman and David Anderson (2015) "Modulation spectral features: in pursuit of invariant representations of music with application to unsupervised source identification", Journal of New Music Research 44(1): 58-70.

Sethares, William and Godfried Toussaint (2015) "Expressive timbre and timing in rhythmic performance: analysis of Steve Reich's Clapping Music", Journal of New Music Research 44(1): 11-24.

Seyfarth, Robert and Dorothy Cheney (1990) "The assessment by vervet monkeys of their own and another species' alarm calls”, Animal Behaviour 40(4): 754-764.

Shanley, Daryl, Rebecca Sear, Ruth Mace and Thomas Kirkwood (2007) "Testing evolutionary theories of menopause", Proceedings of the Royal Society of London B: Biological Sciences 274: 2943-2949.

Sharps, Matthew, Amy Boothby Villegas, Michael Nunes, Terry Barber (2002) "Memory for animal tracks: a possible cognitive artifact of human evolution", The Journal of Psychology 136(5): 469-492.

Sharrock, Justine (2010) Tortured: how our cowardly leaders abused prisoners, American soldiers, and everything we're fighting for (New Jersey: Wiley).

Shaw-Williams, Kim (2014) "The social trackways theory of the evolution of human cognition", Biological Theory 9(1): 16-26.

Shennan, Stephen (2001) "Demography and cultural innovation: a model and its implications for the emergence of modern human culture”, Cambridge Archaeological Journal 11(1): 5-16.

Shiner, Larry (2001) The invention of art: a cultural history (Chicago: University of Chicago Press).

Shipton, Ceri (2013) A million years of hominin sociality and cognition: Acheulean bifaces in the HunsgiBaichbal Valley, India. British Archaeological Reports International Series 2468. (Oxford:

Archaeopress). 
Shipton, Ceri and Mark Nielsen (2015) "Before cumulative culture: the evolutionary origins of overimitation and shared intentionality", Human Nature 26: 331-345.

Silberbauer, George (1981) Hunter and habitat in the central Kalahari desert (Cambridge: Cambridge University Press).

Skidelsky, Edward (2010) “The art instinct”, British Journal of Aesthetics 50(1): 109-112.

Sloboda, John (2005) Exploring the musical mind (Oxford: Oxford University Press).

Slocombe, Katie and Klaus Zuberbühler (2007) "Chimpanzees modify recruitment screams as a function of audience composition", Proceedings of the National Academy of Sciences USA 104: 17228-17233.

Smart, J. J. C. (1959) “Sensations and brain processes”, Philosophical Review 68: 141-156.

Snyder, Jane (1998) "Sappho and other women musicians in Attic vase painting" in A. Buckley (ed), Hearing the past: essays in historical ethnomusicology and the archaeology of sound (Liège: Université de Liège), pp. 165-190.

Soley, Gaye and Erin Hannon (2010) "Infants prefer the musical meter of their own culture: a crosscultural comparison”, Developmental Psychology 46(1): 286-292.

Sorensen, Roy (2009) "Hearing silence: the perception and introspection of absences", in M. Nudds, C. O'Callaghan (eds), Sounds and perceptions: new philosophical essays (New York: Oxford University Press), pp. 126-145.

Spencer, Herbert (1891) "The origin and function of music", in Essays: Scientific, Political, and Speculative, volume II (London: Williams and Norgate), pp. 400-451.

Sperber, Dan (1996) Explaining culture: a naturalistic approach (Oxford: Blackwell).

Stalinsky, Stephanie and E. Glenn Schellenberg (2012) "Music cognition: a developmental perspective", Topics in Cognitive Science 4(4): 485-497.

Standley, Jayne (2000) "The effect of contingent music to increase non-nutritive sucking of premature infants", Pediatric Nursing 26(5): 493-495, 498-499.

Standley, Jayne (2003) "The effect of music-reinforced non-nutritive sucking on feeding rate of premature infants”, Journal of Pediatric Nursing 18: 169-173.

Standley, Jayne (2012) "Music therapy research in the NICU: an updated meta-analysis", The Journal of Neonatal Nursing 31(5): 311-316.

Stanley, Jason (2001) “Hermeneutic fictionalism”, Midwest Studies in Philosophy 25(1): 36-71.

Stecker, Robert (2009) "Methodological questions about the ontology of music", Journal of Aesthetics and Art Criticism 67(4):375-386.

Sterelny, Kim (1990) The representational theory of mind (Oxford: Blackwell).

Sterelny, Kim (2011) "From hominins to humans: how sapiens became behaviourally modern", Philosophical Transactions of the Royal Society B: Biological Sciences 366: 809-822.

Sterelny, Kim (2012a) "Language, gesture, skill: the co-evolutionary foundations of language", Philosophical Transactions of the Royal Society B: Biological Sciences 367: 2141-2151. 
Sterelny, Kim (2012b) The evolved apprentice (Cambridge: MIT Press).

Sterelny, Kim (2014) "Constructing the cooperative niche", in G. Barker, E. Desjardins, T. Pearce (eds), Entagled life: organism and environment in the biological and social sciences (Netherlands: Springer), pp. 261-279.

Sterelny, Kim (2016a) “Deacon's challenge: from calls to words”, Topoi 35(1): 271-282.

Sterelny, Kim (2016b) "Cumulative cultural evolution and the origins of language", Biological Theory 11(3): 173-186.

Sterelny, Kim (2016c) "Cooperation, culture, and conflict", British Journal for the Philosophy of Science 67(1): 31-58.

Sterelny, Kim (2017a) “Language: from how-possibly to how-probably?”, in R. Joyce (ed), Routledge companion to evolution and philosophy (Oxon: Routledge).

Sterelny, Kim (2017b) “Religion re-explained”, Religion, Brain and Behaviour.

Sterelny, Kim and Paul Griffiths (1999) Sex and death: an introduction to philosophy of biology (Chicago: University of Chicago Press).

Stevens, Catherine (2012) "Music perception and cognition: a review of recent cross-cultural research", Topics in Cognitive Science 4(4): 653-667.

Stiner, Mary (2002) "Carnivory, coevolution, and the geographic spread of the genus Homo", Journal of Archaeological Research 10(1): 1-63.

Stiner, Mary (2014) "Finding a common bandwidth: causes of convergence and diversity in Paleolithic beads", Biological Theory 9(1): 51-64.

Stiner, Mary, Natalie Munro and Todd Surovell (2000) "The tortoise and the hare: small-game use, the broad-spectrum revolution, and Paleolithic demography", Current Antropology 41(1): 39-73.

Stone, Ruth (2008) Theory for ethnomusicology (New Jersey: Pearson).

Strawson, P. F. (1959) Individuals (New York: Routledge).

Stubington, Jill (2007) Singing the land: the power of performance in Aboriginal life (New South Wales: Currency House).

Suddendorf, Thomas (2013) The gap: the science of what separates us from other animals (New York: Basic Books).

Sweetser, Eve (1986) “Polysemy vs. abstraction: mutually exclusive or complementary?", Proceedings of the Twelfth Annual Meeting of the Berkeley Linguistics Society, pp. 528-538.

Tabery, James (2014) Beyond versus: the struggle to understand the interaction of nature and nurture (Cambridge: MIT Press).

Temperley, David (2014) “Probabilistic models of melodic interval”, Music Perception 32(1): 85-99.

Tenaza, Richard and Ronald Tinzen (1977) “Evolution of long-distance alarm calls in Kloss's gibbon", Nature 268: 233-235. 
Tesoriero, Michael and Nikki Sue Rickard (2012) "Music-enhanced recall: an effect of mood congruence, emotion arousal or emotion function?”, Musicae Scientiae 16(3): 340-356.

Testart, Alain, Richard Forbis, Brian Hayden, Tim Ingold, Stephen Perlman, David Pokotylo, Peter Rowley-Conwy and David Stuart (1982) "The significance of food storage among hunter-gatherers: residence patterns, population densities, and social inequalities", Current Anthropology 23(5): 523-537.

Thaut, M. H., G. C. McIntosh and R. R. Rice (1997) "Rhythmic facilitation of gait training in hemiparetic stroke rehabilitation”, Journal of Neurological Sciences 151: 207-212.

Thieme, Hartmut (1997) “Lower Palaeolithic hunting spears from Germany”, Nature 385: 807-810.

Thomasson, Amie (2006) “Debates about the ontology of art: what are we doing here?", Philosophy Compass 1(3): 245-255.

Thomasson, Amie (2010) "Ontological innovation in art", Journal of Aesthetics and Art Criticism 68(2): 119-130.

Thompson, William Forde, E. Glenn Schellenberg and Gabriela Husain (2004) "Decoding speech prosody: do music lessons help?", Emotion 4(1): 46-64.

Tillman, Chris (2011) “Musical materialism”, British Journal of Aesthetics 51(1): 13-29.

Titon, Jeff Todd (1996) "The music-culture as a world of music", in J. Titon (ed), Worlds of Music: an introduction to the music of the world's people (New York: Schirmer), pp. 1-32.

Tomasello, Michael (2009) Why we cooperate (Cambridge: MIT Press).

Tomasello, Michael and Malinda Carpenter (2007) "Shared intentionality", Developmental Science 10: 121-125.

Tomasello, Michael, Malinda Carpenter, Josep Call, Tanya Behne and Henrike Moll (2005)

"Understanding and sharing intentions: the origins of cultural cognition", Behavioral and Brain Sciences 28(5): 675-691.

Tomlinson, Gary (2015) A million years of music: the emergence of human modernity (New York: Zone).

Tormey, Alan (1974) “Indeterminacy and identity in art”, Monist 58(2): 203-215.

Trainor, Laurel (2005) “Are there critical periods for musical development?", Developmental Psychobiology 46(3): 262-278.

Trainor, Laurel and Kathleen Corrigall (2010) "Music acquisition and effects of musical experience", in M. R. Jones, R. R. Fay, A. N. Popper (eds), Music Perception (New York: Springer), pp. 89-127.

Trainor, Laurel, Christine Tsang and Vivian Cheung (2002) "Preference for sensory consonance in 2- and 4-month old infants", Music Perception 20(2): 187-194.

Trehub, Sandra (2000) "Human processing predispositions and musical universals", in N. Wallin, B. Merker, S. Brown (eds), Origins of music (Cambridge: MIT Press), pp. 427-448.

Trehub, Sandra (2001) "Musical predispositions in infancy", Annals of the New York Academy of Sciences 930: 1-16.

Trehub, Sandra (2003) "The developmental origins of musicality", Nature Neuroscience 6(7): 669-673. 
Trehub, Sandra, Judith Becker and lain Morley (2015) "Cross-cultural perspectives on music and musicality”, Philosophical Transactions B: Biological Sciences 370(1664), doi. 10.1098/rstb.2014.0096.

Trehub Sandra and Laurel Trainor (1998) "Singing to infants: lullabies and play songs", Advances in infancy research 12: 43-77.

Treitler, Leo (1982) "The early history of music writing in the West", Journal of the American Musicological Society 35(2): 237-279.

Treitler, Leo (1992) “The 'unwritten' and 'written transmission' of medieval chant and the start-up of musical notation”, The Journal of Musicology 10(2): 131-191.

Trestman, Michael (2015) “Clever Hans, Alex the parrot, and Kanzi: what can exceptional animal learning teach us about human cognitive evolution?”, Biological Theory 10(1): 86-99.

Trivedi, Saam (2002) "Against musical works as eternal types", British Journal of Aesthetics 42(1): 73-82.

Trivedi, Saam (2008) “Music and metaphysics", Metaphilosophy 39(1): 124-143.

Trogdon, Kelly and Paisley Livingston (2014) "The complete work", Journal of Aesthetics and Art Criticism 72(3): 225-233.

Tugman, Lindsey (2011) "Oldest cave drawings found in Romanian cave”, CBS News, URL= <http://www.thv11.com/news/article/171246/288/Oldest-cave-drawings-found-in-Romanian-cave/>. Accessed 10 August 2014.

Turner, Jonathan (2000) On the origins of human emotions: a sociological inquiry into the evolution of human affect (Stanford: Stanford University Press).

Tuttle, Russel (2014) Apes and human evolution (Cambridge: Harvard University Press).

Twomey, Terrence (2013) "The cognitive implications of controlled fire use by early humans", Cambridge Archaeological Journal 23(1): 113-128.

Unyk, Anna, Sandra Trehub, Laurel Trainor and E. Glenn Schellenberg (1992) "Lullabies and simplicity: a cross-cultural perspective", Psychology of Music 20: 15-28.

Van Inwagen, Peter (1990) Material beings (Ithaca: Cornell University Press).

Varèse, Edgard (1966) “The liberation of sound”, Perspectives of New Music 5(1): 11-19.

Vaughan, C. David (2001) "A million years of style and function: regional and temporal variation in Acheulean handaxes", in T. D. Hurt and G. F. M. Rakita (eds), Style and function: conceptual issues in evolutionary archaeology (Connecticut: Bergin \& Garvey), pp. 141-163.

Verspoor, Marjolijn and Wander Lowie (2003) "Making sense of polysemous words", Language Learning 53(3): 547-586.

Villmoare, Brian, William Kimbel, Chalachew Seyoum, Christopher Campisano, Erin DiMaggio, John Rowan, David Braun, J. Ramón Arrowsmith and Kaye Reed (2015) "Early Homo at 2.8 ma from LediGeraru, Afar, Ethiopia", Science 347(6228): 1352-1355.

Wadley, Lyn (2010) "Were snares used in the Middle Stone Age and does it matter? A review and case study from Sibidu, South Africa", Journal of Human Evolution 58(2): 179-192. 
Wagner, Günter (2010) “The measurement theory of fitness”, Evolution 64(5): 1358-1376.

Wallin, Nils, Björn Merker and Steve Brown (eds) (2000) Origins of music (Cambridge: MIT Press).

Walton, Kendall (1970) “Categories of art”, Philosophical Review 79(3): 334-367.

Walton, Kendall (1988) "The presentation and portrayal of sound patterns", in J. Dancy, J. M. E. Moravcsik, C. C. W. Taylor (eds), Human agency: language, duty, and value (Stanford: Stanford University Press), pp. 237-257.

Walton, Kendall (1990) Mimesis as make-believe (Cambridge: Harvard University Press).

Walton, Kendall (2005) "Metaphor and prop oriented make-believe", in M. E. Kalderon (ed), Fictionalism in metaphysics (New York: Oxford University Press), pp. 65-87.

Warner, Daniel (ed) (2006) Audio culture: readings in modern music (New York: Continuum).

Warnock, G. J. (1982) Berkeley (Oxford: Blackwell).

Waterman, Richard (1956) "Music in Australian aboriginal culture-some sociological and psychological implications", in E. T. Gaston (ed), Music therapy 1955 (Kansas: Proceedings of the National Association for Music Therapy), pp. 40-49.

West, M. L. (1992) Ancient Greek music (Oxford: Clarendon Press).

Whiten, Andrew (2015) "Experimental studies illuminate the cultural transmission of percussive technologies in Homo and Pan", Philosophical Transactions of the Royal Society: Biological Sciences, doi. 10.1098/rstb.2014.0359.

Wiessner, Polly (2014) "Embers of society: firelight talk among the Ju/'hoansi Bushmen", Proceedings of the National Academy of Sciences USA 111: 14027-14035.

Williamson, Victoria (2014) You are the music: how music reveals what it means to be human (London: Icon).

Winkler, István, Gábor Háden, Olivia Ladinig, István Sziller and Henkjan Honing (2009) “Newborn infants detect the beat in music", Proceedings of the National Academy of Sciences USA 106: 1479-1489.

WoldeGabriel, Giday, Grant Heiken, Tim White, Berhane Asfaw, William Hart and Paul Renne (2000) "Volcanism, tectonism, sedimentation, and the paleoanthropological record in the Ethiopian Rift System", in F. W. McCoy and G. Heiken (eds), Volcanic Hazards and Disasters in Human Antiquity (Boulder, Colorado: Geological Society of America Special Paper 345), pp. 83-99.

WoldeGabriel, Giday, Yohannes Haile-Selassie, Paul Renne, William Hart, Stanley Ambrose, Berhane Asfaw, Grant Heiken and Tim White (2001) "Geology and paleontology of the Late Miocene Middle Awash valley, Afar rift, Ethiopia”, Nature 412: 175-178.

Wolterstorff, Nicholas (1980) Works and worlds of art (Oxford: Clarendon Press).

Wrangham, Richard (2009) Catching fire: how cooking made us human (London: Profile).

Wrangham, Richard and Rachel Carmody (2010) "Human adaptation to the control of fire", Evolutionary Anthropology 19(5): 187-199. 
Wrangham, Richard and NancyLou Conklin-Brittain (2003) "Cooking as a biological trait", Comparative Biochemistry and Physiology Part A: Molecular and Integrative Physiology 136(1): 35-46.

Wray, Alison (2002) Formulaic language and the lexicon (Cambridge: Cambridge University Press).

Wray, Alison (2008) Formulaic language: pushing the boundaries (Oxford: Oxford University Press).

Wu Zhao (1979) "Rare find of ancient instruments", China Reconstructs 28(5): 28-31.

Wu Zhao (1980) "Guqin - the Chinese zither", Chinese Literature 12(12): 110-113.

Wylie, Alison (1999) "Rethinking unity as a 'working hypothesis' for philosophy: how archaeologists exploit the disunities of science", Perspectives On Science 7(3): 293-317.

Yablo, Stephen (2001) "Go figure: a path through fictionalism", Midwest Studies in Philosophy 25(1): 72-102.

Young, James (2011) “The ontology of musical works: a philosophical pseudo-problem”, Frontiers of Philosophy in China 6(2): 284-297.

Zangwill, Nick (2014) "Re-centring musicology and the philosophy of music", Journal of Aesthetics and Phenomenology 1: 231-240.

Zarco, Wilbert, Hugo Merchant, Luis Prado and Juan Carlos Mendez (2009) "Subsecond timing in primates: comparison of interval production between human subjects and rhesus monkeys", Journal of Neurophysiology 102: 3191-3202.

Zilhão, João (2007) "The emergence of ornaments and art: an archaeological perspective on the origins of 'behavioral modernity”, Journal of Archaeological Research 15(1): 1-54.

Zuberbühler, Klaus, David Jenny and Redouan Bshary (1999) "The predator deterrence function of primate alarm calls", Ethology 105(6): 477-490.

Zubrow, Ezra, Ian Cross and Frank Cowan (2001) "Musical behaviour and the archaeology of the mind", Archaeologia Polona 39: 111-126.

Zwicky, Arnold and Jerrold Sadock (1975) "Ambiguity tests and how to fail them", Syntax and Semantics 4(1): 1-36. 